\title{
Attività sismica in Italia dal 1953 al 1957
}

\author{
M. DE PANFILIS
}

L'attività sismica verificatasi in Italia durante il quinquennio 19531957 , se confrontata con quella che si è avuta nel passato in periodi di tempo di uguale durata, non può dirsi. per quanto riguarda la frequenza, molto notevole. In tale periodo, le scosse sismiche di cui abbiamo potuto avere sicura notizia, comprese quelle di leggera e leggerissima intensità e le repliche dei sismi più importanti, hanno superato di poco il numero di 400 . Invece tale attività si deve considerare piuttosto elevata nei riguardi dell'intensità, poichè 160 delle scosse predette hanno superato il IV grado della Scala Mercalli. Due di esse, anzi, l'una avvenuta nel Gargano nel febbraio 1955 e l'altra nei Monti Volsini, a nord del Lago di Bolsena, nel dicembre 1957, raggiunsero nella zona epicentrale un'intensità quasi rovinosa, fortunatamente senza vittime, ma con danni ingenti ai fabbricati.

Assai diversamente, poi, si è distribuita l'attività sismica nei singoli anni del quinquennio. La minima si è avuta nel 1954 con un totale di 52 scosse e la massima nel 1957 con un totale di 123 scosse.

Secondo l'intensità massima raggiunta all'epicentro, espressa in gradi della Scala Mercalli, i sismi avvenuti nel periodo in esame possono essere così ripartiti:

\begin{tabular}{|c|c|c|c|c|c|c|c|c|c|c|}
\hline Cirado & II & III & IV & IV-V & V & V-VI & VI & VI-VII & VII & VII-VIII \\
\hline $\begin{array}{l}\text { Numero } \\
\text { di scosse }\end{array}$ & 40 & 110 & 105 & 49 & 61 & 10 & 23 & 6 & 9 & 2 \\
\hline
\end{tabular}

A queste scosse, che sono quelle ben determinate sia rispetto al luogo ed al tempo che all'intensità, se ne debbono aggiungere numerose altre, di cui però si sono avute solo vaghe ed imprecise notizie, tutte di lieve entità e principalmente repliche dei terremoti più importanti.

Riguardo poi alla distribuzione geografica delle scosse di grado uguale e superiore al V, risulta che, di esse, 4 hanno avuto il loro epi- 
centro in Piemonte, una nel Trentino, una nel Veneto, 7 nel Friuli, 3 in Liguria, 12 in Emilia, 10 in Toscana, 9 in Umbria, 5 nelle Marche, 5 nel Lazio, 7 negli Abruzzi e Molise, 3 in Campania, 14 in Puglia, 6 in Lucania, 8 in Calabria, 13 in Sicilia e 3 nelle Isole Eolie.

Il maggior numero di manifestazioni sismiche si è avuto pertanto in Puglia. I relativi epicentri si addensano quasi tutti nella regione montuosa del Gargano, tristamente nota per la sua elevata sismicità. Una di esse, quella del 9 febbraio 1955, fu d'intensità quasi rovinosa e causò danni gravi a molte case di Monte S. Angelo, la graziosa cittadina che si eleva su uno sperone meridionale del Gargano. La Sardegna ha confermato la sua relativa asismicità, non essendovisi verificata alcuna scossa dal 1953 al 1957.

Nel periodo in esame, le manifestazioni sismiche più importanti, per l'elevata intensità e per i conseguenti effetti sulle costruzioni nelle zone epicentrali, sono rappresentate, oltre che dall'anzidetto terremoto garganico del febbraio 1955, da due periodi sismici svoltisi nell'anno 1957 . II primo ebbe Iuogo nell'Appennino emiliano e colpi specialmente il paese di Santa Sofia; il secondo ebbe il suo epicentro nei Monti Volsini, a nord del Lago di Bolsena, e arrecò sensibili danni in numerosi paesi di quella regione, specialmente negli abitati di Castel Giorgio e di Castel Viscardo.

Con il presente lavoro, in cui vengono passati in rassegna i terremoti di grado $\mathrm{V}$ e quelli di grado superiore, abbiamo voluto illustrare l'attività sismica manifestatasi nelle singole regioni d'Italia dal 1953 al 1957. Eccetto per quei casi in cui le notizie a nostra disposizione erano insufficienti, abbiamo completato la descrizione macrosismica di ogni terremoto con il disegno delle relative isosiste. La carta generale degli epicentri, che accompagna il testo, dà una visione sintetica della distribuzione geografica dei terremoti predetti e, a facilitarne la consultazione, abbiamo scritto a fianco di ciascun epicentro il numero con il quale il sisma relativo è contrassegnato nelle pagine seguenti. Come appare dalla carta, alcuni epicentri sono situati nella catena alpina e, precisamente, nelle Alpi Marittime, nelle Alpi Cozie e, in maggior numero, nelle Alpi Carniche. La maggior parte, però, sono distribuiti lungo tutto l'areo appenninico, specialmente nell'Appennino tosco-emiliano, in quello abruzzese e in quello lucano. Esternamente alla catena degli Appennini è soprattutto da rilevare l'addensamento di epicentri nella zona montuosa del Gargano, che, nel periodo di tempo esaminato, fu, fra tutte le zone d'Italia, quella in cui l'attività sismica si manifestò con maggiore frequenza. 


\section{Anno 1953}

1. 25 febbraio

Filogaso (Catanzaro)

Grado VI

Il 25 febbraio 1953 , alle $01^{\text {h }} 07^{\mathrm{m}}$ circa, fu avvertita, su quasi tutto il versante tirrenico della Calabria, in molte località delle province di Messina e di Catania, nonchè in alcune ristrette zone delle Puglie, una scossa di terremoto che, pur non avendo raggiunto nella zona epicentrale una intensità molto elevata, risultò di propagazione macrosismica assai rilevante (fig. 1 ).

Nel comune di Filogaso, in provincia di Catanzaro, ove la scossa fu sentita con l'intensità di VI grado, si verificarono fenditure in edifici di difettosa costruzione e crolli di alcuni vecchi muri. Con intensità un pò minore il movimento tellurico fu avvertito nel vicino comune di S. Onofrio.

L'epicentro fu individuato dall'I.N.G. nel punto di coordinate $38^{\circ} 42^{\prime} \mathrm{N}$ e $16^{\circ} 06^{\prime} \mathrm{E}$.

Come mostra la fig. 1, la zona megasismica è di estensione relativamente piccola ma la isosista di grado $\mathrm{V}$, avente la forma di un'ellisse molto allungata con l'asse maggiore orientato da SSW a NNE, abbraccia un territorio assai vasto estendentesi principalmente lungo la costa tirrenica all'incirca da Cleto (Cosenza) a Messina. La isosista di grado IV, pure di forma ellittica, si estende sulla costa tirrenica da Diamante (Cosenza) fin oltre Milazzo (Messina) e racchiude nel suo interno anche l'Isola di Stromboli.

Sul versante jonico della Calabria il movimento tellurico non fu quasi affatto avvertito. Lo fu invece, a distanza molto grande dalla zona epicentrale, in alcune località delle Puglie: di IV grado a Taranto, a Mola di Bari (Bari) e a Trepuzzi (Lecce), di III grado a Galatina (Lecce).

La estesa area macrosismica e Ia irregolarità di propagazione del moto tellurico indicano una notevole profondità ipocentrale. L'ora origine del terremoto, desunta dalle registrazioni ottenute negli Osservatorî di Messina, Taranto, Roma, Firenze e Saló è $01^{\text {h }} 07^{\mathrm{m}} 46^{\mathrm{s}}$.

Una leggera scossa, probabile replica della precedente, fu avvertita nella zona del Monte Poro alle $10^{\mathrm{h}} 15^{\mathrm{m}}$ circa del 27 febbraio.

\section{26 febbraio Monteleone di Spoleto (Perugia) Grado $\mathrm{V}$}

Di questa scossa, che fu di carattere strettamente locale, l'epicentro venne individuato dall'I.N.G. nel punto di coordinate $42^{\circ} 38^{\prime} \mathrm{N} \mathrm{e} 12^{\circ} 55^{\prime} \mathrm{E}$. 


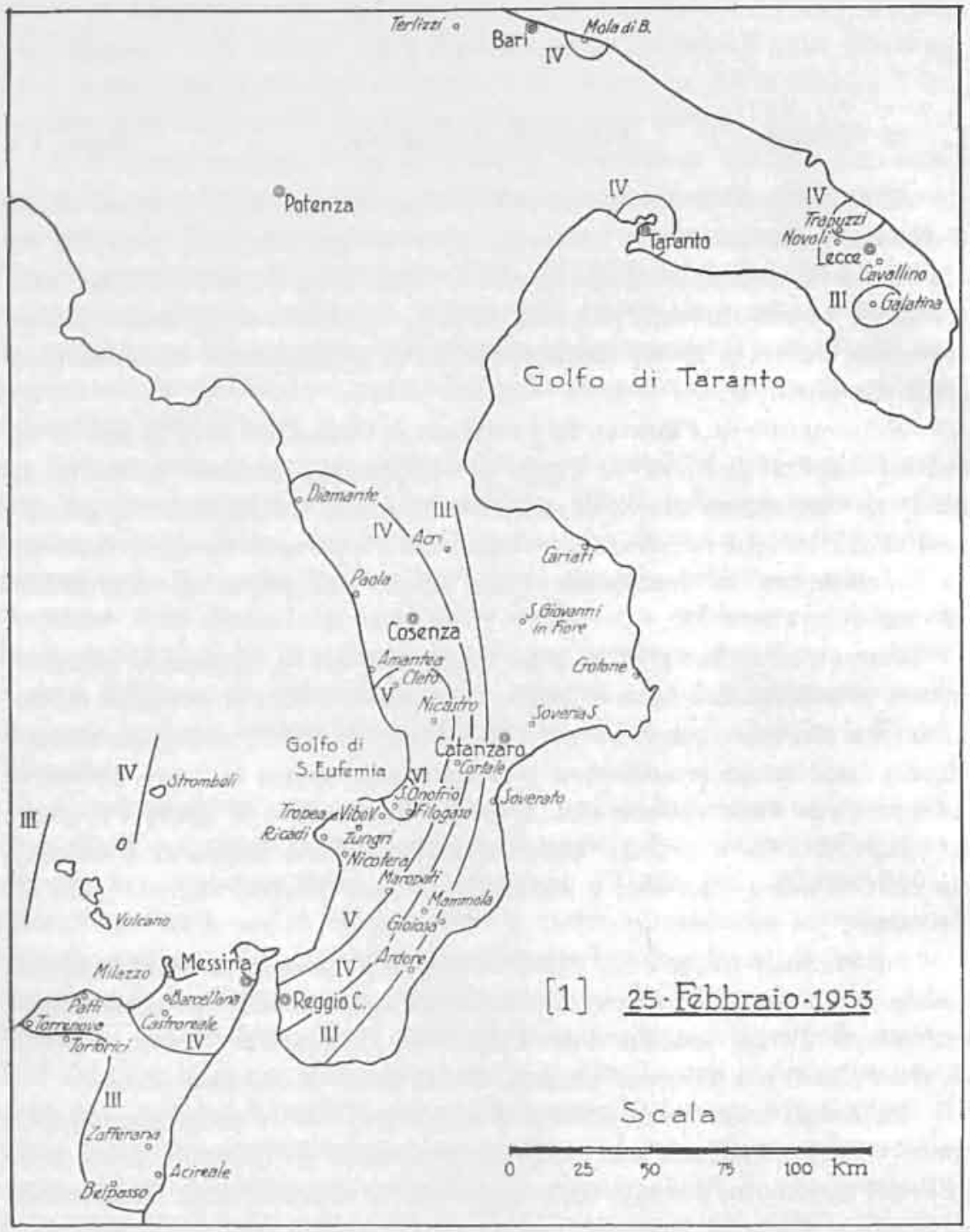

Fig. 1

Esso cade a ENE di Terni nella zona compresa fra Monteleone di Spoleto, Ferentillo (Terni) e Leonessa (Rieti). L'ora origine determinata in base alle registrazioni di Roma, Rocea di Papa, Siena e Firenze è $08^{\mathrm{h}} 34^{\mathrm{m}} 56^{\mathrm{s}}$. 
Per quanto d'intensità inferiore al V grado Mercalli, stimiamo opportuno segnalare, essendosi verificate in una zona generalmente ritenuta asismica, due scosse originatesi nel sottosuolo di Roma il 4 marzo 1953.

La storia sismica ci ha tramandato notizie di terremoti numerosi e violenti sentiti a Roma nell'antichità e per i quali gli edifici dell'Urbe rimasero gravemente danneggiati. Ma, data la vicinanza di attivissimi centri sismici, quali quelli dei Colli Laziali e degli Abruzzi, è difficile stabilire quale sia stato il punto d'origine di quegli antichi movimenti tellurici. $\mathrm{E}$ infatti accaduto anche in tempi recenti che terremoti irradiati dai centri sismici suddetti sono risultati fortissimi in Roma. Per esempio il grande sisma di Avezzano del 13 gennaio 1915 fu sentito a Roma di VII grado, causando, fra altri danni, la caduta di due sfere di pietra dall'alto della Chiesa di S. Ignazio e della statua di S. Paolo dal sommo della facciata della Basilica di S. Giovanni.

I terremoti dei Colli Laziali invero, generalmente assai localizzati data la loro natura prevalentemente vulcanica, di rado si sono propagati fino a Roma con intensità tale da provocare lesioni alle costruzioni. Però il terremoto del 19 luglio 1899, che nella zona epicentrale, comprendente Frascati, Marino e Grottaferrata, raggiunse il grado VIII, si propagò fortemente anche a Roma ove la scossa fu sentita di VI grado.

In generale, sulla sola base dei terremoti avvertiti a Roma dall'antichità fino a quasi tutto il secolo $\mathrm{XIX}^{\circ}$, ben poco potrebbe dirsi con sicurezza circa la sismicità locale della zona urbana e di quella immediatamente contigua (*). Ma un terremoto avvenuto appunto sul finire del secolo scorso (il $1^{\circ}$ novembre 1895 ), un altro verificatosi nel primo decennio di questo secolo (il 31 agosto 1909) e le due scosse del 4 marzo 1953 pongono fuori dubbio l'esistenza neI sottosuolo di Roma di alcuni centri sismici, sia pure di assai modesta attività.

Del terremoto del $1^{\circ}$ novembre 1895 il De Rossi pose l'epicentro a sud di Roma, nei pressi della Cechignola, fra le Vie Ostiense ed Ardeatina (fig. $2 ;$ n. 1), località allora pressochè deserta ma oggi raggiunta $\mathrm{e}$ superata dalle costruzioni nel grande sviluppo assunto dalla Città in dire-

(*) M. Baratta, nel suo volume sui terremoti d'Italia, ricorda un sisma avvenuto a Roma il 22 marzo 1812 e il cui epicentro può ritenersi essere stato nella zona urbana o almeno nelle sue immediate vicinanze $\left(^{1}\right)$. 
zione di Ostia. Secondo il Baratta, invece, l'epicentro sarebbe stato in mare, vicino a quella parte della costa ove si estendono gli edifici del Lido di Roma. Ma forse, valutate tutte le circostanze, si è nel vero ponendo l'epicentro, come pensò il Martinelli, a SSW di Roma fra la Città

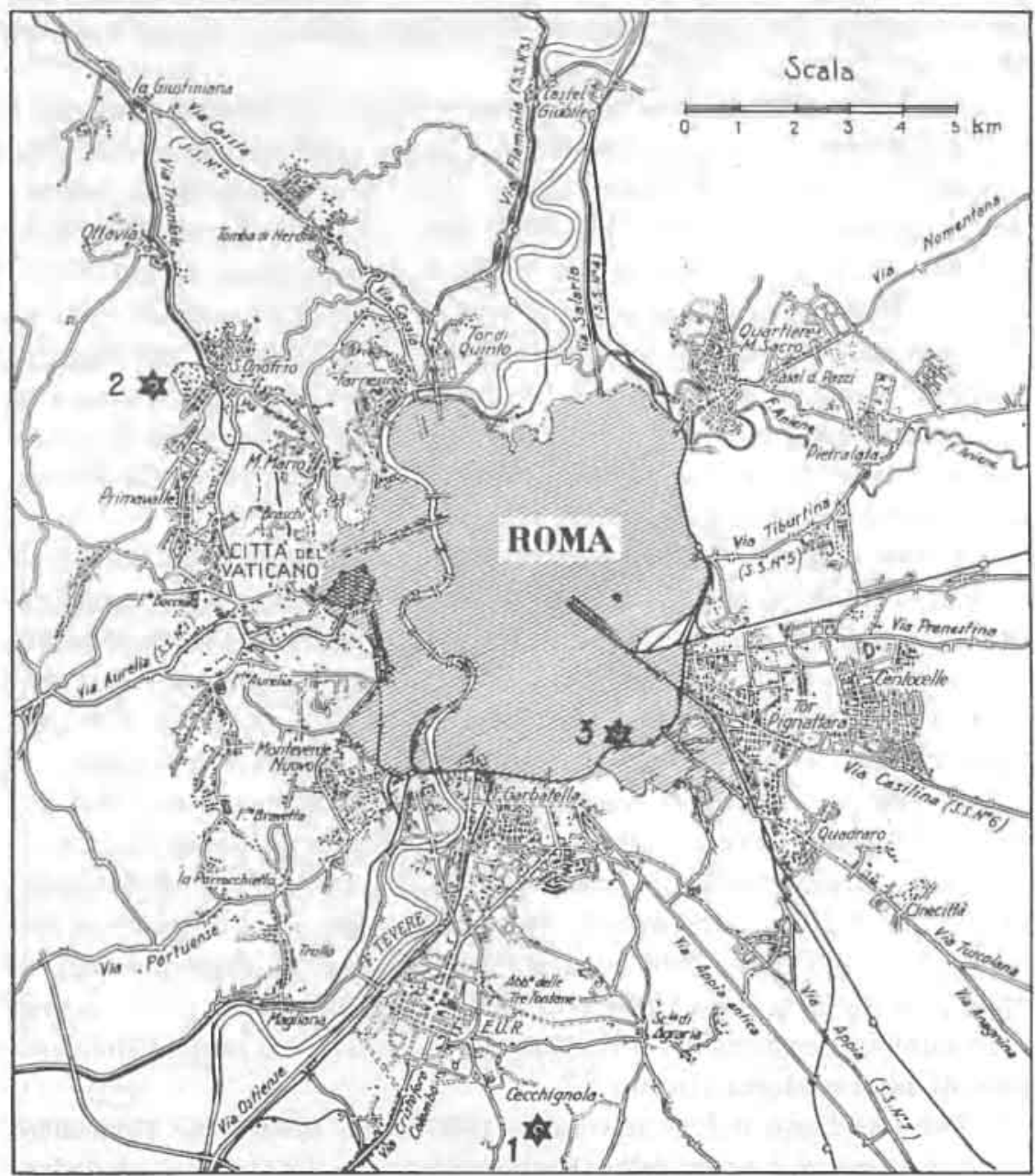

Fig. 2

ed il mare. A Roma, a Fiumicino e ad Ostia Antica la scossa, che in ogni caso può dirsi di carattere locale rispetto a Roma, raggiunse il VI grado e produsse lesioni che, in edifici vecchi e mal costruiti, furono anche di una certa gravità. 
Il terremoto del 31 agosto 1909, giudicato d'intensità fra il $\mathrm{V}$ e il VT grado, produsse danni lievi in alcuni edifiei e lesioni gravi in qualche corsia dell'Ospedale di S. Spirito. ì da tener presente però che le condizioni statiche del vetusto edificio erano state in quell'anno turbate da alcuni lavori che si stavano eseguendo a poca distanza da esso per la costruzione dei muraglioni del Tevere. I'epicentro del sisma fu individuato in un punto a circa sette chilometri dal centro di Roma in direzione nord-ovest, cioè nei pressi di S. Onofrio (fig. $2 ; \mathrm{n}$. 2), localitè anche questa. oggi raggiunta dallo sviluppo edilizio e densamente popolata.

Delle due scosse del 4 marzo 1953 , la prima avvenne alle $11^{\mathrm{b}} 48 \mathrm{~m} 44^{\mathrm{s}}$ e la seconda pochi minuti dopo, cioè alle $11^{\mathrm{b}} 56^{\mathrm{m}} 29^{\mathrm{s}}$. I sa prima, di IV grado, fu avvertita in gran parte della città, specialmente nei quartieri Appio, Tiburtino, S. Giovanni e Torpignattara; là seconda, un po' più intensa della prima (fra il IV e il V grado), fu sentita in tutta la città, soprattutto nei quartieri meridionali, e suscitò un certo allarme nella popolazione. Ambedue furono registrate, oltre che a Roma, anche a Rocea di Papa. Dalle registrazioni fu possibile dedurre l'epicentro che venne localizzato dall'I.N.G. nel punto di coordinate $41^{\circ} 51^{\prime} 50^{\prime \prime} \mathrm{N}$ e $122^{\circ} 30^{\prime} 43^{\prime \prime} \mathrm{E}$. Tale punto, distante cirra tre chilometri e mezzo dalla Città Unversitaria, ove risiede l'Osservatorio sismieo di Roma, è situato nella zona del Quartiere Appio (fig. 2; n. 3), che è uno di quelli in cui la scossa fu maggiormente sentita. Le notizic subito raccolte mostrarono che la propagazione macrosismica era stata limitatissima, sicchè assai piccola dovette essere la profondità ipocentrale.

Le manifestazioni sismiche romane di cui brevemente si c̀ detto, più che dalla loro intensità, traggono importanza dal fatto che sono le poche che ai permettano, fino ad oggi, di asserire che anche la zona di Roma è una zona dotata di una sua propria sismicità indipendentemente da quella che le proviene dalla vicinanza agli attivi centri sismici dei Colli Laziali, degli Abruzzi, del Viterbese e della Sabina.

3. 21 maggio

Vizzini (Catania)

Grado $Y$

All'epicentro di questo sisma, in base alle registrazioni ottenute negli Osservatorî di Messina e di Reggio Calabria, sono state assegnate le coordinate $37^{\circ} 15^{\prime} \mathrm{N}$ e $14^{\circ} 49^{\prime}$ E. Tale punto si trova nell'angolo sudorientale della Sicilia fra Vizzini e Militello, cioè in quella parte dell'Isola tante volte funestata nel passato da violenti terremoti. 
Assai scarse sono le notizie macrosismiche a nostra disposizione su questo modesto movimento tellurico. Solo sappiamo che esso fu sentito con forte intensità (V grado) a Vizzini e con intensità mediocre (IV grado) a Caltagirone e a Scordia (Catania). Verso nord esso fu sensibile all'uomo fin poco oltre Ramacca.

L'ora origine, dedotta dalle suddette registrazioni di Messina e di Reggio, è di $01^{\mathrm{h}} 36^{\mathrm{m}} 03^{\mathrm{s}}$.

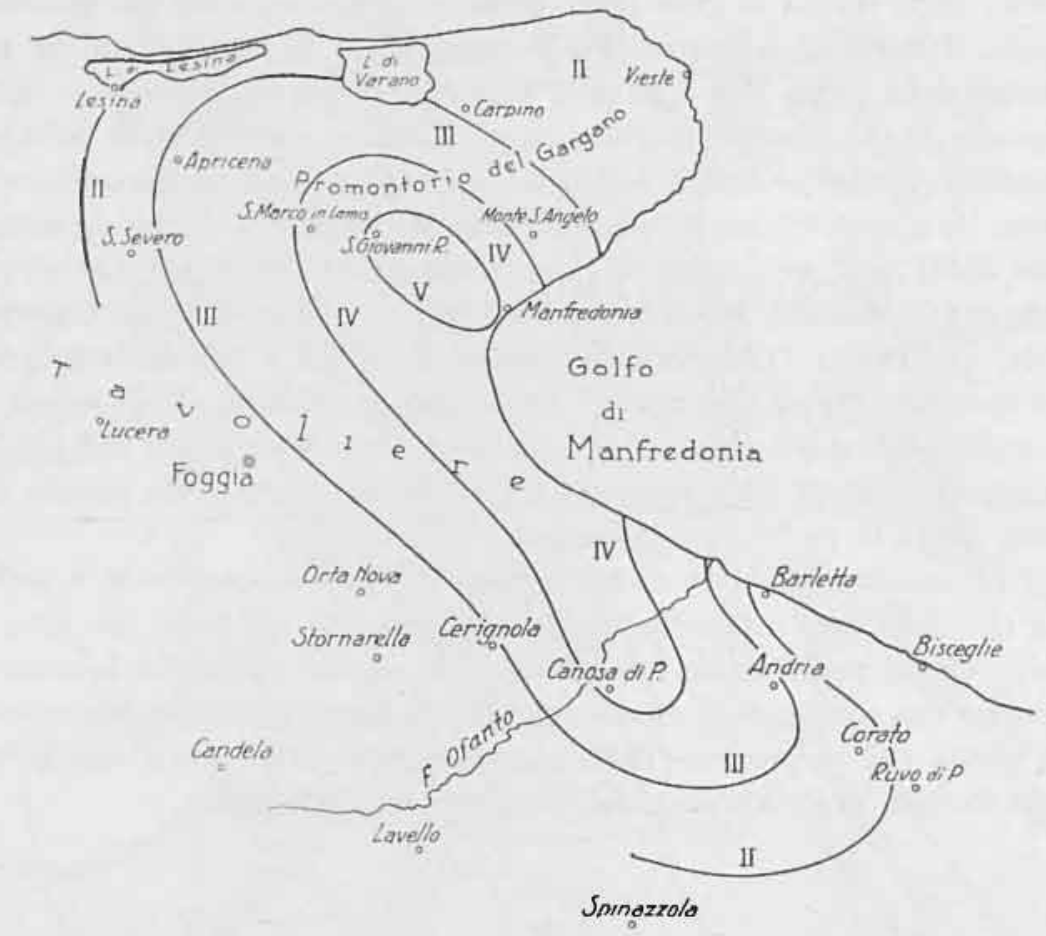

[4] 19.Luglio.1953

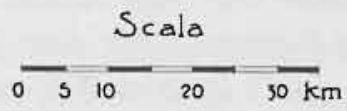

Fig. 3 
4. 19 luglio

S. Giovanni Rotondo (Foggia)

Grado V

Pur non avendo raggiunto nella zona epicentrale un'intensità molto elevata, questo sisma ebbe una notevole propagazione macrosismica. Fu avvertito infatti in tutto il Promontorio garganico e, verso SE, attraverso il Tavoliere di Puglia, fin oltre il fiume Ofanto, a Canosa di Puglia Andria, Corato (fig. 3).

L'epicentro fu probabilmente fra S. Giovanni Rotondo e Manfredonia. Nella prima di queste località la scossa fu sentita con l'intensità del V grado e provocò il crollo di alcuni muri vecchi e già fatiscenti. A Manfredonia l'intensità fu un po' minore (IV-V grado), ma assai vivo fu anche in questa località l'allarme della popolazione.

La scossa fu registrata in diversi Osservatorî italiani e dalle registrazioni risultò come ora origine $19^{\mathrm{h}} 46^{\mathrm{m}} 46^{\mathrm{s}}$.

5. 25. luglio

Arta (Udine)

Grado V

La figura 4 mostra la propagazione di questa scossa il cui epicentro, in base alle registrazioni ottenute a Padova, Salò, Bologna, Siena, Pavia e Roma, è stato ubicato dall'I.N.G. nel punto di coordinate $46^{\circ} 30^{\prime} \mathrm{N}$

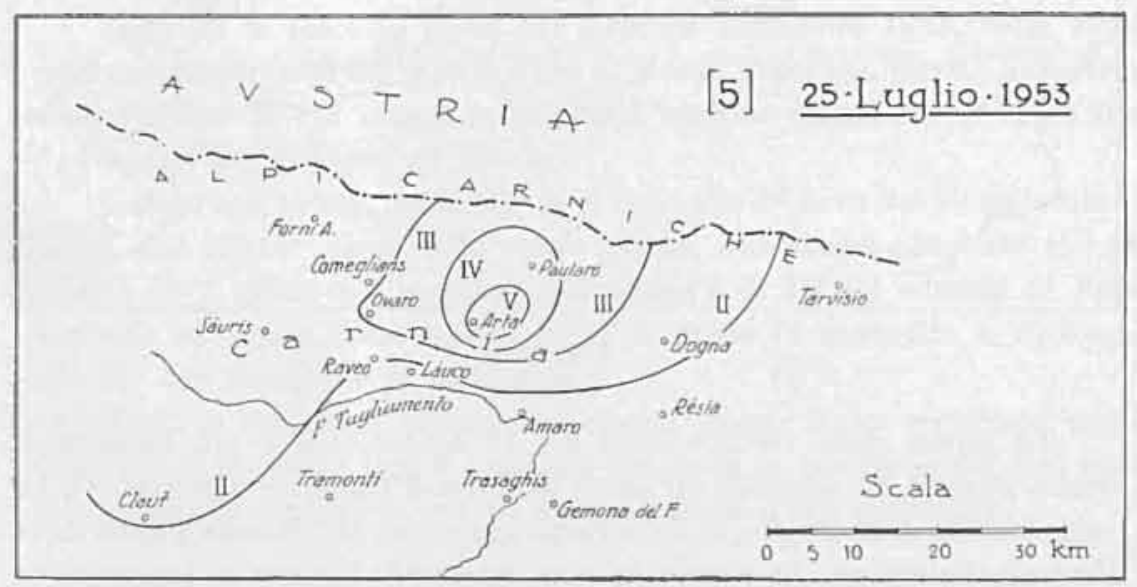

Fig. 4

e $13^{\circ} 06^{\prime}$ E e cioè nella Carnia, fra Arta, ove la scossa fu avvertita fortemente, e Paularo. L'intensità del movimento sismico decrebbe assai rapidamente verso sud, sicchè nei comuni di Lauco ed Amaro, poco distanti da Arta, la scossa non venne affatto avvertita.

L'ora origine del sisma fu $02^{\mathrm{h}} 34^{\mathrm{m}} 50^{\mathrm{s}}$. 
6. 12 agosto

Il 9 agosto 1953 , alle $08^{\mathrm{h}} 41^{\mathrm{m}} 06^{\mathrm{s}}$, con una scossa disastrosa, s'iniziava nelle Isole Eolie un periodo sismico eccezionale sia per la sua durata che per la frequenza e l'intensità delle scosse. Per tutto il mese di agosto le repliche si susseguirono, a brevi intervalli di tempo, con una frequenza impressionante, portando la desolazione nelle Isole joniche di Cefalonia, Itaca e Zante.

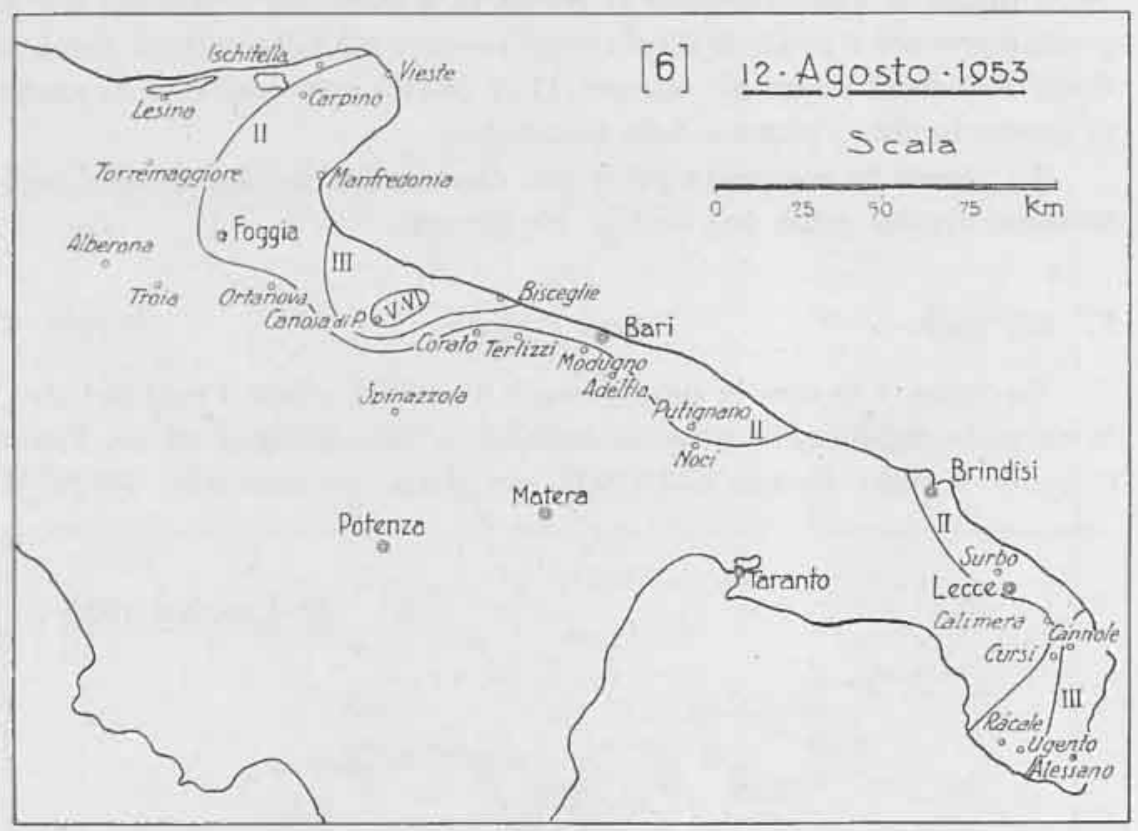

Fig, 5

La scossa delle $10^{\mathrm{h}} 24^{\mathrm{m}}$ circa del 12 agosto, ancor più violenta di quella iniziale, fu sensibile all'uomo anche nell'Italia meridionale (*). In varie località delle Puglie, dalla regione garganica all'estrema punta della Penicola Salentina, fu avvertita con intensità leggera o leggerissima (fig. 5). Ma un rinforzo notevole d'intensità, sì da far pensare addirittura ad una scossa originata da un centro locale, si ebbe nella zona di Canosa

(*) Questo terremoto è stato studiato da D. Di Filippo e da L. Marcelli, i quali, per le coordinate epicentrali e per il tempo origine, determinarono i valori:

$$
\varphi=38^{\circ} 10^{\prime} 26^{\prime \prime}, 8 ; \quad \lambda=20^{\circ} 43^{\prime} 12^{\prime \prime} ; \quad t_{0}=10^{\mathrm{h}} 23^{\mathrm{m}} 50^{\mathrm{s}}\left({ }^{2}\right) .
$$


di Puglia. Ivi infatti fu avvertito un movimento tellurico abbastanza forte, valutato di V-VI grado Mercalli, il quale produsse anche leggiere lesioni in alcune case e la caduta di qualche comignolo.

\section{23 agosto Correggio - Novellara (Reggio E.) Grado V}

Di questo sisma non si sono avute registrazioni negli Osservatori dell'I.N.G. In base alle scarse notizie macrosismiche che di esso si sono potute avere, riteniamo che l'epicentro possa essere collocato nella zona compresa fra Corregyio e Novellara, zona in cui, nel secolo scorso, si ebbero notevoli manifestazioni di attività sismica.

A Correggio la scossa fu valutata di $\mathrm{V}$ grado e a Novellara di IV. Il movimento tellurico ebbe una propagazione alquanto limitata: esso decrebbe molto rapidamente con la distanza, eccetto in direzione sud, essendo stato leggermente percepito a Castellarano, distante una trentina di chilometri da Correggio.

Il sisma si verifico alle $06^{\mathrm{h}} 26^{\mathrm{m}}$ eirca.

8. 20 settembre Spilinga (Catanzaro) Grado V

Durante la seconda metà del mese di settembre 1953, nella zona calabra estendentesi dal Monte Poro al Monte Pecoraro, furono avvertite alcune scosse la cui maggiore intensità sembra essersi manifestata fra Spilinga, Vibo Valentia ed Arena.

Una prima scossa, molto lieve, si ebbe alle $5^{\mathrm{h}}$ circa del 20 settembre. Segui, alle $21^{\mathrm{h}} 15^{\mathrm{m}}$ eirca dello stesso giorno, una scossa più forte che fu sentita di V grado nel comune di Spilinga e di IV nei comuni di Vibo Valentia ed Arena. Una replica di IV-V grado fu avvertita a Spilinga alle $21^{\mathrm{h}} 45^{\mathrm{m}}$ circa del 24 settembre.

Nessuno dei sismi predetti ci risulta essere stato registrato negli Osservatori sismici e non è possibile precisare la posizione del loro epicentro data la scarsità di notizie macrosismiche a noi pervenute.

9. 5 ottobre Cancellara (Potenza) Grado V

La figura 6 mostra le isosiste di questo terremoto tracciato sulla base delle notizie macrosismiche a nostra disposizione.

La scossa fu sentita fortemente a Cancellara, leggermente a Potenza e nei comuni di Venosa, Oppido Lucano, Genzano di Lucania (tutti della prov. di Potenza). 


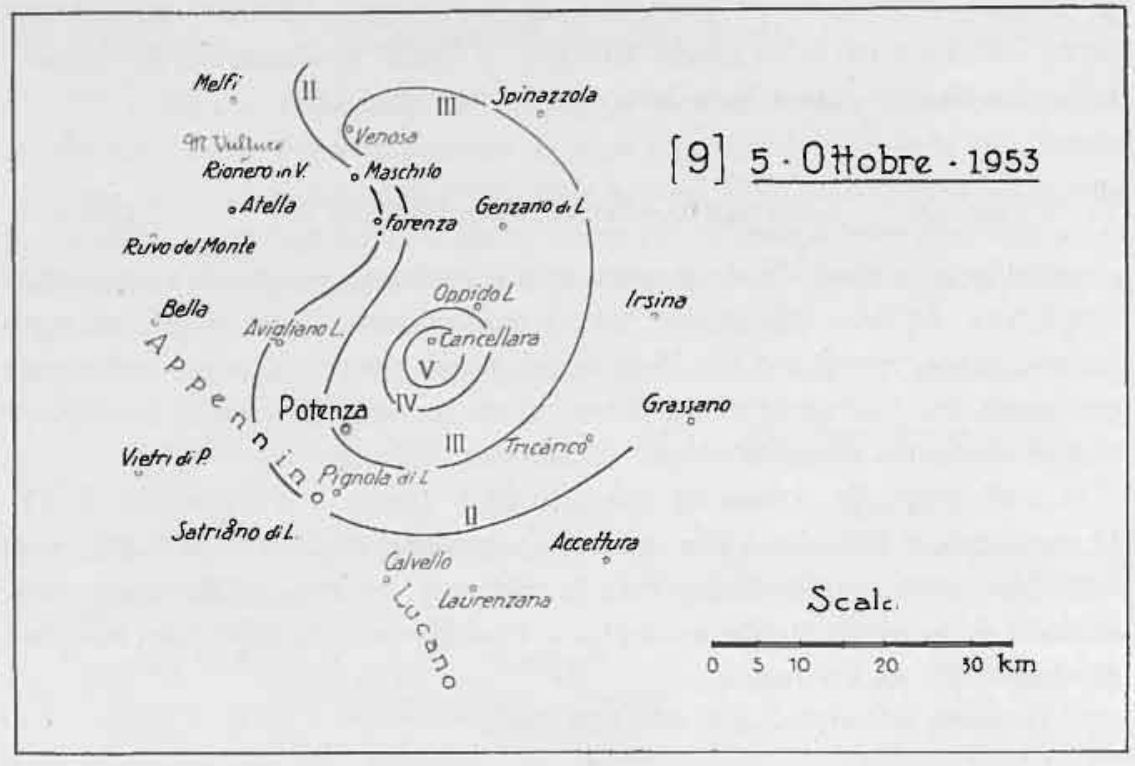

Fig. 6

In base alle registrazioni ottenute negli Osservatorî di Taranto, Messina e Roma, l'epicentro è stato determinato dall'I.N.G. nel punto di coordinate $40^{\circ} 42^{\prime} \mathrm{N}$ e $15^{\circ} 57^{\prime}$ E. Ora origine: $01^{\mathrm{h}} 08^{\mathrm{m}} 19^{\mathrm{s}}$.

10. 9 ottobre

Asciano (Siena)

Grado V

Questa scossa, avvenuta a circa $16^{\mathrm{h}} 21^{\mathrm{m}}$ del 9 ottobre, conferma l'esistenza nei pressi di Asciano, a SE di Siena, di un centro sismico già rivelato nel passato da manifestazioni numerose, anche se non molto intense.

La figura 7 rappresenta la propagazione del moto sismico che, sentito di grado $\mathrm{V}$ ad Asciano, decrebbe rapidamente verso SE, cosi da non essere affatto avvertito a Trequanda, distante da Asciano appena una decina di chilometri in linea d'aria. A Buonconvento e a Rapolano Terme la scossa fu sentita con mediocre intensità; con intensità leggera a Sovicille, Castelnuovo Berardenga, ecc.

In base alle registrazioni ottenute negli Osservatorî di Siena, Firenze e Roma, sono state assegnate all'epicentro le coordinate $43^{\circ} 17^{\prime} \mathrm{N}$ $11^{\circ} 36^{\prime}$ E. Ora origine: $16^{\mathrm{h}} 20^{\mathrm{m}} 57^{\mathrm{s}}$. 


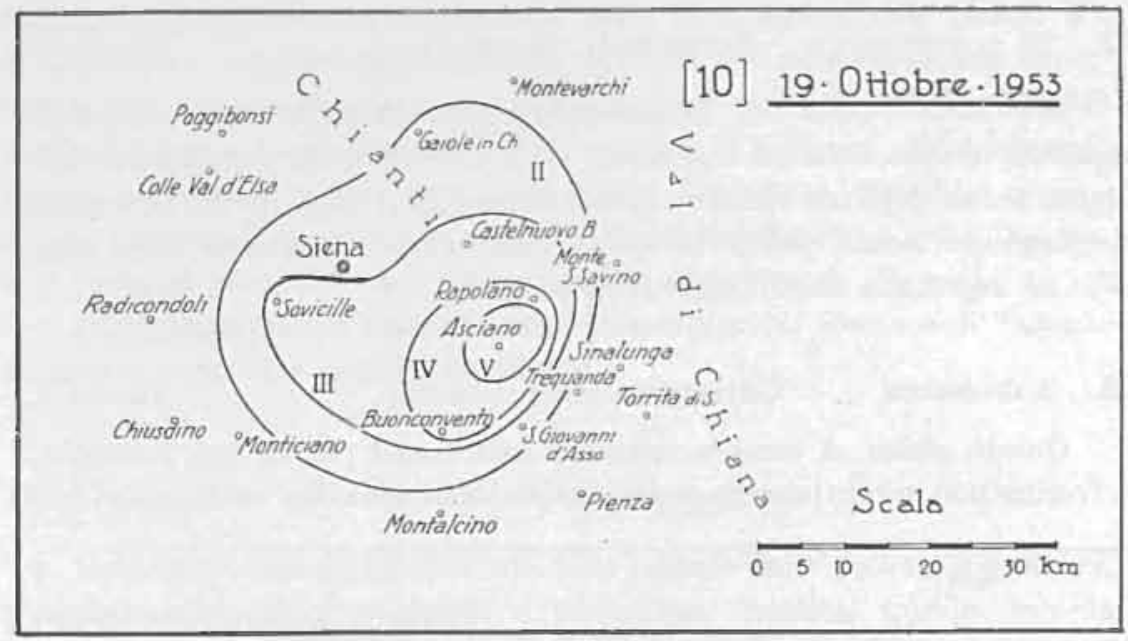

Fig. 7

\section{24 novembre}

Avezzano (L'Aquila)

Grado V

Nelle prime ore del mattino del 24 novembre 1953 , a circa $04^{\mathrm{h}} 13^{\mathrm{m}}$, la popolazione di Avezzano e quelle di altri paesi della Conca del Fucino venivano bruscamente svegliate da una forte scossa di terremoto accompagnata da un cupo boato.

Le informazioni macrosismiche che si sono potute avere sono insufficienti per il tracciamento delle curve isosiste. Possiamo solo dire che ad Avezzano la scossa raggiunse almeno il V grado, ebbe carattere prevalentemente sussultorio e una durata di 6-7 secondi. Di IV grado fu sentita a Collarmele e Celano; di III-IV in molte altre località ad occidente della Conca fucense, fra le quali Capistrello, Scurcola Marsicana, Tagliacozzo, e con la stessa intensità in alcuni paesi situati tra il Fucino e Sulmona, come Pescina e Cocullo.

Il movimento tellurico venne registrato nell'Osservatorio sismico di Roma i cui apparecchi segnarono l'inizio della perturbazione a $04^{\mathrm{h}} 13^{\mathrm{m}} 13^{\mathrm{s}}, 6$.

Sembra che altre due scosse, nella nottata, abbiano preceduto quella delle $04^{\mathrm{h}} 13^{\mathrm{m}}$ : la prima a circa $01^{\mathrm{h}}$ e l'altra a circa $03^{\mathrm{h}}$. Esse furono però di assai lieve entità e, data l'ora, passarono quasi inosservate. 
12. 25 novembre

Alle $14^{\mathrm{h} 26^{\mathrm{m}}}$ circa del 25 novembre 1953 fu avvertita a Stromboli una forte scossa tellurica che causo vivo allarme nella popolazione dell'Isola senza peraltro causare alcun danno. Si trattò di un fenomeno strettamente locale, poichè la scossa non fu avvertita nelle altre isole eolie nè registrata negli Osservatorî sismici.

13. 3 dicembre

Colletorto (Campobasso)

Grado V

Questo sisma ci sembra notevole non tanto per la sua intensità, in verità non molto elevata, quanto per la ubicazione della zona epi-

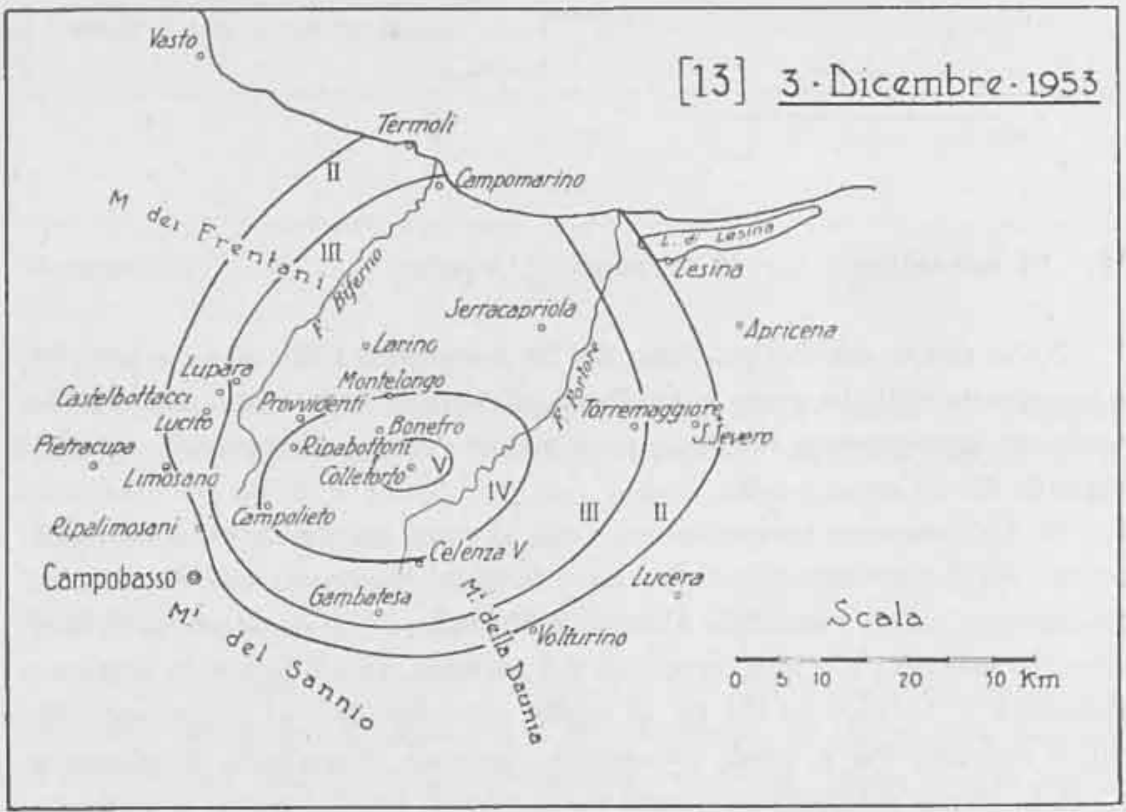

Fig. 8

centrale. Questa infatti, in base alle notizie macrosismiche a nostra disposizione, risulta situata fra le valli dei fiumi Biferno e Fortore, più precisamente fra Bonefro e Celenza Valfortore, e cioè in una zona che, nella Carta sismica del Baratta, figura ancora come stabile dal punto di vista sismico.

La figura 8 mostra l'estensione della propagazione macrosismica della scossa, estensione che può considerarsi abbastanza ampia se si tien 
conto dell'intensità non molto alta nella zona epicentrale (grado V). Il movimento tellurico risultò infatti sensibile alle persone su una superficie quasi circolare avente un diametro di cirea 60 chilometri. La ristreu̇̇a area epicentrale, la quale comprende il comune di Colletorto (Campobasso) risulta eccentrica su tale superficic poichè l'intensità deerebbe rapidamente verso sud, spegnendosi contro i Monti del Sannio e della Dauniä, e si propagó maggiormente verso nord in direzione di Termoli.

Dalle registrazioni ottenute negli Osservatorî di Roma e di Taranto si ricavò, come ora origine, $06^{\mathrm{n}} 5^{\mathrm{m}} 08^{\mathrm{s}}$.

\section{7 dicembre Radicondoli-Chiusdino (Siena)}

Grudo $\mathrm{Y}$

Epicentro nella parte orientale della regione delle Colline Metallifere, probabilmente fra Radicondoli e Chiusdino, località, queste, ove la scossa fu sentita con forte intensità.

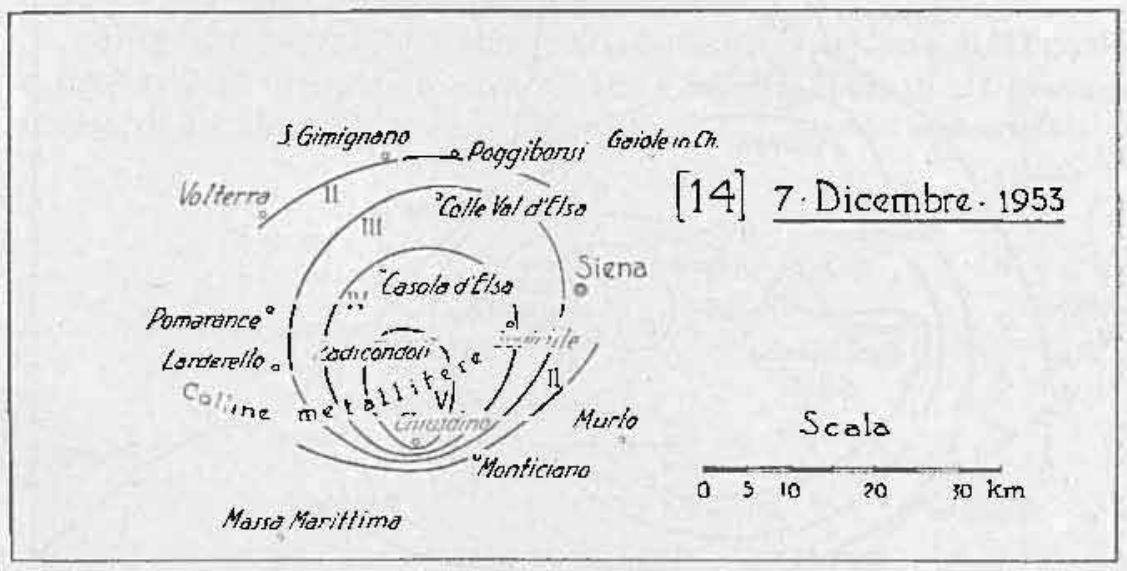

Fig. 9

La propagazione del moto tellurico fu caratterizzata, come mostrano le isosiste rappresentate in figura 9, da un rapidissimo decremento dell'energia sismica nelle direzioni SW, S e SE: a Monticiano, per esempio, che da Chiusdino dista poco più di 5 chilometri, la scossa non fu affatto avvertita. A Casole d'Elsa e Sovicille fu invece avvertita con intensità mediocre e a Colle Val d'Elsa con intensità leggera.

I'ora origine, dedotta dalle registrazioni ottenute a Siena, Firenze e Bologna, fu $10^{\text {ht }} 59^{\mathrm{m}} 17^{\mathrm{s}}$. 
15. 14 dicembre

Cesena (Forli)

Grado VI

Un notevole periodo sismico, che interessò buona parte del territorio compreso fra l'Appennino Emiliano e il Mare Adriatico, ebbe luogo nel mese di dicembre 1953.

\section{[15] 14. Dicembre 1953}

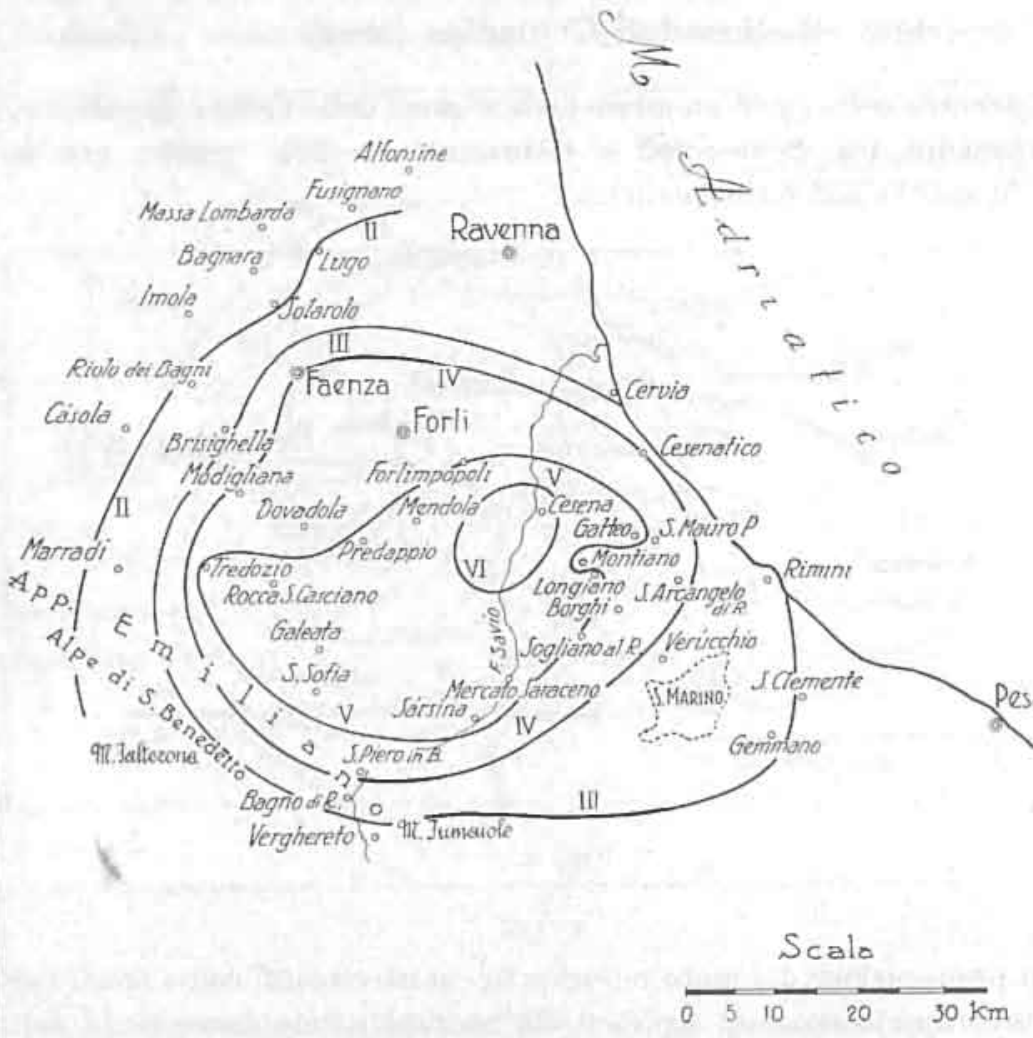

Fig. 10 
Come premonitoria può considerarsi una scossa avvenuta alle $22^{\mathrm{h}}$ circa del 3 dicembre ed avvertita di IV grado a Cesena. La scossa principale del periodo di verifico alle $08^{\mathrm{h}} 11^{\mathrm{m}}$ del 14 dicembre e il suo epicentro, in base alle registrazioni ottenute negli Osservatorî di Roma, Firenze, Padova, Bologna ed Oropa, fu determinato dall'I.N.G. nel punto di coordinate $44^{\circ} 04^{\prime} \mathrm{N}$ e $12^{\circ} 11^{\prime} \mathrm{E}$, cioè nella valle del Fiume Savio ad una decina di chilometri a sud di Cesena. In questa città la scossa raggiunse il grado VI Mercalli.

La zona urtata con forte intensità (grado $\mathrm{V}$ ) ha un contorno irregolare ed è limitata da un'isosista passante in prossimità di Forlimpopoli, Predappio, Tredozio, Sarsina, Sogliano al Rubicone, Montiano e Gatteo. Più regolari appaiono invece le isosiste dei gradi inferiori (fig. 10).

Una sensibile replica si ebbe il giorno successivo, 15 dicembre, alle $13^{\mathrm{h}} \mathrm{1}^{\mathrm{m}}$ circa. Essa fu avvertita con intensità di IV-V grado a Cesena e Meldola e con intensità di IV grado in numerose altre località, fra cui S. Pietro in Bagno, Sarsina e Mercato Saraceno.

Altre leggiere repliche si ebbero nei giorni successivi: una, di III grado a Cesena, il 17 dicembre a curea $05^{\mathrm{h}} 2^{\mathrm{m}}$ e un'altra, pure di III grado a Cesena, il 18 dicembre a circa $09^{\mathrm{h}} 18^{\mathrm{m}}$. 


\section{Anno 1954}

16. 6 febbraio

Valle Caudina (Benevento)

Grado V

Il 6 febbraio 1954 , alle $2^{\mathrm{h}} 55^{\mathrm{m}}$ circa, un terremoto di forte intensità (V grado) colpi Airola ed Arpaia, in provincia di Benevento, e Cervinara in provincia di Avellino.

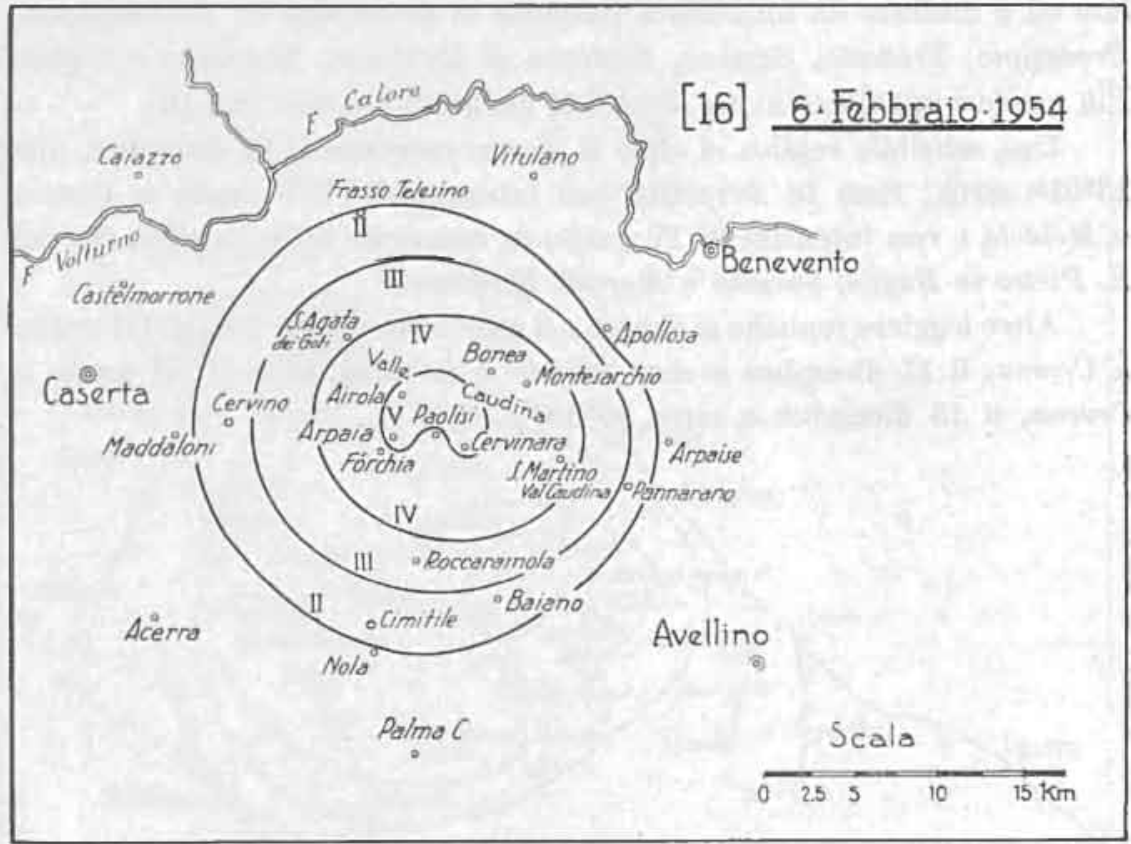

Fig. 11

In base alle notizie macrosismiche, l'epicentro è da porre nella Valle Caudina, in prossimità del punto di coordinate geografiche $41^{\circ} 03^{\prime} \mathrm{N}$ e $14^{\circ} 35^{\prime}$ E. La zona in cui la scossa fu avvertita macrosismicamente è alquanto ristretta ed ha una forma quasi circolare con un diametro di circa 25 chilometri (fig. 11).

L'area epicentrale di questo sisma è press'a poco la stessa del periodo sismico svoltosi nell'aprile del 1936, la cui scossa principale, molto forte, causò leggiere lesioni in diversi edifici di Airola. Nella Valle Caudina ebbe il suo epicentro anche una lunga ed intensa manifestazione tellurica 
verificatasi nella prima metà dell'anno 1903 e culminata il 4 maggio di quell'anno con una scossa rovinosa che arrecò danni gravi nei paesi di Arpaia e di Forchia $\left({ }^{3}\right)$.

\section{8 febbraio}

Teora (Avellino)

Grado V

Assai scarse sono le notizie che abbiamo potuto avere intorno a questo movimento tellurico, verificatosi a circa $05^{\mathrm{h}} 05^{\mathrm{m}}$ dell' 8 febbraio 1954. Sembra ch'esso sia risultato di forte intensità solo nel comune di Teora (Avellino), ove fu sentito da quasi tutti gli abitanti, ma non causò danni apprezzabili. Certamente si trattò di un fenomeno strettamente locale.

18. 23 marzo

Collestatte (Terni)

Grado VI

Pochi minuti prima delle $7^{\text {th }}$ del 23 marzo 1954, un terremoto valutato di VI grado Mercalli colpi Collestatte, frazione del comune di Terni, causando la caduta di qualche comignolo e leggiere fenditure in alcuni vecchi edifici.

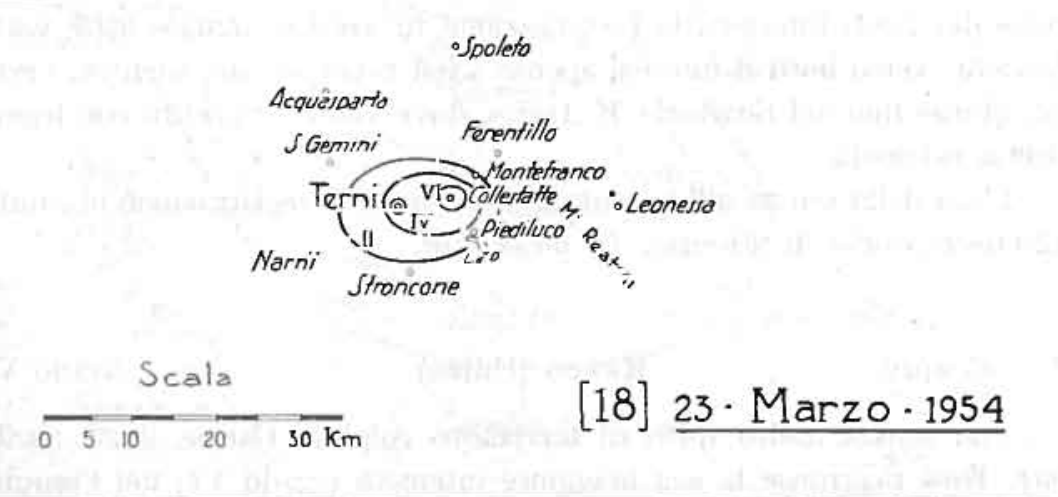

Fig. 12

La scossa, non registrata, secondo quanto ei risulta, in aleun Osservatorio, fu avvertita mediocremente nella città di Terni ed ebbe una propagazione limitatissima (fig. 12). La zona di percezione macrosismica ha la forma di un'ellisse con l'asse maggiore disposto in direzione EW e lungo una ventina di chilometri.

L'ora d'inizio del sisma, all'epicentro, sembra essere stata $06^{\mathrm{h}} 55^{\mathrm{m}}$ circa. Precedettero due scosse leggiere, avvertite da poche persone. Non risulta che si siano avute delle repliche. 
Poche notizie abbiamo potuto avere su questo sisma. Sappiamo solo che, a circa $15^{\mathrm{h}} 07^{\mathrm{m}}$ del 23 marzo 1954, ad Acireale fu sentita da una gran parte della popolazione una scossa che il relatore valutò del V grado Mercalli. A Mascali il movimento tellurico fu avvertito molto leggermente ed in molte località vicine ad Acireale passò del tutto inosservato.

20. 29 marzo

Grado V

Un forte movimento tellurico, notevole per la vastità della zona scossa macrosismicamente, ebbe luogo il mattino del 29 marzo 1954 nell'Appennino Emiliano.

L'area ove l'intensità raggiunse il V grado Merealli si estende, in direzione NS, da Modigliana a Galeata e comprende nel suo interno i paesi di Dovadola e Rocca S. Casciano (fig. 13). In queste località un forte rombo fu sentito contemporaneamente alla scossa.

Come mostrano le isosiste del sisma, alquanto irregolari specialmente quelle dei gradi inferiori, la propagazione fu assai disuguale nelle varie direzioni: verso nord il moto si spense assai rapidamente, mentre, verso sud, giunse fino nel territorio di Arezzo, dove venne avvertito con leggerissima intensità.

L'ora della scossa all'epicentro, dedotta dalla registrazione ottenuta nell'Osservatorio di Firenze, fu $06^{\mathrm{h}} 06^{\mathrm{m}} 10^{\mathrm{s}}$.

Una scossa molto forte di terremoto colpì la Carnia il 25 aprile 1954. Essa raggiunse Ia sua maggiore intensità (grado VI) nel Comune di Raveo, dove fu avvertita da tutta la popolazione con grande spavento. Caddero pezzi d'intonaco e si ebbero lievi danni negli edifici meno solidi. Ad Ampezzo la scossa fu d'intensità leggermente inferiore e produsse fenditure di poca entità in alcune abitazioni.

II movimento tellurico, come appare dalla fig. 14, interessò una regione molto vasta, comprendente buona parte delle province di Udine, di Belluno e di Treviso. La zona isosismica di VI grado risultò, in verità, alquanto limitata; quella di $\mathrm{V}$, però, si estese notevolmente verso ovest e verso sud-ovest includendo i territori di Auronzo, Lorenzago, Perarolo, Forno di Zoldo e Vittorio Veneto. 


\section{[20] 29-Marzo 1954}

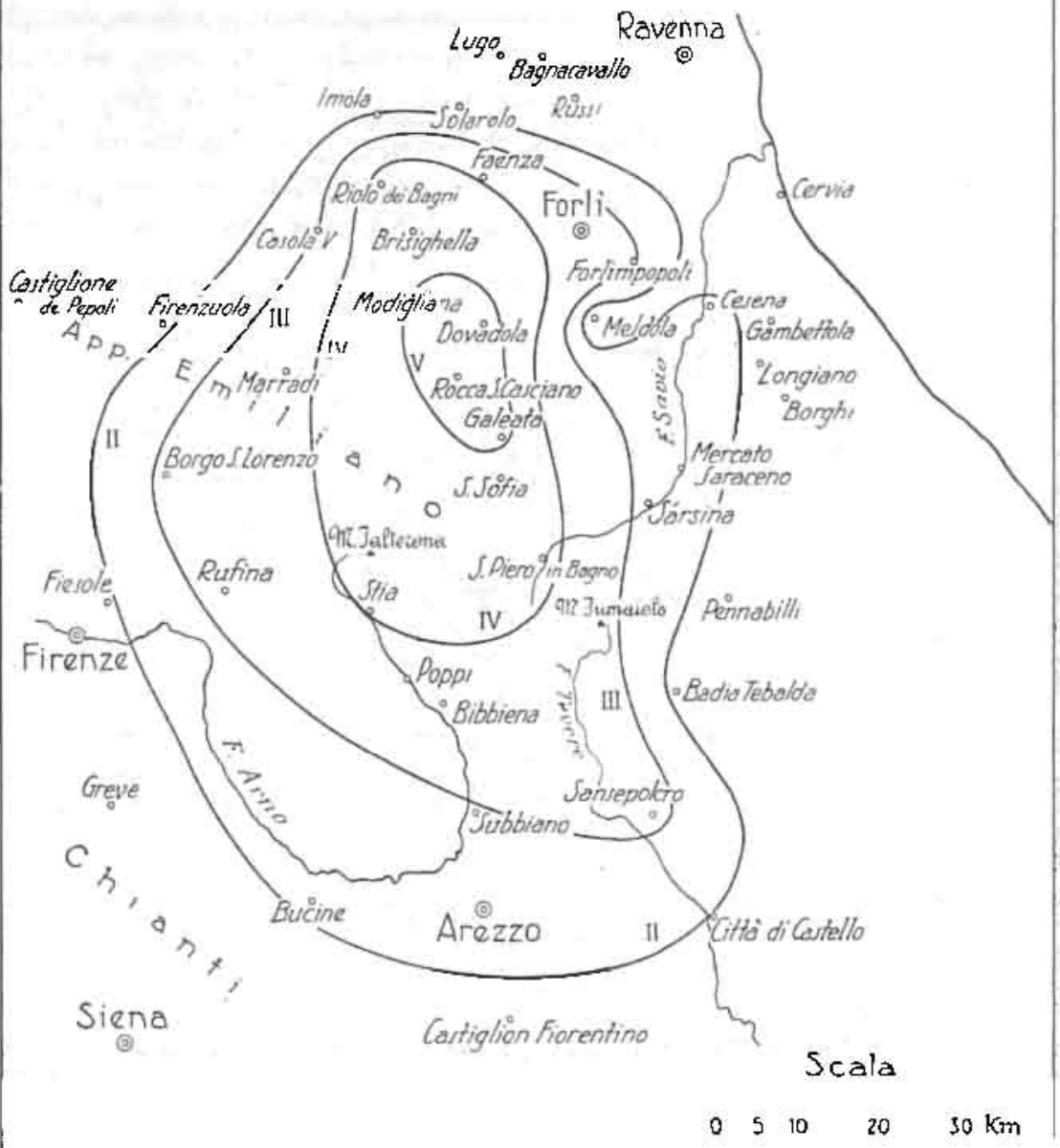

Fig. 13

Alquanto anomala fu inoItre la distribuzione delle intensità attorno alla zona epicentrale: verso nord infatti il moto sismico decrebbe con grande rapidità, mentre, verso ovest e specialmente verso sud, esso venne percepito a notevoli distanze dall'epicentro. A sud di Treviso, anzi, in una ristretta zona esterna alla isosista di II grado e comprendente i 
comuni di Dolo e di Mira (prov. di Venezia), la scossa venne avvertita con sensibile intensità.

La vastità della superficie macrosismica e l'irregolarità della propagazione del moto tellurico indicano una profondità ipocentrale piuttosto

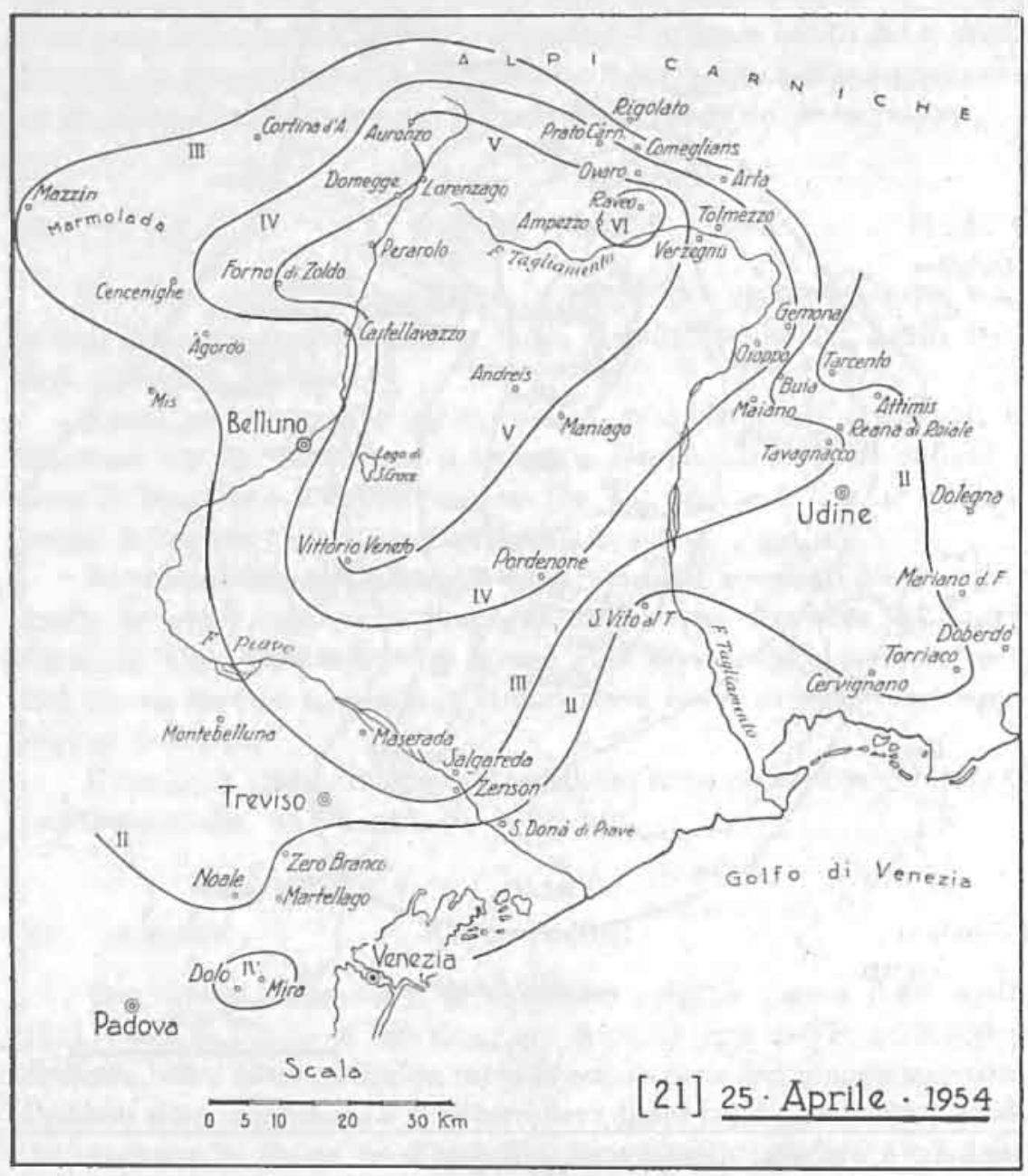

Fig. 14

notevole. Alle coordinate epicentrali, crediamo possano assegnarsi, con buona approssimazione, i valori $46^{\circ} 27^{\prime} \mathrm{N}$ e $12^{\circ} 50^{\prime} \mathrm{E}$; il tempo origine della scossa, in base alle registrazioni degli Osservatorî di Salò, Bologna, Firenze, Pavia, Oropa e Roma, può ritenersi $23^{\mathrm{h}} 17^{\mathrm{m}} 19^{\mathrm{s}}$. 
22. 9 maggio

Arcidosso (Grosseto)

Grado V

La regione del Monte Amiata, in Toscana, è nota per la sua grande attività sismica. Essa contiene, infatti, numerosi centri di scuotimento che frequentemente entrano in azione generando scosse che, pur essendo in genere di propagazione non molto estesa, raggiungono all'epicentro intensità talora rilevanti e cagionano gravi danni agli abitati dei paesi che circondano l'apparato vulcanico del Monte Amiata. Santa Fiora, Arcidosso, Radicofani, Abbadia S. Salvatore, Castel del Piano sono nomi che ricorrono assai spesso nelle cronache dei sismi d'Italia.

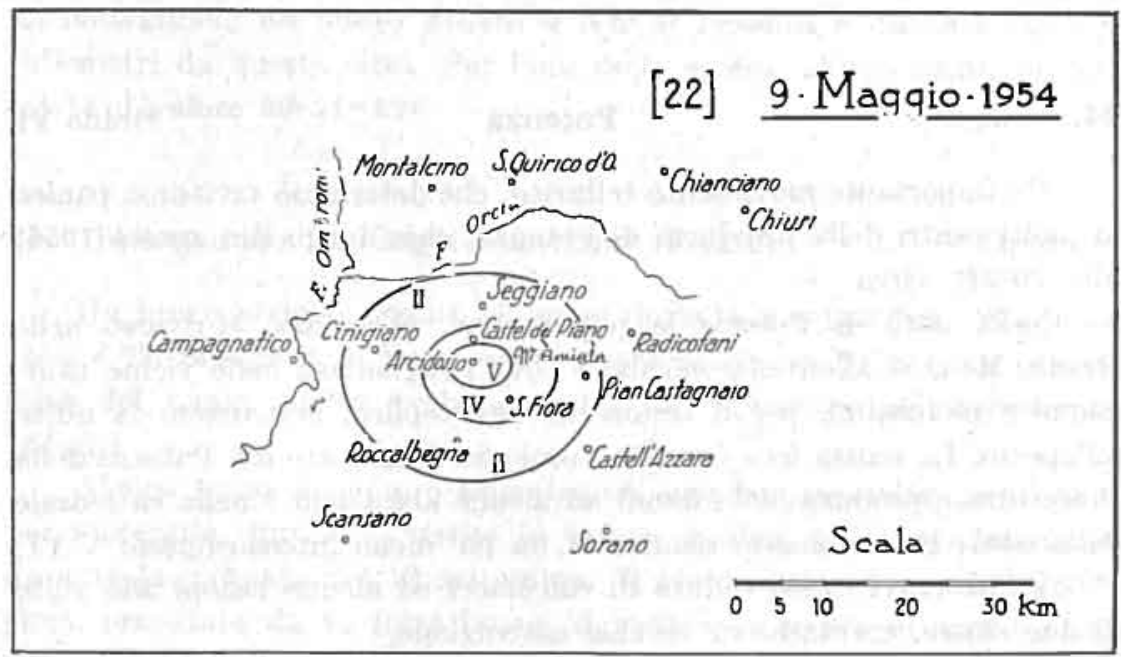

Fig. 15

La scossa del 9 maggio 1954 non fu di grande intensità, ma testimonia ancora una volta la instabilità sismica della regione amiatina. Essa raggiunse una forte intensità (grado V) ad Arcidosso, nei cui pressi è, con molta probabilità, da ubicare l'epicentro. Avvertito con forte rombo da tutta la popolazione, il movimento sismico causò, in varie case di Arcidosso, l'aggravamento di preesistenti lesioni.

La scossa ebbe una propagazione assai limitata (vedi fig. 15): a Santa Fiora e Castel del Piano fu avvertita con mediocre intensità; risultò leggerissima a Cinigiano, Roccalbenga, Pian Castagnaio e Seggiano.

L'ora d'inizio della scossa, dedotta dalla registrazione ottenuta nell'Osservatorio di Siena, fu $23^{\mathrm{h}} 34^{\mathrm{m}} 30^{\text {s }}$. 
Si tratta di una modesta scossa di carattere locale avvenuta a circa $01^{\mathrm{h}} 05^{\mathrm{m}}$ del 16 luglio 1954 e avvertita in una ristretta zona del versante padano dell'Appennino ligure. Essa fu sentita di V grado a Montereggio, frazione del Comune di Farini d'Olmo (Piacenza) e di IV nel vicinissimo abitato di S. Gregorio, frazione del Comune di Ferriere (Piacenza). In vicinanza di queste località fu con molta probabilità l'epicentro. Per quanto riguarda la propagazione, sappiamo solo che la scossa fu percepita con leggiera intensità, verso ovest, fino a Ferriere e, verso nord, fino a Béttola.

Un importante movimento tellurico, che determinò vivissimo panico in molti centri della provincia di Potenza, ebbe luogo il 6 agosto 1954, alle $20^{\mathrm{h}} 21^{\mathrm{m}}$ circa.

Nella città di Potenza la popolazione, allarmata, si riversò nelle strade. Molti si allontanarono dalla città rifugiandosi nelle vicine campagne e moltissimi, per il timore di una replica, trascorsero la notte all'aperto. La scossa fece fermare l'orologio della torre del Palazzo della Prefettura e produsse lievi danni ad alcune abitazioni e nella cattedrale della città. Il movimento sismico fu un po' meno intenso (grado V-VI) a Cancellara: ivi causò caduta di calcinacei ed alcune lesioni alle volte di due chiese, entrambe di vecchia costruzione.

Non è purtroppo possibile stabilire con sufficiente approssimazione quale sia stata la distribuzione delle intensità nella propagazione macrosismica della scossa. Possiamo solo dire che questa, almeno nelle direzioni $\mathrm{N}$ e NE, dovette essere alquanto vasta. Infatti, verso nord, la scossa giunse con intensità di IV e III grado rispettivamente fino a Canosa di Puglia (Bari) e a Cerignola (Foggia); verso nord-est, con intensità di III grado fino a Bari.

Possiamo dire anche, sulla scorta delle poche notizie pervenuteci, che la distribuzione delle intensità presentò parcechic anomalic. Così, per esempio, nella cittadina di Venosa, che è situata fra Potenza, dove la scossa fu di VI grado, e Cerignola, ove fu di II grado, il movimento tellurico non fu affatto avvertito. A Rionero, a sud del M. Vulture, fu di V-VI grado mentre ad Avigliano, situato fra Potenza e Rionero, fu appena di IV grado. 
Nell'attraversare l'apparato vuIcanico del Vulture, l'energia sismica subì un pressochè totale smorzamento: nella cittadina di Melfi, infatti, situata a nord del M. Vulture e distante da Rionero meno di una decina di chilometri, il movimento tellurico passò quasi inavvertito.

Verso NW la scossa giunse fin nelle province di Avellino e di Salerno, essendo stata avvertita con leggiera intensità nel Comune di Caposele (Avellino) e nei Comuni di Valva e di Laviano (Salerno).

Vastissima fu la propagazione della scossa sotto l'aspetto microsismico: essa venne registrata infatti in tutti gli Osservatorî italiani, da Messina e Reggio fino ad Oropa, ed anche in molti Osservatorî stranieri. Le coordinate epicentrali determinate dall'I.N.G. $\left(40^{\circ} 40^{\prime} \mathrm{N} ; 15^{\circ} 53^{\prime}\right.$ E) individuano un punto situato a NE di Potenza e distante circa 8 chilometri da questa città. Per l'ora della scossa, all'epicentro, fu calcolato il valore $20^{\mathrm{h}} 21^{\mathrm{m}} 12^{\mathrm{s}}$.

\section{14 settembre}

Cagli-Cantiano (Pesaro)

Grado V

Un breve periodo sismico si svolse durante il settembre 1954 nella zona della provincia di Pesaro circostante al Monte Nerone, in prossimità del quale, molto probabilmente, fu l'epicentro dei movimenti tellurici.

Alcune scosse leggiere o leggerissime, non ben precisate per l'ora e per l'intensità, furono avvertite in alcune località della zona predetta durante la giornata del 13 settembre. Il giorno successivo, alle $05^{\mathrm{h}} 08^{\mathrm{m}}$ circa, preceduta da un forte boato, si verificò la scossa principale, che nei comuni di Cagli e Cantiano fu sentita con maggiore intensità (V grado) e risultò prevalentemente sussultoria.

Diverse repliche di modesta intensità seguirono fino al giorno 19 settembre; una di esse, avvenuta alle $18^{\mathrm{h}} 30^{\mathrm{m}}$ circa del giorno 14 , fu avvertita di IV grado nei comuni di Piobbico ed Apecchio (Pesaro) ed un'altra, avvenuta alle $11^{\text {b }}$ circa del 19 settembre e con la quale sembra essersi chiuso il periodo sismico, fu avvertita di IV grado nel comune di Cantiano.

\section{11 ottobre}

Venzone (Udine)

Grado VI

Nel pomeriggio dell'11 ottobre 1954, una scossa tellurica molto forte si abbattè su tutto il Friuli raggiungendo in una zona piuttosto ampia il grado VI della Scala Mercalli. 
Anche per questo terremoto le notizie che si son potute raccogliere, troppo scarse in relazione alla vastità dell'area scossa, non hanno permesso il tracciamento delle curve isosiste. Ci risulta solo che nella zona colpita con intensità molto forte (VI grado), ove si verificarono lievi danni ad alcuni edifici, sono inclusi i comuni di Venzone, Tarcento, Colloredo e S. Daniele; che la scossa fu sentita fortemente (V grado) a Tolmezzo, Verzegnis, Vito d'Asio, Tricesimo, Gemona e Udine; mediocremente (IV grado) a Paularo; di III-IV grado a Pontebba e Tarvisio.

Verso sud il movimento si estinse assai rapidamente poichè, infatti, nei comuni di Latisana, S. Giorgio di Nogaro e Palmanova, tutti nella Bassa Friulana, esso passò quasi inosservato. Molto maggiore fu invece la propagazione verso SE, poichè a Trieste il sisma fu avvertito di III-IV grado.

Dalle registrazioni sismografiche ottenute negli Osservatorî di Tolmezzo, Salò, Oropa, Pavia, Bologna, Firenze e Roma, furono nell'I.N.G. determinate per le coordinate epicentrali i valori $46^{\circ} 20^{\prime} \mathrm{N}$ e $13^{\circ} 06^{\prime} \mathrm{E}$, i quali individuano un punto situato a 4 chilometri circa ad ovest di Venzone (Udine). L'ora della scossa all'epicentro fu $17^{\mathrm{h}} 45^{\mathrm{m}} 25^{\mathrm{s}}$. Fu potuta calcolare anche la "magnitudo " del sisma, per la quale risultò il valore 3,5 .

Il terremoto fu preceduto da tre lievissime scosse (verificatesi nella stessa giornata dell'11 ottobre alle $00^{\mathrm{h}} 48^{\mathrm{m}}, 10^{\mathrm{h}} 30^{\mathrm{m}}$ e $15^{\mathrm{h}} 19^{\mathrm{m}}$ rispettivamente) e seguito da numerosisime repliche che, a brevi intervalli di tempo l'una dall'altra, continuarono fino alle $8^{\text {h }}$ circa del 13 ottobre. Tutte queste repliche furono di leggera o leggerissima intensità, ad eccezione di una, verificatasi alle $00^{\text {h }} 43^{\mathrm{m}}$ circa del giorno 12 , che fu fortemente avvertita nel comune di Gemona del Friuli.

27. 20 novembre

Grisi (Palermo)

Grado VI

Il 18 novembre 1954, nel territorio di Grisi, frazione del comune di Monreale in provincia di Palermo, ebbe inizio un intenso periodo sismico che, per la frequenza delle scosse, per i forti boati che le accompagnarono e per le gravi lesioni causate a molte abitazioni, mise in istato di vivissimo allarme la popolazione di quel piccolo centro. Dopo qualche giorno dall'inizio dei fenomeni sismici, continuando essi con grandissima frequenza, gli abitanti, presi da panico, sfollarono in massa da Grisi rifugiandosi nei paesi vicini e solo dopo molti giorni, quando le scosse cominciarono a diradarsi, s'indussero, poco a poco, a rientrare nelle loro abitazioni. 
Il periodo sismico cominció con una lieve scossa premonitoria avvertita dalla maggior parte della popolazione del solo centro di Grisi alle $10^{\mathrm{h}}$ circa del 18 novembre 1954.

Il 20 novembre, alle $06^{1 \mathrm{1}} 35^{\mathrm{m}}$, accompagnata da forte rombo, si verifico la scossa principale che nelle vicinanze di Grisi spiegó la massima intensità, valutabile del VI grado Mercalli. Fu infatti questa località

\section{[27] 20. Novembre 1954}

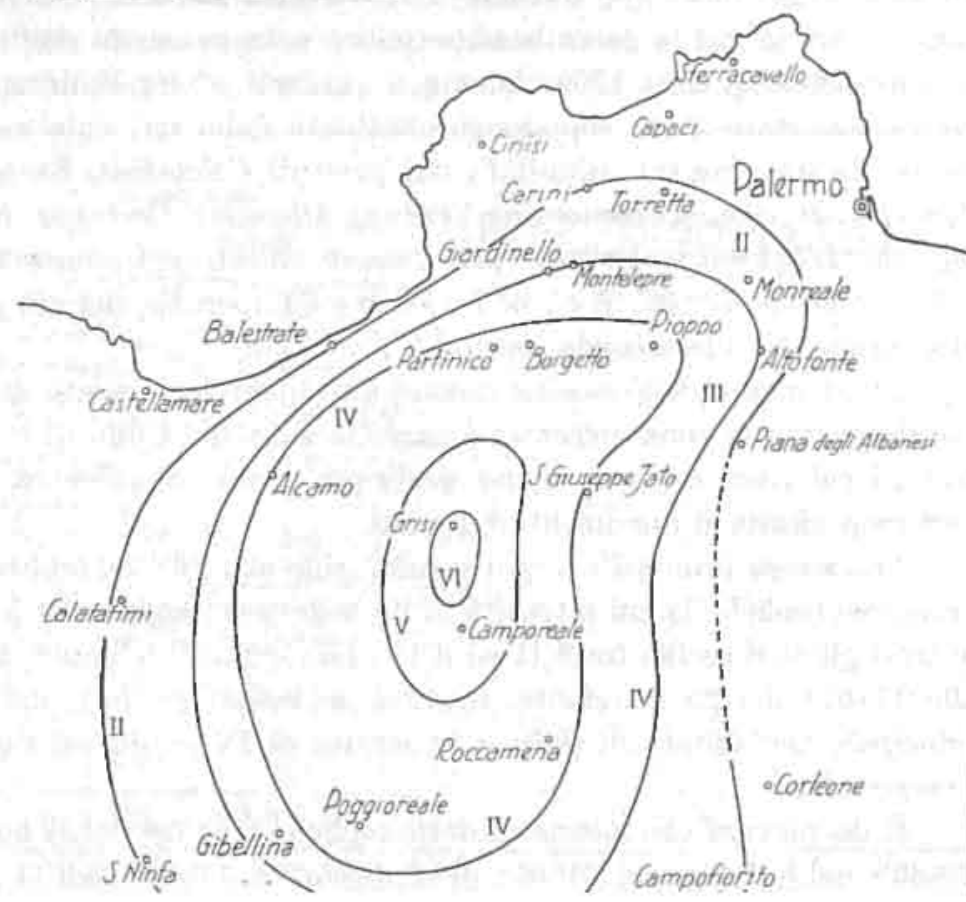


che ebbe a sperimentare i maggiori effetti dinamici della scossa: fenditure numerose ma leggere in case di recente costruzione; notevoli in case vecchie e poveramente costruite.

A Camporeale, che in linea d'aria dista all'incirca una mezza dozzina di chilometri da Grisi, il movimento tellurico fu fortemente sentito, ma non causò altri danni all'infuori di lievi fenditure in abitazioni di difettosa costruzione; la sua intensità, in quel paese, può ritenersi essere stata del V grado Mercalli.

La scossa, che fu registrata solo negli Osservatorî di Palermo e di Messina, ebbe una propagazione macrosismica alquanto ristretta. La zona infatti in cui la perturbazione tellurica fu percepita dall'uomo ha una superficie di circa 1500 chilometri quadrati ed è racchiusa, come fu potuto accertare in un sopraluogo effettuato dallo scrivente nella zona stessa, da una isosista delimitata dai paesi di Calatafimi, Santa Ninfa, Sambuca di Sicilia, Bisacquino, Piana Albanese, Carini e Balestrate (fig. 16). L'epicentro si ritiene possa essere ubicato nel punto di coordinate $37^{\circ} 56^{\prime} \mathrm{N}, 13^{\circ} 06^{\prime} \mathrm{E}$ e cioè fra Grisì e Camporeale, ma più prossimo alla prima che alla seconda località.

L'andamento delle isosiste mostra un rapido decremento della energia sismica dalla zona epicentrale verso la zona del Golfo di Castellammare, i cui paesi costieri, anche quelli più vicini all'epicentro, non avvertirono affatto il movimento tellurico.

Alla scossa principale fecero seguito, sino alla fine del febbraio 1955 , numerose repliche la cui intensità in un solo caso raggiunse il V grado e in tutti gli altri oscillò fra il II ed il IV. La replica di V grado, avvenuta alle $11^{\text {h }} 07^{\mathrm{m}}$ del 25 novembre, aggravo le lesioni prodotte dalla scossa principale nell'abitato di Grisi e fu sentita di IV grado nel Comune si Camporeale.

$\mathrm{E}$ da rilevare che alcune di dette repliche $\left(19^{\mathrm{h}} 52^{\mathrm{m}}\right.$ del 30 novembre;

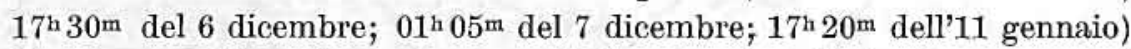
furono avvertite nel solo comune di Camporeale, ciò che fa pensare ad un lieve spostamento dell'epicentro verso sud.

Più che per 1 ı sua entità, questo periodo sismico è notevole perchè non risulta che la zona di Grisì sia stata anteriormente sede di altre manifestazioni sismiche corocentriche.

Riguardo alla causa delle scosse, puó pensarsi che esse siano state provocate da piccoli movimenti di assestamento lungo qualche faglia forse esistente in prosecuzione di quella visibile sui due versanti della dorsale Monte Jato-La Cometa. In ogni modo l'intensità molto forte nella zona epicentrale circondante l'abitato di Grisì e la modesta propa- 
gazione delle scosse, anche di quella principale, inducono a ritenere che la profondità dell'epicentro sia stata molto piccola; e probabilmente trattasi di un ipocentro rivelatosi per la prima volta o almeno risvegliatosi dopo un lungo periodo di inattività.

\section{21 dicembre Campli (Termo)-Ascoli Piceno Grado V}

Alle $2^{\text {11 }} 30^{\text {th }}$ cirea del 21 dicembre 1954 , la popolazione della cittadina di Campli, in provincin di Teramo, fo improvvisamente destata da un intenso boato seguito quasi immediatamente da una forte scossa di terremoto (V grado) durata circa $5^{\text {s }}$ e di carattere prevalentemente

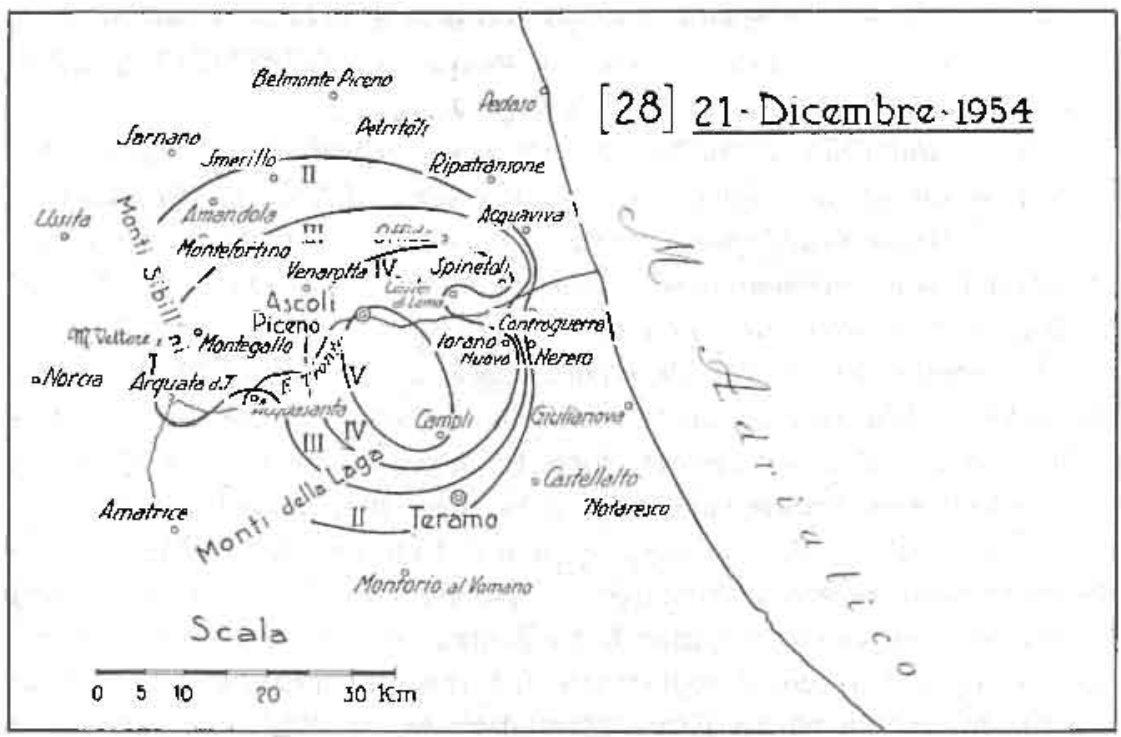

Fig. 17

sussultorio. Con quasi nguale intensita, ma prima ondulatorio e poi sussultorio, il movimento tellurico fu avvertito in quella parte della Valle del 'lronto che circonda Ascoli Pieno. Tra questa città e Campli molto probabilmente fu l'epicentro del sisma.

L'isosista racchiudente l'area scossa con sensibile intensità (IV grado) presenta verso $\mathrm{E}$ un andamento alquanto irregolare: comprende infatti i comuni di Spinetoli e ITorano Nuovo lasciando fuori il comune di Castel di Lama, più vicino dei primi due alla zona epicentrale (vedi fig. 17). 
Per quanto non si abbiano notizie per poter stabilire l'andamento della isosista di II grado ad W e SW della zona epicentrale possiamo dire che la propagazione del sisma non fu molto ampia. Verso E il movimento decrebbe molto rapidamente si da passare inosservato ad Acquaviva, Controguerra e Nereto; più estesamente si propagò verso $\mathrm{W}$ così da essere ancora avvertito con leggera intensità nel Comune di Arquata del Tronto.

29. 27 dicembre

Salina (Isole Eolie)

Grado VII

Un periodo sismico di frequenza assai elevata e culminato in una scossa di fortissima intensità si svolse durante il mese di dicembre 1954 nelle Isole Eolie, colpendo gravemente Salina, la seconda, per estensione e popolazione, fra le isole dell'Arcipelago.

Fra le numerose perturbazioni telluriche abbattutesi su Salina durante il secolo attuale, questa, del 1954, è senza dubbio la più violenta, ove si accettui il sisma quasi disastroso del 17 agosto 1926 che nei centri abitati dell'Isola, specialmente a Malfa e Pollara, provocò crolli e lesioni assai gravi in moltissime abitazioni.

Il periodo sismico del 1954 cominciò a manifestarsi l'11 dicembre, alle $13^{\mathrm{h}} 47^{\mathrm{m}}$ curca, con una seossa che fu anche registrata negli Osservatorî di Messina e di Reggio Calabria. Altre tre sensibili scosse, pure registrate nei predetti Osservatori, si ebbero il 14 dicembre, dopo le quali parve ritornata la calma. Ma alle $16^{\mathrm{h}} 41^{\mathrm{m}}$ eirca del giorno 23 , a Salina, il suolo cominciò nuovamente a tremare e, a partire da tale ora, i movimenti tellurici si susseguirono con grande frequenza fino alle $07^{\mathrm{h}} 30^{\mathrm{m}}$ del giorno successivo, addensandosi soprattutto intorno alla mezzanotte fra il 23 e il 24. Dopo una nuova pausa di relativa tranquillità, alle prime luci dell'alba del 27 dicembre, la popolazione dell'Isola veniva svegliata di soprassalto da una sensibile scossa accompagnata da intensi boati. Alle $08^{\mathrm{h}} 59^{\mathrm{m}}$ circa si verifico la scossa principale che investì con grande violenza soprattutto quella parte dell'Isola che è dominata dall'antico vulcano chiamato Monte dei Porri (fig. 18 b). Il centro più colpito, fra i cinque in cui è divisa la popolazione di Salina, fu Pollara, piccolo villaggio, frazione del comune di Malfa, che sorge sul fianco $\mathrm{NW}$ del Monte dei Porri: alcune vecchie case crollarono in parte e numerose altre restarono gravemente danneggiate. Fortunatamente non vi furono vittime, ma la popolazione, presa da vivissimo panico, abbandonò le abitaziom accampandosi sulla riva del mare. 
Anche a Malfa, il centro più popolato dell'Isola, situato al centro della costa nord, e nel comune di Leni, a sud del Monte dei Porri, la scossa fu assai violenta e causò lesioni gravi in molte abitazioni.

L'intensità del sisma, a Pollara e nelle immediate vicinanze, può essere valutata di VII grado Merealli, anche se gli effetti dinamici sulle abitazioni possono indurre a giudicarla ancora più alta. $\mathrm{E}$ da tener presente che le case di Pollara, come pure quelle degli altri centri di Salina, sono in generale poco solide sia perchè di costruzione assai difettosa sia perchè oramai molto vecchie ed indebolite da precedenti fenomeni sismici.

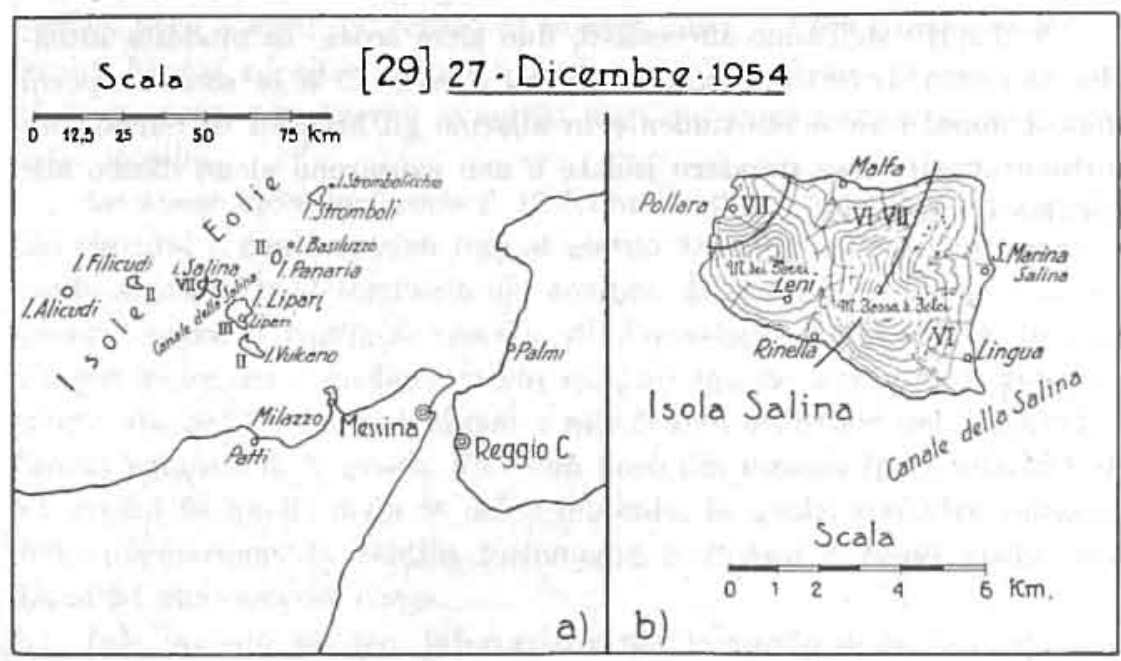

Fig. 18

D'intensità inferiore la scossa risultò sulla costa orientale dell'Isola, ove sorgono S. Marina Salina e il paesetto di Lingua. Ma anche in queste località si verificarono lesioni più o meno gravi in molti edifici.

Abbiamo assai scarse notizie sull'entità della scossa nelle altre isole dell'Arcipelago. Possiamo solo dire che nella vicina Lipari il movimento tellurico fu generalmente avvertito, ma non desto alcuna apprensione: ancora più leggermente fu avvertito nelle isole di Panarea, Filicudi e Vulcano (fig. 18 a). Sotto forma microsismica, la scossa potè essere registrata, oltre che negli Osservatori di Messina e di Reggio Calabria, anche in quelli di Taranto e di Roma.

All'epicentro della scossa, situato nelle immediate vicinanze di Pollara, possono essere assegnati, come coordinate geografiche, i valori 
$38^{\circ} 35^{\prime} \mathrm{N}$ e $14^{\circ} 48^{\prime} \mathrm{E}$. Come ora origine, può essere assunto il valore $08^{\mathrm{h}} 58^{\mathrm{m}} 55^{\mathrm{s}}$.

Durante la stessa giornata del 27 dicembre, a brevissimi intervalli di tempo l'una dall'altra, seguirono numerosissime repliche di varia entità. Alcune di esse (quelle delle $09^{\mathrm{h}} 20^{\mathrm{m}}, 11^{\mathrm{h}} 07^{\mathrm{m}}, 11^{\mathrm{h}} 15^{\mathrm{m}}$ e $15^{\mathrm{h}} 28^{\mathrm{m}}$ ) furono registrate negli Osservatorî di Messina e di Reggio Calabria; più delle altre intensa fu quella delle $11^{\mathrm{h}} 15^{\mathrm{m}}$ che venne registrata anche a Taranto $\mathrm{e}$ a Roma.

Nei giorni successivi le repliche andarono diminuendo d'intensità sempre più distanziandosi nel tempo e cessarono del tutto il 31 dicembre 1957.

Nell'aprile dell'anno successivo, due altre scosse di modesta intensità, la prima avvertita alle $00^{\mathrm{h}} 40^{\mathrm{m}}$ del giorno 12 e la seconda pochi minuti dopo, misero nuovamente in allarme gli abitanti di Salina. Ma fortunatamente esse rimasero isolate e non causarono alcun danno alle persone e alle cose. 


\section{Anno 1955}

29. 2 febbratio

Pedara (Catania)

Grado $Y$

Un periodo sismico, le cui manifestazioni interessarono una zona assai ristretta del versante meridionale dell'Etna, ebbe luogo durante il gennaio e, principalmente, durante il febbraio del 1955 .

Lisso s'iniziò, sembra, con alcune lievissime scosse avvertite a Pedara il 6 gennaio, aile quali seguirono, nei giorni 18 e 19 dello stesso mese, altre scosse, pure lievissime e di carattere strettamente locale, avvertite, alenne, poco a nord del centro di Pedara, aitre a Fleri (frazione di Zafferana Etnea) ed altre ancora a Zafferana Etnea. Dopo di esse, dal 20 al 31 gennaio, non furono avvertiti altri movimenti tellurici neile predette località.

Le scosse ricominciarono il $1^{\circ}$ febbraio, più frequenti e d'intensità più elevata, e continuarono fino al giorno 18 dello stesso mese interessando soprattutto il territorio del comune di Pedara ed anche, ma alquanto meno, il contiguo comune di Trecastagni. Quasi tutte furono d'intensità leggera o mediocre; solo quattro (quelle avvenute rispettivamente aile $5^{\text {h }} 2^{\text {mh }}$ del $1^{0}$ febbraio e alle $5^{\mathrm{h}} 45^{\mathrm{m}}, 6^{\mathrm{h}}, 6^{\mathrm{t}} 30^{\text {m }}$ del 2 febbraio) furono valutate di V grado. Forse un poco più intensa (però inferiore al VI grado) fu quelia delle $6^{\text {h }}$ del 2 febbraio, la quale, con vivo allarme della popolazione, fu sentita fortemente a Pedara e causó anche dei danni ad una casa del paese.

Tale periodo sismico, interessante per io studio della sismicità del versante meridionale dell'Etra, è stato ampiamente esaminato in una nota di S. Cocuzza Silvestri pubblicata nel volume IX degli Annali di Geofisica $\left({ }^{4}\right)$. Da essa abbiamo tratto le notizie che precedono e ad essa si rimanda per maggiori particolari suil'argomento.

30. 9 febbraio

31. 4 marzo
Monte S. Angelo (Foggia) idem
Grado VII-VIII Grado VI

La regione d'Italia più colpita da sismi durante il quinquennio 195̄31957 fu certamente la regione garganica.

Questo gigantesco massiccio caleareo, ricco di doline, di caverne e di. grotte che ne rivelano la natura carsica, è purtroppo ricco altresì di centri sismici attivi, situati in parte anche sotto il fondo del mare che ne 
lambisce le alte e ripide coste. Da essi, in ogni tempo e con rilevante frequenza, sono stati irraggiati movimenti tellurici spesso fortissimi, talora anche rovinosi.

Abbiamo già parlato del sisma avvertito con forte intensità nella zona compresa fra S. Giovanni Rotondo e Manfredonia il 19 luglio 1953 (n. 4). Per avere un quadro più completo dell'attività sismica svoltasi nella regione garganica durante il periodo di tempo che abbiamo preso in esame, sarà bene ricordare anche due manifestazioni telluriche avutesi nel 1954 e i cui epicentri furono probabilmente nel mare prospiciente la costa orientale del Gargano.

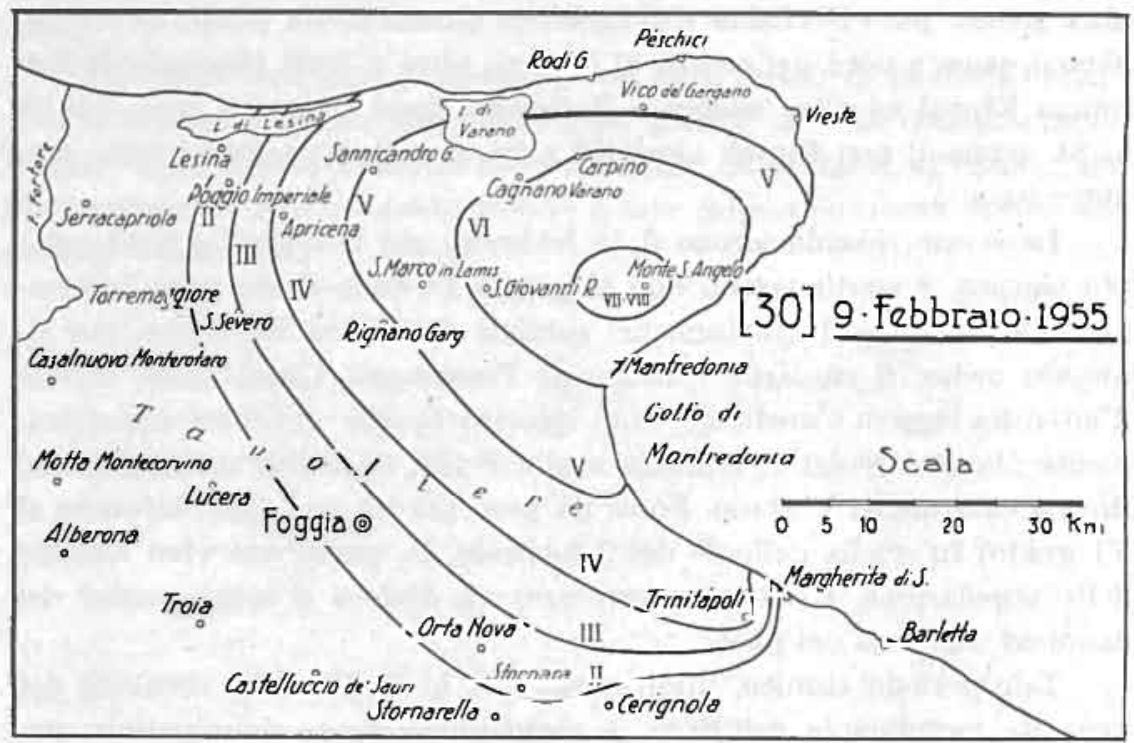

Fig. 19

La prima avvenne alle $01^{\mathrm{h}} 13^{\mathrm{m}}$ circa del 13 aprile e fu avvertita a Vieste con intensità di IV grado. Non ci risulta però ch'essa sia stata percepita sensibilmente anche in altre località del Promontorio. La seconda, avvenuta alle $03^{\mathrm{h}} 25^{\mathrm{m}}$ circa del 26 ottobre, fu un po' più intensa e di una propagazione molto più vasta della prima. Venne infatti sentita di IV-V grado a Vieste, risultò sensibile in tutto il Gargano e giunse con un'intensità di III-IV fino a S. Severo, Lucera e Stornara.

Ben più intensa che negli anni 1953 e 1954 fu, nel Gargano, l'attività sismica durante il 1955 .

Il 9 febbraio, alle $11^{\mathrm{h}} 07^{\mathrm{m}}$ circa, preceduta da un fortissimo boato, una scossa d'intensità quasi rovinosa (VII-VIII grado) si abbattè sulla 
zona meridionale del Promontorio colpendo specialmente la cittadina di Monte S. Angelo, la cui popolazione si riversò nelle piazze e nelle strade in preda al terrore.

Assai gravi furono, nella cittadina, i danni prodotti dalla scossa: moltissime abitazioni rimasero seriamente lesionate ed alcune furono dichiarate inabitabili; nelle campagne circostanti, in diversi caseggiati rurali di vecchia e difettosa costruzione si verificarono crolli, fortunatamente senza vittime umane. Danni di una certa gravità subirono le chiese di Monte S. Angelo dedicate a S. Francesco e a S. Benedetto.

A Monte S. Angelo e nelle sue vicinanze, la scossa fu avvertita prevalentemente come sussultoria ed ebbe la durata di una decina di secondi. Fra i fenomeni concomitanti fu segnalato, oltre al pauroso boato, un sensibile sollevamento dell'acqua nei pozzi delle campagne.

La zona isosismica ove il movimento tellurico fu sentito con intensità molto forte (VI grado) abbraccia una vasta parte della Montagna garganica ed include i comuni di S. Giovanni Rotondo, Manfredonia e Carpino. Le isosiste rappresentate in fig. 19 mostrano la propagazione macrosismica della scossa, la quale fu avvertita con intensità di $\mathrm{V}$ grado a Sannicandro Garganico, S. Marco in Lamis, Cagnano Varano e. con intensità di IV grado, nei comuni di Vieste, Peschici, Rodi, Vico del Gargano, Apricena, Rignano Garganico e Trinitapoli, tutti della provincia di Foggia.

Si ebbero registrazioni in tutti gli Osservatorî italiani. Da esse, nell'I.N.G., furono dedotti, per le coordinate epicentrali, i valori $41^{\circ} 42^{\prime} \mathrm{N}$ e $15^{\circ} 53^{\prime} \mathrm{E}$, i quali individuano un punto situato ad ovest di Monte S. Angelo e distante dalla cittadina circa 6 chilometri. L'ora della scossa all'epicentro fu $11^{\mathrm{h}} 06^{\mathrm{m}} 57^{\mathrm{s}}$.

Per tutto il mese di febbraio, quasi ogni giorno, seguirono scosse di leggera intensità. Una, un po' più intensa delle altre (IV-V grado), fu avvertita il 24 febbraio alle $12^{\mathrm{h}} 20^{\mathrm{m}}$ circa.

Il 4 marzo, una nuova scossa molto forte (VI grado), generata dallo stesso ipocentro ed anch'essa preceduta da fortissimo rombo, aggravò sensibilmente a Monte S. Angelo i danni provocati dalla scossa del 9 febbraio. Fu registrata negli Osservatorî di Taranto, Roma, Messina e la sua ora origine può ritenersi $18^{\mathrm{h}} 36^{\mathrm{m}} 30^{\mathrm{s}}$.

Altre repliche si ebbero durante il mese di marzo. Esse si mantennero di lieve intensità fino al 18 di marzo, giorno in cui tutto il Gargano fu scosso nuovamente da un fortissimo movimento tellurico. Di questo parleremo al n. 33 . 
Il 12 marzo 1955, alle $7^{\mathrm{h}} 57^{\mathrm{m}}$, fu avvertita a Medesano e a Noceto, in provincia di Parma, una scossa piuttosto forte di terremoto, della durata di 7 secondi e di carattere sussultorio.

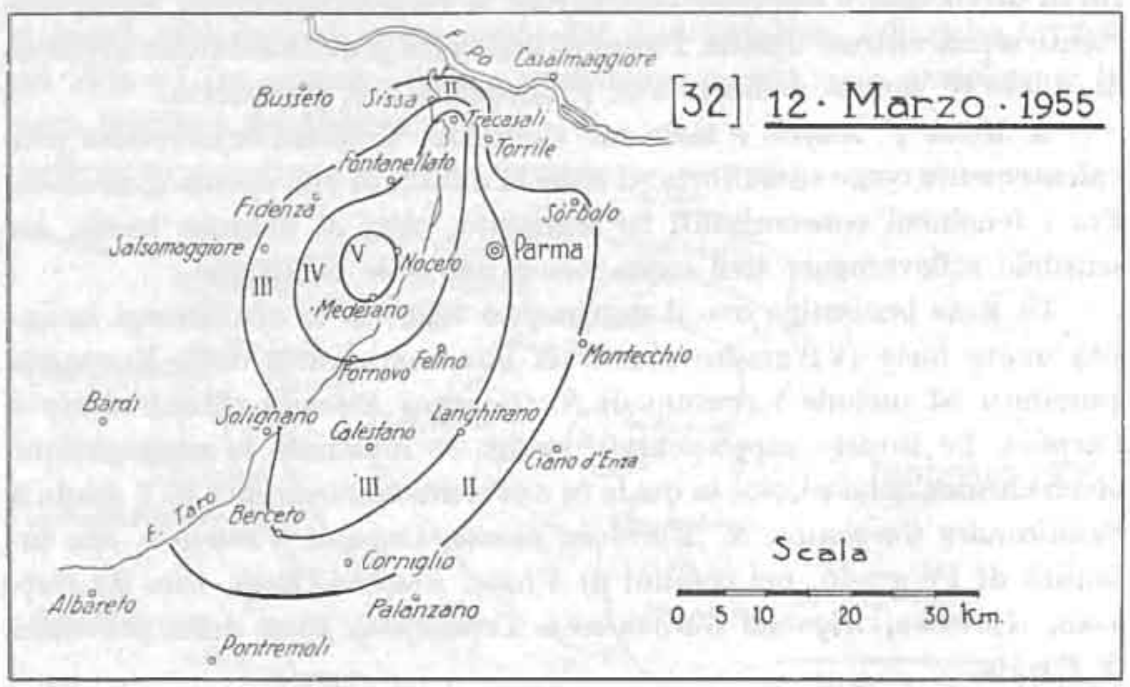

Fig. 20

II movimento tellurico, registrato in alcuni Osservatorî italiani, fu avvertito con mediocre intensità in un'area non molto ampia estendentesi, Iungo Ia valle del Taro, all'incirca da Fornovo fino a Trecasali (v. fig. 20). La zona isosismica di leggera intensità è delimitata da una linea che include la città di Parma e diversi comuni della sua Provincia, fra i quali Sissa, Fidenza, Felino, Berceto e Fontanellato. L'epicentro della scossa, cui possono assegnarsi le coordinate $44^{\circ} 48^{\prime} \mathrm{N}$ e $10^{\circ} 06^{\prime} \mathrm{E}$, cade a NW di Medesano.

Una sensibile replica si ebbe alle $19^{\mathrm{h}} 45^{\mathrm{m}}$ circa del 22 marzo.

33. 18 marzo

Monte S. Angelo (Foggia)

Grado VI-VII

Un'altra scossa, come si è precedentemente accennato, la più intensa dopo quella del 9 febbraio, si verificò nella regione garganica il 18 marzo 1955 causando altri danni alle costruzioni e nuovo sgomento nelle popo- 
lazioni dei centri già duramente colpiti. Essa avvenne alle $07^{\mathrm{u}} 49^{\mathrm{m}} 44^{\mathrm{s}} \mathrm{e}$ fu registrata in tutti gli Osservatorì italiani.

L'epicentro fu ancora fra Monte S. Angelo e San Giovanni Rotondo, nelle immediate vicinanze della Valle Carbonara.

In base alle notizie a nostra disposizione, anche se, in verità, piuttosto scarse, ci sembra di poter affermare che questa replica, pur essendo

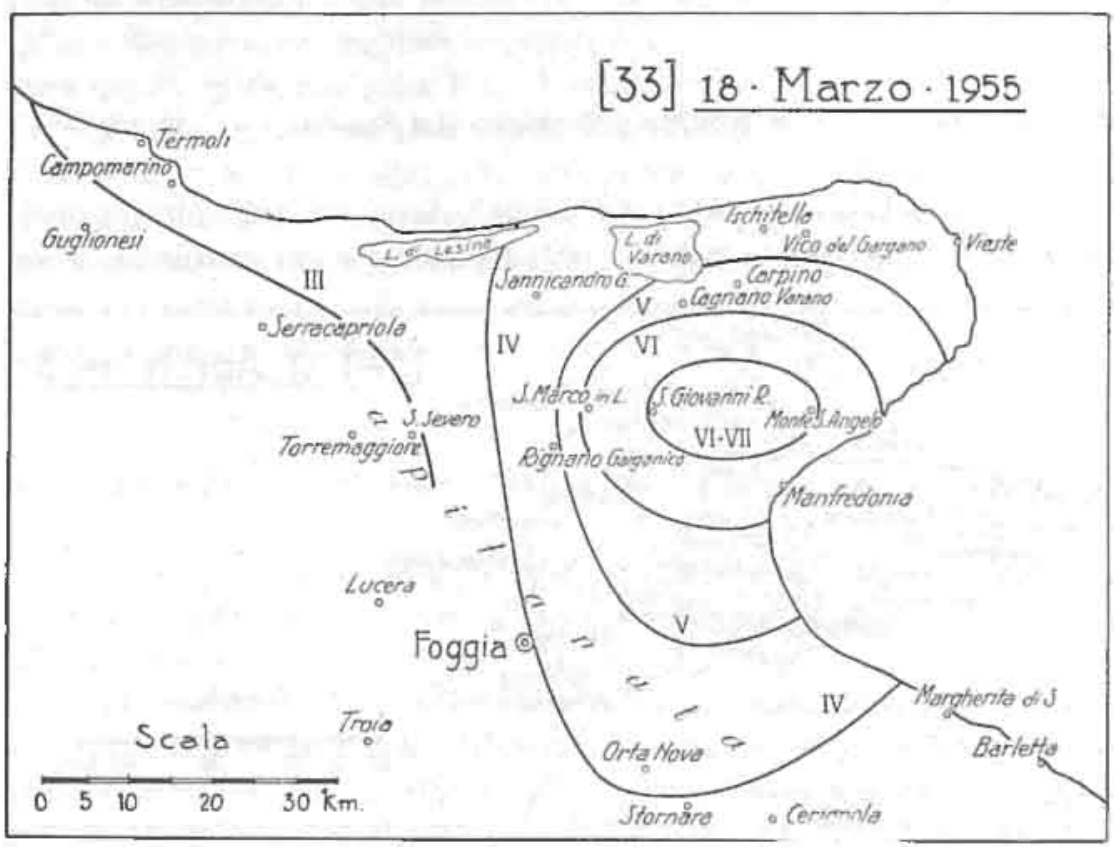

Fig. 21

stata d'intensità inferiore a quelia del terremoto del 9 febbraio, ebbe, forse a causa di una più accentuata profondità ipocentrale, una propagazione alquanto più vasta. Essa infatti, con leggera intensità, fu avvertita, fin oltre Termoli, in tutta la fascia costiera molisana e, nel Tavoliere di Puglia, si estese con sensibile intensità fin poco oltre Orta Nova (vedi fig. 21). Anche la zona di maggior scuotimento fu alquanto più ampia poichè in essa si trovó inclusa, oltre a Monte S. Angelo, anche la cittadina di San Giovanni Rotondo. In questi due centri, ove l'intensità raggiunse il VI-VII grado, molti edifici già provati dai precedenti movimenti tellurici, riportarono nuovi e sensibili danni. Vi fu anche qualche crollo di case coloniche, già fortemente lesionate e, per precauzione, già in precedenza sloggiate dagli abitantí. 
Nel comune di S. Marco in Lamis, la scossa, sentita molto fortemente (VI grado) e con grande spavento dalla popolazione, causò nuove fenditure in molte case ed anche in qualche edificio pubblico. Il movimento tellurico, che nella zona megasismica ed in quella racchiusa dalla isosista di VI grado fu accompagnato da forte rombo, risultò sussultorio a Monte S. Angelo, S. Giovanni Rotondo e S. Marco in Lamis; ondulatorio nelle altre località.

34. 11 aprile La Spezia - Sarzana (La Spezia)

II giorno 11 aprile 1955 , alle $16^{\mathrm{b}} 24^{\mathrm{m}}$ circa, si verificò in provincia di La Spezia una scossa tellurica abbastanza forte che raggiunse la sua

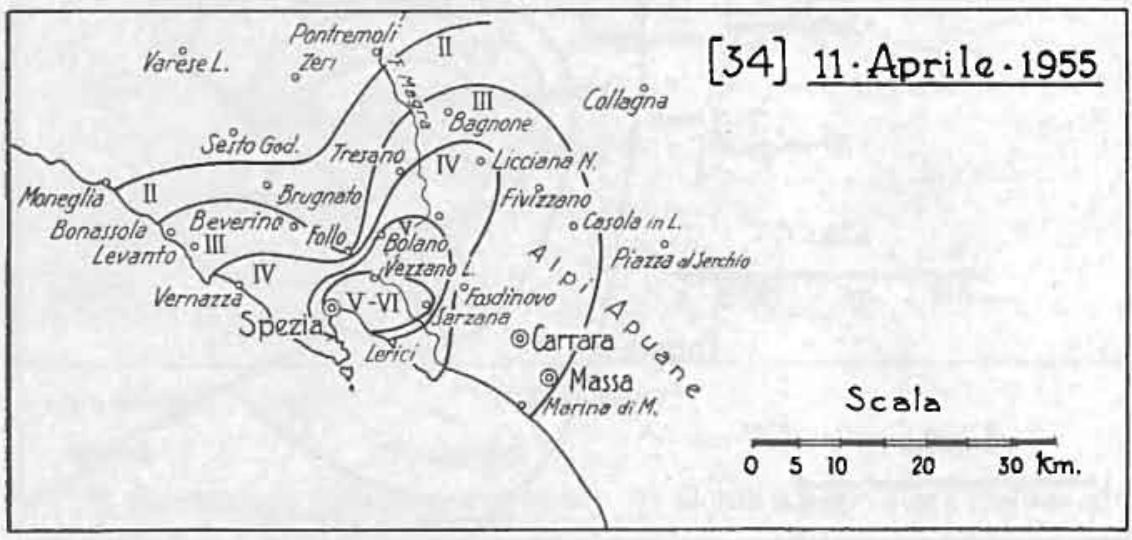

Fig. 22

maggiore intensità (V-VI grado) in una zona comprendente il capoluogo e i Comuni di Sarzana e di Vezzano Ligure. In questa zona, ove con molta probabilità si può ubicare l'epicentro, contemporaneamente alla scossa, avvertita come sussultoria e con una durata di circa $5^{\mathrm{s}}$, fu udito un forte boato sotterraneo. Nessun danno ma solo un forte spavento, specialmente in coloro che, trovandosi nei cinematografi o in locali chiusi in genere, avvertirono particolarmente la scossa.

Il movimento tellurico risultò forte ( $\mathrm{V}$ grado) nel comune di Bolano e sensibile (IV grado) a Vernazza, Licciana Nardi, Aulla, Lerici. La zona ove fu avvertito con intensità leggera è racchiusa da una isosista avente ai limiti i centri di Bonassola, Beverino, Follo, Bagnone, Casola in Lunigiana, Massa e Marina di Massa (v. fig. 22). 
Dopo le notevoli scosse telluriche avvenute nel Gargano durante il febbraio ed il marzo del 1955, altre repliche si ebbero in quella regione durante il successivo mese di aprile. Non abbiamo peró sicure notizie circa la loro intensità e le ore in eui si verificarono. Solo possiamo dire ch'esse furono tutte leggiere o mediocri e solo una, avvertita alle $10^{\text {th }}$ circa del 21 aprile raggiunse il IV-V grado Mercalli.

Un'altra replica, registrata negli Osservatorî di Taranto e di Roma, si ebbe nel mese di maggio, alle $06^{\mathrm{h}} 49^{\mathrm{m}}$ del giorno 5 . Fssa fu avvertita come sussultoria a $S$. Giovanni Rotondo e Monte S. Angelo e valutata, in queste località, di V grado Merralli. Per quanto riguarda la propagazione, ci risulta solo che la scossa fu avvertita di III grado a Manfredonia e di II a Rignano Garganien.

\section{12 maggio Stroppo-Brossasco (Cuneo)}

Alle $15^{\text {h }} 16^{\text {m }}$ del 12 maggio 1955 , una fortissima scossa di terremoto colpì la provincia di Cuneo in una zona compresa fra la Val di Maira e la Val Varaita.

Nel comune di Stroppo, in Valle Maira, la scossa, avvertita da tutti con grande spavento e fuga all'aperto, fu valutata di grado VII e causò crolli di fumaioli, caduta di ardesie dai tetti e lesioni leggiere, ma numerose, in diversi edifici anche di buona costruzione; di VI-VII grado risultó a Macra, pure in Valle Maira, e a Brossasco in Val Varaita; di VI, nei comuni di Pontechianale, Casteldelfino, Prazzo e Roccabruna, della provincia di Cuneo. In tutti i comuni predetti la scossa fu ondulatoria ed ebbe una durata di circa 4 secondi.

Con intensitì ove forte ove mediocre, il movimento tellurico fu sentito in molti comuni delle provincie di Cuneo e di Torino situati nella varie valli che solcano l'arco delle Alpi Piemontesi: di V grado nei comuni di Cervasca, Argentera, Marmora, Bellino, della provincia di Ouneo, e di Cavour, Pinerolo, San Germano Chisone e Torre Pellice, della provincia di Torino; di IV grado nei comuni di Borgo S. Dalmazzo, Roceavione, Morozzo, Mondovi, Caraglio, Busca, Monterosso Grana, Saluzzo, Sanfront, tutti della provincia di Cuneo, e di Angrogna, Campiglione Fenile, San Secondo di Pinerolo, Perosa Argentina e Piscina, della provincia di Torino. 


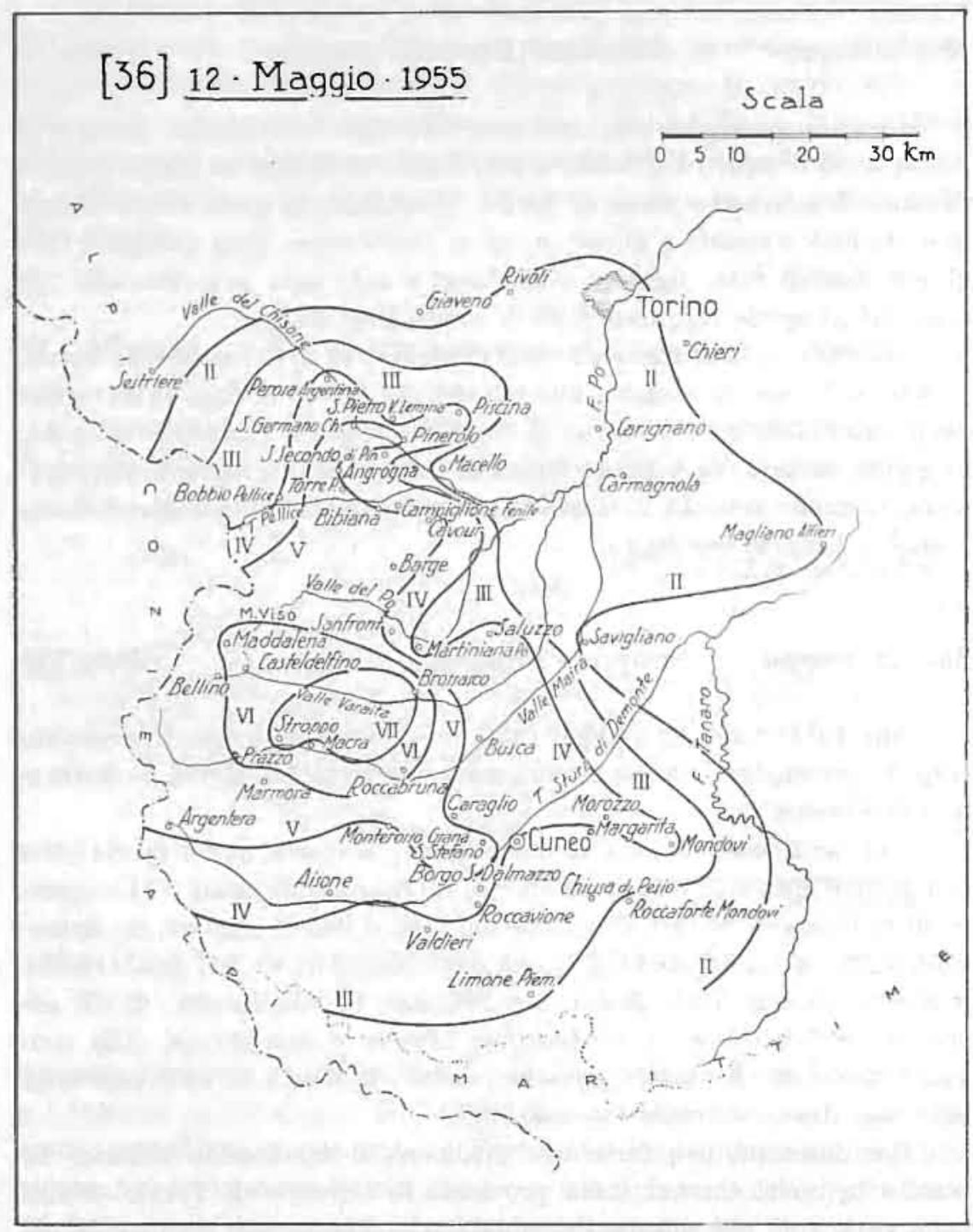

Fig. 23

La propagazione fu alquanto estesa ed irregolare. Non avendo notizie da oItre confine, ci limitiamo a dare, con la figura 23 , un'idea della distribuzione delle intensità nel solo territorio italiano.

Le coordinate epicentrali assegnate dal Bureau Central International Séismologique $\left(\varphi=44^{\circ} 30^{\prime} \mathrm{N}\right.$ e $\left.\lambda=07^{\circ} 18^{\prime} \mathrm{E}\right)$, in buon accordo con $\mathrm{i}$ 
rilevamenti macrosismici, individuano un punto situato in Val di Maira, and est di Stroppo e a sud-ovest di Brossasco.

Come ora della scossa all'epicentro, dalle registrazioni ottenute in molti Osservatori italiani, si ottenne $15^{\mathrm{h}} 15^{\mathrm{m}} 59^{\mathrm{s}}$.

Pochi giomi prima di questo terremoto, e precisamente intorno alle $09^{11} 30^{\mathrm{m}}$ del 9 maggio, erano state avvertite nel Pinerolese tre leggere scosse di earattere ondulatorio e della durata di pochi secondi. Xon si ebbe notizia di alcuna replica durante il mese di maggio.

37. 20 giugno

Prazzo (Cuneo)

Grado VTI

Un notevole terremoto, registrato in molti Osservatori italiani e stranieri e per il quale il B.C.I.S. assegna le stesse coordinate epicentrali date per il terremoto, precedentemente esaminato, del 12 maggio 1955, si verificó il mese successivo, alle $05^{\mathrm{h}} 48^{\mathrm{m}}$ del giorno 20 .

Il movimento tellurico, questa volta, raggiunse peró la sua maggiore intensità, valutata di VII grado, nel comune di Prazzo (Cuneo), mentre a Stroppo fu solo di V-VI grado. Molto forte (VT grado) riusci nei comuni di Marmora, Casteldelfino e Brossaseo. A Prazzo la scossa ebbe carattere sussultorio e fu accompagnata da un forte boato; pure come sussultoria fu avvertita a Marmora e a Brossasco; a Casteldelfino $\mathrm{fu}$ invece prevalentemente ondulatoria.

Nell'area isosismica forte risultano compresi i comuni di Pontechianale, Busca (Cuneo) e numerosi comuni della provincia di Torino, fra i quali Cavour, Pinerolo, Piscina, S. Germano Chisone, Torre Pellice e Villafranea Piemonte.

II moto sismico fu avvertito sensibilmente nei comuni di Angrogna, Bibiana, Buriasco, Campiglione Fenile, Cumiana, Garzigliana, Osasco, Perosa Argentina e S. Secondo di Pinerolo, tutti della provincia di Torino; di Saluzzo, Caraglio, Borgo S. Dalmazzo, Roccavione, Limone Piemonte, Roccabruna e Bellino, della provincia di Cuneo. Nelle città di Torino e di Cuneo la scossa fu solo leggermente avvertita.

Anche in questo terremoto, come in quello del 12 maggio, la propagazione macrosismica risultò assai estesa ed irregolare (v. fig. 24).

L'ora della scossa all'epicentro fu $05^{\mathrm{h}} 47^{\mathrm{m}} 30^{\mathrm{s}}$.

Non si ebbero repliche per tutto il mese di luglio. Il $1^{\circ}$ agosto, alle $9^{\mathrm{h}} 4^{\mathrm{m}}$ circa, una nuova scossa, di mediocre intensità, fu avvertita nel comune di Stroppo. 


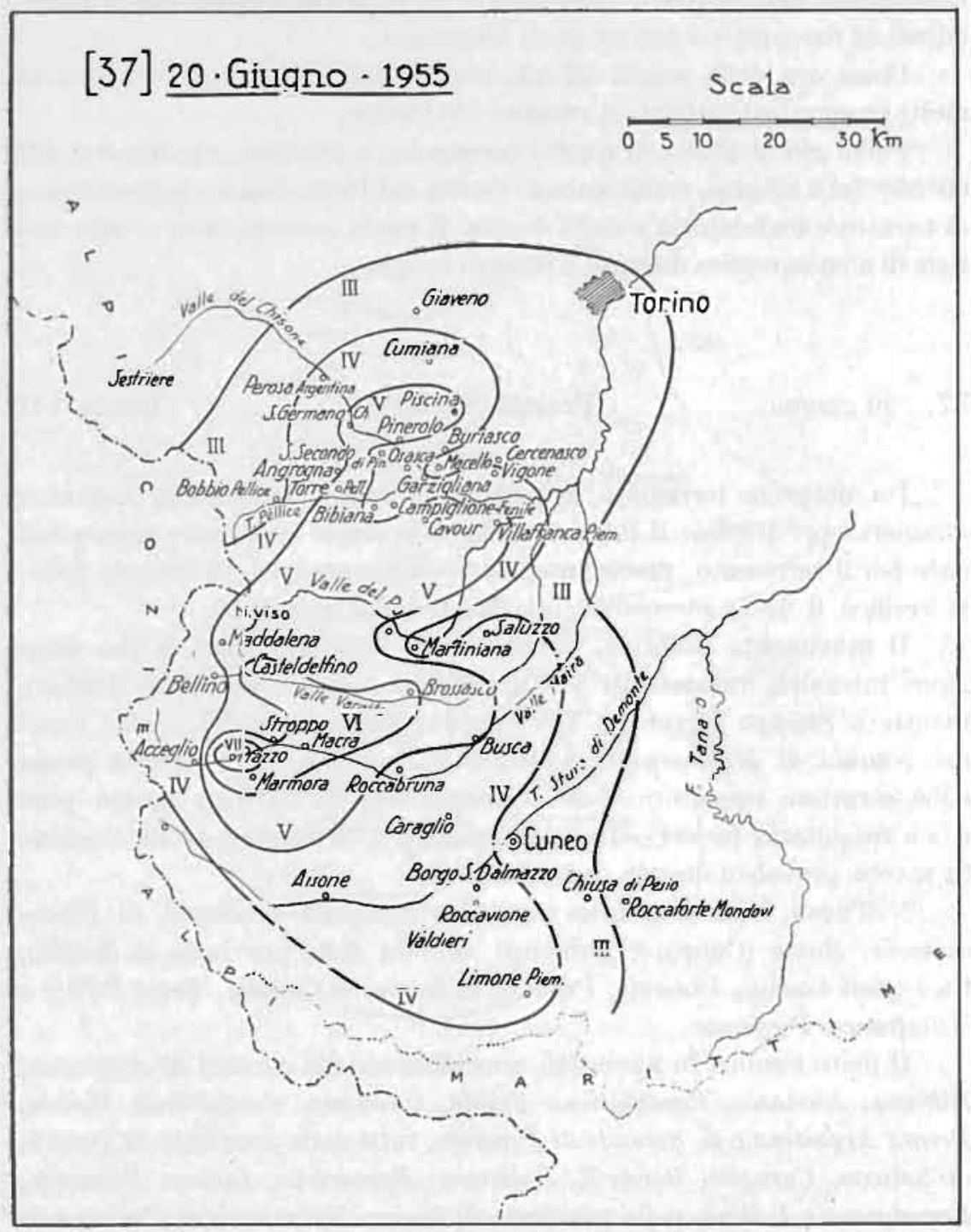

Fig. 24

38. 4 luglio

Una scossa molto forte di terremoto ebbe luogo alle $00^{\mathrm{h}} 55^{\mathrm{m}}$ curca del 4 luglio 1955 nella zona lucana ad ovest della montagna del Sirino. La località più colpita fu la cittadina di Lagonegro, ove la scossa, av- 
vertita da tutti con grande spavento, raggiunse il VI grado causando caduta di oggetti nelle case, screpolature negli intonaci e qualche leggera lesione negli edifici meno solidi. Tale scossa, il eui epicentro può ritenersi ubicato nei pressi del Monte Sirino, costituisce probabilmente una nuova manifestazione di un centro sismico già ben noto per fortissimi terremoti da esso originati nel passato (2 gennaio 1931 e 20 novembre 1936).

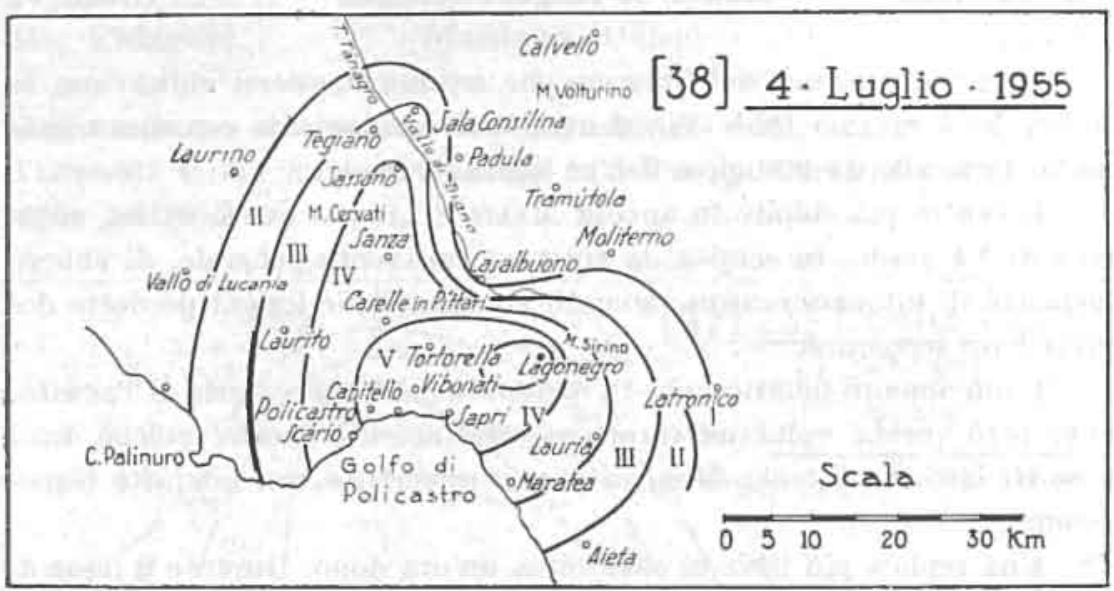

Fig. 25

Il moto tellurico si propagò con forte intensità ( $\mathrm{V}$ grado) soprattutto verso ovest, in provincia di Salerno: a Tortorella, Vibonati, Capitello, Policastro e Sapri fu avvertito dalla quasi totalità degli abitanti e produsse tremolio di grandi oggetti e caduta di calcinacci in alcune case. In tutte le località sopra citate fu accompagnato da rombi e risultò prevalentemente sussultorio. Decrebbe rapidamente d'intensità verso nord, tanto che a Casalbuono e a Moliterno passò del tutto inosservato (v. fig. 25).

La zona di mediocre intensità (IV grado) è limitata da una isosista che, partendo dalla costa tirrenica ad ovest di Scario, sale verso nord fin nel Vallo di Diano, include il comune di Sassano e, con una accentuata insenatura, torna al mare a sud di Maratea racchiudendo nel suo interno il comune di Lauria. Un andamento simile presenta la isosista di leggera intensità, comprendente, fra altri, i comuni di Sala Consilina e Laurito (Salerno).

Sotto forma microsismica, la scossa fu rilevata negli Osservatorî di Reggio C. e di Messina, i cui apparecchi registrarono i primi impulsi 
rispettivamente alle $00^{\mathrm{h}} 55^{\mathrm{m}} 33^{\mathrm{s}}, 3$ e $00^{\mathrm{h}} 55^{\mathrm{m}} 33^{\mathrm{s}}, 8$. Tracce di registrazione si ebbero anche negli Osservatorî di Roma e di Firenze.

Lievi repliche, ad ore però imprecisate, furono avvertite a Lagonegro durante la giornata del 5 luglio 1955 .

39. 12 luglio

Monte S. Angelo (Foggia)

Grado VI

Il periodo sismico del Gargano che sembrava essersi chiuso con la scossa del 5 maggio 1955 (35), si manifestò nuovamente con una scossa molto forte alle $05^{\mathrm{h}} 02^{\mathrm{m}}$ circa del 12 luglio successivo.

Il centro più colpito fu ancora Monte $S$. Angelo ove la scossa, valutata di VI grado, fu sentita da tutti con spavento generale. Si ebbero distacchi di intonaci e aggravamento notevole delle lesioni prodotte dai precedenti terremoti.

Il movimento tellurico, che fu registrato nell'Osservatorio di Taranto, ebbe però questa volta un carattere strettamente locale, poichè, fra i i centri circostanti, solo Manfredonia lo avverti, e con intensità leggerissima.

Una replica più lieve si ebbe circa un'ora dopo. Durante il mese di luglio 1955 non furono avvertiti altri movimenti tellurici. Ricominciarono nell'agosto successivo con una scossa avvenuta il giorno 4 alle $21^{\mathrm{h}} 15^{\mathrm{m}}$ ed avvertita di IV-V grado a Monte S. Angelo. Dopo di essa, durante il mese di agosto e sempre a Monte $S$. Angelo, si ebbero una scossa di IV grado alle $07^{\mathrm{h}} 07^{\mathrm{m}}$ del giorno 7 , una di IV-V grado alle $02^{\mathrm{h}} 47^{\mathrm{m}}$ del 19 ed una di III grado alle $02^{\mathrm{h}} 30^{\mathrm{m}}$ del 22 . Due scosse, l'una di IV e l'altra di III grado, si verificarono rispettivamente $1^{\text {' }} 8$ settembre alle $06^{\text {h }} 19^{\mathrm{m}} \mathrm{e}$ l'11 ottobre alle $12^{\mathrm{h}} 30^{\mathrm{m}}$. Il periodo sismico si chiuse il 2 novembre 1955 con tre scosse d'intensità leggera, avvenute, rispettivamente, alle $05^{\mathrm{h}} 50^{\mathrm{m}}$, alle $09^{\mathrm{h}} 25^{\mathrm{m}}$ e alle $21^{\mathrm{h}} 15^{\mathrm{m}}$.

40. 22 luglio San Demetrio Corone (Cosenza)

Grado V

Con una scossa avvertita di IV grado a San Cosmo Albanese (Cosenza), ebbe inizio, alle $13^{\mathrm{h}} 45^{\mathrm{m}}$ del 22 luglio 1955 , un notevole periodo sismico che, per diversi giorni, tenne in vivissima ansia le popolazioni di numerosi paesi situati nella zona calabra compresa tra la Presila, il fiume Crati e la costa jonica. Altri movimenti tellurici di leggera intensità seguirono durante il pomeriggio del 22 luglio finchè, alle $22^{\mathrm{h}} 00^{\mathrm{m}}$, preceduta da un 
rombo sotterraneo, si verifico una forte scossa che raggiunse a San Demetrio Corone (Cosenza) il V grado Mercalli e fu avvertita di IV grado a San Lorenzo del Vallo e a Vaccarizzo Albanese (Cosenza).

Segui una sensibile replica alle $24^{\text {th }}$ e poi numerose altre durante la giornata del 23 luglio; di esse si dirà fra poco, al n, 42.

41. 23 luglio

Maniago (Udine)

Grado VI

Una numerosa serie di movimenti tellurici, culminata in una scossa d'intensità molto forte, ebbe luogo nelle Prealpi Carniche dal 21 al 31 luglio del 1955.

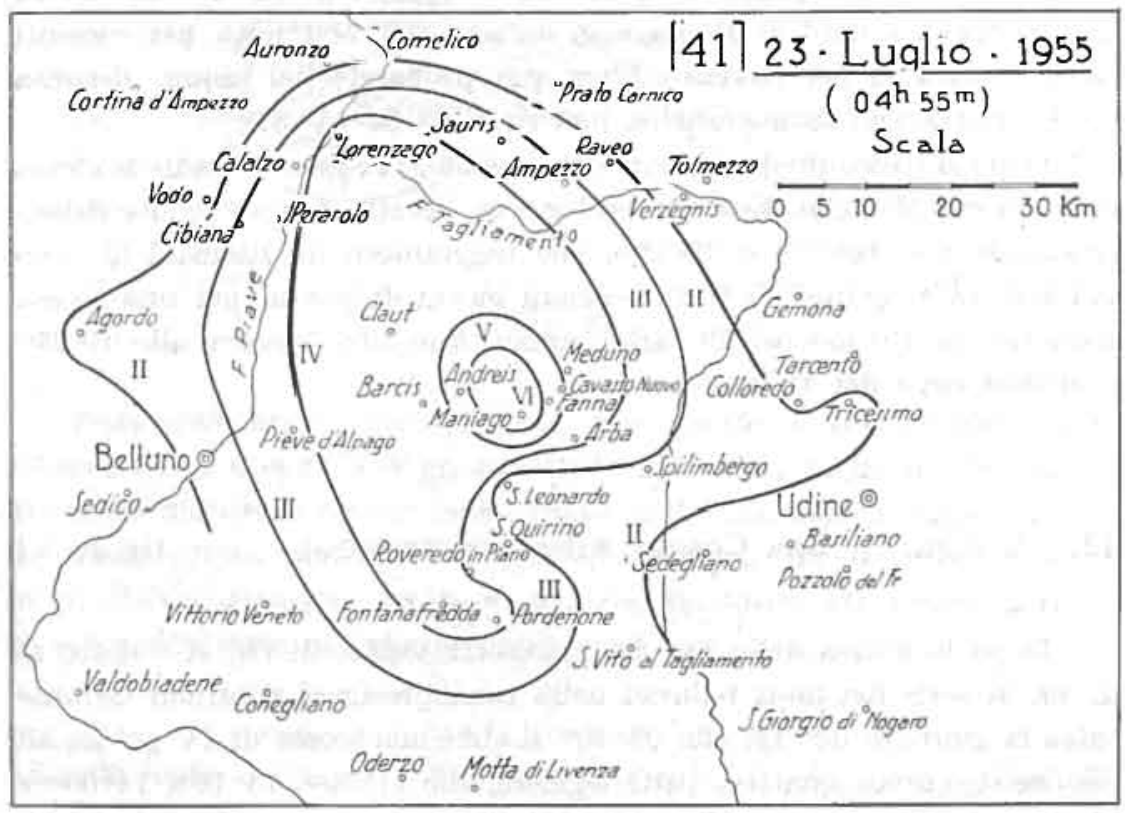

Fig. 26

II periodo sismico s'iniziò alle $22^{\mathrm{h}} 32^{\mathrm{m}}$ del giorno 21 con una scossa avvertita di IV grado a Maniago, alla quale ne segui un'altra, leggera, pochi minuti più tardi. II giorno successivo, alle $9^{\mathrm{t}} 50^{\mathrm{m}}$, si ebbe una scossa di IV-V grado e il giorno 23 , alle $04^{\mathrm{h}} 55^{\mathrm{m}}$, si verifico la scossa principale, che nel comune di Maniago, ove l'intensità fu valutata di VI grado, generò vivissimo spavento e causò leggere lesioni in numerose case di 
difettosa costruzione. In detta località questa scossa fu accompagnata da un forte rombo sotterraneo, ebbe carattere ondulatorio ed una durata brevissima (2-3 sec.).

Dalla cartina delle isosiste (fig. 26) si rileva che il movimento si mantenne intenso soprattutto in direzione di Lorenzago, cioè verso NNW,e di Pordenone, cioè verso sud. Lungo quest'ultima direzione si ebbe però l'anomalia che il movimento passó quasi inosservato a S. Leonardo, distante meno di una decina di chilometri da Maniago, mentre fu leggermente avvertito nei comuni di San Quirino e Roveredo in Piano e sensibilmente in quelli di Fontanafredda e Pordenone.

In base alle notizie macrosismiche pervenuteci e ai dati ottenuti dalle registrazioni di Salò e di Bologna, pensiamo che l'epicentro possa ritenersi ubicato nei pressi del punto di coordinate $46^{\circ} 12^{\prime} \mathrm{N}$ e $12^{\circ} 43^{\prime} \mathrm{E}$. Esso si trova a nord di Maniago in un'area già ben nota per violenti sismi verificatisi nel passato. L'ora più probabile del sisma, desunta dalle registrazioni sismografiche, può ritenersi $04^{\mathrm{h}} 54^{\mathrm{m}} 31^{\mathrm{s}}$.

Dopo la scossa predetta, numerose rephche si ebbero durante la stessa giornata del 23 luglio. Esse furono leggiere, eccetto due, avvenute rispettivamente alle $10^{\mathrm{h}} 25^{\mathrm{m}}$ e $20^{\mathrm{h}} 29^{\mathrm{m}}$, che raggiunsero un'intensità di poco inferiore al $\mathrm{V}$ grado. Vi furono alcuni giorni di pausa, poi una scossa sensibile alle $19^{\mathrm{h}} 35^{\mathrm{m}}$ del 30 luglio, quindi due altre leggiere alle $01^{\mathrm{h}} 25^{\mathrm{m}}$ e $04^{\mathrm{h}} 30^{\mathrm{m}}$ circa del 31 luglio.

42. 23 Iuglio San Cosmo Albanese (Cosenza)

Grado VI

Dopo la scossa delle $24^{\text {h }}$ del giorno 22 luglio, di cui si è detto al n. 40, Ia serie dei moti tellurici nella zona presilana continuò durante tutta la giornata del 23: alle $03^{\mathrm{h}} 40^{\mathrm{m}}$ si ebbe una scossa di IV grado; ad essa ne seguirono quattro, tutte leggiere, alle $11^{\mathrm{h}} 50^{\mathrm{m}}, 13^{\mathrm{h}} 10^{\mathrm{m}}, 14^{\mathrm{h}} 00^{\mathrm{m}} \mathrm{e}$ $21^{\mathrm{h}} 50^{\mathrm{m}}$ rispettivamente. Alle $22^{\mathrm{h}} 02^{\mathrm{m}}$, infine, sopraggiunse la scossa principale del periodo sismico, che provocò grande panico nei centri più colpiti inducendo le popolazioni ad abbandonare le abitazioni e a rifugiarsi nelle vicine campagne sotto attendamenti improvvisati alla meglio. Questa scossa, registrata negli Osservatori di Messina e di Taranto, raggiunse Ia sua maggiore intensità (VI grado) a San Cosmo Albanese e a Vaccarizzo Albanese. In queste due località, fra le quali molto probabilmente fu l'epicentro $\left(\varphi=39^{\circ} 35^{\prime}\right.$ e $\left.\lambda-16^{\circ} 25^{\prime}\right)$, il moto sismico fu preceduto da rombi intensi e causó leggiere fenditure in molte case. 
La scossa fu forte a San Giorgio Albanese e a San Demetrio Corone, mediocre a Bisignano, Santa Sofia d'Epiro, Corigliano Calabro ed Acri. La figura 27 ne indica le isosiste fino a quella di grado $\mathrm{rV}$, non avendo avuto a nostra disposizione notizie macrosismiche sufficienti per poter tracciare anche quelle di grado inferiore.

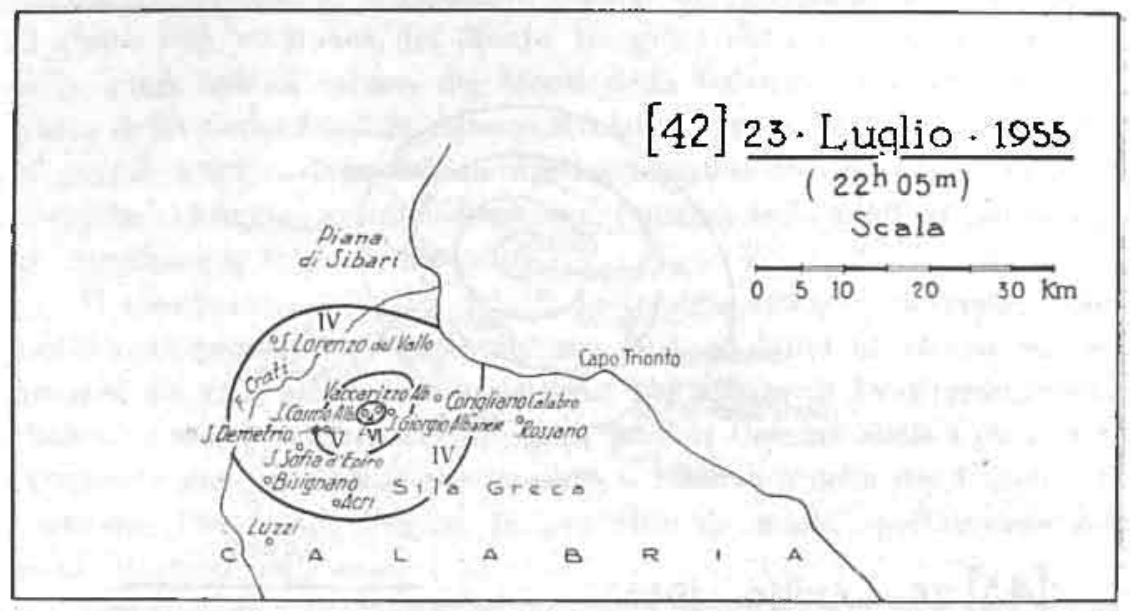

Fig. 27

Seguirono due leggiere repliche, alle $22^{\mathrm{h}} 15^{\mathrm{m}}$ e alle $22^{\mathrm{h}} 30^{\mathrm{m}}$ rispettivamente, ed una di IV-V grado alle $23^{\mathrm{h}} 17^{\mathrm{m}}$. Il 24 luglio furono ancora avvertite numerose scosse: esse furono tutte d'intensità leggera o leggerissima eccetto una, di IV-V grado, avvenuta alle $00^{\mathrm{h}} 05^{\mathrm{m}}$, la quale, in $S$. Cosmo Albanese, accentuó qualche fenditura già prodotta dalla scossa molto forte del giorno precedente.

43. 26 Iuglio

Colonna (Roma)

Grado $V$

Il 26 luglio 1955 , alle $15^{\mathrm{h}} 13^{\mathrm{m}}$ circa, venne avvertita nel Comune di Colonna, situato nel versante nord dell'apparato vuleanico dei Colli Albani, un movimento tellurico che fu valutato d'intensità parì al $\mathrm{V}$ grado della Scala Mercalli. Poichè nelle località viciniori la scossa risultò ovunque meno intensa, riteniamo che l'epicentro possa essere ubicato in prossimità di Colonna e che la scossa stessa sia una nuova manifestazione di un centro sisuico situato appunto nei pressi di detta località e già noto per altre scosse da esso originate nel passato. 
La propagazione del moto tellurico, come in generale accade per i terremoti di natura vulcanica, anche forti, fu assai limitata: a Frascati, distante da Colonna poco più di sei chilometri in linea d'aria, la scossa venne infatti solo leggermente avvertita; leggerissima fu a Grottaferrata, Marino e Rocea di Papa; del tutto inavvertita passò a Palestrina e a Labico.

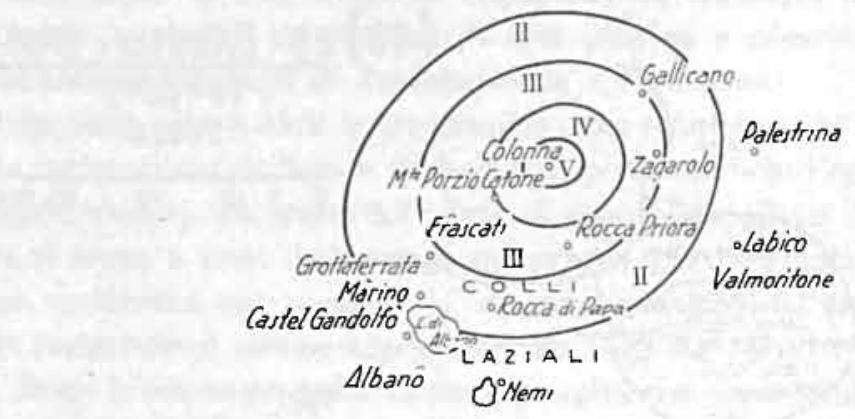

[43] 26- Luglio 1955

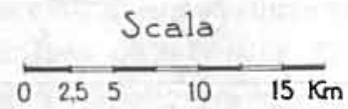

Fig. 28

La figura 28 serve a dare un'idea dell'estensione dell'area ove il movimento fu percepito macrosismicamente. In realtà le isosiste non debbono aver avuto un andamento cosi regolare poichè, nella regione dei Colli Laziali, la varietà delle condizioni litologiche e tettoniche genera sempre una certa irregolarità nella distribuzione delle intensità; irregolarità che però, in questo caso, non ci è stato possibile mettere in evidenza, data l'esiguità della scossa e la scarsezza delle notizie macrosismiche che abbiamo potuto raccogliere.

Il sisma fu registrato nell'Osservatorio di Roma con inizio alle $15^{\mathrm{h}} 13^{\mathrm{m}} 31^{\mathrm{s}}, 6$.

44. 25 settembre

Prato (Firenze)

Grado V

La notte del 25 settembre 1955 , alle $01^{\mathrm{h}} 49^{\mathrm{m}}$ circa, in molte località delle province di Firenze e di Pistoia, le popolazioni furono destate da 
un sensibile movimento tellurico risultato particolarmente intenso nel Pratese.

In base alle registrazioni sismografiche ottenute negli Osservatorî di S. Domenico in Prato e Ximeniano di Firenze, sembra che l'epicentro possa essere collocato nei pressi del punto di coordinate geografiche $43^{\circ} 56^{\prime} \mathrm{N}, 11^{\circ} 09^{\prime} \mathrm{E}$ e cioè nel Bacino del Bisenzio, a settentrione di Prato e in vicinanza del Monte Maggiore che è la cima più elevata della lunga catena carsica dei Monti della Calvana. Probabilmente si tratta dello stesso focolare sismico rivelatosi con la fortissima seossa del 27 giugno 1899 e, dopo tale data, manifestatosi ancora una volta il 19 novembre 1940 con tre modeste scosse, l'ultima delle quali, la più intensa, raggiunse il $\mathrm{V}$ grado Mercalli.

Il movimento tellurico del 25 settembre 1955 , di $\mathrm{V}$ grado nelle località più prossime all'epicentro, non provocò danni di alcuna specie, ma solo un vivo allarme nelle persone. Fu sentito di IV-V grado nella città di Prato e con uguale intensità in vari Comuni della Conca dell'Ombrone pistoiese, come per esempio a Tizzana e nella sua frazione di Quarrata (Pistoia). A Firenze fu avvertito da molti, specialmente nei piani superiori delle case.

L'ora esatta della scossa all'epicentro, dedotta dalle registrazioni di Prato e di Firenze, è $01^{\mathrm{h}} 48^{\mathrm{m}} 40^{\mathrm{s}}$.

\section{29 settembre Marzano Appio (Caserta) Grado V}

Ad est dei Monti Aurunci, separata da essi dalla valle del medio Garigliano, si eleva la Montagna di Roccamonfina, grande vulcano a recinto, oggi inattivo, dal cui ampio cratere s'innalza, fino ad oltre mille metri sul livello del mare, il cono centrale di Monte S. Croce. La zona su cui la montagna vulcanica estende le sue vaste pendici ammantate di rigogliosi castagneti e popolate di paesi e di antiche cittadine è una zona di lieve sismicità e le scosse telluriche che in essa si ebbero nel passato, anche se intense, come quella fortissima del febbraio 1728 e quella molto forte del 17 dicembre 1935, rimasero sempre limitate ad aree assai ristrette.

Carattere locale ebbe anche la scossa avvenuta alle $02^{\mathrm{h}} 10^{\mathrm{m}}$ eirea del 29 settembre 1955 , la quale raggiunse la sua maggiore intensità (V grado) nel comune di Marzano Appio, sul versante NE del vulcano. $\Lambda$ Roccamonfina, la cittadina che dà nome al vuleano, situata nel lato $\mathrm{E}$ 


\section{[45] 29. Settembre $\cdot 1955$}

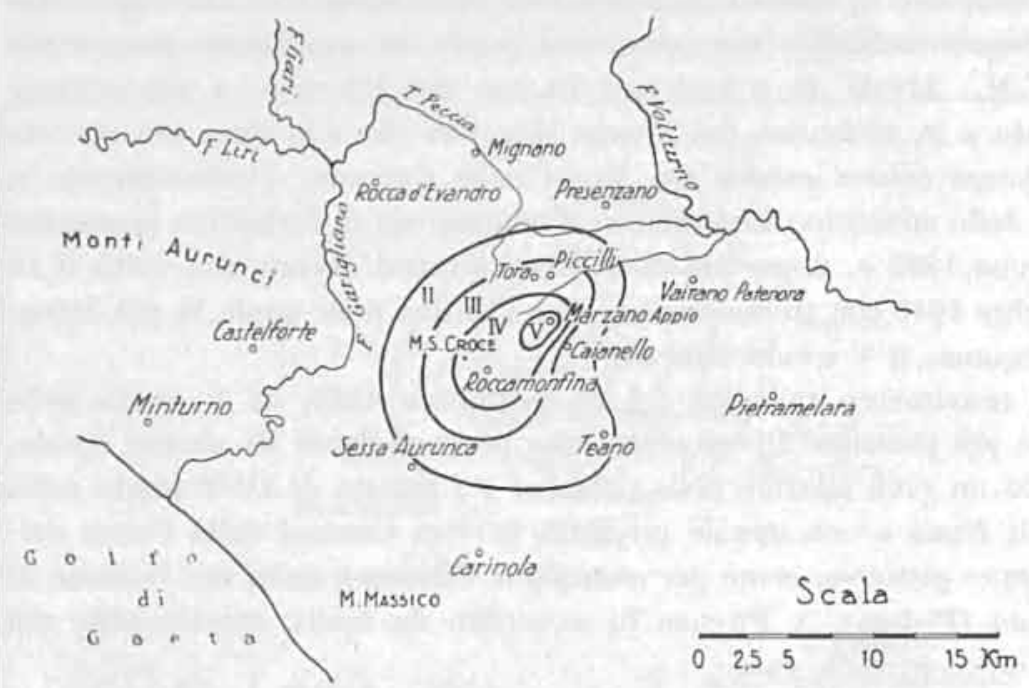

Fig. 29

del grande cratere, il movimento tellurico fu avvertito con sensibile intensità e, con intensità leggera, nel Comune di Tora e Piccilli (fig. 29).

Non si ebbero repliche.

46. 9 ottobre

Stroppo (Cuneo)

Grado V

Una scossa ondulatoria $\mathrm{E}$, di intensità abbastanza forte (V grado) ma di brevissima durata (2 sec.), fu sentita nel comune di Stroppo alle $9^{\mathrm{h}} 10^{\mathrm{m}}$ del 9 ottobre 1955 . La propagazione macrosismica dovette essere assai limitata, poichè nessuno dei Comuni vicini segnalò di aver avvertito la scossa.

Una replica di III grado si ebbe il mese successivo, alle $15^{\text {h }}$ circa del giorno 3 .

47. 13 dicembre

Carrara

Grado V

Il 13 dicembre 1955 , alle $18^{\mathrm{h}} 04^{\mathrm{m}}$ circa, una sensibile scossa tellurica fu avvertita in numerose località delle province di La Spezia, Massa- 
Carrara e Lucca. Le coordinate epicentrali del sisma, calcolate dall'I.N.G. in base alle registrazioni ottenute negli Osservatori dell'Italia settentrionale, risultarono $44^{\circ} 07^{\prime} \mathrm{N}$ e $10^{\circ} 09^{\prime} \mathrm{E}$. Il punto da esse individuato si trova nelle Alpi Apuane, a nord est di Carrara ed è distante pochi chilometri dal Pizzo d'Uccello, l'alto monte apuano dai cui pressi ebbe origine il rovinosissimo terremoto dell'aprile 1837.

La scossa del 13 dicembre 1955 si propagò su una superficie abbastanza estesa, ma purtroppo le notizie macrosismiche a noi pervenute non sono sufficienti per poter tracciare le isosiste. Nel comune di Carrara il movimento tellurico fu sentito fortemente ( $\mathrm{V}$ grado); non provocò alcun danno, ma il forte boato da cui subito dopo fu seguito causò un fortissimo spavento in tutte le persone che immediatamente abbandonarono le abitazioni ed i pubblici locali per portarsi nelle piazze e nelle strade. Con sensibile intensità fu avvertito nella città di Massa, in diverse località della Valle del Fiume Magra, come Tresana e Barbarasco, e nella città di La Spezia.

Anche la provincia di Lucea fu interessata dal movimento tellurico e in modo particolarmente sensibile il Camaiorese. A Camaiore la scossa fu sentita di V grado e causò vivissimo allarme nella popolazione. A Viareggio, invece, il fenomeno risultò leggerissimo e fu avvertito da poche persone. Nella città di Lucca fu sentito di III-IV grado.

L'ora della scossa all'epicentro, dedotta dalle registrazioni sismografiche, risultò $18^{\mathrm{h}} 04^{\mathrm{m}} 07^{\mathrm{s}}$. 


\section{Anno 1956}

48. 9 gennaio

Grassano (Matera)

Grado VII

Il 9 gennaio 1956 , alle $01^{\mathrm{h}} 14^{\mathrm{m}}$ circa, una notevole scossa di terremoto, propagatasi macrosismicamente entro una zona molto vasta, ebbe luogo nella regione lucana, in provincia di Matera.

La maggiore intensità (grado VII) si verificò in una zona compresa fra le valli dei fiumi Bradano e Basento. L'epicentro fu nelle vicinanze di Grassano (Matera), all'incirca nel punto di coordinate geografiche $40^{\circ} 37^{\prime} \mathrm{N}$ e $16^{\circ} 18^{\prime} \mathrm{E}$. Quest'epicentro compare forse per la prima volta nella cronistoria dei terremoti della regione lucana; non abbiamo potuto infatti trovare alcuna notizia di movimenti tellurici anteriori, di una certa importanza, ai quali possa essere attribuito un epicentro interno all'area megasismica predetta, la quale, nella carta sismica del Baratta, figura in una zona giudicata come asismica.

La scossa del 9 gennaio 1956 cagionò danni notevoli all'abitato di Grassano: circa 200 case, sulle 3000 di cui esso è costituito, rimasero lesionate ed alcune cosi gravemente da dover essere sgombrate per timore di crolli; un edificio, forse in non buone condizioni di stabilità, crollò parzialmente e due persone rimasero ferite. Fortunatamente non si ebbero vittime.

La zona isosismica molto forte (fig. 30), a forma di ovale con l'asse maggiore in direzione EW ed estendentesi da Calciano, nella Valle del Basento, a Montescaglioso nella Valle del Bradano, rachiude nel suo interno, oltre a questi due Comuni, anche quelli di Grottole, Miglionico e Salandra. In tutte le citate località, ove la scossa, preceduta da un forte boato sotterraneo, ebbe carattere sussultorio ed una duranta di 4-5 secondi, si verificarono fenditure più o meno sensibili in diverse case ed in qualche edificio pubblico,

Nella città di Matera il movimento tellurico fu di forte intensità. Non si ebbero danni, ma vivissimo fu anche qui l'allarme della popolazione che, incurante del freddo intenso, si riversò nelle strade e nelle piazze, trascorrendo all'aperto gran parte della notte. La isosista di grado V, di forma quasi circolare, comprende, oltre Matera, anche i comuni di Irsina, Ferrandina, Pisticci, Pomarico, Bernalda (tutti in provincia di Matera) e si estende in provincia di Taranto includendo il comune di Ginosa. 
La propagazione macrosismica fu, come si è detto, notevolmente ampia. Oltre alle provincie di Matera e di Potenza, anche quelle di Taranto, Bari e Foggia furono interessate dal movimento tellurico, il quale, in quasi tutta la zona delle Murge, fu avvertito con sensibile intensità e, sulla costa adriatica da Trani a Monopoli, con intensità ove di III ove di II grado.

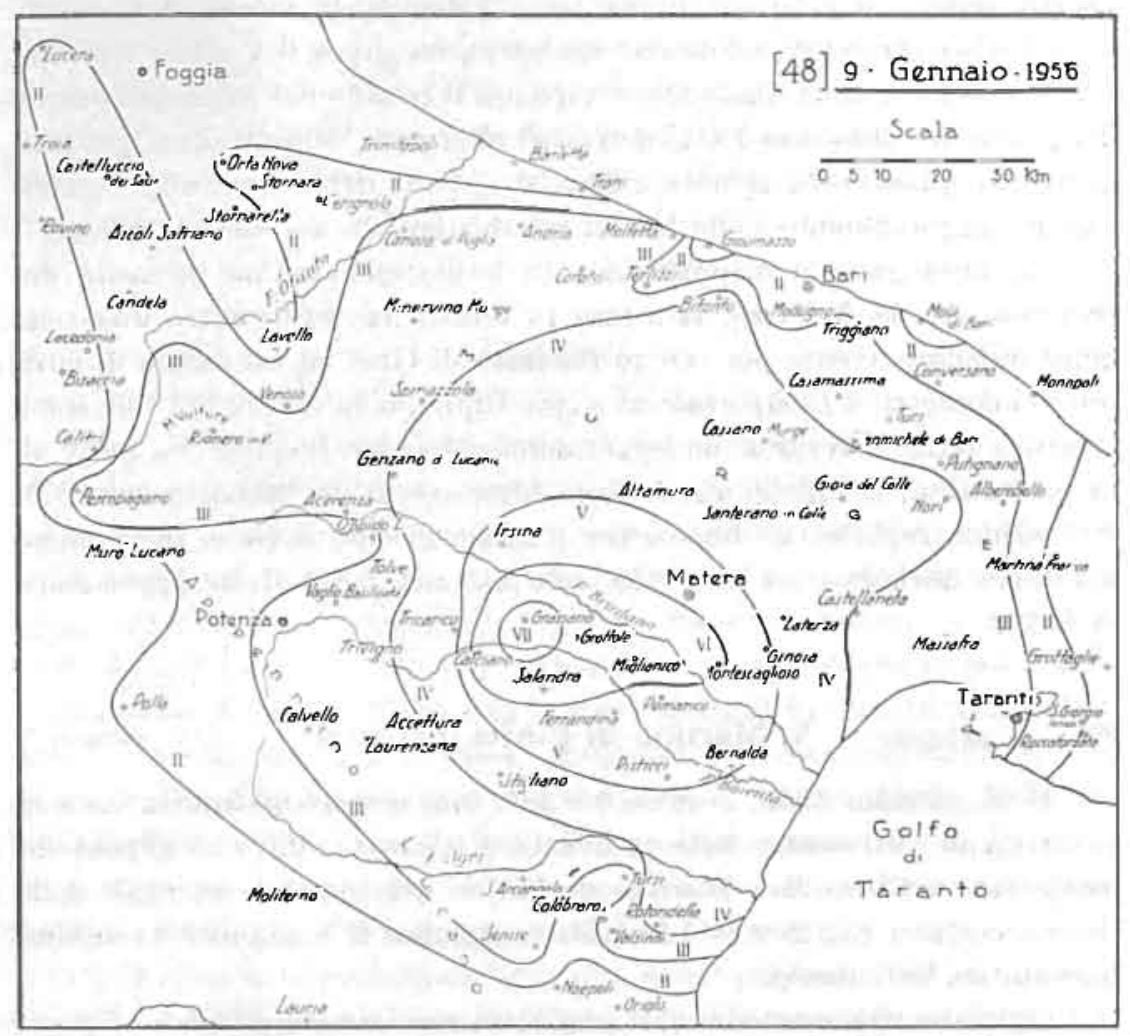

Fig. 30

La figura 30 ei esime dal dare il lungo elenco delle località ove la scossa fu percepita. Come da essa si rileva, Ia zona megasismica risulta assai eccentrica rispetto alle zone isosismiche di grado inferiore e la distribuzione delle intensità presenta varie anomalie: per esempio a Lavello (Potenza) il movimento tellurico passò completamente inosservato mentre fu avvertito a Ortanova e a Lucera, assai più distanti dalla zona epicentrale.

Fatto piuttosto raro in sismi di elevata intensità, questo terremoto non fu preceduto da alcuna scossa premonitoria nè fu seguito da repliche. 
49. 15 gennaio

Grisì (Palermo)

Grado V

Alle $20^{\mathrm{h}} 19^{\mathrm{m}}$ circa del 15 gennaio 1956 , dopo quasi un anno dal periodo sismico di cui si è parlato al $\mathrm{n}$. 27 , un altro movimento tellurico di carattere strettamente locale ebbe luogo nel territorio di Grisi. In questo paesino e nelle sue immediate vicinanze, la scossa, nettamente sussultoria e preceduta da un intenso boato, raggiunse il $\mathrm{V}$ grado Mercalli. La popolazione, nella quale ancor vivo era il ricordo dei fenomeni sismici del novembre-dicembre 1954, si riversò allarmata nelle strade e trascorse all'aperto quasi tutta la notte. Non si ebbero danni sensibili: si notò solo un aggravamento delle lesioni già esistenti in vecchie abitazioni.

La propagazione macrosismica fu limitatissima. Con intensità decrescente con la distanza, la scossa fu infatti avvertita entro una zona quasi circolare avente per centro l'abitato di Grisì ed un raggio di circa sette chilometri: a Camporeale ed a San Cipirello, ai margini di tale zona, la scossa venne percepita con leggerissima intensità. L'epicentro, con molta probabilità, fu quello stesso della forte scossa del 20 novembre 1954 .

Alcune repliche si ebbero, per qualche giorno, dopo il 15 gennaio; ma tutte lievissime ed avvertite solo da una parte della popolazione di Grisi.

50. 27 gennaio

S. Martino di Finita (Cosenza)

Grado V

Il 27 gennaio 1956 , a circa $06^{\mathrm{h}} 20^{\mathrm{m}}$, una scossa abbastanza forte fu avvertita in vari centri della provincia di Cosenza. Più che altrove intensa essa risultò a San Martino di Finita, nel versante orientale della Catena costiera calabra: in tale paese raggiunse il $\mathrm{V}$ grado e fu accompagnata da forti rombi.

Con intensità mediocre (IV grado) fu avvertita a Montalto Uffugo, in vari centri della regione silana, come Bisignano, Luzzi ed Acri e, sulla fascia costiera tirrenica, da Fuscaldo a Belvedere Marittimo. A Cosenza fu lievissima.

Tracce di registrazioni si ebbero negli Osservatorî di Reggio Calavria e di Taranto.

50. 31 gennaio

Istria

Grado VII-VIII

Questa violenta scossa di terremoto ebbe il suo epicentro fuori del confine italiano ma fu avvertita fortemente a Trieste ed in altre località della Venezia Giulia. Il sisma, le cui coordinate epicentrali $\left(45^{\circ} 30^{\prime} \mathrm{N}\right.$ 
e $14^{\circ} 30^{\prime} \mathrm{E}$, secondo il B.C.I.S.) individuano un punto situato una decina di chilometri a sud del Monte Nevoso, colpì in special modo Villa del Nevoso, ove più della metà delle case rimasero seriamente danneggiate. A Trieste e Muggia fu avvertito di V grado, a San Dorligo della Valle e ad Aurisina di IV.

Il movimento sismico si propagò anche nelle province di Gorizia e di Udine e fu avvertito con sensibile intensità a Grado e a Ruda.

L'ora origine, secondo il B.C.I.S., fu $03^{\mathrm{h}} 25^{\mathrm{m}} 32^{\mathrm{s}}$.

Il terremoto ebbe numerose repliche: la più notevole, avvenuta alle $14^{\mathrm{h}} 42^{\mathrm{m}}$ circa del 3 febbraio, fu avvertita a Trieste di III-IV grado e di III a San Dorligo della Valle.

Alle $16^{\mathrm{h}} 11^{\mathrm{m}}$ circa del $1^{\circ}$ febbraio 1956 , una scossa di terremoto venne avvertita in molte località della Calabria, delle Puglie e, lievemente, anche della Sicilia. Più forte che altrove essa si manifestò a Cosenza e nella zona circostante, ove fu avvertita da tutta la popolazione e raggiunse un'intensità valutata di $\mathrm{V}$ grado. Sensibile risultò su tutta la parte tirrenica della Calabria da Diamante a Vibo Valentia e, più a sud, in una zona intorno a Palmi. Pure sensibilmente fu avvertita a Catanzaro e nei comuni di San Floro, Magisano, Taverna, Serrastretta, Scigliano, e Parenti. Leggera fu invece a Saracena, Firmo, Altilia, Cellara e Bianchi. Sulla fascia costiera jonica passò quasi inosservata, ad eccezione delle zone circostanti a Corigliano Calabro ed a Rocca Imperiale ove fu avvertita, rispettivamente, di IV e di III grado.

Nelle Puglie, e precisamente in una zona comprendente i comuni di Castellaneta, Palagiano, Massafra e Crispiano, la scossa fu avvertita con intensità di IV grado.

La vastità e l'irregolarità della propagazione macrosismica di questo movimento tellurico (fig. 31) ne attestano già la notevole profondità ipocentrale. In un suo studio dedicato ai terremoti profondi del Mare Tirreno $\left({ }^{5}\right)$, E. Peterschmitt assegna alle coordinate ipocentrali del sisma i valori seguenti: $\varphi=39^{\circ}, 2 \mathrm{~N} ; \lambda=15^{\circ}, 7 \mathrm{E} ; \mathrm{h}=215 \mathrm{Km}$.

Questo sisma si inserisce pertanto nella serie dei terremoti di origine profonda caratteristici della regione del Basso Tirreno. Di altri due terremoti tirrenici, avvenuti rispettivamente il 23 novembre 1954 ed il 17 febbraio 1955, non ci siamo precedentemente occupati per non aver essi dato luogo in superficie ad apprezzabili manifestazioni macrosismiche. 


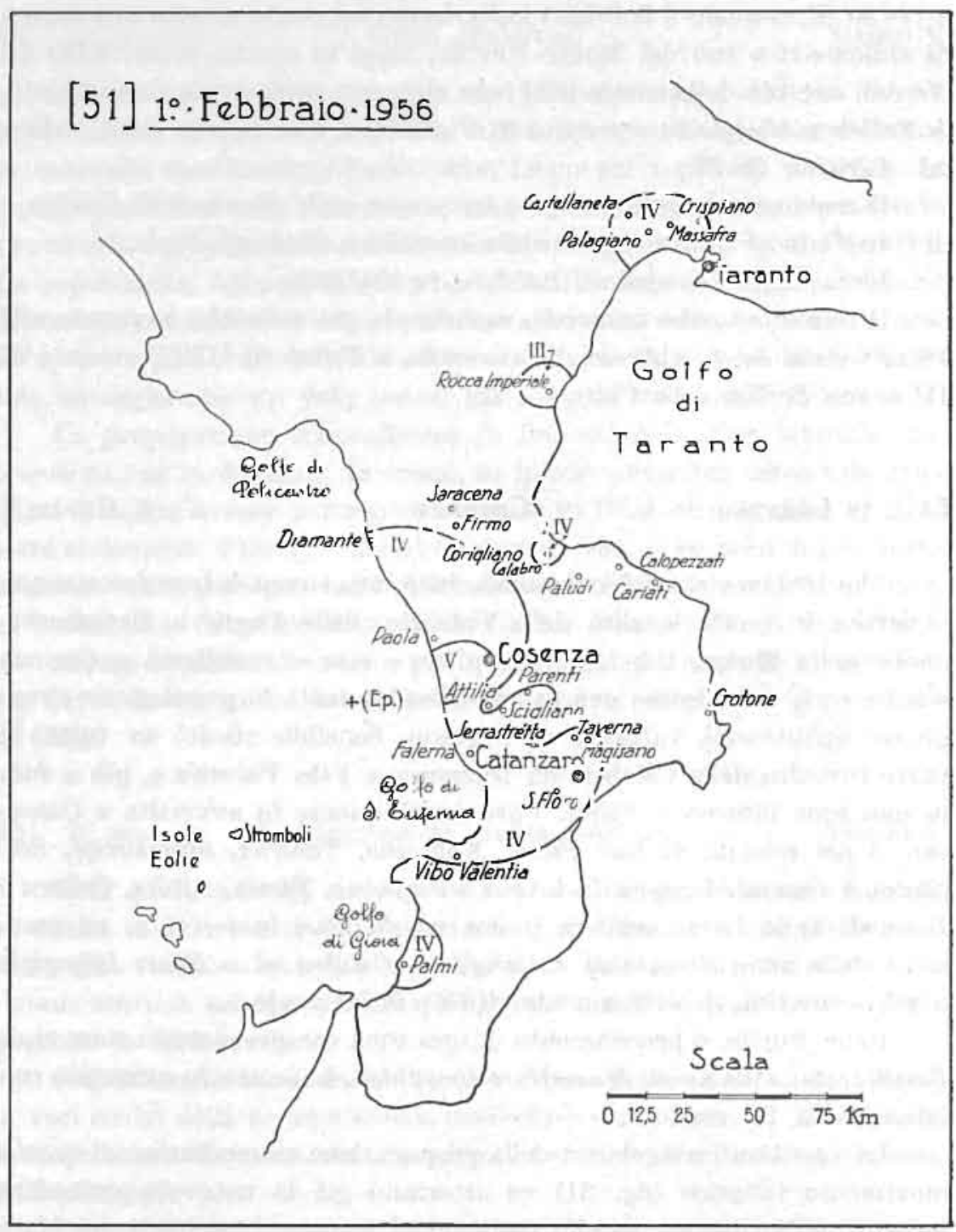

Fig. 31

Ci limiteremo qui a dire che le coordinate spazio-temporali del primo, determinate da A. Girlanda in uno studio ad esso dedicato $\left.{ }^{8}\right)$, sono: $\varphi=38^{\circ} 33^{\prime} \mathrm{N}, \lambda=15^{\circ} 01^{\prime} \mathrm{E}, \mathrm{h}=239 \mathrm{Km}, \mathrm{H}=13^{\mathrm{h}} 00^{\mathrm{m}} 05^{\mathrm{s}}, 5$; e che quelle del secondo, date da Peterschmitt nel lavoro avanti citato, risultano: $\varphi=39^{\circ}, 6 \mathrm{~N} ; \lambda=13^{\circ}, 1 \mathrm{E} ; \mathrm{h}=450 \mathrm{Km}: \mathrm{H}=20^{\mathrm{h}} 31^{\mathrm{m}} 33^{\mathrm{s}}$. 
Quest'ultimo sisma è notevole non solo per la sua grande profondità ipocentrale ma anche perchè il suo epicentro, a differenza di quelli relativi agli altri terremoti profondi tirrenici, i quali, com'è stato rilevato da Peterschmitt, si allineano quasi tutti lungo una fascia rettilinea passante per lo Stromboli e orientata da NNE a SSW, si trova quasi al centro del Tirreno, in una zona ove nessun focolare sismico s'era finora mai manifestato. Anche l'epicentro del terremoto del $1^{\circ}$ febbraio 1956 si trova un po' fuori, verso est, di tale allineamento.

52. 6 febbraio Zafferana Etnea (Catania)

Grado V

Alle $03^{\mathrm{h}} 55^{\mathrm{m}}$ eirca del 6 febbraio 1956 , una scossa sussultoria, valutata, per i suoi effetti, d'intensità pari al V grado Mercalli, fu avvertita a Zafferana Etnea, sul versante SE dell'Etna. Essa ebbe peró una propagazione limitatissima essendo stata percepita solamente nelle località limitrofe; a Macchia, distante da Zafferana appena sei chilometri, a Nicolosi, a Biancavilla il movimento tellurico non fu affatto avvertito.

\section{19 febbraio Monte $\mathrm{S}$. Angelo (Foggia) Grado $\mathrm{V}$}

Anche durante l'anno 1956 l'attività sismica nella regione garganica fu abbastanza vivace, per quanto assai meno intensa di quella manifestatasi durante l'anno precedente. Una prima scossa si ebbe il 24 gennaio alle ore $09^{\mathrm{h}}$ circa. Essa fu avvertita solo a Monte S. Angelo e nelle immediate vicinanze, ove risultó sussultoria e di leggera intensità (III grado). Un'altra scossa, pure leggera, fu avvertita nella stessa località alle $23^{\mathrm{l}} 30^{\mathrm{m}}$ cirea del 17 febbraio. Altre due, durante la giornata del 19 febbraio: la prima, leggera, alle $07^{\mathrm{h}} 45^{\mathrm{m}}$; la seconda, forte, alle $15^{\mathrm{h}} 49^{\mathrm{m}}$.

Quest'ultima scossa fu avvertita da tutta la popolazione di Monte S. Angelo, ma ebbe una propagazione molto limitata poichè nei paesi vicini fu percepita assai debolmente o passò del tutto inosservata.

\section{Argenta (Ferrara) - Battaglia Terme (Padova)}

20 febbraio

Grado V-VI

ПI 20 febbraio 1956 , qualche secondo prima delle $02^{\mathrm{l}} 30^{\mathrm{m}}$, una scossa di terremoto fu avvertita in varie località delle province di Padova, 


\section{$\lceil 54\rceil 20 \cdot$ Febbraio. 1956}

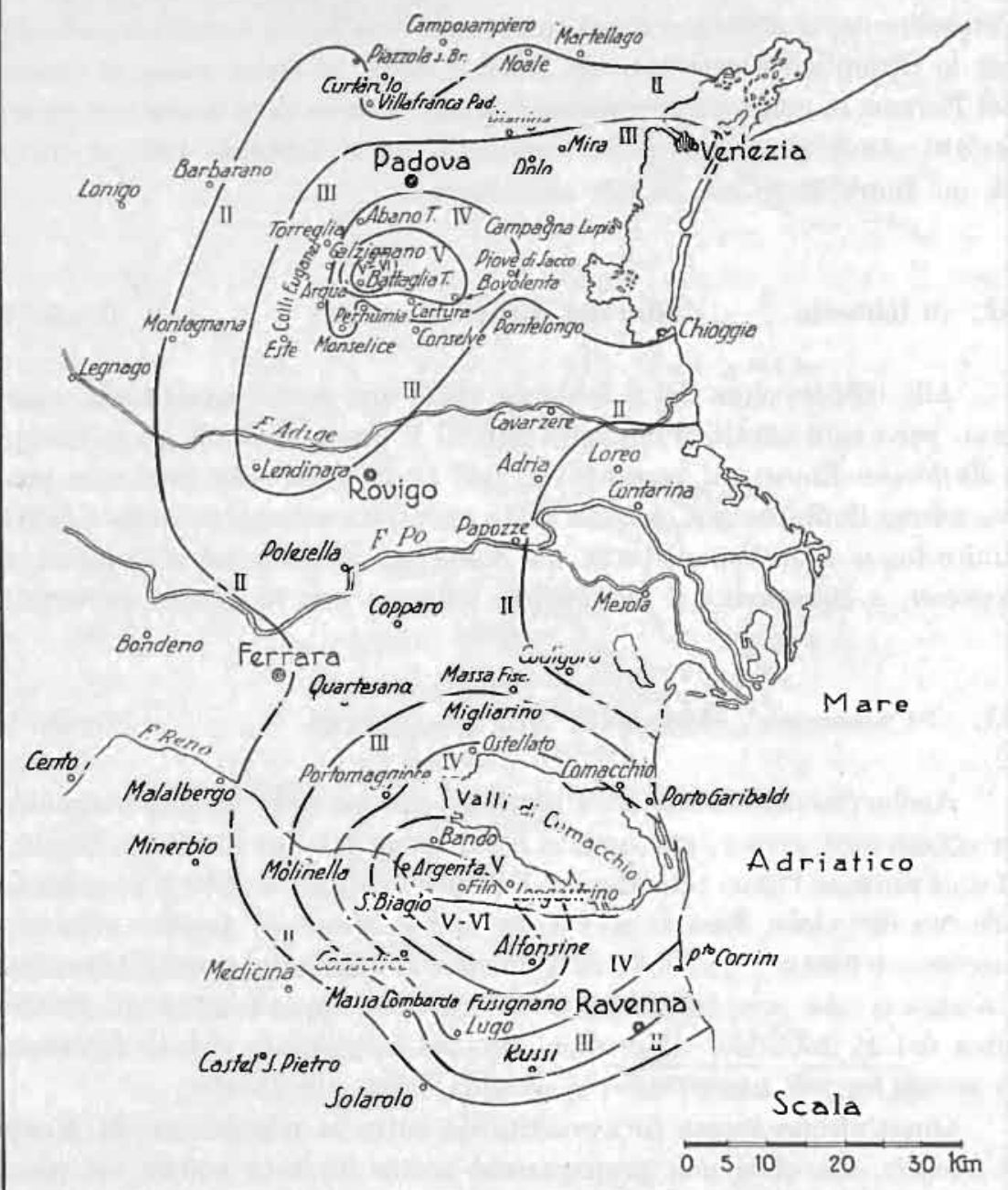

Fig. 32

Venezia, Rovigo, Ferrara e Ravenna. La figura 32 mostra la zona ove fu percepito il movimento tellurico.

Entro tale zona, assai singolare fu la distribuzione delle intensità: la maggiore (V-VI grado) si manifestò infatti in due aree separate $\mathrm{e}$ alquanto distanti tra di loro, come se il terremoto fosse stato gene- 
rato da due centri distinti entrati in attività quasi contemporaneamente. Se cosi fosse, il fenomeno non sarebbe nuovo per questa regione. Anche il terremoto dell'Alto Adriatico del 30 novembre 1930, come riuscì a stabilire P. Caloi in un approfondito studio su quell'interessante sisma $\left({ }^{7}\right)$, fu infatti originato da due diversi centri di scuotimento e $i$ due relativi epicentri risultarono nell'Alto Adriatico ad una cinquantina di chilometri l'uno dall'altro.

Le due aree predette, in cui il terremoto del 20 febbraio $1956 \mathrm{fu}$ avvertito con più forte intensità, sono situate, l'una nella parte meridionale e, l'altra, in quella settentrionale dell'intera zona perturbata macrosismicamente.

La prima, prossima alle Valli di Comacchio, comprende i comuni di Argenta (Ferrara) e di Alfonsine (Ravenna) ed appartiene ad una zona ben nota per la sua elevata sismicità. Nelle citate località furono avvertite due scosse sussultorie, separate da un intervallo di pochi secondi durante il quale fu udito un forte boato. Il movimento tellurico fu sensibile ad Ostellato, Porto Garibaldi, Lugo, Conselice; leggero a Comacchio, Portomaggiore, Molinella e Porto Corsini.

La seconda delle aree predette, situata ad oriente dei Colli Euganei, è assai meno estesa della prima e comprende il solo comune di Battaglia Terme (Padova). Anche qui furono avvertite da tutta la popolazione due scosse distinte, separate l'una dall'altra da un intervallo di qualche secondo. Intorno a tale area, il moto sismico fu avvertito fortemente a Bovolenta (Padova) e sensibilmente ad Abano Terme, Piove di Sacco, Monselice. La isosista d'intensità leggera, aperta sul Mare Adriatico, include le città di Venezia e di Padova con diversi comuni delle rispettive province, come Mira, Dolo, Chioggia, Campagna Lupia (della provincia di Venezia), Torreglia, Galzignano, Arquà Petrarca, Este, Conselve (della provincia di Padova) e si estende in provincia di Rovigo includendo il comune di Lendinara.

Il movimento tellurico fu registrato negli Osservatorî dell'Italia settentrionale e centrale e le ore relative all'inizio delle registrazioni sono le seguenti: Bologna $02^{\mathrm{h}} 29^{\mathrm{m}} 41^{\mathrm{s}}$; Padova $02^{\mathrm{h}} 49^{\mathrm{m}} 46^{\mathrm{s}}, 1$; Prato $02^{\mathrm{h}} 29^{\mathrm{m}} 48^{\mathrm{s}}$; Firenze $02^{\mathrm{h}} 29^{\mathrm{m}} 50^{\mathrm{s}}$; Saló $02^{\mathrm{h}} 30^{\mathrm{m}} 00^{\mathrm{s}}$; Pavia $02^{\mathrm{h}} 30^{\mathrm{m}} 07^{\mathrm{s}}$; Roma $02^{\mathrm{h}} 30^{\mathrm{m}} 30^{\mathrm{s}}$; Oropa $02^{\mathrm{h}} 30^{\mathrm{m}} 31^{\mathrm{s}}$.

Una replica di mediocre intensità, avvertita però solo nella zona compresa tra Alfonsine, Argenta e Conselice, si eb,be alle $04^{\mathrm{h}} 04^{\mathrm{m}}$ circa dello stesso giorno. 
Diversi centri sismici esistono nella regione del Lago di Garda e, più volte, da aleuni di essi irraggiarono nel passato violenti movimenti tellurici. Ad occidente del Lago, nei pressi di Salo, vi è il centro sismico da eui ebbe origine il rovinoso terremoto del 30 ottobre 1901 . Un altro, noto per un terremoto fortissimo e di vasta propagazione avvenuto il 14 aprile.1931, è situato in prossimità di Storo, fra la Valle del Chiese e

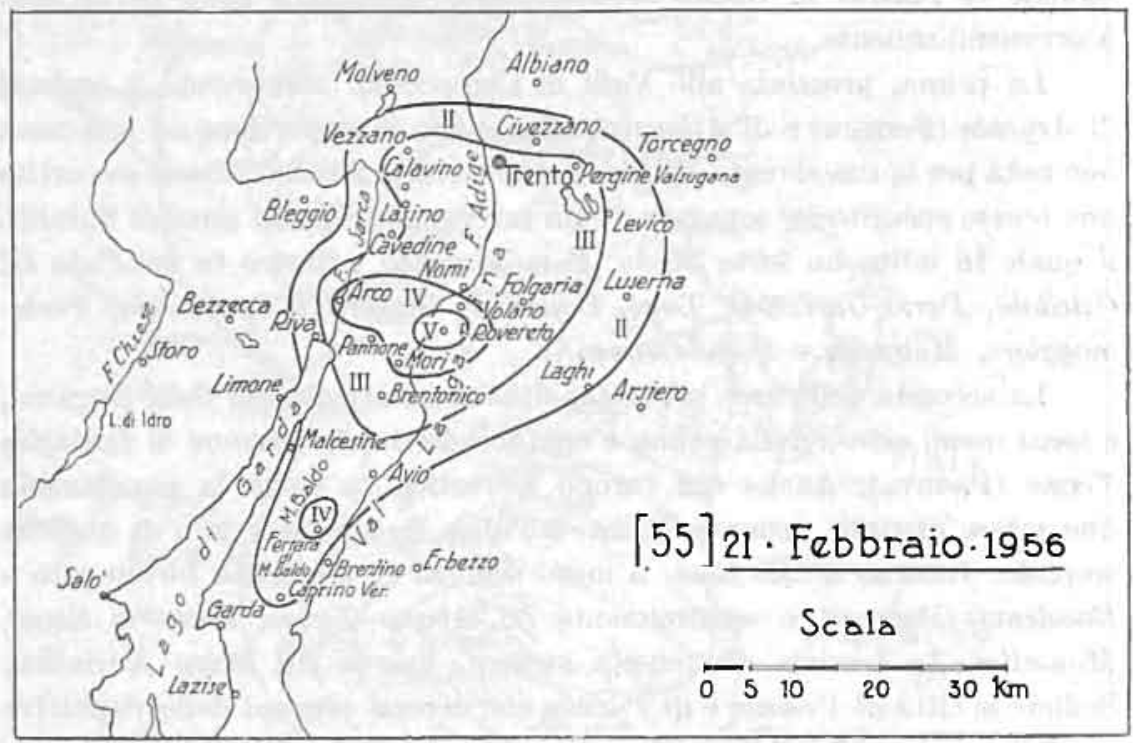

Fig. 33

quella del Sarca. Lungo la sponda veronese, la catena montuosa del Baldo, che divide il lago dalla Val Lagarina, costituisce una zona di elevata sismicità, con la serie numerosissima dei suoi terremoti per lo più di carattere locale, ma talora di elevata intensità, come ad esempio quello del 19 febbraio 1932, che riusci rovinoso a Lumini ed a S. Zeno. Un altro centro sismico si trova a sud del monte Baldo, in prossimità di Lazise: da esso ebbe origine una scossa fortissima il 21 giugno 1936. Altri centri di scuotimento si trovano presso la costa meridionale, da Desenzano a Peschiera.

Fra le zone adiacenti al Lago di Garda, la meno dotata di attività sismica è quella situata a nord del Monte Baldo, tra la Valle del Sarca 
e Ia Tal Lagarina. Per questa ragione, pur essendo stato di modesta intensità e di propagazione piuttosto limitata, acquista una certa importanza il sisma avvenuto appunto in tale zona alle $07^{\mathrm{h}} 15^{\mathrm{m}}$ del 21 febbraio 1956. Esso fu sentito come sussultorio e di $\mathrm{V}$ grado a Rovereto; di IV grado e sussultorio a Volano e a Mori; pure di IV grado ma ondulatorio ad Arco (fig. 33). Con intensità leggera (III grado) fu avvertito nella città di Trento e nei comuni di Vezzano, Pergine Valsugana, Calavino, Lasino, Folgaria e Brentonico.

Sulla riviera bresciana del Garda la scossa passò completamente inosservata, mentre su quella veronese fu percepita, sia pure molto lievemente, in quasi tutta la zona del Monte Baldo. È da rilevare anzi che, nella parte meridionale di questa zona, il movimento tellurico subì un sensibile aumento di intensità, essendo stato avvertito di IV grado intorno a Ferrara di Monte Baldo.

Non si ebbero repliche. Una lieve scossa premonitoria si era avuta alle $06^{\mathrm{h}} 30^{\mathrm{m}}$ circa dello stesso 21 febbraio.

Crediamo opportuno qui aggiungere che un movimento tellurico di mediocre intensità si era verificato nella stessa zona circa tre mesi prima della scossa di cui ora si è detto. Esso avvenne alle $07^{\mathrm{h}} 25^{\mathrm{m}}$ del 23 novembre 1955 e fu avvertito di IV grado a Rovereto e di III ad Areo.

56. 22 febbraio

Siena

Grado VI-VII

Un periodo sismico breve, ma di elevata frequenza, ebbe luogo nel territorio senese nei giorni 22 e 23 febbraio del 1956. Le scosse, anche le più intense, ebbero tutte una propagazione assai limitata e al loro epicentro, situato nelle immediate vicinanze di Siena, possono attribuirsi con buona approssimazione le coordinate $43^{\circ} 20^{\prime} \mathrm{N}$ e $11^{\circ} 20^{\prime} \mathrm{E}$.

Iniziò il periodo sismico una scossa di IV grado, della durata di 3 secondi, avvenuta alle $15^{\mathrm{h}} 08^{\mathrm{m}}$ circa del 22 febbraio. Seguirono numerose altre scosse di minore intensità, finchè alle $23^{\mathrm{h}} 55^{\mathrm{m}} 06^{\mathrm{s}}$ dello stesso giorno, accompagnata e seguita da forti rombi sotterranei, sopraggiunse la scossa prineipale del periodo sismico. Nella città di Siena, essa si manifestò con earattere nettamente sussultorio, ebbe una durata di 5 secondi e raggiunse un'intensità di VI-VII grado. Lo scuotimento, sentito fortemente da tutti nelle case, nei locali pubblici e sensibilmente anche nelle strade, provocò spavento generale. Moltissimi si riversarono nelle vie e nelle piazze, ivi pernottando nonostante la rigida temperatura invernale. Crollarono alcuni comignoli e Ieggere lesioni si verificarono in qualche edificio. 
Piccola dovette essere la profondità ipocentrale perchè, in relazione all'elevata intensità all'epicentro, la scossa ebbe, come dianzi si è accennato, una propagazione macrosismica assai modesta. Il movimento tellurico fu forte (V grado) a Sovicille, sensibile (IV grado) a Monteriggioni; poi, specialmente nel quadrante $\mathrm{SW}$, decrebbe rapidamente d'intensità, sicchè località non molto distanti da Siena some Murlo,

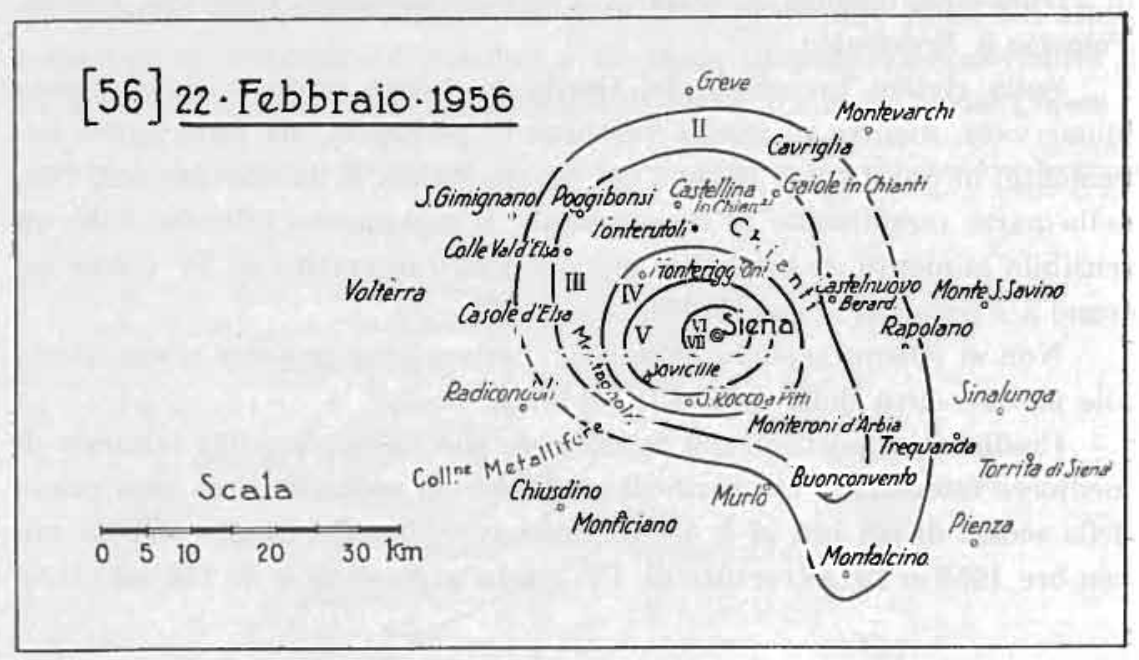

Fig. 34

Monticiano, Chiusdino, Radicondoli, Casole d'Elsa, non rilevarono affatto il fenomeno sismico. La fig. 34 mostra l'estensione della zona in cui la scossa fu percepita con leggera e con leggerissima intensità.

Registrazioni si ebbero, oltre che nell'Osservatorio di Poggio al Vento in Siena, anche negli Osservatori Ximeniano di Firenze e di S. Domenico in Prato.

Dopo la scossa di cui or ora si è detto, numerose ed a brevi intervalli di tempo l'una dall'altra continuarono le repliche durante la prima metà della giornata del 23 febbraio. Quasi tutte furono di leggera intensità. Due sensibili (IV grado) se ne ebbero rispettivamente alle $03^{\mathrm{h}} 51^{\mathrm{m}}$ e alle $07^{\mathrm{h}} 17^{\mathrm{m}}$ circa. Una abbastanza forte, sussultoria come le precedenti, avvertita da tutti con vivissimo spavento, si verifico alle $11^{\mathrm{h}} 08^{\mathrm{m}} 40^{\mathrm{s}}$. 
Fu valutata d'intensità fra il V ed il VI grado ed ebbe una durata di 3 secondi. La sua estensione macrosimica fu all'incirea uguale a quella della scossa avvenuta alle $23^{\mathrm{h}} 55^{\mathrm{m}}$ del giorno precedente. Verso ovest si propagò però maggiormente, essendo stata avvertita sensibilmente a Casole d'Elsa, dove le scosse del giorno avanti, compresa quella principale, erano passate del tutto inosservate.

Seguirono poche altre leggiere repliche; l'ultima si verificò alle $13^{\mathrm{h}} 5 \mathrm{~m}^{\mathrm{m}}$ circa del 23 febbraio.

58. 20 marzo

Civezza (Imperia)

Grado V

Il 20 marzo 1956 , alle $00^{\mathrm{h}} 35^{\mathrm{m}}$, una scossa di terremoto della durata di 5 secondi e valutata di $\mathrm{V}$ grado Mercalli, fu avvertita nel comune di Civezza, in provincia d'Imperia. In tutte le località circostanti il movi-

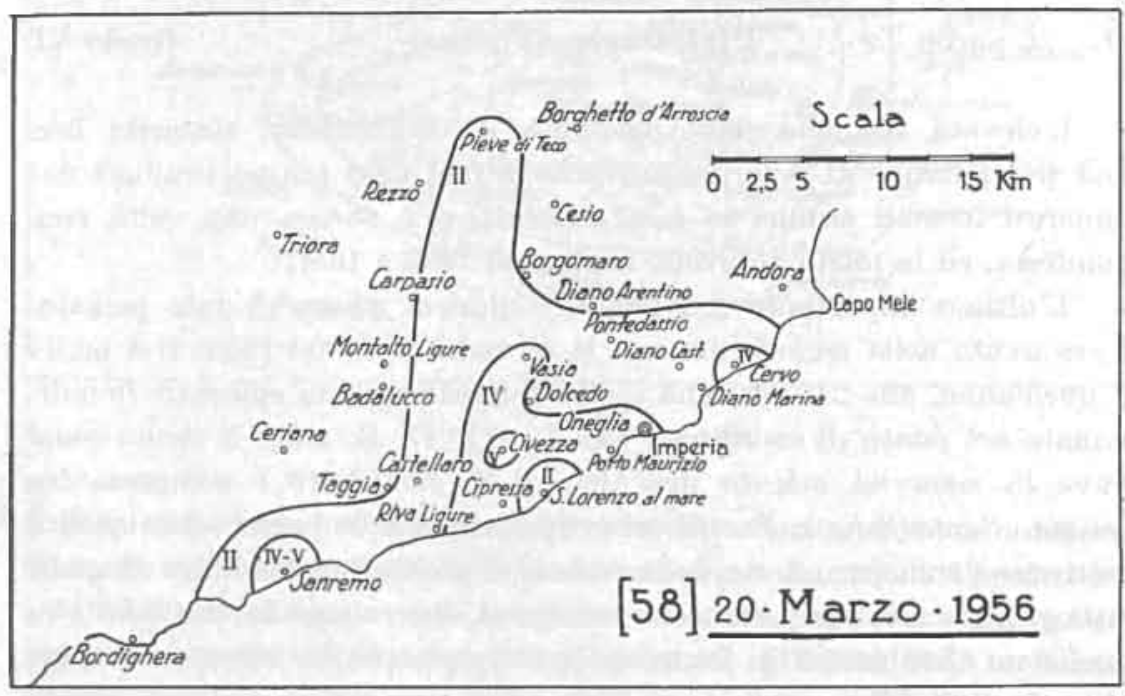

Fig. 35

mento tellurico fu avvertito più debolmente, ma la distribuzione assai anomala delle intensità rende incerta l'individuazione macrosismica dell'epicentro. Forse questo fu in mare e la maggiore intensità con cui il sisma si manifestò nel territorio di Civezza è da attribuire alla costituzione geologica del sottosuolo.

Nel paese di Cipressa, situato a sud di Civezza in prossimità della costa (fig. 35), l'intensità fu di IV grado e pure di IV ad Imperia; 
invece a San Lorenzo al Mare, fra Imperia e Cipressa, la scossa fu cosi lieve da passare inosservata alla quasi totalità degli abitanti.

Oltre che nelle predette località, la scossa venne nettamente avvertita nei comuni di Cervo e di Vasia. Nella eittà di Sanremo fu di brevissima durata ( 1 secondo) ma abbastanza forte ( $\mathrm{IV}-\mathrm{V}$ grado).

La zona ove il movimento tellurico fu percepito con lievissima intensità è limitata da una linea che, partendo dalla costa fra Sanremo e Bordighera, sale verso nord fino ad includere il comune di Pieve di Teco, poi, dopo aver descritto una profonda insenatura che lascia fuori Cesio, Borgomaro e Diano Arentino, torna al mare a sud di Capo Mele.

Nelle località ove risultò di V e di IV grado, la scossa venne giudicata come sussultoria e fu accompagnata da boati di lieve intensità.

Non si ebbero repliche.

59. 26 aprile

Pietramala (Firenze)

Grado VI

L'elevata sismicità dell'Appennino Tosco-Emiliano, sismicità ben nota per $i$ frequenti e fortissimi terremoti in ogni tempo irradiati dai numerosi focolari sismici in esso esistenti, si è ancora una volta resa manifesta, ed in modo notevole, negli anni 1956 e 1957.

L'ultimo importante movimento tellurico, prima di tale periodo, si era avuto nella regione durante la seconda metà del 1952. Il 4 luglio di quell'anno, alle $21^{\mathrm{h}} 35^{\mathrm{m}}$, una scossa fortissima, il cui epicentro fu individuato nel punto di coordinate $44^{\circ} \mathrm{N}$ e $11^{\circ} 47^{\prime} \mathrm{E}$, colpi in modo assai grave la zona ad oriente dell'Alpe di S. Benedetto e compresa fra Galeata, Santa Sofia e Premilcuore. Specialmente le prime due località soffrirono danni per effetto della scossa: si produssero fenditure in quasi tutti gli edifici, caddero numerosi comignoli, diverse case furono ridotte in condizioni d'inabitabilità. Piuttosto gravemente rimase lesionata l'antica Abbazia di S. Ellero, nelle immediate vicinanze di Galeata. Nei comuni di Predappio, Meldola, Portico di S. Benedetto, Dovadola, si ebbero lesioni gravi in edifici vecehi e leggiere anche in case di recente costruzione.

Questa scossa, di cui in fig. 36 è indicata la propagazione macrosismica nei limiti dell'intensità di IV grado, segnò l'mizio di un lungo periodo sismico durato, con una tregua di una quarantina di giorni, sino quasi alla fine dell'anno. Durante tutto il mese di luglio seguirono numerose repliche le quali, per quanto di intensità generalmente lieve, mantennero le popolazioni in continuo allarme, inducendole spesso ad abbandonare le abitazioni e ad accamparsi all'aperto. Tali scosse ebbero 
carattere locale e furono avvertite specialmente nella zona di Rocca S. Casciano. Molto frequenti nel mese di luglio, esse andarono diradando nei mesi successivi e il periodo sismico parve chiudersi con una leggera scossa avvertita nel comune di S. Pietro in Bagno il 13 ottobre 1952. II 2 dicembre, invece, nuove manifestazioni telluriche riportarono il punico fra le popolazioni dell'Appennino Tosco-Romagnolo. Alle $01^{\mathrm{h}} 30^{\mathrm{m}}$ di detto giorno fu avvertita una leggera scossa premonitoria e alle $07^{\mathrm{h}} 18^{\mathrm{m}}$

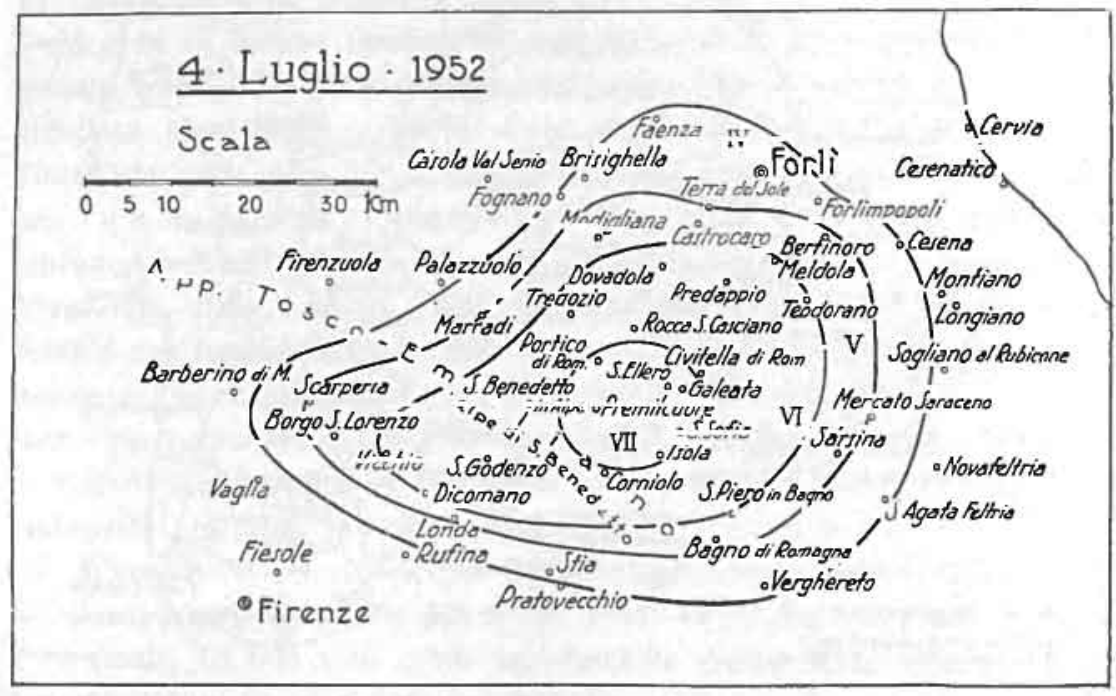

Fig. 36

si ebbe una scossa molto forte (VI grado) che raggiunse la sua massima intensità nel comune di Santa Sofia. In numerose abitazioni si produssero lievi fenditure ed alcune case coloniche, nella campagna ad est del paese, rimasero gravemente danneggiate. Nei pressi di Santa Sofia appunto, ad una distanza di una quindicina di chilometri da quello del terremoto del 4 luglio, si trovò l'epicentro. Altre repliche leggiere si susseguirono poi fino quasi alla fine di dicembre.

Dal principio del 1953 alla fine del 1955 , l'attività sismica nella regione dell'Appennino Tosco-Emiliano non fu rilevante: rare le scosse e tutte, eccetto quella del 29 marzo 1954 di cui si è detto al n. 20, di modesta intensità. Di esse ricorderemo qui la scossa, il cui epicentro fu probabilmente nei pressi di S. Pietro in Bagno, avvenuta alle $16^{\mathrm{h}} 15^{\mathrm{m}}$ circa del 21 dicembre 1954. A S. Pietro in Bagno essa risultò di IV-V grado e fu preceduta da un forte boato. Con intensità minore fu avvertita in 
varie località delle vallate del Savio, del Montone e del Bidente. La seguirono una decina di repliche durante la notte dal 21 al 22 dicembre.

Come si è prcedentemente accennato, assai rimarchevole è stata invece l'attività sismica nell'anzidetta regione durante gli anni 1956 e 1957.

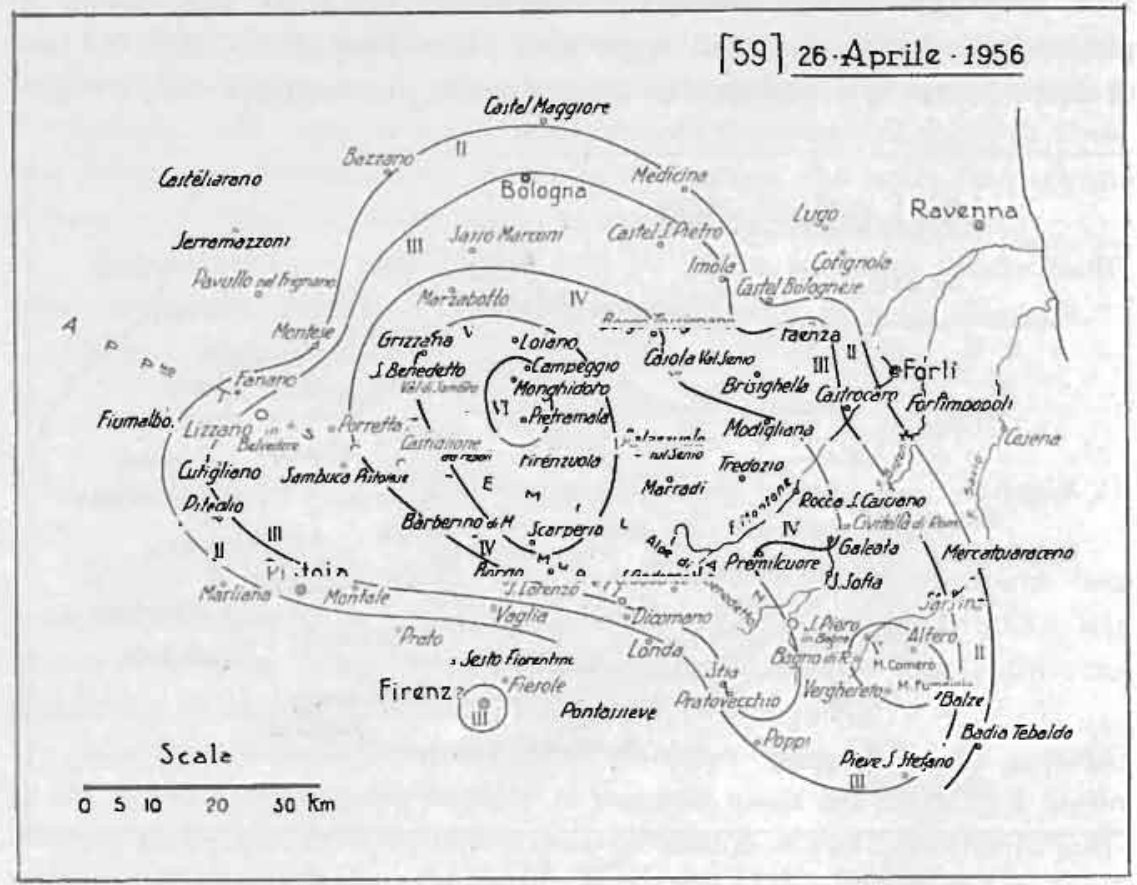

Fig. 37

Un periodo sismico, breve ma piuttosto intenso e caratterizzato anche da un'elevata frequenza, ebbe luogo nei giorni 25 e 26 aprile del 1956. Lo iniziò una leggera scossa premonitoria, avvertita a Pietramala (frazione del comune di Firenzuola) ed a Firenzuola, alle $17^{\mathrm{h}} 21^{\mathrm{m}}$ circa del 25 aprile. Altre scosse, di intensità non superiore al IV, si susseguirono numerose tino alle $03^{\text {h }}$ circa del giorno 26. Alcune di esse vennero avvertite, oltre che nelle predette località, anche a $S$. Benedetto Val di Sambro, a Castiglione dei Pepoli e nella zona del Mugello.

Alle $04^{\text {h }} 00^{\mathrm{m}} 03^{\text {s }}$ del giorno 26 , ebbe luogo la scossa principale che a Pietramala, Monghidoro e Campeggio raggiunse una intensità di VI grado e, con intensità di V, fu sentita nei comuni di S. Benedetto Val di Sambro, Grizzana, Loiano, Firenzuola e Scarperia. La figura 37 indica l'andamento delle isosiste tracciate in base alle notizie macrosismiche 
raccolte nelle varie località. L'area ove la scossa fu avvertita sensibilmente (IV grado) si estende nelle provincie di Bologna, di Forlì e di Firenze comprendendo i comuni di Marzabotto, Borgo Tossignano, Modigliana, Galeata, Palazzuolo sul Senio, Marradi, S. Godenzo, Pratovecchio, Borgo $S$. Lorenzo e Castiglione dei Pepoli. Il movimento fu percepito distintamente nelle città di Bologna e Firenze e, leggerissimo, anche nella città di Pistoia.

Nella zona di intensità molto forte, il movimento risultò sussultorio $\mathrm{e} f \mathrm{fu}$ in alcune località preceduto, in altre accompagnato, da un intenso rombo. La propagazione energetica della scossa fu, lungo alcune direzioni, assai discontinua. Si osservi nella figura 37 l'andamento dell'intensità nella direzione SE rispetto alla zona epicentrale: a Premilcuore e a Santa Sofia la scossa fu solo leggermente avvertita, mentre più lontano, in varie località attorno ai Monti Còmero e Fumaiolo, quali Verghereto, Balze, Alfero, Bagno di Romagna e S. Pietro in Bagno, fu sentita con una forte intensità. Verso sud il movimento si propagò fortemente fino a Scarperia; decrebbe poi rapidamente sì da passare del tutto inosservato a Sesto Fiorentino e a Fiesole; tornò ad aumentare di intensità nel territorio di Firenze ove fu distintamente percepito specialmente nei piani superiori degli edifici.

L'epicentro del sisma, per il quale nell'I.N.G., furono determinate le coordinate geografiche $44^{\circ} 09^{\prime} \mathrm{N}$ e $11^{\circ} 18^{\prime}, 7 \mathrm{E}$, cade nei pressi di Pietramala. In tale zona esiste in effetti un centro sismico già noto per manifestazioni da esso date nel passato.

Le repliche continuarono numerose fino alle ore 13 circa del 26 aprile. Furono tutte di mediocre e leggera intensità, eccetto una, avvenuta alle $07^{\mathrm{h}} 13^{\mathrm{m}} 30^{\mathrm{s}}$, che raggiunse quasi il $\mathrm{V}$ grado a Pietramala e fu avvertita in molte località fra cui Firenzuola, Barberino di Mugello e Castiglione dei Pepoli.

Quasi tutte le scosse furono registrate negli Osservatorî dell'Italia centrale. Quella principale anche negli Osservatorî dell'Italia settentrionale e meridionale.

60. 26 maggio Santa Sofia (Forli)

Grado VII

Era trascorso appena un mese dal breve periodo sismico di cui dianzi si è detto (59), quando un'altra serie di scosse, alcune fortissime, s'iniziava nell'Appennino Tosco-Emiliano colpendo però questa volta specialmente la zona ad oriente dell'Alpe di S. Benedetto situata fra le 
Alte Valli del Bidente e del Montone: la zona, cioè, già gravemente colpita dal terremoto del 4 luglio 1952.

Questo nuovo, intenso periodo sismico, originato da ipocentri alquanto distanti da quello da cui irradiarono le scosse del mese precedente, ebbe inizio il 25 maggio alle $20^{\mathrm{h}} 29^{\mathrm{m}}$ circa con una scossa leggerissima, registrata dai sismografi dell'Osservatorio di Prato ed avvertita da poche persone. Il giorno successivo, alle $18^{\mathrm{h}} 17^{\mathrm{m}}$, si ebbe un'altra scossa premo-

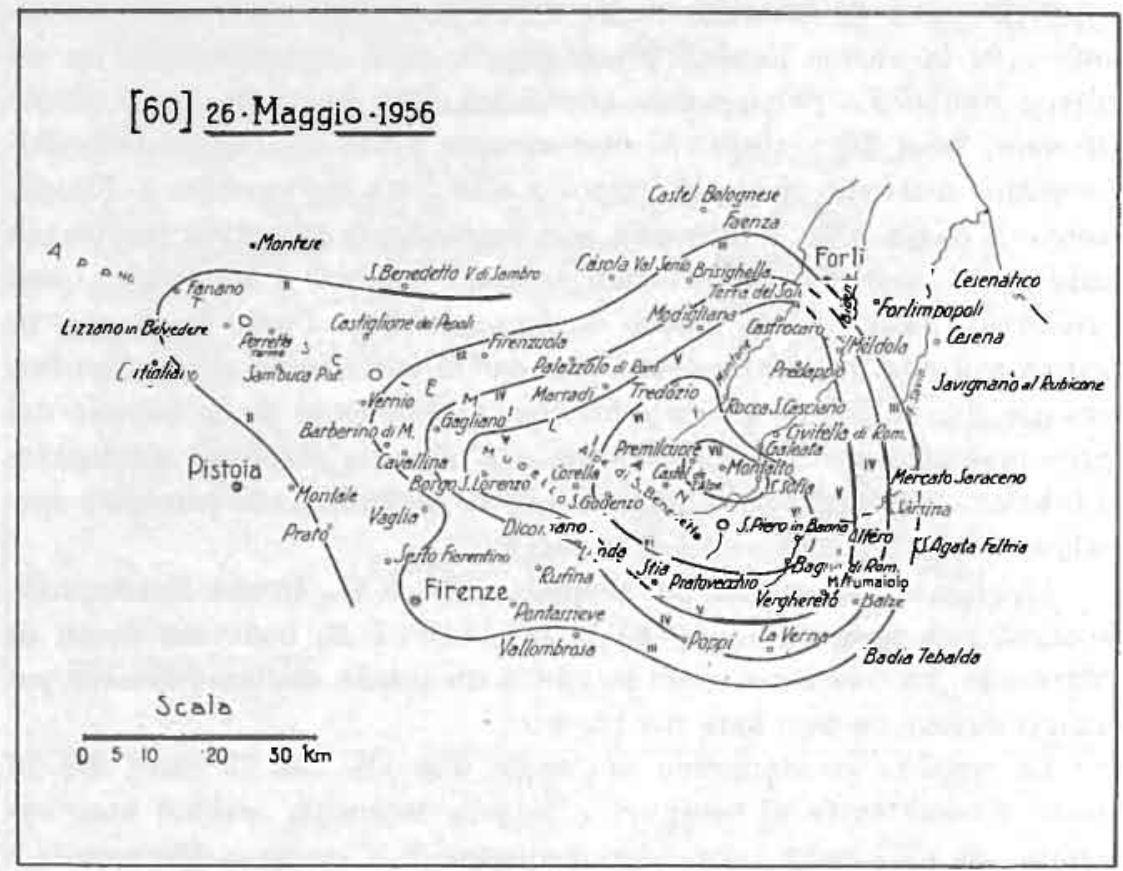

Fig. 38

nitoria di mediocre intensità ed infine, alle $19^{\mathrm{h}} 40^{\mathrm{m}}$, si verificò il primo massimo sismico della serie. La violenta scossa, accompagnata da un forte boato, urtò soprattutto la cittadina di Santa Sofia, raggiungendo ivi una intensità non inferiore al VII grado Mercalli: $\mathrm{i}$ vetri delle finestre andarono in frantumi, caddero lampadari dai soffitti, crollarono camini, danni gravi si verificarono in molti fabbricati. Gli abitanti furono presi da panico e immediatamente si riversarono all'aperto; memori delle distruzioni e delle vittime causate dal rovinoso terremoto del 10 novembre 1918, che nei pressi ebbe il suo epicentro, e dei gravi danni subiti per effetto del fortissimo terremoto sopra ricordato del 1952, si sistemarono sotto attendamenti apprestati nei piazzali, nei campi e dovunque 
vi fosse spazio sufficiente per evitare di essere travolti da un eventuale erollo di murature.

Anche a Premilcuore, distante da Santa Sofia una dozzina di chilometri, la scossa fu fortissima e causò molte lesioni negli edifici meno solidi. Tra le dette due località, all'incirca nel punto di coordinate $43^{\circ} 57^{\prime} \mathrm{N}$ e $11^{\circ} 47^{\prime} \mathrm{E}$, è probabilmente situato l'epicentro del sisma.

Il movimento tellurico fu molto forte a Tredozio, S. Godenzo, Castagno, San Piero in Bagno e Bagno di Romagna; forte in una zona avente ai limiti Stia, Pratovecchio, Verghereto, Castrocaro e Terra del Sole. La figura 38 mostra la propagazione macrosismica della scossa: propagazione piuttosto limitata in relazione alla fortissima intensità nella zona epicentrale e assai diversa nelle varie direzioni. Verso NE il movimento decrebbe dapprima rapidissimamente, tanto che a Galeata ed a Civitella di Romagna, località assai prossime all'epicentro, la scossa fu sentita solo di V grado; si mantenne poi forte fin nel territorio di Castrocaro e Terra del Sole, per diminuire di nuovo rapidamente estinguendosi del tutto nei pressi di Forli. La maggiore propagazione si ebbe nella direzione WNW, lungo la quale il movimento si estese fino a Fanano, in provincia di Modena.

Il terremoto fu registrato negli Osservatorî di Firenze, Prato, Pavia, Roma e in diversi altri dell'Italia centrale e settentrionale.

Dopo la violenta scossa del 26 maggio, vi fu una pausa di due giorni. I movimenti tellurici ricominciarono, nella stessa zona, alle prime ore del 29 maggio, culminando il 3 giugno in un'altra fortissima scossa di cui si dirà nelle pagine seguenti.

Il $1^{\circ}$ giugno 1956 , alle $03^{\mathrm{h}} 30^{\mathrm{m}}$ e alle $09^{\mathrm{h}} 33^{\mathrm{m}}$ circa rispettivamente, ebbero luogo nelle Alpi Piemontesi due scosse di terremoto.

La prima fu avvertita con mediocre intensità nel comune di Oncino (Cuneo) e, più leggermente, in varie località delle provincie di Cuneo e di Torino, tra le quali Envie, Ostana, Castelmagno, Pinerolo, S. Secondo di Pinerolo e Torre Pellice.

La seconda, molto forte, esplicò la sua maggiore intensità (VI grado) nei territori comunali di Envie e di Oncino nell'Alta Valle del Po. In tali località la scossa, preceduta da rombo ed avvertita da tutti, provocò caduta di calcinacci e leggere lesioni in alcuni edifici. Questo movimento tellurico, che interessò una larga parte delle province di Cuneo e di Torino, fu earatterizzato da notevoli anomalie nella distribuzione delle 
intensità. Così, ad esempio, fra Envie ed Oncino, cioè fra le due località ove la scossa raggiunse il VI grado, vi fu una zona ove l'intensità risultò alquanto minore; come se due centri distinti di scuotimento fossero entrati in azione nello stesso tempo o quasi. Vi furono paesi, come Crissolo e Barge, distanti pochi chilometri dai centri più colpiti, ove la scossa

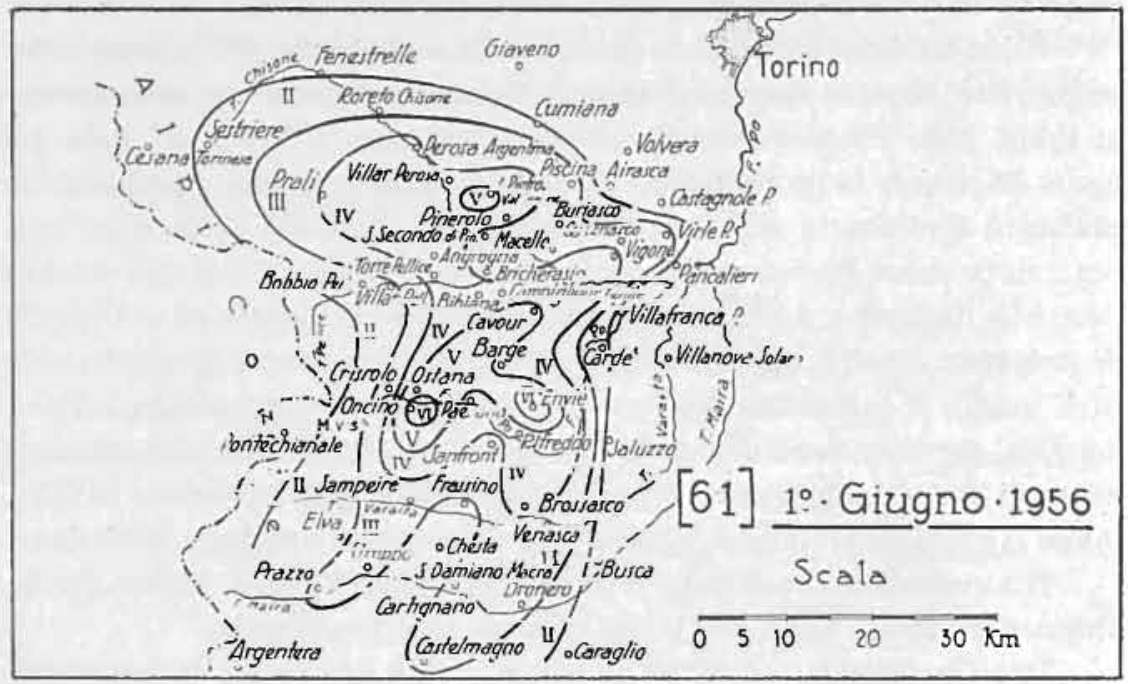

Fig. 39

venne giudicata leggera, mentre in altri molto più distanti, come Cavour, in provincia di Torino, la scossa venne valutata di forte intensità. Altra singolarità presentò la zona di San Pietro Val Lèmina (Torino), ove il fenomeno sismico fu giudicato di $\mathrm{V}$ grado mentre in tutti i paesi circostanti risultò di IV ed anche di III grado. Ed aneora: verso sud il movimento si propagò sensibilmente fino a Prazzo e Castelmagno (Cuneo), ma fra questi paesi e quelli di Oncino ed Envie ne troviamo altri, per esempio Frassino, ove la scossa passò quasi inosservata.

Queste anomalie appaiono dalla figura 39 , nella quale abbiamo tentato di tracciare le irregolarissime isosiste sulla scorta delle numerose notizie raccolte dalle varie località.

Fra gli Osservatorî italiani, registrarono il sisma quelli di Oropa, di Pavia, di Bologna e di Firenze. Ora origine: $09^{\mathrm{h}} 32^{\mathrm{m}} 45^{\mathrm{s}}$.

Alle $11^{\text {h }} 15^{\mathrm{m}}$ dello stesso giorno fu avvertita a Saluzzo una scossa di carattere sussultorio, valutata di IV-V grado Mercalli. Dovette trattarsi però di un fenomeno locale, poichè non risulta che la scossa sia stata avvertita nei paesi vicini. 
Dopo la scossa del 26 maggio 1956, cui seguirono due giorni di pausa, i movimenti tellurici dell'Appennino Tosco-Emiliano ripresero nelle prime ore del 29 maggio.

Una scossa di mediocre intensità si verificò alle $00^{\mathrm{h}} 56^{\mathrm{m}}$; un'altra abbastanza forte, registrata negli Osservatorî dell'Italia centrale ed avvertita sensibilmente in numerosi centri dell'Appennino Tosco-Romagnolo, fra i quali Santa Sofia, S. Piero in Bagno, Civitella di Romagna, Galeata e Meldola, si ebbe alle $17^{\mathrm{h}} 21^{\mathrm{m}}$. Altre scosse, leggerissime e leggiere, seguirono durante le giornate del 30,31 maggio e del $1^{\circ}$ giugno. Una forte se ne verificò alle $22^{\mathrm{h}} 36^{\mathrm{m}}$ del 2 giugno, avvertita di $\mathrm{V}$ grado a Santa Sofia e, con intensità via via descrescente con la distanza, in una zona avente ai limiti Marradi, Rocca San Casciano, Verghereto, Pratovecchio e S. Godenzo.

Alle $02^{\mathrm{h}} 46^{\mathrm{m}}$ circa del 3 giugno sopravvenne il secondo massimo del periodo sismico iniziatosi, come s'è visto, alle $20^{\mathrm{h}} 29^{\mathrm{m}}$ del 25 maggio. La scossa fu fortissima (VII grado) e colpi, questa volta, specialmente Rocca San Casciano. Nei pressi di questa cittadina, situata sulle rive del Fiume Montone, una quindicina di chilometri a nord di Santa Sofia, crediamo possa essere, con buona probabilità, ubicato l'epicentro, assegnando alle sue coordinate geografiche i valori approssimati di $44^{\circ} 04^{\prime} \mathrm{N}$ e $11^{\circ} 49^{\prime} \mathrm{E}$. In tale cittadina, infatti, la scossa, sussultoria e accompagnata da forti rombi, sviluppò la sua maggiore intensità, generando vivissimo panico nella popolazione e causando lesioni gravi in molte abitazioni.

A Santa Sofia il movimento tellurico fu forte, ondulatorio, e aggravò le lesioni causate dalle precedenti scosse. Pure fortemente fu sentito a Marradi, Galeata, S. Piero in Bagno e Verghereto. Anche in questo caso, le onde sismiche si propagarono verso ovest maggiormente che nelle altre direzioni, giungendo con lieve intensità fin nella provincia di Modena (v. fig. 40).

Il terremoto fu registrato in molti Osservatori, fra i quali quelli di Firenze, Prato, Bologna, Roma e Pavia.

Varie repliche, d'intensità non superiore al IV grado, si susseguirono fino al 5 giugno.

Il 21 di giugno, alle $00^{\mathrm{h}} 23^{\mathrm{m}}$, ricominciarono le scosse nella zona di Santa Sofia e continuarono, mantenendosi però sempre di leggera intensità, fino al 29 giugno. Due altre repliche, sempre a Santa Sofia, si ebbero nella giornata del 27 luglio: la prima alle $11^{\mathrm{h}} 10^{\mathrm{m}}$, di III grado; la seconda, alle $11^{\mathrm{th}} 20^{\mathrm{m}}$, di IV. 
A completare le notizie sopra riferite, ricorderemo le seguenti altre scosse avvenute nel 1956 nelle zone di Firenzuola e di Santa Sofia e la cui intensità non superó il IV grado Mercalli. Nella zona di Firenzuola si ebbero tre scosse di III-IV grado il 5 settembre alle $00^{\mathrm{h}} 36^{\mathrm{m}}, 01^{\mathrm{h}} 35^{\mathrm{m}} \mathrm{e}$

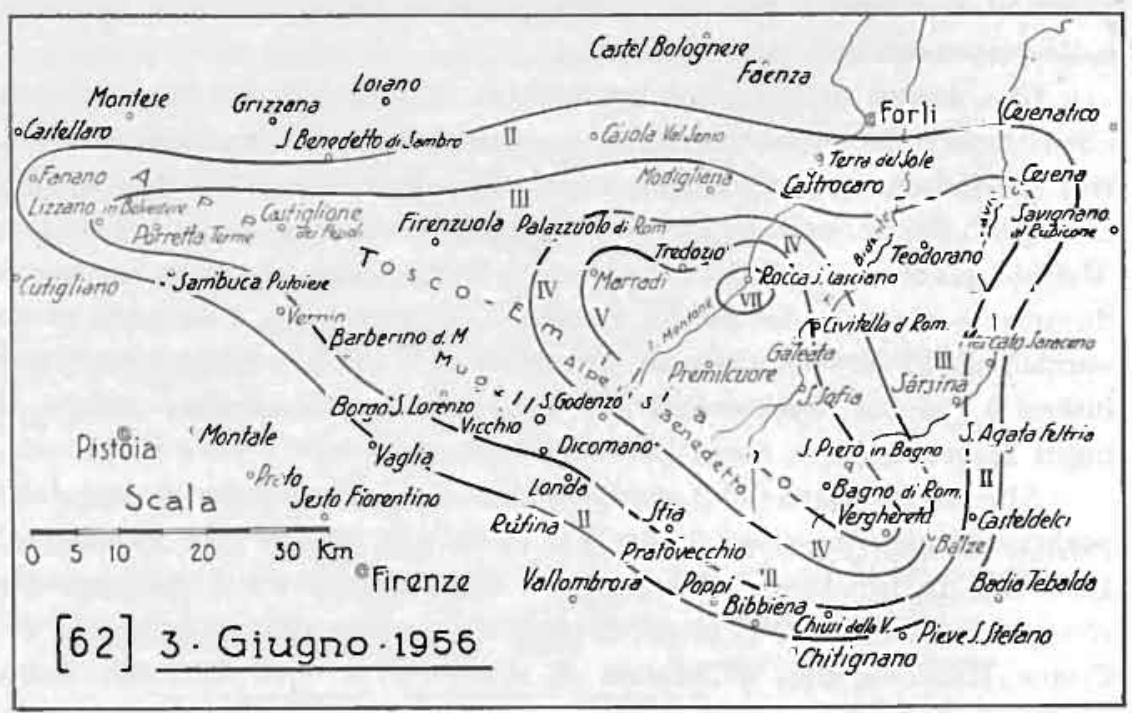

Fig. 40

$06^{\mathrm{h}} 11^{\mathrm{m}}$ rispettivamente. Nella zona di Santa Sofia si verificarono: una scossa di IV grado alle $02^{\mathrm{h}} 40^{\mathrm{m}}$ del 17 novembre; una di III, alle $14^{\mathrm{h}} 00^{\mathrm{m}}$ del 26 novembre; una di IV, alle $23^{\mathrm{h}} 50^{\mathrm{m}}$ del 29 novembre ed una ancora di III alle $17^{\mathrm{h}} 00^{\mathrm{m}}$ del 30 novembre. Con esse si chiude la lunga serie dei moti sismici avvenuti nell'Appennino Tosco-romagnolo durante il $1956 \mathrm{e}$ irradiati, come s'è visto, da tre distinti centri di scuotimento situati rispettivamente nei pressi di Pietramala, Santa Sofia e Rocca San Casciano.

63. 23 luglio

Monte S. Angelo (Foggia)

Grado V-VI

Notevole fu, anche nell'anno 1956, l'attività sismica nella regione garganica. Alcune scosse non molto intense ebbero luogo, come si è visto nelle pagine precedenti (53), durante i mesi di gennaio e di febbraio. Numerose altre se ne verificarono dal luglio al settembre e alcune di esse furono d'intensità piuttosto elevata, anche se, in generale, i loro effetti rimasero localizzati a territori di limitata estensione. 
Una prima scossa fu avvertita a Monte Sant'Angelo alle $01^{\mathrm{h}} 30^{\mathrm{m}}$ del 19 luglio e fu abbastanza forte da provocare il risveglio di molte persone addormentate.

Il 23 luglio, alle $13^{\mathrm{h}} 54^{\mathrm{m}}$, ebbe luogo una scossa la cui intensità, a Monte Sant'Angelo, raggiunse il V-VI grado. Ivi fu avvertita da moltissimi nelle strade e da tutti nelle case, con spavento e fuga di alcuni all'aperto.

Nella predetta eittadina il moto sismico ebbe carattere prevalentemente sussultorio; nelle località circostanti invece fu avvertito come ondulatorio e con intensità alquanto inferiore. È da ritenere pertanto che nei pressi di Monte S. Angelo debba essere stato l'epicentro.

Una replica di IV-V grado si ebbe alle $01^{\mathrm{h}} 50^{\mathrm{m}}$ del giorno successivo e una di III alle $20^{\mathrm{h}} 06^{\mathrm{m}}$ del 28 luglio. Nessun Osservatorio registrò i movimenti sismici predetti, i quali ebbero tutti un carattere strettamente locale.

64. 16 agosto

Una scossa sismica sussultoria, giudicata d'intensità pari al V grado Mercalli, fu avvertita, alle $09^{\mathrm{h}} 35^{\mathrm{m}}$ eirca del 16 agosto, nel comune di Cittareale, in provincia di Rieti. Il movimento tellurico ebbe carattere strettamente locale: fu avvertito infatti mediocremente solo in una ristretta zona intorno a Cittareale, comprendente le frazioni di S. Giusta, Santa Croce e Trimezzo, poi decrebbe rapidamente d'intensità cosi da ridursi al II grado a Leonessa, a Borbona, ad Amatrice ed in altre località situate tutte a breve distanza da Cittareale.

Una replica di minore intensità (IV-V grado) si verificò circa tre ore dopo, alle $12^{\mathrm{h}} 23^{\mathrm{m}}$. Le due scosse furono registrate negli Osservatorî di Roma e di Firenze.

\section{17 agosto San Giovanni Rotondo (Foggia) Grado VI-VII}

Dopo le seosse avvenute dal 19 al 28 luglio 1956, con epicentro nei pressi di Monte Sant'Angelo (63), l'attività sismica nella regione del Gargano s'acquietò per una quindicina di giorni. Riprese alle $12^{\mathrm{h}} 55^{\mathrm{m}}$ del 15 agosto con una leggera scossa avvertita a Monte Sant'Angelo e in altre località del Promontorio garganico. 
Il 17 agosto, la cittadina di S. Giovanni Rotondo, situata una ventina di chilometri ad ovest di Monte Sant'Angelo, fu colpita da una violenta scossa sussultoria che, provocando uno spavento generale nella popolazione, fece crollare alcuni muri di cinta nella periferia dell'abitato e causò lesioni sensibili in numerosi edifici. La scossa fu accompagnata da rombi intensi e prolungati. Il suo epicentro fu con ogni probabilità nei pressi di S. Giovanni Rotondo poichè ivi essa manifestò la sua maggiore intensità (VI-VII grado).

Non abbiamo potuto avere su questo movimento tellurico un numero di notizie sufficiente per poterne tracciare le isosiste; da quelle pervenuteci, tuttavia, sembra potersi dedurre che la sua propagazione fu alquanto limitata. $\mathrm{Fu}$ avvertito infatti fortemente (V grado) a San Marco in Lamis e sensibilmente (IV grado) a Rignano Garganico, località le cui distanze da San Giovanni Rotondo non superano, in linea d'aria, rispettivamente gli otto e $\mathrm{i}$ dodici chilometri. In altri centri del Gargano o non fu percepito affatto o lo fu solo con intensità leggera $\mathrm{e}$ leggerissima.

Un'altra scossa, d'intensità però assai modesta, fu avvertita alle $12^{\text {h }} 55^{\mathrm{m}}$ del 19 agosto sulla costa orientale del Gargano. A Vieste, in eui, come sembra, risultó più intensa che altrove, raggiunse solo il IV grado Mercalli. Probabilmente si tratta di una lieve manifestazione di un centro sismico sottomarino situato in prossimità della costa orientale del Gargano.

Nell'ultima decade di agosto non si ebbero nel Gargano altri movimenti tellurici di apprezzabile entità. Ricominciarono il $1^{\circ}$ settembre per culminare il giorno 22 in un terremoto che può considerarsi come il più importante fra quelli avvenuti in detta regione nell'anno 1956 (v.n. 67).

\section{6. $1^{0}$ settembre Monte S. Angelo (Foggia) Grado V-VI}

Questa scossa, verificatasi alle $23^{\mathrm{h}} 57^{\mathrm{m}}$, ebbe, come le precedenti, una propagazione assai limitata. A Monte Sant'Angelo, ove la sua intensità fu valutata di V-VI grado, fu avvertita da tutti nelle case, con risveglio di persone addormentate, e da molti con vivo spavento. Non si ebbero danni: solo caduta d'intonaci da murature forse già lesionate dai precedenti movimenti tellurici. Pensiamo che l'epicentro possa essere posto in prossimità di Monte Sant'Angelo poichè, per quanto ci risulta, nelle località vicine il fenomeno sismico fu avvertito con intensità nettamente inferiore. 
Una replica leggera ebbe luogo alle $18^{\mathrm{h}} 50^{\mathrm{m}}$ del 4 settembre ed un'altra, mediocre, il 18 settembre alle $21^{\mathrm{h}} 10^{\mathrm{m}}$. Il 16 settembre poi, alle $10^{\mathrm{h}} 43^{\mathrm{m}}$ circa, un movimento tellurico fu avvertito nel Tavoliere di Puglia e nell'estremità occidentale del Massiccio garganico. In base alle notizie pervenuteci sembra che la sua massima intensità (Foggia, Rignano Garganico) non abbia superato il IV grado. Sotto forma microsismica esso si propagò però notevolmente e se ne ebbero registrazioni negli Osservatorî di Messina, Taranto e Firenze.

67. 22 settembre

È questoil più notevole, non tanto per l'intensità quanto per la vasta propagazione macro e microsismica, fra $\mathrm{i}$ terremoti avvenuti nelle Puglie durante l'anno 1956. Esso colpi specialmente la provincia di Foggia ma fu fortemente sentito in provincia di Bari e, con leggera intensità, venne avvertito anche in alcune zone delle province di Avellino e di Campobasso. Se ne ebbero registrazioni in tutti gli Osservatorî italiani, da quelli della Sicilia a quelli del Piemonte, della Lombardia e del Veneto. L'ora origine dedotta da tali registrazioni fu $04^{\mathrm{h}} 19^{\mathrm{m}} 39^{\mathrm{s}}$.

La zona più colpita fu ancora quella di S. Giovanni Rotondo. Ivi il movimento sismico, sussultorio e accompagnato da rombi, raggiunse l'intensità di VI grado, causando fenditure leggiere in molte case. A Monte Sant'Angelo e a San Marco in Lamis fu avvertito come sussultorioondulatorio e valutato di un'intensità di V-VI grado. Probabilmente l'epicentro fu fra San Giovanni Rotondo e Monte Sant'Angelo, più prossimo però alla prima che alla seconda località.

La zona ove la scossa fu avvertita con forte intensità è assai vasta, estendendosi su gran parte del Promontorio garganico e su tutta la Capitanata. L'isosista che la circoserive comprende infatti i comuni di Vieste, sull'estrema punta orientale del Gargano, di Peschici, Rignano Garganico, San Severo, Lucera, Cerignola (tutti della provincia di Foggia), e Canosa di Puglia, in provincia di Bari. Anche a Foggia il terremoto fu fortemente sentito e, come negli altri centri menzionati, causò vivo spavento. Moltissimi, svegliati dall'improvvisa scossa si riversarono nelle strade, nelle piazze e nei campi, rimanendovi per tutto il resto della mattinata.

La propagazione dell'energia sismica, come generalmente accade per i terremoti originati dagli ipocentri della regione garganica, avvenne prevalentemente in direzione SSE, Iungo la Capitanata (fig. 41). Modesta 
fu invece la propagazione verso la zona litoranea compresa fra Campomarino (Campobasso) e Rodi Garganico (Foggia). In questa zona, infatti, Il movimento sismico fu avvertito solo con leggerissima intensità. È però da rilevare un'anomalia verificatasi in una ristretta area intorno al paese di Poggio Imperiale: mentre nelle località circostanti il moto sismico fu

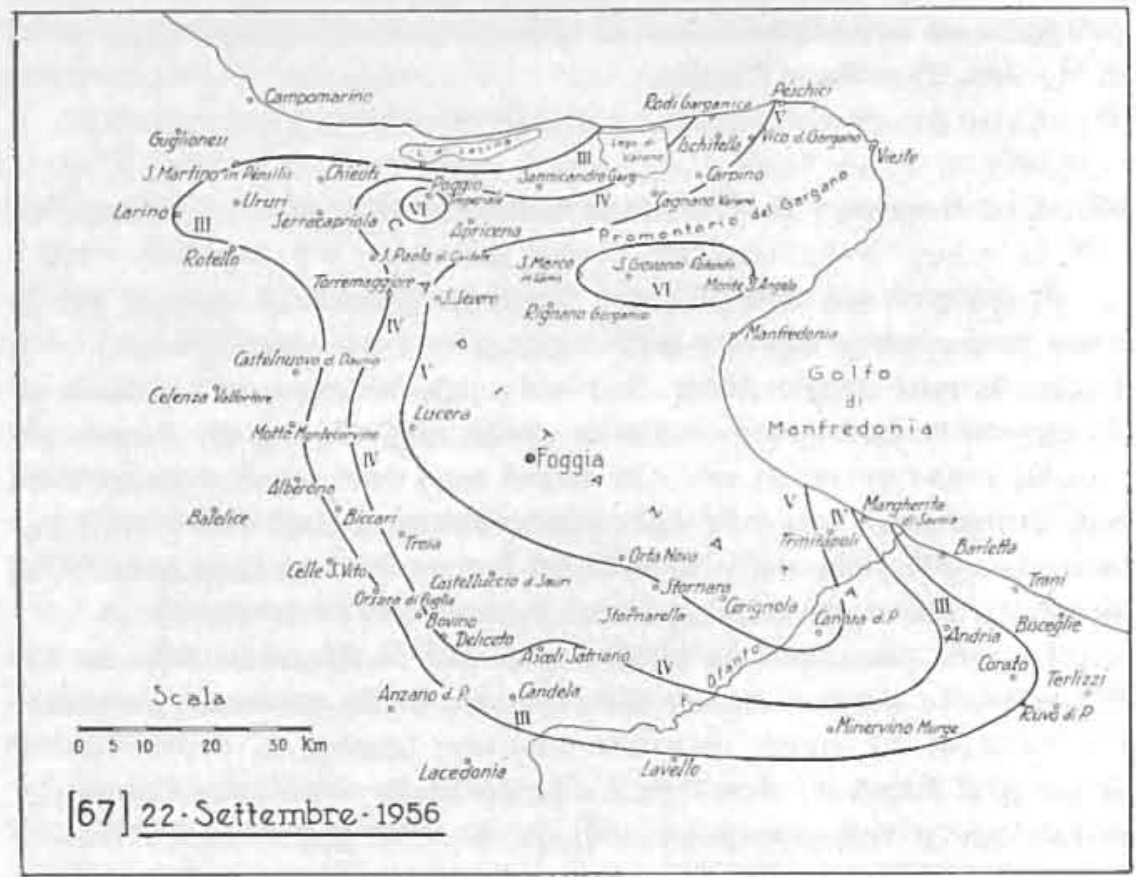

Fig. 41

mediocremente avvertito, a Poggio Imperiale l'intensità fu giudicata molto forte e valutata di VI grado. A Lesina, situata sulla sponda meridionale del Lago omonimo, pochi chilometri a nord di Poggio Imperiale, la scossa passó quasi inosservata.

Nella figura 41 sono indicate le isosiste del sisma fino a quella di III grado. E pressochè impossibile formarsi un'idea anche solo approssimata della isosista corrispondente al II grado e quindi della totalità della zona macrosismicamente interessata dal movimento tellurico. Si ebbero infatti notizie da luoghi molto distanti dalla zona epicentrale, come per esempio dalle città di Napoli e di Bari, secondo le quali il movimento sismico sarebbe stato colà percepito, sia pure in modo leggerissimo. In moltissimi altri invece, fra i quali citiamo Barletta, Terlizzi, Ruvo di 
Puglia, Anzano di Puglia, Celle S. Vito, Castelnuovo della Daunia, Rotello, Palata, tutti esterni alla zona isosismica leggera ma assai prossimi ad essa, Ia scossa non venne affatto avvertita.

Poche e lievi repliche si susseguirono subito dopo la scossa principale. L'ultima un po' più forte delle altre, si verifico alle $06^{\mathrm{h}} 30^{\mathrm{m}}$ dello stesso giorno, 22 settembre.

68. 3 ottobre Caramanico - Tocco da Casauria (Pescara) Grado V

Questo terremoto, avvenuto alle $01^{\mathrm{h}} 34^{\mathrm{m}}$ circa del 3 ottobre 1956 , ebbe il suo epicentro sulle pendici orientali del Morrone, probabilmente

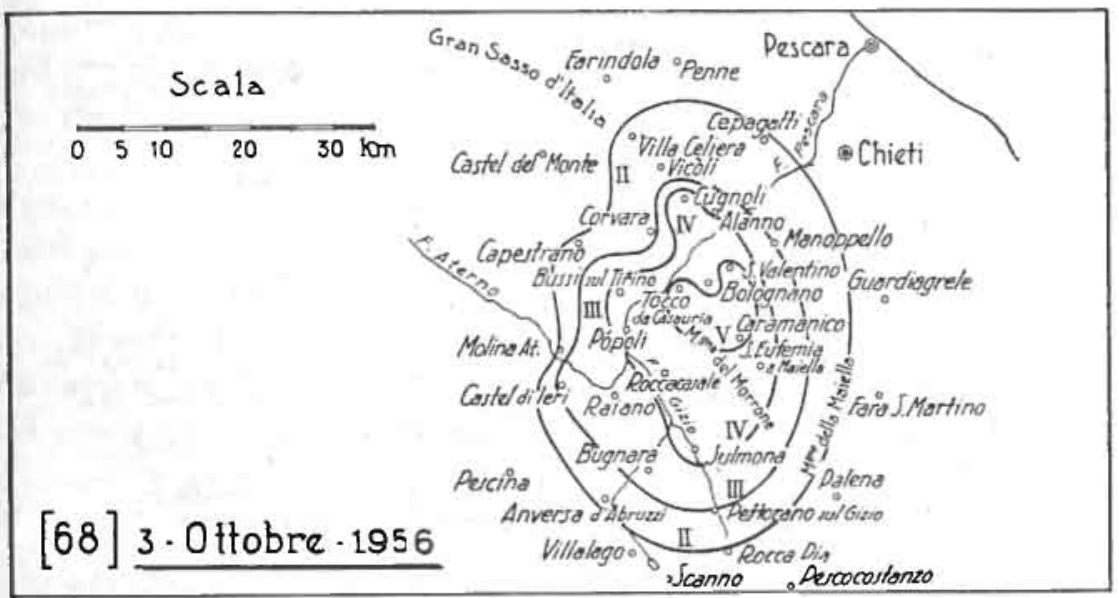

Fig. 42

nei pressi del punto di coordinate $42^{\circ} 12^{\prime} \mathrm{N}$ e $13^{\circ} 59^{\prime} \mathrm{E}$, fra Tocco da Casauria e Caramanico. In questi due paesi e a San Valentino la scossa si manifestò con un'intensità di V grado. Più leggera (IV grado) fu invece giudicata a Bolognano, Iocalità situata fra Tocco da Casauria e Caramanico; pure di IV grado fu avvertita nella città di Sulmona e nei comuni di Sant'Eufemia a Maiella, Roccacasale, Popoli, Bussi sul Tirino e Cugnoli.

La fig. 42 dà Ie isosiste del movimento tellurico tracciate in base alle informazioni macrosismiche ricevute.

La scossa fu registrata nell'Osservatorio sismico di Roma. Non fu segnalata alcuna replica. 
Il 5 novembre 1956 , alle $20^{\mathrm{h}} 45^{\mathrm{m}}$, un terremoto molto forte accompagnato da un cupo boato si abbattè all'improvviso sulle Alpi Carniche, nella zona a sud dei Monti Crostis e Paularo, in prossnnità del confine con l'Austria. Le località maggiormente colpite furono Paluzza, Treppo Carnico, Ligosullo, Comeglians ed Arta, tutte in provincia di Udine. In

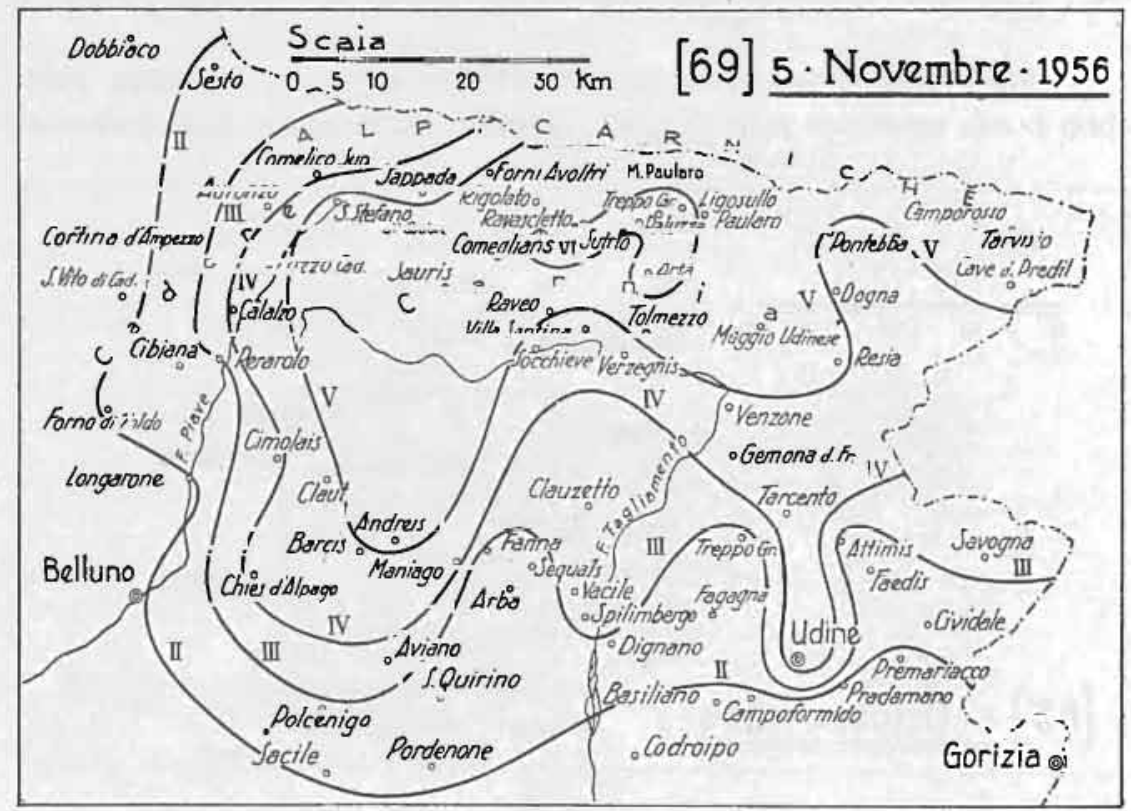

Fig. 43

esse la scossa raggiunse un'intensità di VI grado e causò cadute di calcinacci, crolli di comignoll e fenditure in numerose case. Con un'mtensità un po' minore (V-VI) grado si manifestò a Sutrio, nelle sue frazioni di Priola e Noiaris e nel comune di Ravascletto.

In tutte le località sopra menzionate la scossa fu avvertita con carattere nettamente sussultorio ed ebbe una durata di 5-6 secondi.

Mancando di notizie relative ai paesi di oltre confine, ci limitiamo a dare, nella figura 43 , le isosiste tracciate in base alle informazioni pervenuteci dal territorio italiano. In questo, il movimento tellurico ebbe una propagazione abbastanza vasta (avendo interessato più o meno 
sensibilmente quasi tutto il Friuli ed una parte della provincia di Belluno), ma, come appare dalla figura, grandemente irregolare. A Raveo e a Tolmezzo, per esempio, la scossa fu sentita fortemente mentre nel paese di Villa Santina, situato fra i primi due, lo fu solo mediocremente. La isosista di $\mathrm{V}$ grado, marcata da profonde insenature, si estende verso sud fino alla zona di Andreis ed ha ai suoi limiti i paesi di Forni Avoltri, S. Stefano di Cadore, Andreis, Raveo, Tolmezzo, Resia e Cave del Predil (frazione di Tarvisio). Nella eittà di Udine la scossa fu sensibile mentre passò inosservata nei vicini paesi di Campoformido e Pradamano.

Le coordinate epicentrali del sisma determinate dall'I.N.G. $\left(46^{\circ} 32^{\prime} \mathrm{N}\right.$ e $13^{\circ} 05^{\prime} \mathrm{E}$ ) individuano un punto che cade precisamente nella zona ove più sensibili furono gli effetti dinamici. Tale epicentro è assai prossimo a quello del violento terremoto verificatosi alle $23^{\mathrm{ln}} 30^{\mathrm{m}}$ del 3 febbraio 1949 , per il quale gravi danni ebbero a soffrire gli abitati di Paularo e delle sue frazioni di Casaso e Villamezzo.

Tutti gli Osservatorî dell'Alta Italia registrarono la scossa del 5 novembre 1956 e tracce di registrazione si ebbero anche in alcuni Osservatori dell'Italia centrale e meridionale. L'ora origine del sisma, dedotta da tali registrazioni, è $20^{\mathrm{h}} 45^{\mathrm{m}} 20^{\mathrm{s}}$.

Durante il 1956 non si ebbero sensibili repliche. Altre scosse abbastanza forti si verificarono nella stessa zona durante il febbraio del 1957. Di esse si dirà nelle pagine seguenti.

70. 25 novembre Macchiagodena (Campobasso)

Negli ultimi giorni di novembre e nei primi di dicembre del 1956, un periodo sismico piuttosto intenso ebbe luogo in provincia di Campobasso, nella zona a nord dei Monti del Matese. S'iniziò alle $16^{\mathrm{h}} 55^{\mathrm{m}}$ circa del 23 novembre con una scossa avvertita di IV-V grado nel paese di Macchiagodena e con intensità minore nei paesi vicini

Il 25 novembre, alle $03^{1} 06^{\mathrm{m}}$ circa, si verificò la scossa più intensa del periodo sismico. L'epicentro, con molta probabilità, fu nei pressi di Macchiagodena e ad esso possono approssimativamente essere assegnati, come coordinate geografiche, i valori $41^{\circ} 35^{\prime} \mathrm{N}$ e $14^{\circ} 24^{\prime} \mathrm{E}$. La maggiore intensità (VI grado) si manifestò appunto nel paese di Macchiagodena ove tutta la popolazione, svegliata all'improvviso dal forte movimento sismico, si riversò spaventata all'aperto. Caddero calcinacci in abbon- 
dante quantità e lesioni, in generale leggiere, si produssero in numerosi edifici. La scossa fu accompagnata e seguita da forti rombi. Fu riferito, e ciò non solo dagli abitanti di Macchiagodena ma anche di altre località vicine, che gli animali dettero segni di allarme qualche istante prima che la scossa fosse avvertita dalle persone.

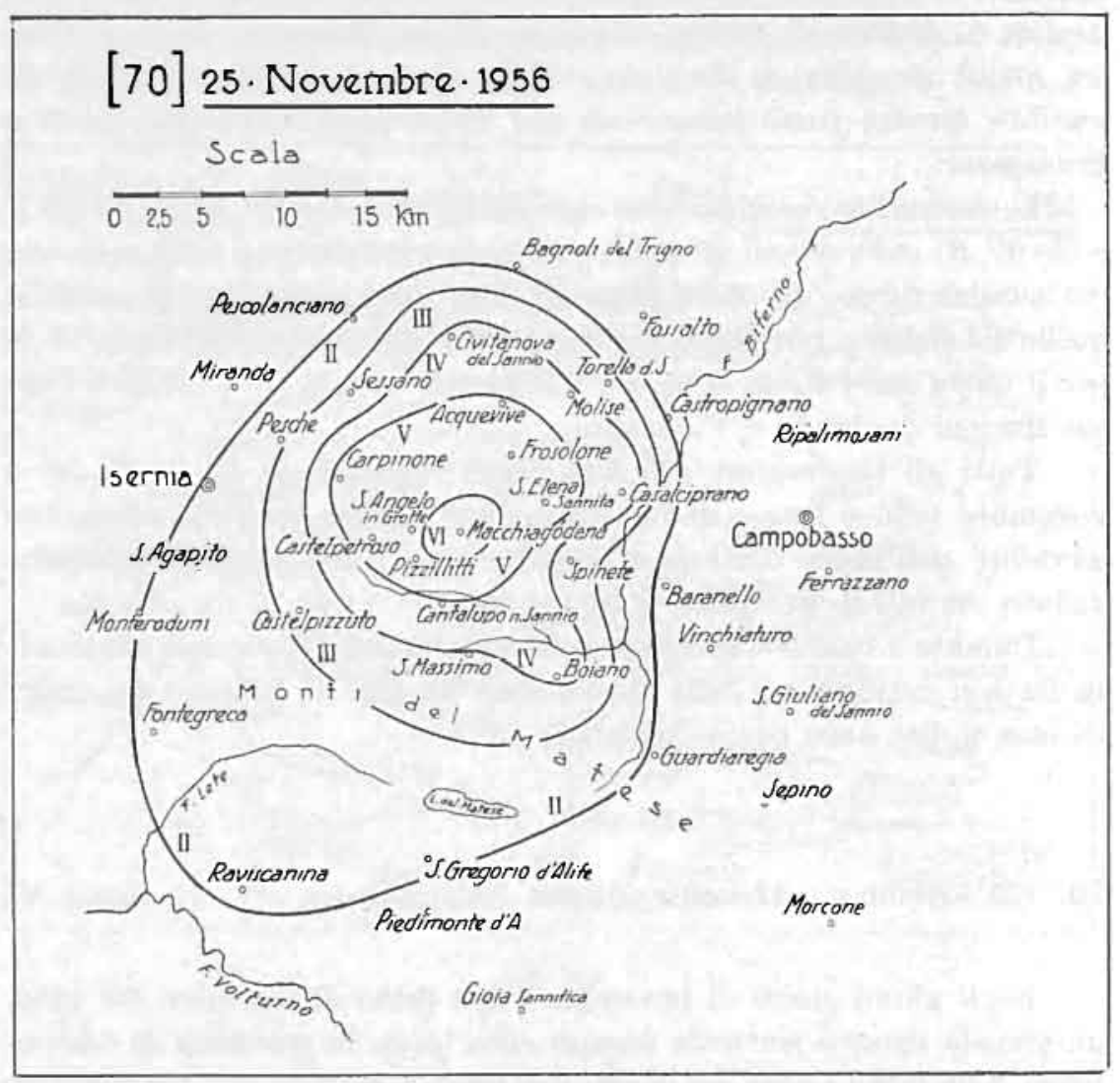

Fig. 44

Con intensità molto forte, come si è detto, la scossa si manifestò solo a Macchiagodena e nel territorio immediatamente eircostante. Fu poi forte nei comuni di Sant'Elena Sannita, Frosolone, Carpinone, Sant'Angelo in Grotte e Castelpetroso; mediocre in numerose altre località, fra cui Civitanova del Sannio, Casaleiprano e Boiano.

La propagazione macrosismica, come appare dalla figura 44 , fu piuttosto limitata. Il movimento tellurico si estese specialmente in dire- 
zione SW giungendo con leggerissima intensità in alcuni paesi, come Fontegreca e Raviscanina, situati alle pendici sud-occidentali dei Monti del Matese.

La scossa fu registrata negli Osservatori dell'Italia centrale. L'ora origine, dedotta dalle registrazioni, fu $03^{\text {h }} 06^{\mathrm{m}} 40^{\mathrm{s}}$. Numerose repliche di minore intensità si ebbero nei giorni successivi. Delle più notevoli si dirà nelle pagine seguenti.

\section{26 novembre \\ Amandola (Ascoli P.) \\ Caldarola (Macerata) \\ Grado V}

Nel mese di novembre del 1956, alcune scosse telluriche d'intensità non molto forte ebbero luogo nell'Appennino Marchigiano, in quella stessa zona ove pochi anni avanti, il $1^{\circ}$ settembre 1951 , un fortissimo terremoto si era abbattuto causando danni assai gravi negli edifici di numerose cittadine e paesi.

La prima scossa, leggera, avvenne verso le $03^{\text {h }}$ del giorno 13 e fu avvertita nei comuni di Sarnano, Mogliano, Caldarola e Bolognola, tutti della provincia di Macerata. Anche nella città di Macerata il movimento tellurico venne avvertito, ma molto leggermente e da poche persone in relazione al numero degli abitanti.

La seconda scossa, avvenuta alle $04^{\text {h }} 53^{\mathrm{m}}$ circa del giorno 26 , fu di forte intensità e venne sentita di $\mathrm{V}$ grado sopra un territorio comprendente nel suo interno i comuni di Caldarola, di Bolognola e di Amandola. In questa zona il movimento ebbe carattere sussultorio e fu accompagnato da rombi.

La isosista di IV grado, di forma irregolarissima (fig. 45), si estende, fra le valli dei fiumi Esino ed Aso, principalmente nelle province di Macerata e di Ascoli e con alcune sue propaggini penetra nella provincia di Teramo includendo il comune di Civitella del Tronto e nella provincia di Perugia includendo i comuni di Preci e di Gualdo Tadino. La fig. 45 ci dispensa dall'enumerare i molti altri luoghi ove la scossa fu avvertita con sensibile intensità.

Un andamento simile a quella di IV grado presenta la isosista di III grado. Nella sua parte orientale però, fra le valli dei fiumi Potenza ed Aso, essa si espande verso la costa adriatica includendo i comuni di Mogliano e di Montegiorgio.

Una terza scossa infine, di mediocre intensità, si verificò verso le $04^{\mathrm{h}} 30^{\mathrm{m}}$ del 28 novembre. Fu avvertita, sembra, soltanto in provincia 
di Macerata: di IV grado a Sefro e a San Severino Marche, di III a Gualdo e a Tolentino. Non si ebbero notizie da altre località.

La seconda delle scosse sopra ricordate fu registrata a Roma e in alcuni altri Osservatori dell'Italla centrale.

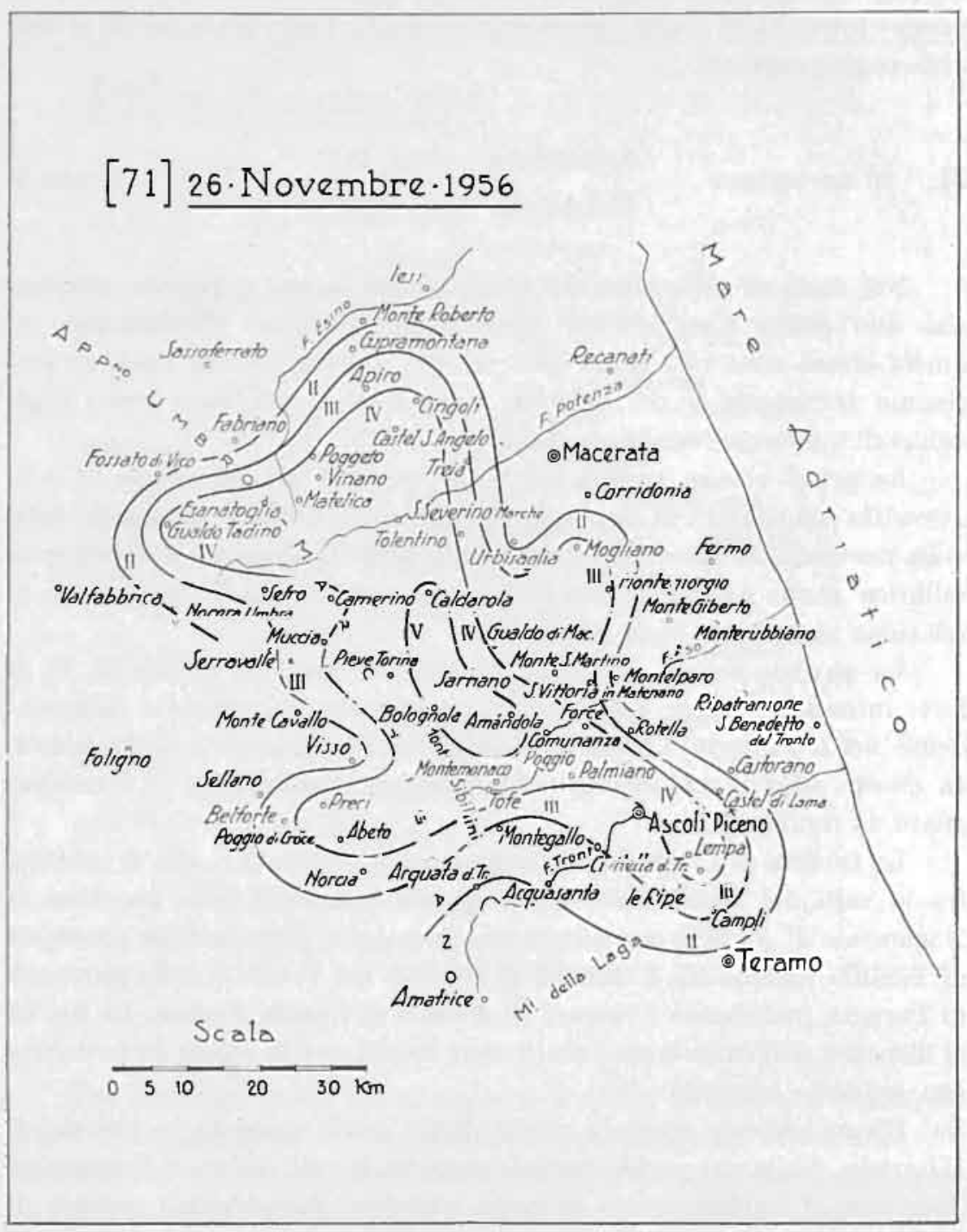

Fig. 45 
72. 29 novembre

S. Angelo in Grotte (Campobasso) Macchiagodena ( ” )

Grado V

La più notevole fra le numerose repliche che seguirono la scossa del 25 novembre 1956 (n. 70) fu quella avvenuta, nello stesso mese, alle $03^{\mathrm{h}} 15^{\mathrm{m}}$ circa del giorno 29 , la quale manifestò la sua maggiore intensità (V grado) a Sant'Angelo in Grotte e a Macchiagodena, paesi distanti l'uno dall'altro poco più di tre chilometri in linea d'aria. Verso nord-ovest e nord est essa si propagò un po' più sensibilmente della scossa del 25 novembre: risultò infatti di IV grado nei paesi di Sessano e Torella del Sannio, nei quali la scossa del 25 novembre era stata solo leggermente avvertita. Di IV grado risultò anche a Frosolone e nella sua frazione di S. Pietro in Valle, a Sant'Elena Sannita, a Molise e a Casalciprano. Con intensità leggera o leggerissima fu percepita nella città d'Isernia e nei paesi di Bagnoli del Trigno, Sant'Agapito, Fontegreca e Raviscanina.

Un'altra scossa meritevole di menzione si verifico il 2 dicembre 1956 alle $09^{\mathrm{h}} 35^{\mathrm{m}}$. E da supporre però che l'epicentro sia stato questa volta fra S. Angelo in Grotte e Sessano, a qualche chilometro di distanza da quello relativo alla scossa del 25 novembre. Sembra infatti che nelle predette due località $\mathrm{e}$ in Carpinone il movimento tellurico sia stato avvertito con intensità maggiore (IV-V grado) che negli altri paesi della zona. Di IV grado risultò a Macchiagodena, a Civitanova del Sannio, a Sant'Elena Sannita, a Casalciprano ed a Pesche; di II grado a Frosolone e ad Acquevive. 


\section{Anno 1957}

73. 25 gennaio

Pizzoli (L'Aquila)

Grado V-VI

La mattina del 25 gennaio 1957 , alle $05^{\mathrm{h}} 40^{\mathrm{m}}$ circa, le popolazioni di numerosi paesi delle province dell'Aquila e di Teramo furono destate da una sensibile scossa di terremoto preceduta ed accompagnata, in alcune località, da intensi boati.

La maggiore intensità della scossa, valutabile fra il V ed il VI grado, fu accertata in una ristretta zona situata a NW della città dell'Aquila e comprendente i paesi di Pizzoli, di Marruci (frazione di Pizzoli) e di Barete. In tale zona, ove con molta probabilità può essere posto l'epicentro, la scossa venne sentita da tutti gli abitanti, gran parte dei quali, in preda allo spavento, abbandonarono le ease accampandosi nella eampagna circostante. Non si ebbero peró danni di rilievo; solo si aggravarono alcune lesioni già esistenti in qualche edificio vecchio e di difettosa costruzione.

Il movimento tellurico fu sensibilmente avvertito su di un'area assai estesa in confronto alla limitatezza della zona epicentrale. Risultò infatti di IV grado nei comuni di Montereale, Capitignano, Cagnano Amiterno, Tornimparte, Rocca di Mezzo, Caporciano ed Ofena, tutti in provincia dell'Aquila; nella città dell'Aquila e nelle sue numerose frazioni; nei comuni di Isola del Gran Sasso d'Italia, di Tossicia e di Montorio al Vomano, in provincia di Teramo. Tale area pertanto, come appare dalla figura 46, si estende lungo la Valle del Fiume Aterno, abbraccia una parte dell'altopiano di Rocca di Mezzo e, attraverso il Gran Sasso d'Italia, si protende in provincia di Teramo, oltre le valli dei Fiumi Vomano e Mavone.

La scossa si propagò anche nei Monti della Laga, giungendo con leggera intensità nel territorio dei comuni di Rocca S. Maria e di Valle Castellana. Leggermente avvertita fu anche a Pietracamela (Teramo), a Cittareale, Borbona e Rocca di Corno, in provincia di Rieti; ad Ovindoli, Rovere, Navelli e Capestrano, in provincia dell'Aquila.

Leggerissima fu percepita nella città di Teramo.

Il sisma fu registrato negli Osservatorî dell'Aquila, di Collurania (Teramo) e di Roma. 


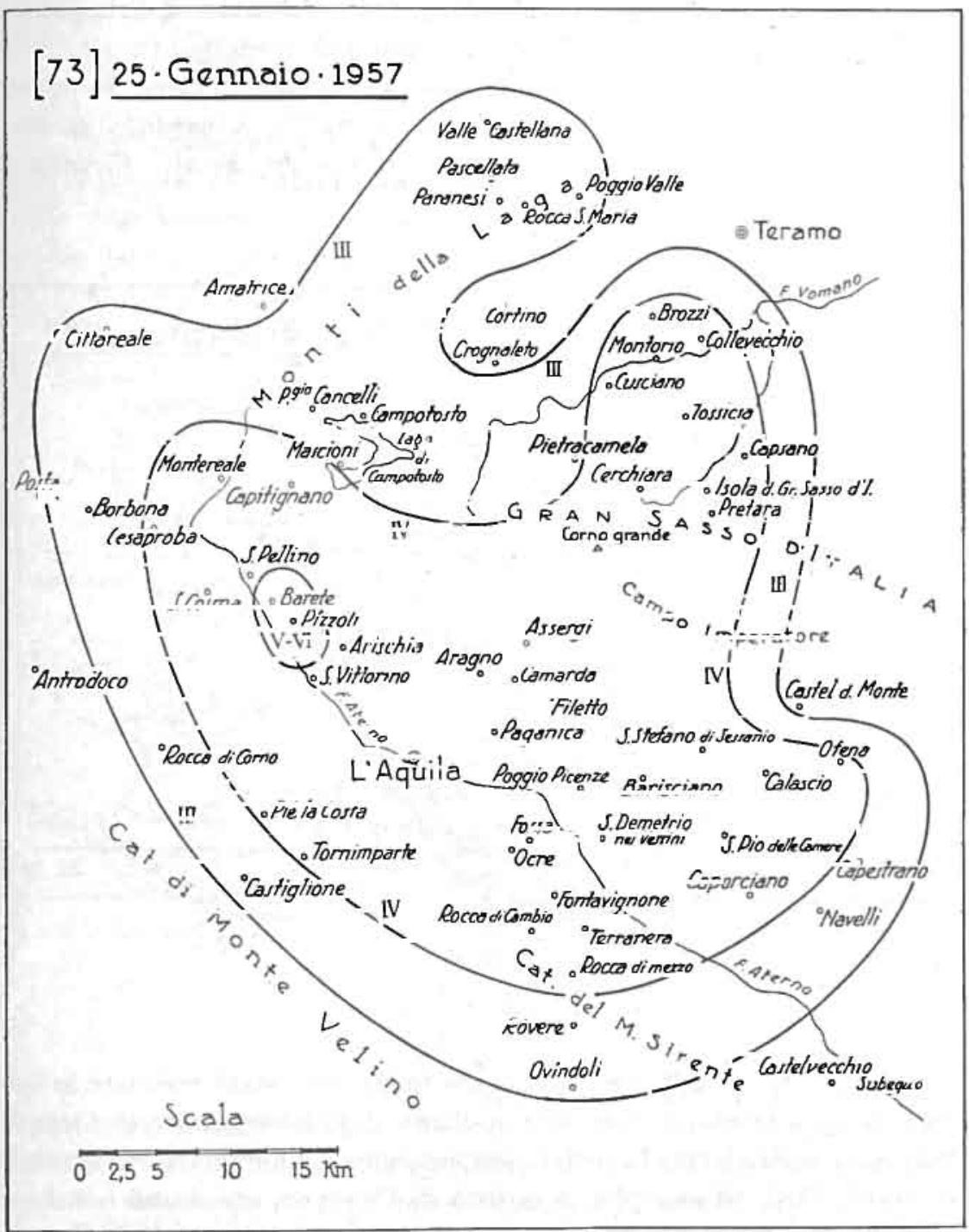

Fig. 46

74. 10 febbraio

Tolmezzo (Udine)

Grado V

Il 10 febbraio 1957 , alle $01^{\mathrm{H}} 15^{\mathrm{m}}$ circa, nei comuni di Tolmezzo e di Arta, accompagnata da un forte rombo, si verifico una scossa di terremoto della durata di $3-4$ secondi e di un'intensità giudicata pari al V 
grado Mercalli. L'epicentro fu probabilmente tra Tolmezzo ed Arta poichè solo in tali localltà il sisma raggiunse l'intensità predetta.

A Paularo, Ligosullo, Villa Santina, Verzegnis e Andreis la scossa fu avvertita di IV grado; di III a Paluzza, Sutrio, Ravascletto, S. Stefano di Cadore, Spilimbergo, Artegna, Gemona del Friuli, Resiutta e Tarvisio.

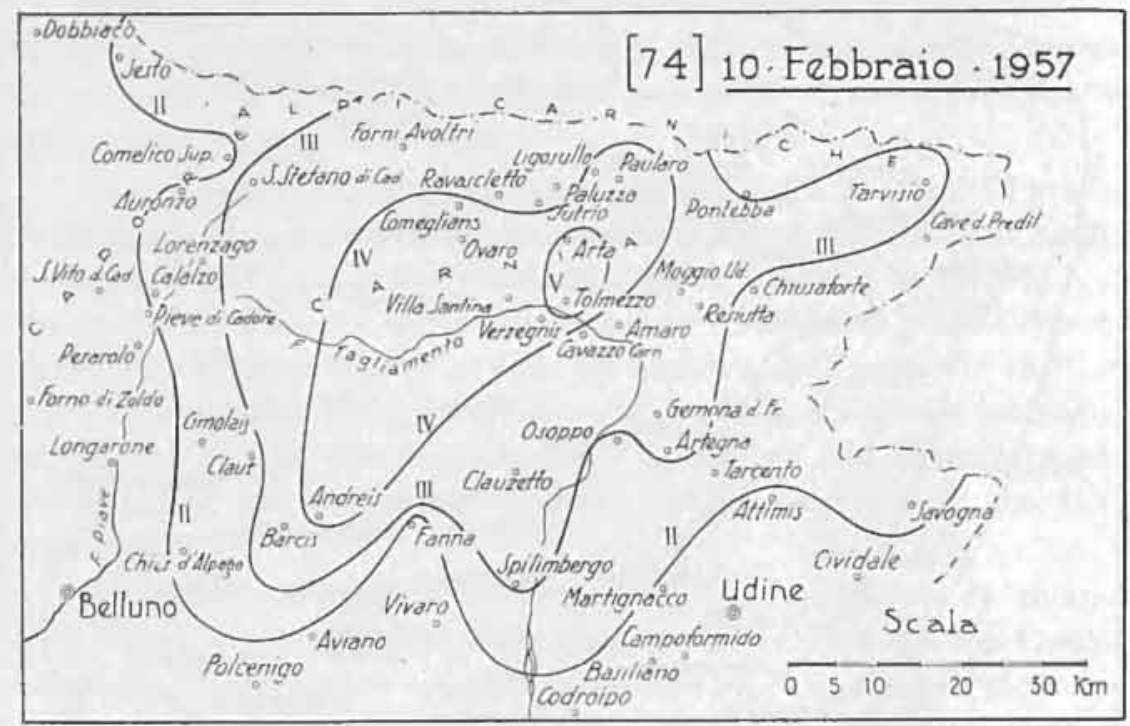

Fig. 47

Come appare dalla figura 47 , nella quale sono state tracciate le isosiste limitatamente al territorio italiano, l'estensione macrosismica fu notevole. Assai diversa fu però la propagazione del movimento nelle varie direzioni. Cosi, ad esempio, la isosista di IV grado, che a sud e sud-est di Tolmezzo risulta molto ravvicinata alla zona epicentrale indicando una brusca diminuzione d'intensità, si protende invece ampiamente in direzione sud-ovest, fin oltre il territorio del comune di Andreis.

La regione itallana ove la scossa si rese percettibile all'uomo ha ai suoi limiti i paesi di Sesto, in provincia di Bolzano; di Auronzo, Calalzo e Chies d'Alpago, in provincia di Belluno; di Cimolais, Fanna, Tarcento e Savogna, in provincia di Udine. 
Dopo pochi giorni dalla scossa di cui sopra si è detto, e precisamente alle $8^{\mathrm{n}} 52^{\mathrm{m}}$ del 18 febbraio, un altro forte movimento tellurico ebbe luogo nelle Alpi Carniche. Il suo epicentro fu certamente assai prossimo a quello del terremoto del o novembre 1956 (n. 69). La maggiore intensità ( $V$ grado) si manifesto infatti in una ristretta zona comprendente i soli

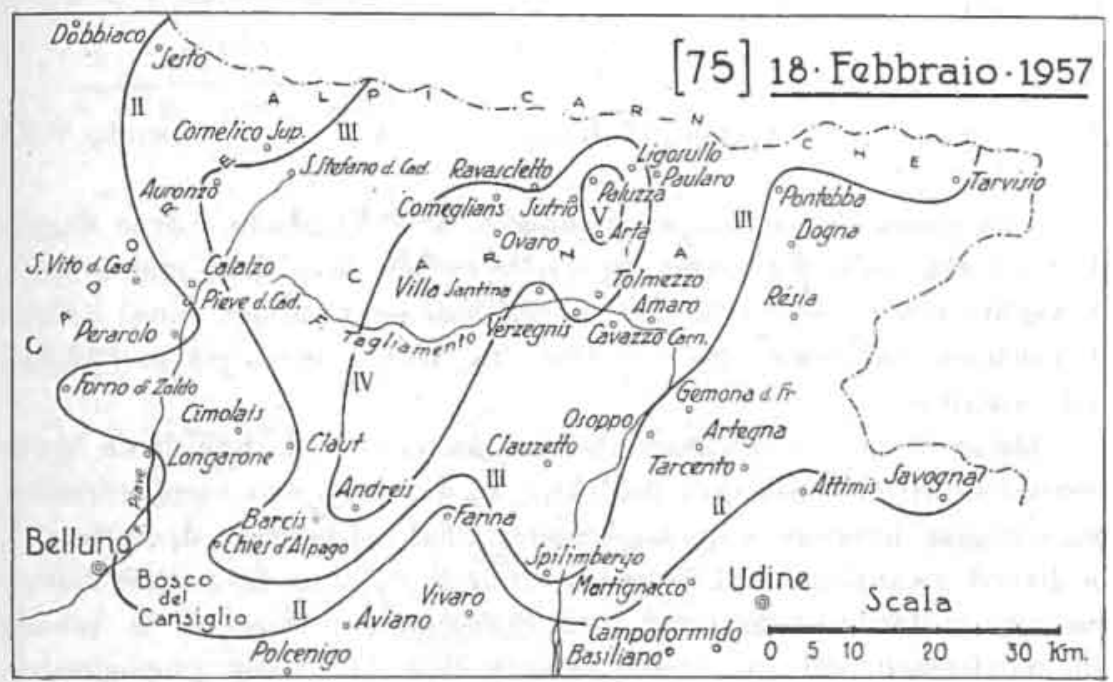

Fig. 48

comuni di Paluzza e di Arta. In queste località il movimento tellurico, accompagnato da intensi rombi, venne sentito con carattere prevalentemente sussultorio ed ebbe una durata di 3-4 secondi.

Con sensibile intensità (IV grado), la scossa fu avvertita in una zona piuttosto ampia estendentesi principalmente in direzione sud-ovest (come la zona isosismica di pari grado del terremoto del 10 febbraio) e racchiudente nel suo interno i comuni di Ligosullo, Sutrio, Comeglians, Tolmezzo ed Andreis.

La propagazione macrosismica fu all'incirca uguale a quella della scossa avvenuta il 10 febbraio 1957 (v. fig. 48). 
Un movimento tellurico sussultorio di forte intensità e della durata di circa 5 secondi, si verificò a $S$. Giovanni Rotondo verso le $8^{\mathrm{h}} 40^{\mathrm{m}}$ del 23 febbraio 1957. Non provocò alcun danno agli edifici, ma fu avvertito da tutti con grande spavento nelle case e da moltissimi anche nelle strade.

La scossa, che fu accompagnata da un forte boato, ebbe però una propagazione limitatissima; passò infatti quasi inosservata nei paesi circostanti.

Una scossa di terremoto, sussultoria, di V-VI grado e della durata di 4 secondi, colpi Zafferana Etnea alle $10^{\mathrm{h}} 26^{\mathrm{m}}$ circa del 3 marzo 1957. Avvertita con spavento da tutti gli abitanti del Comune, causò caduta di calcinacci in alcune case e il crollo di muri a seceo, già pericolanti, nelle campagne.

Questa scossa, verificatasi qualche giorno dopo la fine di un breve periodo di attività esplosiva dell'Etna, fu avvertita con varia intensità, ma ovunque inferiore a quella manifestatasi nel comune di Zafferana, in diversi paesi situati sui fianchi del grande vulcano. Le notizie macrosismiche pervenuteci non sono però sufficienti per tracciare le isosiste del movimento tellurico. Possiamo solo dire che la sua propagazione, come generalmente accade per i sismi di origine vulcanica, si verificò entro una superficie di estensione molto limitata. Esso fu sensibile a Giarre; leggero nella città di Catania e nei paesi di Maletto, Bronte, Belpasso e Carruba (frazione di Riposto); leggerissimo a Riposto, Fiumefreddo di Sicilia, Piedimonte Etneo, Tremestieri Etneo e Castiglione di Sicilia. Passó inavvertito a Randazzo, Biancavilla, S. Maria di Licodia, Camporotondo Etneo, Pedara e Trecastagni.

78. 8 marzo

Ragusa

Grado V

Verso le $03^{\mathrm{h}} 18^{\mathrm{m}}$ dell' 8 marzo 1957 , una scossa di terremoto abbastanza forte si verificò nella parte sud-orientale della Sicilia e venne particolarmente avvertita a sud dei Monti Iblei, in una zona di forma presso che ellittica con l'asse maggiore lungo la valle del Fiume Irminio. Tale zona, 
in cui probabilmente si trovò l'epicentro, comprende le città di Ragusa e di Modica ed i paesi di Chiaramonte Gulfi e Giarratana. In queste località la scossa fu valutata di V grado, ebbe una durata di 5-8 secondi e venne avvertita, con vivo allarme delle popolazioni, insieme a rombi piuttosto intensi.

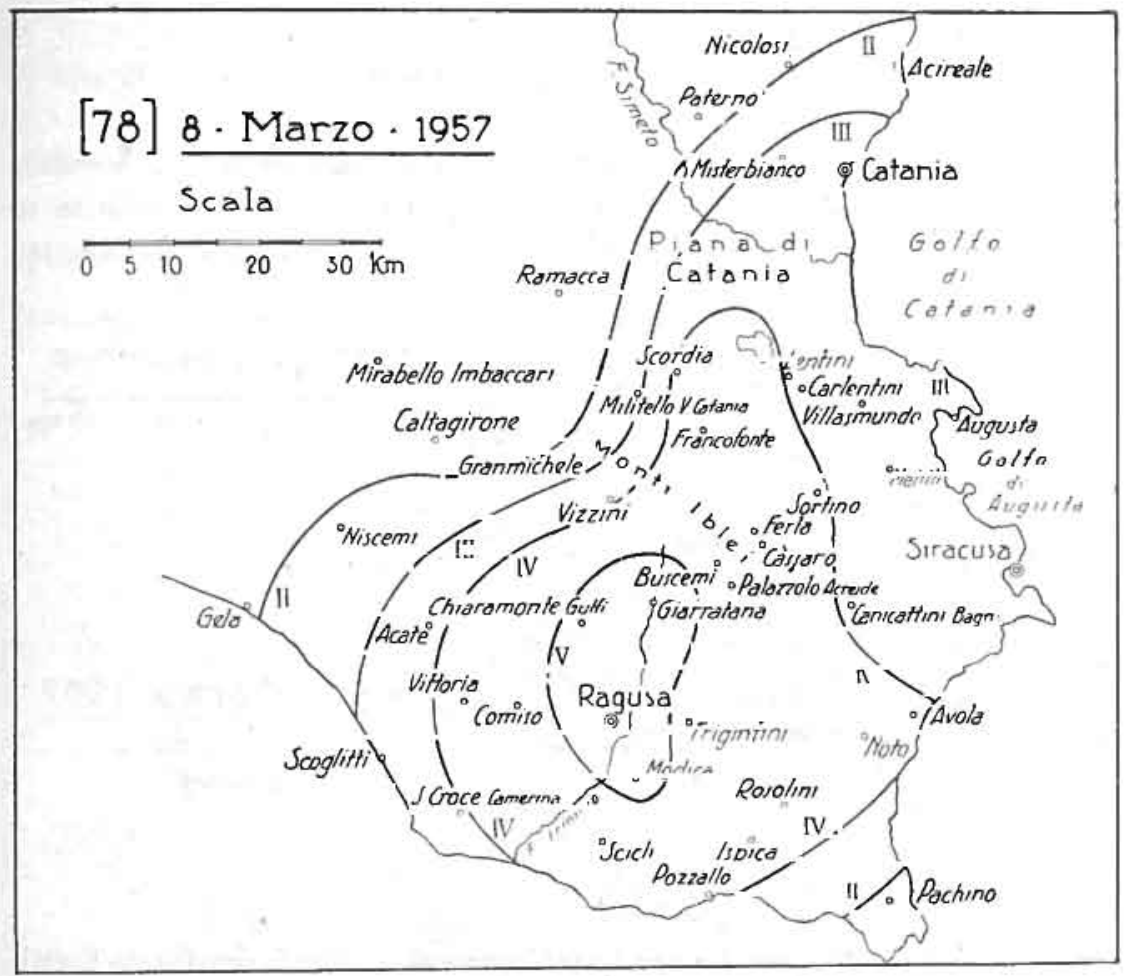

Fig. 49

La scossa risultò sensibile (v. fig. 49) a Vittoria, Comiso, Scicli, Pozzallo, Ispica, Frigintini, in provincia di Ragusa; a Buscemi, Sortino, Rosolini, Cassaro, Noto, Avola, Palazzolo Acreide, Francofonte, in provincia di Siracusa e a Scordia, in provincia di Catania. Nella città di Catania e in diverse località del Catanese fu avvertita leggermente; pure leggermente nella città di Siracusa e nei comuni di Augusta, Canicattini Bagni, Melilli, Lentini, Carlentini, tutti in provincia di Siracusa.

Con intensità leggerissima fu percepita a Niscemi (Caltanissetta), a Militello Val Catania e in alcuni centri delle pendici orientali e sud- 
orientali dell'Etna, come Giarre ed Acireale. Del tutto inosservata passò a Pachino e nell'estrema punta sud-orientale della Sicilia.

Venne registrata negli Osservatorî di Reggio Calabria e di Messina. L'ora origine dedotta dalle registrazioni fu $03^{\mathrm{h}} 17^{\mathrm{m}} 52^{\mathrm{s}}$.

Si ebbe una repllea nella stessa giornata alle $13^{\mathrm{h}} 25^{\mathrm{m}}$ circa.

79. 8 marzo

Sanremo - Taggia (Imperia)

Grado V

Una scossa tellurica ondulatoria di brevissima durata (1-2 secondi) $\mathrm{ma}$ abbastanza intensa ( $\mathrm{V}$ grado), il cui epicentro probabilmente fu in mare, venne avvertita, alle $5^{\mathrm{h}} 12^{\mathrm{m}}$ circa dell'8 marzo 1957 , a Sanremo

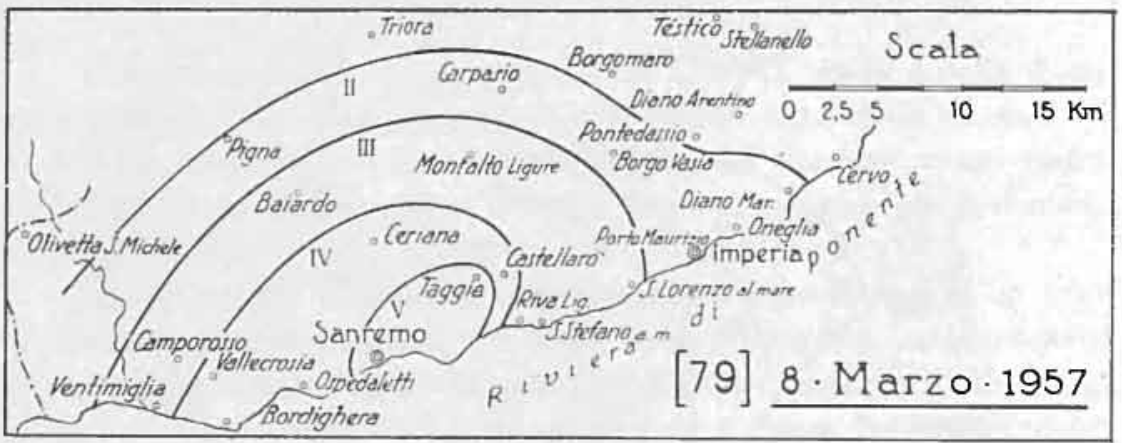

Fig. 50

e a Taggia. Sulla Riviera Ligure fu percepita con varia intensità da Ventimiglia a Diano Marina: di IV grado a Bordighera e ad Ospedaletti; di III a Ventimiglia, a Riva Ligure, a S. Stefano e a S. Lorenzo al Mare; di II ad Imperia e a Diano Marina.

$\mathrm{Nel}$ retroterra il movimento tellurico fu avvertito sensibilmente a Vallecrosia, a Ceriana e a Castellaro; leggermente a Camporosso, Baiardo e Montalto Ligure.

Dalle isosiste della fig. 50 si rileva la modesta estensione macrosismica della scossa.

Due leggere scosse premonitorie erano state avvertite, a Sanremo ed in altre localltà della Riviera di Ponente, pochi minuti prima della scossa delle $5^{\mathrm{h}} 12^{\mathrm{m}}$. 
80. 12 marzo

Norcia (Perugia)

Grado VI

Una scossa molto forte di terremoto si verifico il 12 marzo 1957, alle $17^{\mathrm{h}} 20^{\mathrm{m}}$ circa, nella pianura umbra di S. Scolastica, a sud-ovest dei Monti Sibillini, raggiungendo la sua maggiore intensità (VI grado) nella

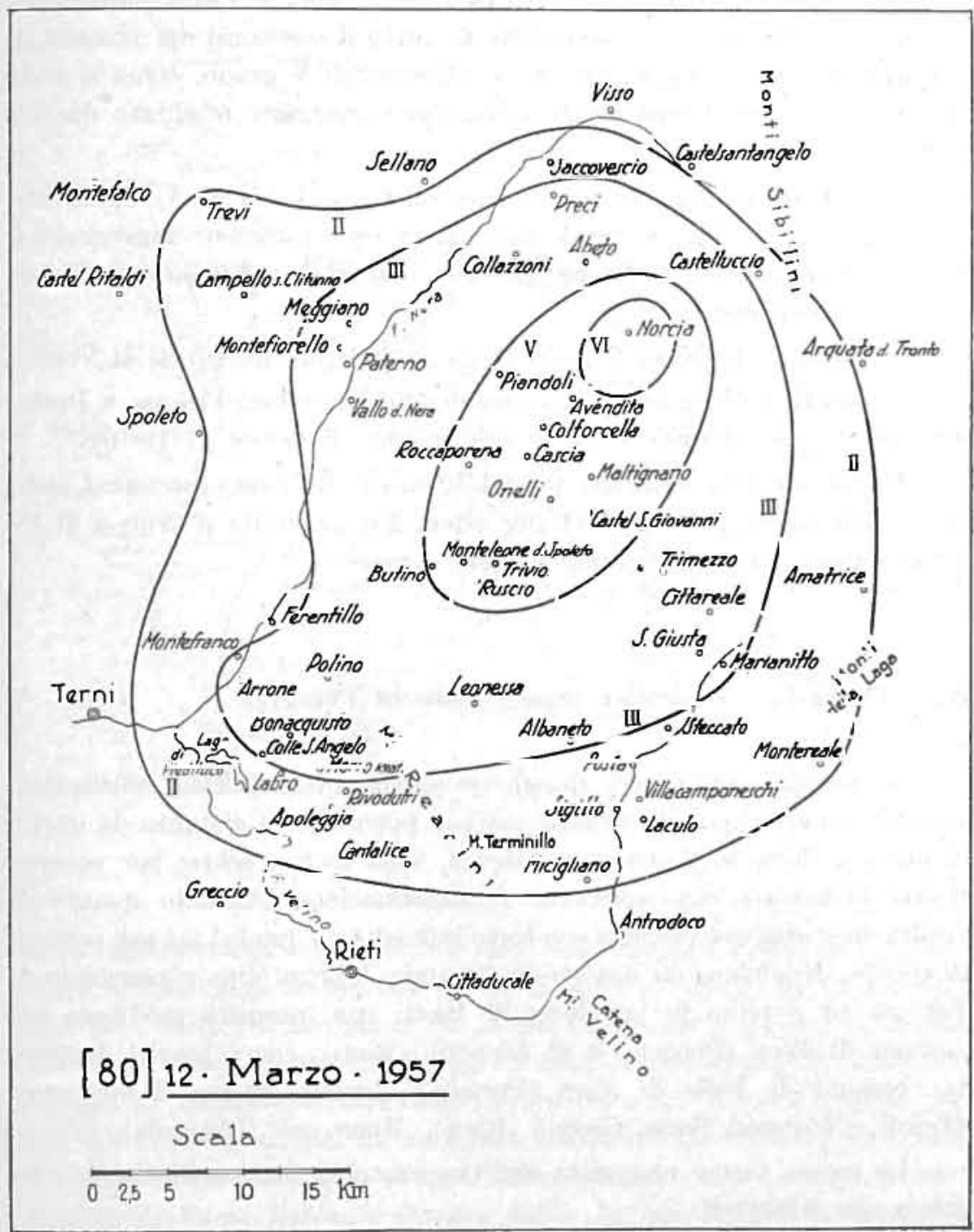

Fig. 51 
cittadina di Norcia e nel territorio circostante. Ivi il movimento tellurico, sussultorio ed accompagnato da rombi, fu avvertito da tutti, sia all'aperto che nell'interno delle case, e causó la caduta di calcinacci e leggiere fenditure in numerosi edifici.

La propagazione macrosismica fu alquanto limitata. Verso nord e verso est il movimento decrebbe rapidamente d'intensità, sì da passare completamente inosservato ad Arquata del Tronto, a Castel-Santangelo, a Visso e a Sellano. Fu invece forte in tutto il territorio dei comuni di Cascia e di Monteleone di Spoleto. La isosista di V grado, come si vede in fig. 51, ha una forma ellittica con l'asse maggiore orientato da NE a SW.

L'intensità fu leggiera nei comuni di Preci, Vallo di Nera, Montefranco, Arrone, Polino, Morro Reatino, Leonessa e Cittareale; leggerissima, a Trevi, Campello sul Clitunno, Cantalice, Rivodutri, Micigliano, Montereale, Amatrice, ece.

L'epicentro del sisma fu, con molta probabilità, nei pressi di Norcia.

Si ebbero registrazioni negli Osservatori di Roma, Firenze e Prato. Da esse si potè desumere, come ora origine, il valore $17^{\mathrm{h}} 19^{\mathrm{m}} 18^{\mathrm{s}}$.

Un'altra scossa, originata probabilmente dallo stesso ipocentro, ebbe luogo il 5 aprile 1957 alle $03^{\mathrm{h}} 20^{\mathrm{m}}$ circa. Fu avvertita a Norcia di IV grado ed ebbe una propagazione limitatissima.

81. 13 marzo Amatrice (Rieti) - Cascia (Perugia)

Le notizie pervenuteci su questo movimento tellurico, verificatosi nella prima mattina del 13 marzo 1957, a poche ore di distanza da quello di cui si è detto al numero precedente, sono troppo scarse per poterne fissare le isosiste con sufficiente approssimazione. Secondo quanto ci risulta, la scossa fu avvertita con forte intensità ( $\mathrm{V}$ grado) nei soli comuni di Cascia, Monteleone di Spoleto ed Amatrice, i primi due in provincia di Perugia ed il terzo in provincia di Rieti; con intensità mediocre nei comuni di Preci (Perugia) e di Leonessa (Rieti); con intensità leggera nei comuni di Vallo di Nera (Perugia), Arrone, Polino, Montefranco (Terni), Cittareale, Posta, Greccio (Rieti), Montereale (L'Aquila).

La scossa venne registrata nell'Osservatorio sismico di Roma, con inizio alle $04^{\mathrm{h}} 19^{\mathrm{m}} 06^{\mathrm{s}}$. 


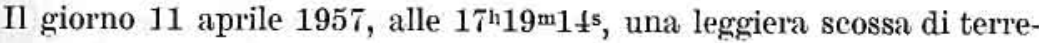
moto fu avvertita a Roma da numerose persone, specialmente nei quartieri situati nella zona nord-orientale della città, quali il Nomentano, il Macao, il Tufello e Montesacro. L'epicentro, determinato in base alle

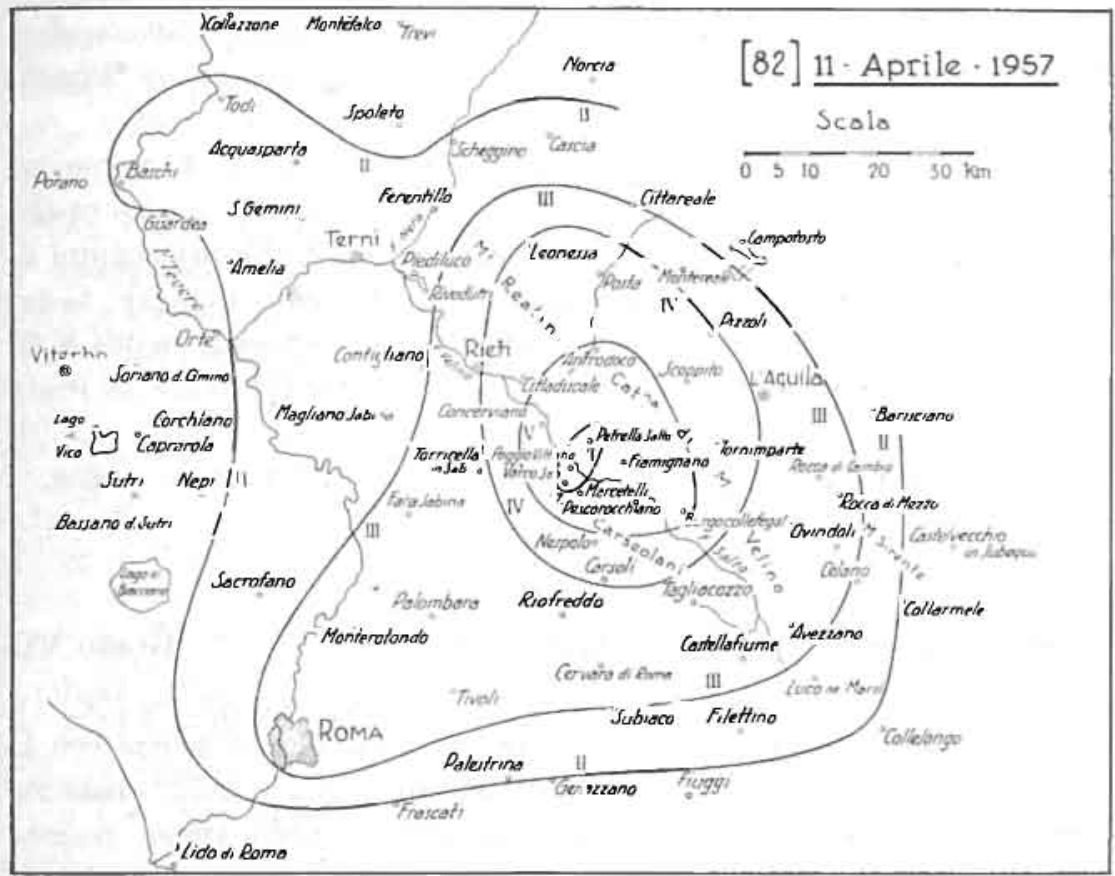

Fig. 52

registrazioni sismografiche ottenute nell'Osservatorio di Roma dell'I.N.G., risultò nel punto di coordinate $42^{\circ} 17^{\prime} \mathrm{N}$ e $13^{\circ} 02^{\prime} \mathrm{E}$, e cioè nella valle del Fiume Salto fra la Catena dei Monti Carseolani e quella del Monte Velino, ad una distanza da Roma di circa 60 chilometri in direzione nord-est. Le notizie macrosismiche successivamente pervenute confermavano tale determinazione. La maggiore intensità della scossa si era infatti avuta in una ristretta zona situata nella Valle Cicolana e comprendente i comuni di Varco Sabino e Petrella Salto. In tali località la scossa fu valutata di VI grado essendo stata da tutti moIto fortemente sentita 
ed avendo causato molte fenditure in case di vecchia costruzione. In nessun'altra località si ebbero a lamentare danni di sorta.

La fig. 52 mostra le linee isosiste tracciate in base alle notizie macrosismiche a nostra disposizione. La zona di forte intensità si estende tutta in provincia di Rieti ed abbraccia per intero la subregione nota con il nome di "Cicolano ». La scossa fu infatti sentita di V grado nei comuni di Marcetelli, Pescorocchiano, Borgocollefegato, Fiamignano, Concerviano ed Antrodoco. La zona isosismica di IV grado si estende in parte nella provincia di Rieti, con i comuni di Leonessa, Posta, Cittaducale e Nespolo, ed in parte in provincia dell'Aquila con i comuni di Pizzoli, Scoppito, Tornimparte e Carsoli.

La scossa fu avvertita inoltre leggermente a Rieti, a L'Aquila ed anche, come si è detto, nella città di Roma. Verso nord-ovest si propagò anche in Umbria giungendo con leggerissima intensità fin nei comuni di Baschi e di Todi. Sotto forma microsismica il movimento tellurico, la cui " magnitudo" calcolata in base ai sismogrammi ottenuti nell'I.N.G. risultò di 4,1, venne registrato anche negli Osservatorî di Firenze, di Prato e di Messina.

Una lieve replica si verificò alle $18^{\mathrm{h}} 50^{\mathrm{m}}$ circa dello stesso giorno.

Non erano trascorsi neanche cinque mesi dall'ultima scossa con la quale si era chiuso il lungo periodo sismico svoltosi nell'Appennino Tosco-Emiliano durante il 1956, quando s'iniziò nella stessa regione un'altra serie di movimenti tellurici, alcuni dei quali di grande violenza.

Il nuovo periodo sismico si aprì con una breve scossa di mediocre intensità avvertita nell'alta valle del Rabbi, specialmente nel comune di Premilcuore, alle $16^{\mathrm{h}} 40^{\mathrm{m}}$ eirca del 15 aprile 1957.

Il giorno 17 , alle $03^{\mathrm{h}} 22^{\mathrm{m}} 36^{\mathrm{s}}$, si verificò il prino massimo del periodo sismico. Una violenta scossa, valutabile di VII grado, si abbattè su Santa Sofia, sulle sue numerose frazioni, tra cui Cabelli, Corniolo, Spinello, e su Monteguidi, frazione del comune di Bagno di Romagna. Tutta la popolazione di questi centri, destata all'improvviso dal fortissimo movimento tellurico, abbandonò in preda al panico le abitazioni trascorrendo la notte all'aperto. Sensibili furono i danni negli edifici: caddero comignoli e tegole, vetri andarono in frantumi, numerose e serie lesioni si produssero nelle murature, particolarmente gravi in quelle dei fabbricati rurali. 
La scossa, avvertita nelle predette località con carattere ondulatorio, ebbe una durata di circa cinque secondi e fu preceduta da un intenso boato. Per il suo epicentro, in base alle registrazioni ottenute negli Osservatorî di Roma, Pavia, Bologna e Firenze, furono determinate nell'I.N.G. le coordinate $43^{\circ} 54^{\prime} \mathrm{N}$ e $11^{\circ} 50^{\prime} \mathrm{E}$, le quali individuano un punto situato nella valle dell'Alto Bidente, qualche chilometro ad est del paesino di Corniolo.

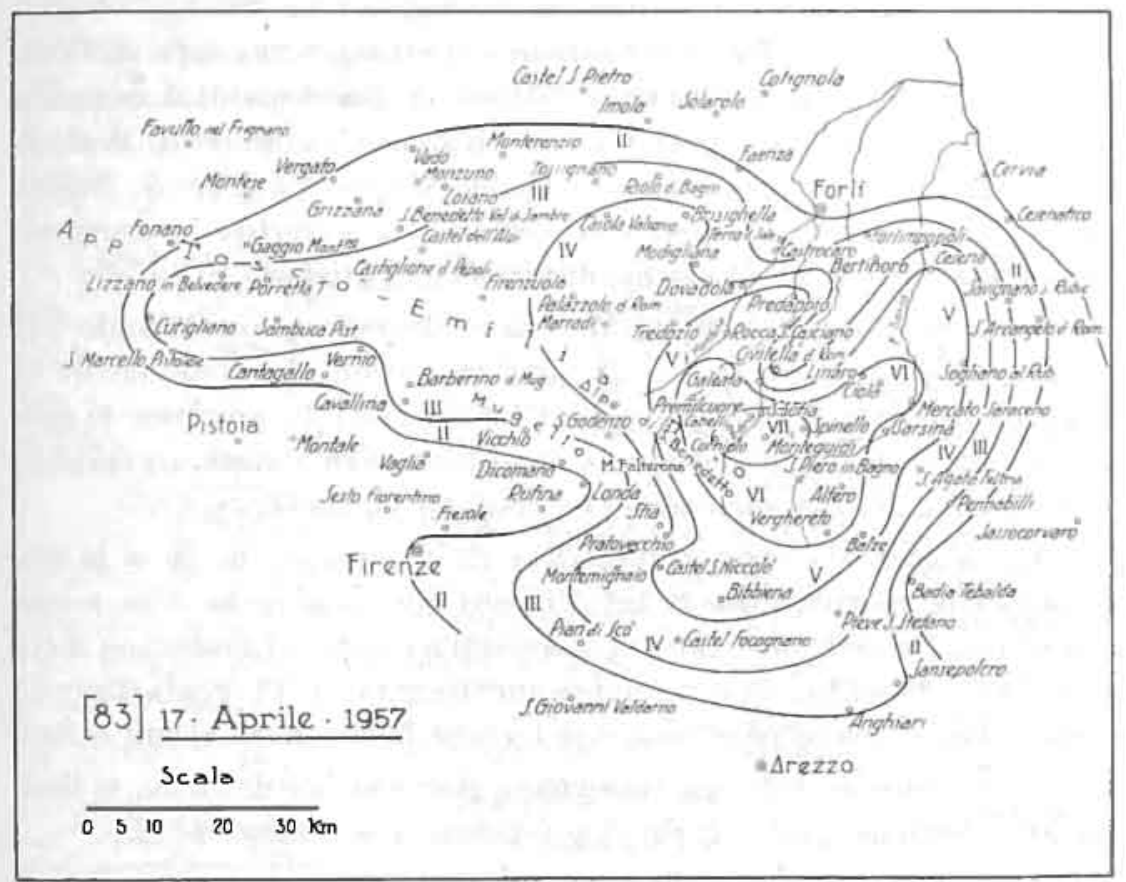

Fig. 53

La zona isosismica molto forte (VI grado), ove la scossa venne avvertita da tutti con grande spavento e si verificarono fenditure leggiere in case di vecchia costruzione, distacchi d'intonaco e crollo di qualche comignolo, fu alquanto vasta. Essa si estese infatti dall'Alta Valle del Montone alla Valle del Savio includendo i territori dei comuni di Premilcuore, Verghereto, Bagno di Romagna e Mercato Saraceno, tutti in provincia di Forli.

È interessante, dal punto di vista macrosismico, la brusca diminuzione d'intensità subita dal movimento tellurico lungo la Valle del Bidente verso Galeata e Civitella di Romagna, località che distano da 
Santa Sofia 5 e 7 chilometri circa rispettivamente. Nella prima, la scossa fu sentita appena di V grado; nella seconda, solo di III. Notevolmente, invece, la scossa si propagó lungo tutta la Valle del Savio e lungo buona parte della Valle del Rabbi, cosi da giungere con intensità ancora forte nella città di Cesena e nel territorio del comune di Predappio.

Anche in altre direzioni, come appare dalla fig. 53, la propagazione energetica della scossa presentò sensibili discontinuità.

Oltre che nei comuni di Cesena e di Predappio, il movimento tellurico fu forte nei comuni di Rocca S. Casciano e Sarsina, in provincia di Forli, ed in quelli di Castel $S$. Niccolò e Bibbiena in provincia di Arezzo. Fu sensibile a Brisighella e Casola Valsenio (Ravenna); a Marradi, Tredozio e Dovadola (Forli); a Montemignaio, Castel Focognano e Pieve S. Stefano (Arezzo). Con intensità leggera e leggerissima fu avvertito in numerosi comuni delle province di Bologna, di Pistoia e di Firenze.

Poche ore dopo, alle $09^{\mathrm{h}} 40^{\mathrm{m}} 37^{\mathrm{s}}$, una fortissima replica, di grado VII come la scossa precedente, ma di carattere sussultorio, colpì ancora la cittadina di Santa Sofia ed i paesi ad essa circostanti, portando lo sgomento nelle popolazioni ancora attendate fuori degli abitati, aggravando notevolmente le lesioni agli edifici e eausandone delle nuove.

Anche questa scossa fu preceduta da un intenso boato e la sua propagazione macrosismica fu press'a poco uguale a quella della scossa precedente. Fu peró meno intensa a Mercato Saraceno ed invece più forte a Rocca S. Casciano, ove raggiunse un'intensità di VI grado facendo crollare alcuni comignoli e causando leggiere fenditure in alcuni edifici.

Delle numerose repliche, susseguitesi fino alla fine di aprile, ci limitiamo a ricordare quelle di cui si son potute avere sicure notizie.

Il 17 aprile si ebbero: una scossa alle $10^{\mathrm{h}} 30^{\mathrm{m}}$ circa avvertita di IV-V grado a Santa Sofia e di IV a Premilcuore; una scossa alle $17^{\mathrm{h}} 50^{\mathrm{m}}$ circa, avvertita di IV grado a Santa Sofia, a Galeata e a Premilcuore; una scossa alle $18^{\mathrm{h}} 10^{\mathrm{m}}$ circa, avvertita di IV grado a Santa Sofia.

Il 18 aprile, alle $7^{\mathrm{h}} 30^{\mathrm{m}}$ ed alle $12^{\mathrm{h}} 30^{\mathrm{m}}$ rispettivamente, furono avvertite a Santa Sofia due scosse :la prima di III e la seconda di IV grado.

Il 19 aprile due scosse di IV grado si ebbero ancora a Santa Sofia alle $4^{\mathrm{h}} 20^{\mathrm{m}}$ ed alle $22^{\mathrm{h}} 15^{\mathrm{m}}$ rispettivamente.

Sempre a Santa Sofia, una scossa di IV grado si verificò alle $4^{\text {h }}$ del 20 aprile ed un'altra, pure di IV grado, alle $00^{\mathrm{h}} 50^{\mathrm{m}}$ del 21 aprile.

Una scossa di IV grado, infine, fu avvertita a Galeata il 27 aprile alle $22^{\mathrm{h}} 17^{\mathrm{m}}$. 
Il 30 aprile altri movimenti tellurici di mediocre intensità furono avvertiti a Santa Sofia, a S. Piero in Bagno e a Galeata. Ma essi, come si vedrà al numero seguente, furono originati da un ipocentro situato alquanto più a sud di quello da cui irradiarono i sismi di cui si è parlato.

85. 30 aprile

Pieve S. Stefano (Arezzo)

Grado VI

Era appena terminato il periodo simico della zona di Santa Sofia, quando, una trentina di chilometri più a sud, s'iniziò un'altra serie di movimenti tellurici.

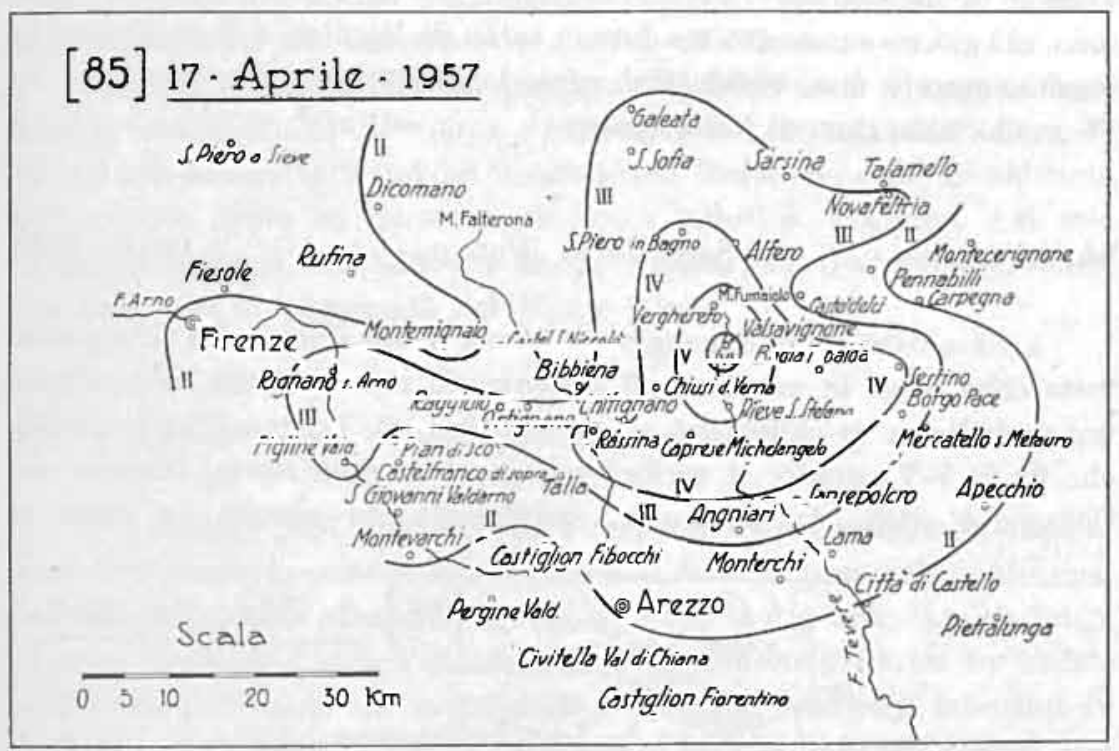

Fig. 54

Alle $7^{\mathrm{h}} 05^{\mathrm{m}}$ circa del 30 aprile 1957 , una forte scossa sussultoria si verifico nel territorio del comune di Pieve S. Stefano colpendo in particolar modo la frazione di Valsavignone ove si ebbero leggiere fenditure in diverse abitazioni. Solo in quest'ultima località, situata circa 8 chilometri a sud del Monte Fumaiolo, fu riscontrata la maggiore intensità della scossa (VI grado); è da ritenere pertanto che nei suoi pressi immediati debba essere stato l'epicentro, alle cui coordinate, data l'estrema limitatezza della zona megasismica, possono con buona approssimazione essere assegnati i valori $43^{\circ} 43^{\prime} \mathrm{N}$ e $12^{\circ} 02^{\prime} \mathrm{E}$. 
La scossa fu forte a Pieve $S$. Stefano e a Verghereto; sensibile a $S$. Piero in Bagno, Sestino, Caprese Michelangelo, Rassina (fraz. di Castel Focognano) e Chiusi della Verna; leggera a Galeata, Santa Sofia, Sarsina, Novafeltria, Borgo Pace, Sansepolero, Anghiari, Ortignano Reggiolo, Rignano sull'Arno e Figline Valdarno. Con leggerissima intensità fu percepita nelle città di Firenze e di Arezzo, in varie localltà delle rispettive province ed in altre localltà delle province di Forli e di Pesaro. La figura 54 ne dà l'approssimativa estensione macrosismica.

L'ora della scossa all'epicentro dedotta dalle registrazioni ottenute negli Osservatorî di Firenze, di Roma e di Prato, può ritenersi $07^{\mathrm{h}} 05^{\mathrm{m}} 08^{\mathrm{h}}$.

Nella stessa giornata si ebbero delle repliche di mediocre intensità verso le $07^{\mathrm{h}} 10^{\mathrm{m}}, 07^{\mathrm{h}} 30^{\mathrm{m}}, 09^{\mathrm{h}} 32^{\mathrm{m}}$ e verso le $10^{\mathrm{h}} 05^{\mathrm{m}}$. Altre repliche seguirono nel giorno successivo ma furono tutte di leggiera o leggerissima in tensità, eccetto una, verificatasi verso le $02^{\mathrm{h}} 10^{\mathrm{m}}$ che fu avvertita di IV grado nella zona di Valsavignone.

86. 3 maggio

Sant'Ilario (Potenza)

Grado V-VI

A circa $04^{\mathrm{h}} 07^{\mathrm{m}}$ del 3 maggio 1957 , una scossa d'intensità abbastanza forte ebbe luogo in provincia di Potenza, in una ristretta zona situata una quindicina di chilometri a sud del Vulture. La massima intensità, che fu di V-VI grado, si verificò nel paese di Sant'Ilario, frazione del Comune di Atella. Ivi la scossa, ondulatoria, fu sentita da tutta la

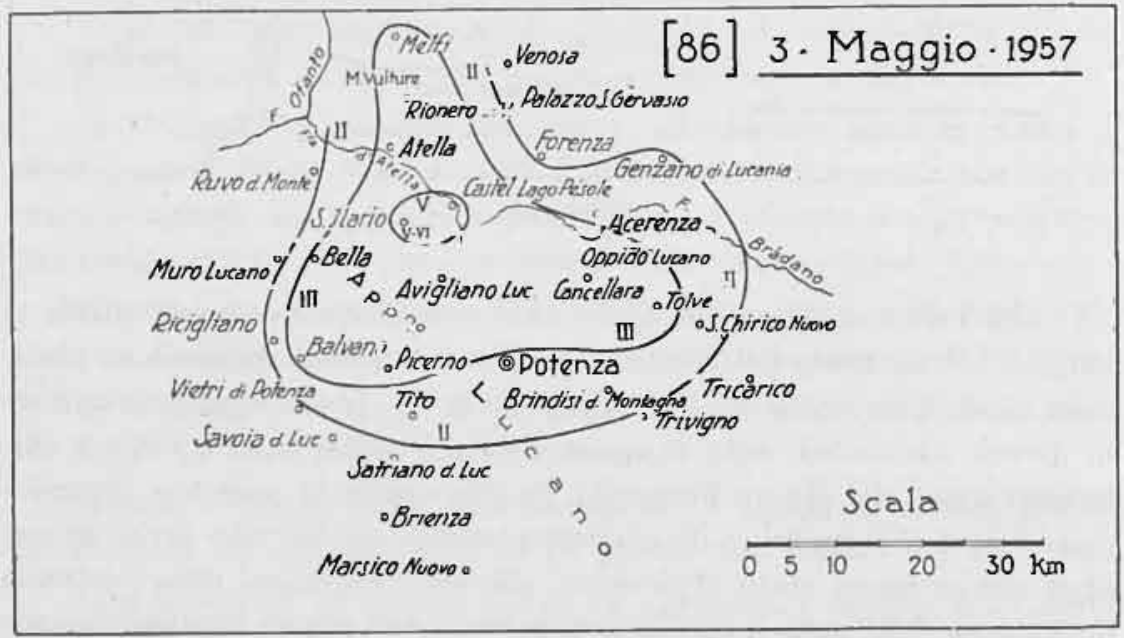

Fig. 55 
popolazione con grande spavento e causò leggerissime fenditure in alcune case. Nel vicino paesetto di Lagopesole, noto per il mirabile Castello svevo, il movimento tellurico fu sentito di $\mathrm{V}$ grado e con carattere pure ondulatorio.

In tutte le altre località vicine l'intensità fu alquanto minore sicchè è da ritenere che l'epicentro sia stato nei pressi immediati di Sant'Ilario. Con approssimazione, crediamo, sufficiente, possono essere assunti per le coordinate epicentrali i valori $40^{\circ} 48^{\prime} \mathrm{N}$ e $15^{\circ} 42^{\prime} \mathrm{E}$.

L'area macrosismica, come risulta dalla figura 55 , fu di piccola estensione. La scossa fu avvertita leggermente nei comuni di Melfi, Rionero in Vulture, Tolve, Cancellara, Picerno, Balvano e Bella, tutti della provincia di Potenza. Una piccola traccia di registrazione se ne ebbe dagli apparecchi dell'Osservatorio sismico di Messina.

Un'altra scossa si ebbe in provincia di Potenza il 3 maggio 1957. Essa si verificò alle $11^{\mathrm{h}} 55^{\mathrm{m}}$ circa e fu sentita con intensità di IV-V grado nel comune di Lauria, a sud del Monte Sirino. Searsissime notizie abbiamo peró potuto avere su questo movimento tellurico. Possiamo dire solo ch'esso si propagò sensibilmente fino a Maratea, sul Golfo di Policastro, e a Latronico in prossimità del Monte Alpi.

87. 6 maggio

Naso (Messina)

Grado V

Il 6 maggio 1957 , alle $04^{\mathrm{h}} 14^{\mathrm{m}}$ circa, una scossa sussultoria-ondulatoria di V grado si verificò nella cittadina di Naso, in provincia di Messina, propagandosi, come mostra la fig. 56, in modo molto irregolare sia lungo la costa tirrenica che nell'entroterra. Il movimento tellurico fu sentito però fortemente solo nel comune di Naso; con mediocre intensità fu avvertito a S. Agata Militello, Militello Rosmarino, Longi, Galati Mamertino, Capo d'Orlando, Piraino, S. Angelo di Brolo, Fùrnari e, isolatamente, nel Comune di Roccella Valdemone. Fu registrato negli Osservatorî di Messina e di Reggio Calabria con inizi, rispettivamente, alle $04^{\mathrm{h}} 14^{\mathrm{m}} 01^{\mathrm{s}}, 3$ e $04^{\mathrm{h}} 14^{\mathrm{m}} 02^{\mathrm{s}}, 8$.

Nella stessa giornata, a brevi distanze di tempo l'una dall'altra, seguirono nella zona di Naso altre scosse tutte di leggera intensità, l'ultima delle quali ebbe luogo alle $06^{\text {h }} 40^{\mathrm{m}}$ circa. Altre scosse, pure leggiere, avvertite anche a Brolo e a S. Agata Militello, si ebbero il $1^{\circ}$ giugno 1957.

Alquanto dubbia, data l'irregolare distribuzione delle intensità, appare l'ubicazione dell'epicentro. Forse le scosse predette rappresentano 
un lieve risveglio di qualcuno dei centri sismici esistenti lungo quel tratto della costa sicula che si estende da Capo d'Orlando a Capo Calava, centri ben noti per le intense manifestazioni telluriche da essi irradiate nei

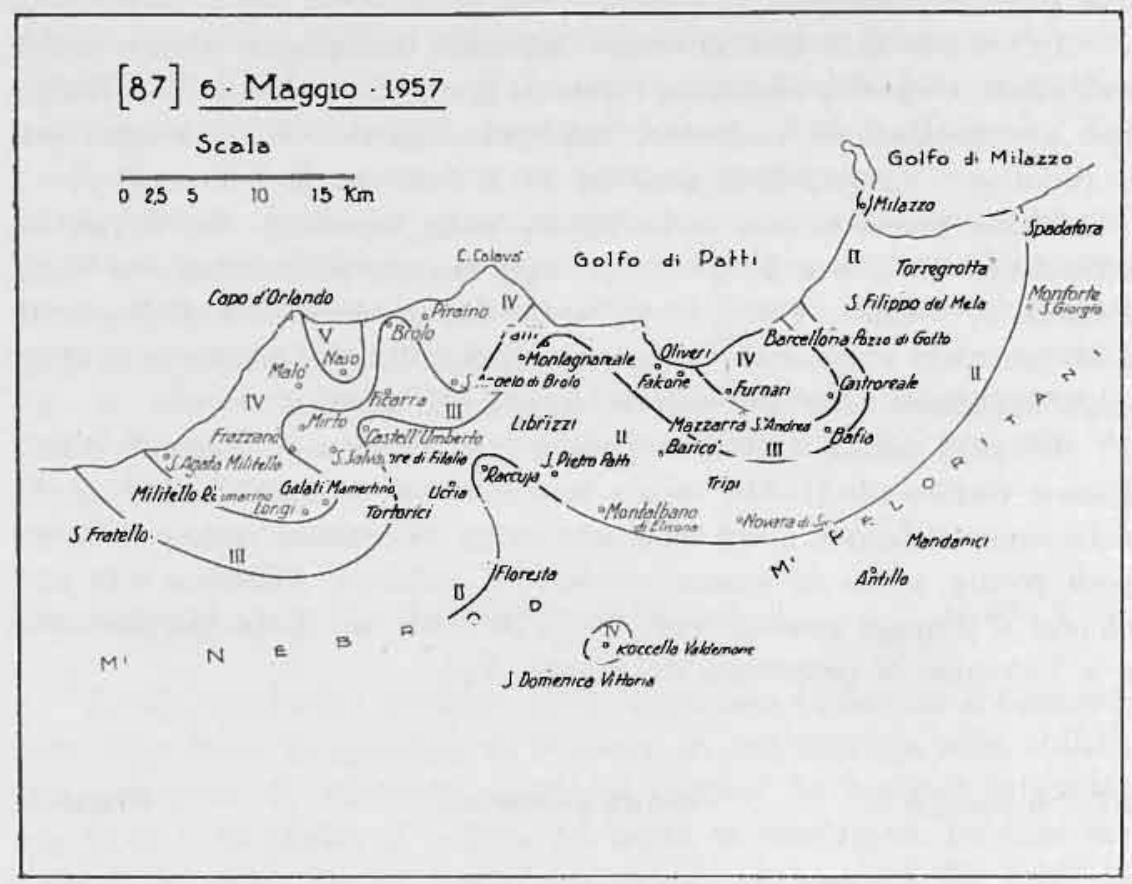

Fig. 56

secoli passati e che anche in tempi recenti (come, per esempio, il 15 dicembre 1931) hanno dato segni di attività con scuotimenti d'intensità all'incirca uguale a quella del sisma del 6 gennaio 1957, di cui ora si è detto.

\section{11 maggio \\ Sambuca di Sicilia (Agrigento) \\ S. Margherita Belice ( " )}

Grado V

Pochi giorni dopo i movimenti tellurici della zona di Naso, due altre scosse si verificarono in Sicilia, questa volta però nella parte sud-occidentale dell'Isola, e precisamente in provincia di Agrigento nella zona compresa fra le valli dei fiumi Belice e Verdura.

La prima scossa, che fu registrata debolmente nell'Osservatorio di Messina, avvenne alle $01^{\mathrm{h}} 45^{\mathrm{m}}$ eirca dell'11 maggio e fu sentita con in- 
tensità di V grado a Sambuca di Sicilia e a S. Margherita di Belice. Ebbe carattere sussultorio, una durata di 3-4 secondi e fu accompagnata da un forte boato. A Caltabellotta fu avvertita sensibilmente (IV grado) e con carattere pure sussultorio. D'intensità tra leggiera e mediocre (IIIIV grado) risultò nella città di Sciacca, nei cui pressi, com'è noto, esiste un centro sismico, probabilmente sottomarino, di discreta attività.

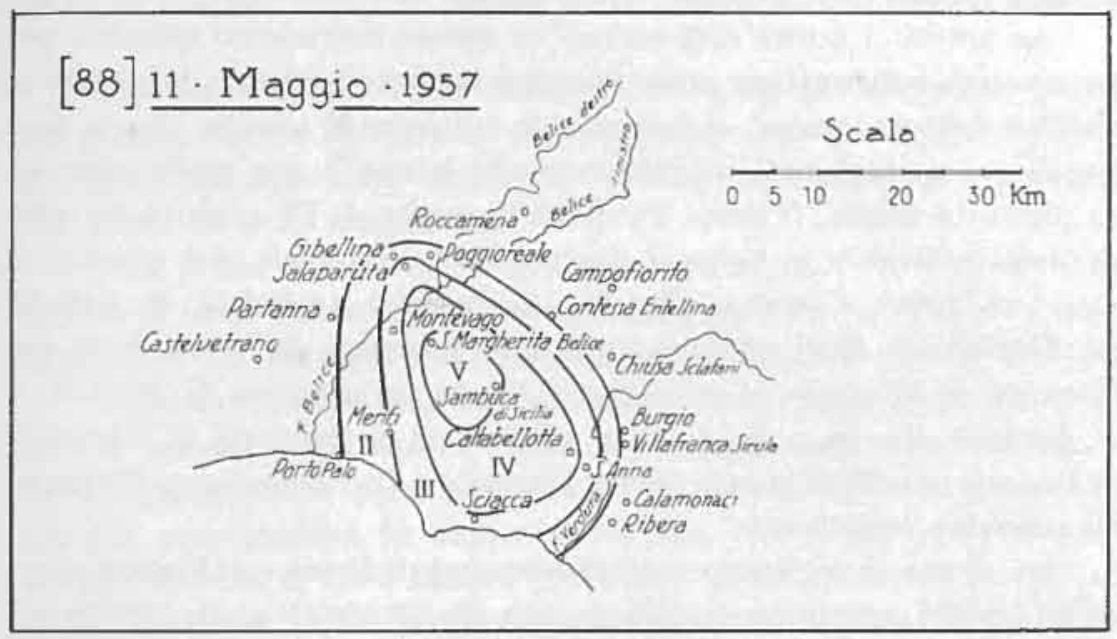

Fig. 57

La propagazione macrosismica, come appare dalla fig. $5 \bar{\tau}$, fu assai limitata. La scossa venne infatti percepita con intensità leggiera o leggerissima a $S$. Anna (fraz. di Caltabellotta) e nei comuni di Menfi, Montevago, Gibellina, Salaparuta, mentre passò del tutto inosservata nei centri di Ribera, Villafranca Sicula, Burgio, Chiusa Sclafani, Contessa Entellina, Partanna.

La seconda scossa, non registrata in alcun Osservatorio, si verificò appena un'ora dopo la prima, alle $02^{\mathrm{h}} 43^{\mathrm{m}}$ circa. Essa risultò però sensibile solo a Sciacca, ove raggiunse un'intensità di IV-V grado provocando vivo allarme nella popolazione. Fra le località sopra menzionate, solo a Sambuca di Sicilia fu leggermente avvertita; nelle altre, o passò del tutto inosservata o fu percepita solo da qualche persona e con lievissima intensità.

Eे da ritenere pertanto che le due scosse siano state originate da centri sismici diversi. La seconda, probabilmente, da quello di Sciacca, sopra accennato, e la prima da un centro sismico situato fra Sciacca e Sambuca di Sicilia. 
Il 16 maggio 1957 , alle $22^{\mathrm{h}} 52^{\mathrm{m}}$ circa, una scossa sismica di $\mathrm{V}$ grado colpi il territorio del comune di Force, propagandosi di là con intensità via via decrescente a quasi tutta la provincia di Ascoli Piceno ed anche a molte località delle province di Macerata e di Teramo.

Le notizie a nostra disposizione su questo movimento tellurico, per quanto non sufficienti per poter tracciare le curve isosiste e delimitare la totalità dell'area scossa, ci permettono tuttavia di asserire che la propagazione macrosismica, in relazione alla intensità non molto elevata, fu piuttosto ampia. Il sisma fu infatti avvertito di IV grado nella città di Ascoli Piceno e in tutto il suo territorio comunale, nei comuni di Castel di Lama, Castorano, Rotella, Montelparo, Amandola, S. Vittoria in Matemano e Monterubbiano, tutti della provincia di Ascoli. Pure con intensità di IV grado fu avvertito a Nereto, in provincia di Teramo; a S. Severino Marche, a Mogliano e a Tolentino, in provincia di Macerata. A Rapagnano e Montegiorgio (Ascoli Piceno) e a Torricella Sicura (Teramo) fu percepito leggermente.

La scossa fu registrata negli Osservatorî di Roma e di Firenze, ove i primi impulsi arrivarono rispettivamente alle $22^{\mathrm{h}} 53^{\mathrm{m}} 34^{\mathrm{s}}$ e alle $22^{\mathrm{h}} 53^{\mathrm{m}} 40^{\mathrm{s}}$.

Il 21 maggio 1957, dai sismografi di quasi tutti gli Osservatorí italiani e di molti Osservatori stranieri venne registrato un sisma di origine probabilmente profonda e per il cui epicentro, dall'U.S.C.G.S., vennero assegnate le coordinate $38^{\circ} 1 / 2 \mathrm{~N}$ e $14^{\circ} \mathrm{E}$, individuanti un punto situato nel Basso Tirreno, una trentina di chilometri ad ovest dell'Isola di Alicudi.

Il sisma fu macrosismicamente avvertito nella provincia di Palermo, ma assai scarse sono le notizie che abbiamo potuto raccogliere al riguardo. Sappiamo solo che il movimento tellurico risultò sensibile nella città di Palermo, ove destò un certo allarme nella popolazione, nelle località costiere del Golfo di Carini e nel territorio del comune di Torretta, mentre passò inosservato a Bagheria e ad Altavilla Milicia.

Il primo impulso, nelle registrazioni degli Osservatorî di Messina e Reggio Calabria, i più vicini alla zona epicentrale, si ebbe alle $12^{\mathrm{h}} 44^{\mathrm{m}} 30^{\mathrm{s}}, 4$ e alle $12^{\mathrm{h}} 44^{\mathrm{m}} 32^{\mathrm{s}}, 2$ rispettivamente. 
Una scossa, che può considerarsi premonitoria di quella di cui ora si è detto, fu avvertita a Palermo alle $20^{\mathrm{h}} 58^{\mathrm{m}}$ circa del 20 maggio. Essa ebbe lo stesso epicentro e fu registrata in numerosi Osservatori italiani e stranieri.

91. 22 maggio

Una breve ripresa di movimenti tellurici, che può considerarsi come un prolungamento deli'intenso periodo sismico svoltosi durante il mese di aprile neli'Appennino Emiliano, si ebbe nella zona di Santa Sofia nei giorni 21 e 22 maggio 1957 .

Una prima scossa, di IV grado, accompagnata da rombo e della durata di circa 4 secondi, fu avvertita a Santa Sofia alle $12^{\mathrm{h}} 10^{\mathrm{m}}$ circa del 21 maggio. Ad essa segui una replica di leggera intensità alle $12^{\mathrm{t}} 30^{\mathrm{m}}$ circa.

Il giorno 22 , verso le $12^{\mathrm{h}} 05^{\mathrm{m}}$, una scossa forte (V grado) e della durata di 3-4 secondi colpi ancora Santa Sofia aggravando sensibilmente le lesioni già prodotte nelle abitazioni dalle scosse del mese precedente. La sua propagazione fu alquanto limitata: venne avvertita di IV-V grado a Galeata, di III a Rocca S. Casciano e a Verghereto, di II a Premilcuore. Passò del tutto inavvertita nei comuni di Sarsina, Forlimpopoli, S. Arcangelo di Romagna e Cesena.

Di questa scossa si ebbero registrazioni negll Osservatorí di Firenze, di Prato e di Bologna.

L'ultima replica, avvertita a Santa Sofia con lieve intensità, si verificò alle $15^{\text {th}} 30^{\text {m }}$ circa del 22 maggio.

\section{22 maggio}

II 22 maggio 1957 , alle $12^{\text {h }} 4^{\mathrm{m}}$ circa, una scossa teliurica fu avvertita, con intensità quasi ovunque modesta, in diverse località dell'Appennino Calabro compreso fra il Massiccio dell'Aspromonte e la cosiddetta Gola di Marcellinara, l'insellatura che mette in comunicazione gli opposti Golfi di Squillace e di S. Eufemia. In una piccolissima zona peraltro, comprendente il solo comune di Mammola (Reggio Calabria), la scossa risultò molto forte raggiungendo un'intensità che, dagli effetti dinamici denunciati, può essere valutata di VI grado almeno. Nel comune predetto essa fu infatti sentita da tutta la popolazione con spavento generale e fuga dalle case e determinò fenditure più o meno sensibill in 
numerosi fabbricati. La zona megasismica fu però, come si è detto, estremamente ristretta: località assai prossime a Mammola, come Grotteria e S. Giovanni di Gerace, avvertirono il movimento tellurico solo con mediocre intensità. L'epicentro può ritenersi pertanto ubicato proprio nei pressi di Mammola, in un punto alle cui coordinate geografiche possono con buona approssimazione essere assegnati i valori $38^{\circ} 22^{\prime} \mathrm{N}$ e $16^{\circ} 14^{\prime} \mathrm{E}$.

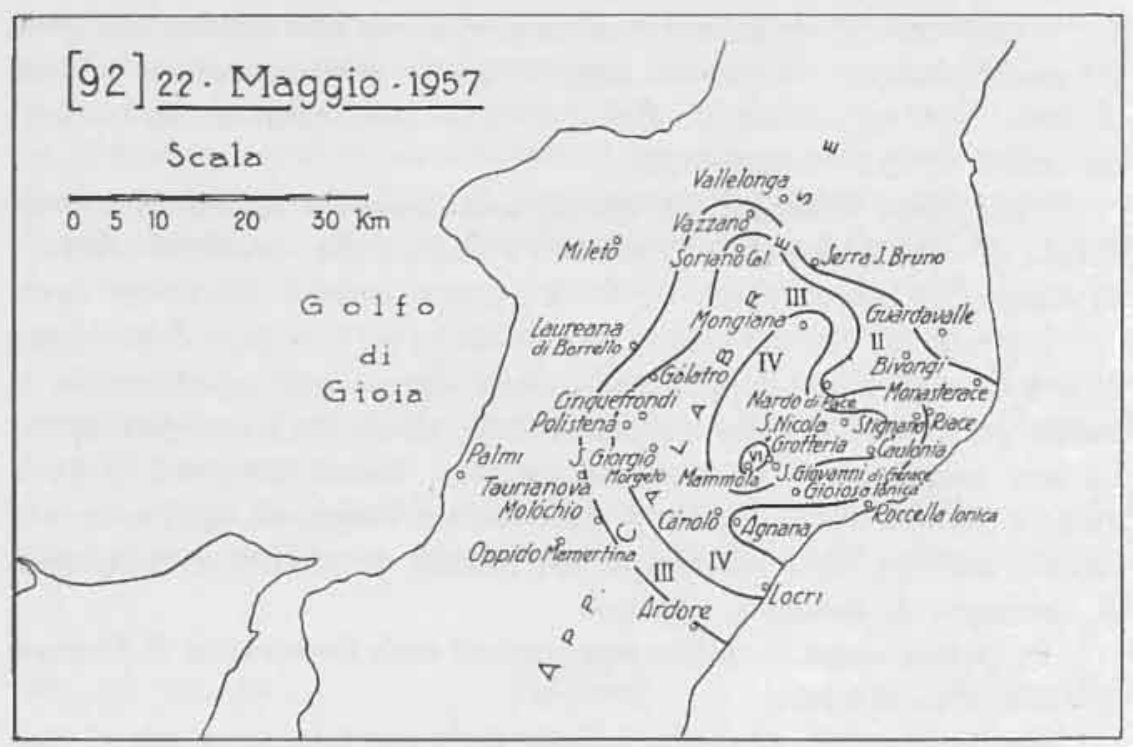

Fig. 58

La scossa fu avvertita mediocremente, oltre che nei comuni predetti, a Caulonia (Reggio Calabria) e a Mongiana (Catanzaro); leggermente a Locri, S. Giorgio Morgeto, Polistena, Cinquefrondi, Galatro, della provincia di Reggio, e a Soriano Calabro, in provincia di Catanzaro.

Dalla esiguità della zona ove la scossa risultò percettibile alle persone (fig. 58) si puó inferire che assai piccola dovette essere la profondità ipocentrale.

La scossa fu registrata solo negli Osservatorî di Reggio Calabria e di Messina: nel primo, con inizio alle $12^{\mathrm{h}} 58^{\mathrm{m}} 08^{\mathrm{s}}, 3$ e, nel secondo, alle $12^{\mathrm{h}} 53^{\mathrm{m}} 09^{\mathrm{s}}$. 
93. 29 maggio

Mammola (Reggio Calabria)

Grado V

Replica della scossa descritta nel numero precedente. Ebbe anche essa una propagazione limitatissima. Fu avvertita fortemente nel solo comune di Mammola e mediocremente nei comuni di S. Giovanni di Gerace e di Stignano. Leggera risultò a Mongiana, Grotteria e Locri; leggerissima a Nardodipace.

Non venne registrata in alcun Osservatorio.

94. 26 giugno

Force (Ascoli Piceno)

Grado V

Il 26 giugno 1957 si ebbero in provincia di Ascoli Piceno aleune scosse telluriche che possono considerarsi delle repliche di quella verificatesi il giorno 16 del mese precedente (n. 89).

La più forte. preceduta di pochi minuti da leggiere scossette, ebbe luogo alle $03^{\mathrm{h}} 25^{\mathrm{m}}$ circa e, come quella del 16 maggio, raggiunse la sua maggiore intensità (V grado) nel territorio del comune di Force, ove fu sentita con vivo allarme da tutta la popolazione. Sensibilmente fu avvertita a Montelparo, a S. Vittoria in Matemano, a Rotella e nella città di Ascoli Piceno; leggermente a Rapagnano, Montegiorgio, Amandola, Montefortino, Folignano e Montalto Marche.

Dalle scarse notizie relative alle località ove la scossa fu percepita con leggerissima intensità o passò completamente inosservata, sembra potersi dedurre che la sua propagazione dovette essere un po' inferiore a quella della scossa del 16 maggio.

Due repliche di minore intensità si ebbero durante la stessa giornata del 26 giugno. L'una, mediocre, avvenuta alle $05^{\mathrm{h}} 15^{\mathrm{m}}$ circa, fu avvertita a Force, a Montelparo e a Rotella; l'altra di IV-V grado, si verifico verso le $09^{\mathrm{h}} 38^{\mathrm{m}}$ e fu avvertita, oltre che nelle località ora citate, anche a Montegiorgio.

Tutte le scosse predette, nei comuni di Force e Montelparo, furono precedute da un rombo piuttosto intenso.

95. 19 luglio Spoleto (Perugia)

Grado VII

Nella seconda metà di luglio del 1957 un periodo sismico di breve durata ma piuttosto intenso ebbe luogo nel territorio spoletino. Le scosse ebbero tutte, anche la più forte, un'estensione macrosismica molto limi- 
tata e i danni da esse causati, numerosi e di una certa gravità, si verificarono specialmente nella città di Spoleto.

Il periodo sismico ebbe inizio alle $06^{\mathrm{h}} 54^{\mathrm{m}}$ circa del 17 luglio con una scossa preceduta da forti boati, avvertita solo a Spoleto e con mediocre intensità. Un'altra scossa, questa però molto forte ed avvertita anche nelle località limitrofe, avveniva circa mezz'ora dopo, alle $07^{\mathrm{h}} 25^{\mathrm{m}}$ circa. Essa fu nettamente sussultoria ed ebbe una durata di 4-5 secondi. A Spoleto causò la caduta di qualche comignolo e lievi fenditure in alcune vecchie case di Via Aurelio Saffi e del quartiere Monterone, che è situato nella parte della città più prossima alle pendici di Monte Luco. La popolazione, allarmata, si riversava all'aperto sostando a lungo nelle piazze e nei giardini, lontano dagli edifici. Macrosismicamente questa scossa ebbe una propagazione limitatissima, essendo stata avvertita fortemente solo a Spoleto e lievemente nelle località vicine. Microsismicamente si propagò fino a Roma, ove dai sismografi venne debolmente registrata con inizio alle $07^{\mathrm{h}} 29^{\mathrm{m}} 18^{\mathrm{s}}, 6$.

Seguirono altre scosse a breve distanza di tempo l'una dall'altra, tutte accompagnate da rombi. Una di forte intensità (V grado) avvenne alle $7^{\mathrm{h}} 35^{\mathrm{m}}$ ed altre due, leggiere, intorno alle $8^{\mathrm{h}} 40^{\mathrm{m}}$ del giorno 17 .

Sembrava che con queste ultime scosse i movimenti tellurici fossero completamente cessati ed ogni timore era oramai scomparso nella popolazione, quando la mattina del 19 luglio, alle $10^{\mathrm{h}} 04^{\mathrm{m}}$ circa, sopravveniva un'altra fortissima scossa, la più violenta di tutto il periodo sismico, spargendo nuovamente il più vivo panico fra gli abitanti di Spoleto ed arrecando sensibili danni agli edifici della città. La scossa spiegò la sua maggiore intensità, corrispondente al VII grado della scala Mercalli, in quella parte di Spoleto che si estende alla sinistra del Torrente Tessino. $\mathrm{Fu}$ in tale zona appunto che numerosi edifici, pubblici e privati, ebbero a subire notevoli danni, consistenti in crolli di comignoli, lesioni di varia entità nei muri divisori ed in quelli portanti, distacchi verticali nelle connessioni dei muri e distacchi orizzontali in corrispondenza di soffitti e di tetti. Particolarmente colpite risultarono le abitazioni dei quartieri Monterone e S. Paolo. In quest'ultimo, anzi, alcune case dovettero, per precauzione, essere sgomberate e puntellate. Gravemente danneggiata fu la storica Porta Loreto e lievi lesioni si verificarono in alcuni punti delle antiche mura urbane. Qualche danno fu riscontrato anche nella parte occidentale della Rocea Albornoziana, imponente costruzione del XIVo secolo eretta sul Colle $\mathrm{S}$. Elia, in posizione dominante sulla città.

Pochi e di molto minor rilievo furono i danni nella zona di Spoleto situata alla destra del Torrente Tessino. Ivi la scossa si manifestò con una 
intensità valutabile di VI grado. Anche sul Monte Luco, la pittoresca montagna che col suo verde manto di lecei si eleva immediatamente a sud-est della città, da cui la separa la stretta valle del Tessino, la scossa fu avvertita molto fortemente ma non arreed sensibili danni. Con fortissima intensità venne invece colpita la frazione di Collerisana, situata un paio di chilometri ad ovest di Spoleto, e assai elevata fu ivi la percentuale delle case più o meno gravemente danneggiate.

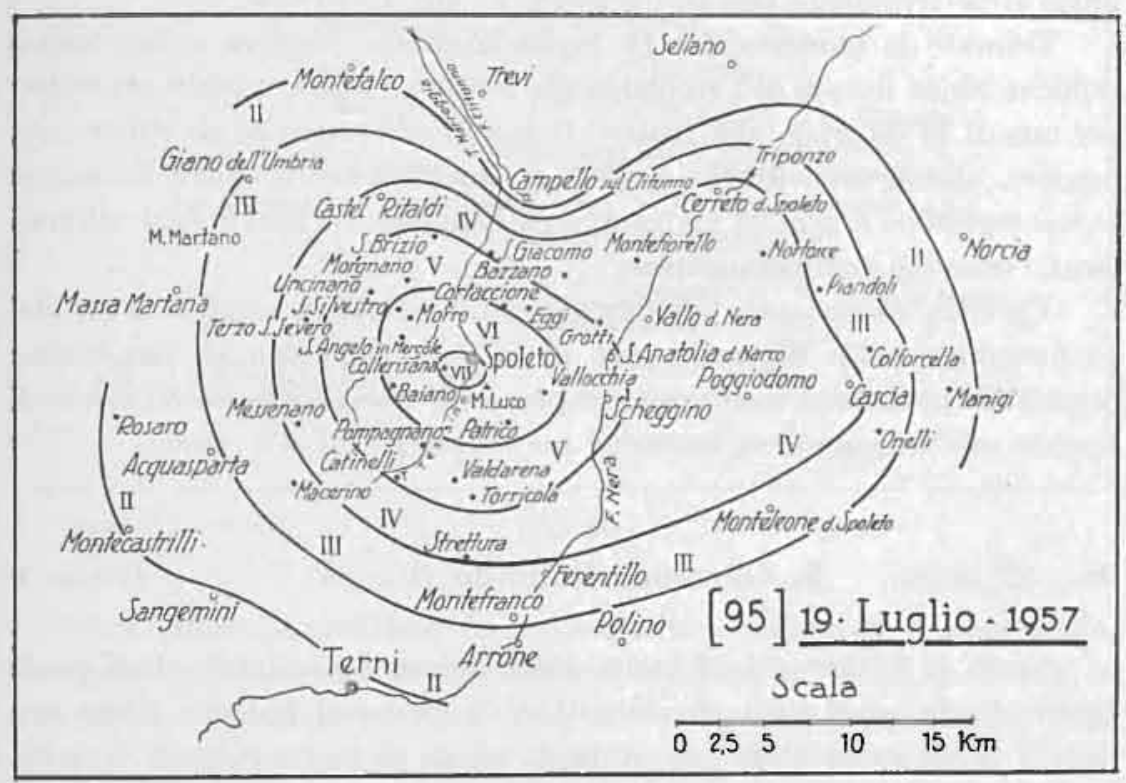

Fig. 59

Poichè in nessun'altra località la scossa raggiunse un'intensità corrispondente al VII grado, è da ritenere che l'epicentro si sia trovato fra la zona sud occidentale di Spoleto e la frazione di Collerisana, in un punto cui, con buona approssimazione, possono essere assegnate le coordinate $44^{\circ} 44^{\prime} \mathrm{N}$ e $12^{\circ} 43^{\prime} 30^{\prime \prime} \mathrm{E}$.

Certamente assai piccola fu la profondità dell'ipocentro. La scossa infatti, in relazione alla notevole sua intensità nella ristretta zona megasismica, ebbe una propagazione molto limitata. Si manifestò con intensità molto forte, arrecando qualche danno in abitazioni vecchie e di difettosa costruzione, nelle frazioni di Cortaccione, S. Silvestro, S. Angelo in Mercole, Baiano, Patrico e Vallocchia. Di V grado risultò nelle frazioni di Uncinano, S. Brizio, Eggi, Catinelli, Pompagnano, Valdarena, 
Torrecola e nei comuni di Scheggino e S. Anatolia di Narco, in Val di Nera. Di IV grado fu avvertito a Bazzano, Messenano, S. Giacomo e Strettura (frazioni di Spoleto) e nei comuni di Castel Ritaldi, Cerreto di Spoleto, Vallo di Nera, Poggiodomo e Cascia.

La fig. 59 indica l'estensione approssimativa della zona ove la scossa si rese percettibile all'uomo. Con intensità strumentale il moto sismico giunse fino a Roma e Firenze, ove fu registrato dagli apparecchi con inizio rispettivamente alle $10^{\mathrm{h}} 04^{\mathrm{m} 2} 2 \tau^{\mathrm{s}}, 8$ e alle $10^{\mathrm{h}} 04^{\mathrm{m}} 34^{\mathrm{s}}$.

Durante la giornata del 19 luglio si ebbero leggiere e brevissime repliche (della durata di 1 secondo) alle $10^{\mathrm{h}} 30^{\mathrm{m}}, 11^{\mathrm{l}} 20^{\mathrm{m}}, 12^{\mathrm{l}} 02^{\mathrm{m}}$ e $14^{\mathrm{h}} 05^{\mathrm{m}}$; poi una di IV-V grado alle $16^{\mathrm{n}} 15^{\mathrm{m}}$. Il giorno successivo se ne ebbero tre, leggiere, alle $00^{\mathrm{h}} 30^{\mathrm{m}}, 01^{\mathrm{h}} 45^{\mathrm{m}}$ e $06^{\mathrm{h}} 45_{\mathrm{ur}}$ rispettivamente. Altre lievissime scosse seguirono fino al 26 luglio, dopo il qual giorno i movimenti tellurici locali cessarono completamente.

Un'altra scossa assai lieve fu avvertita a Spoleto verso le $5^{\text {li }} 40^{\mathrm{m}}$ del 12 novembre 1957 . Si trattò però di un fenomeno sismico esocentrico originatosi nella zona montuosa compresa fra Norcia, Cascia e Cerreto di Spoleto ove raggiunse un'intensità non superiore al IV grado.

96. 27 luglio

S. Giovanni Rotondo (Foggia)

Grudo V

Verso le $13^{\mathrm{h}} 58^{\mathrm{m}}$ del 27 luglio 1957 , una scossa valutata di $\mathrm{V}$ grado fu sentita da quasi tutti gli abitanti di S. Giovanni Rotondo. Ebbe una durata di 2-3 secondi e fu preceduta da rombi piuttosto intensi. Si trattò però di un fenomeno di carattere locale poichè al di là di una decina di chilometri da S. Giovanni Rotondo il movimento tellurico passò del tutto inavvertito.

Qualche giorno prima, e precisamente alle $12^{\mathrm{h}} 09^{\mathrm{m}}$ del 22 luglio, una scossa di mediocre intensità (IV grado), anche questa di earattere locale, era stata avvertita a Monte S. Angelo.

Nessun Osservatorio registrò questi movimenti tellurici.

97. 23 agosto Sillano-Piazza al Serchio (Lucea) Grado V

La mattina del 23 agosto 1957 , alle $06^{\mathrm{h}} 05^{\mathrm{m}} 30^{\mathrm{s}}$, una sensibile scossa di terremoto fu sentita in alcune località della Garfagnana. Alle coordinate epicentrali, in base alle registrazioni ottenute negli Osservatori di Firenze, di Pavia e di Bologna, furono dall'I.N.G. assegnati i valori $44^{\circ} 12^{\prime} \mathrm{N}$ 
e $10^{\circ} 18^{\prime} \mathrm{E}$, i quali individuano un punto situato nell'alta valle del Serchio, fra Sillano e Piazza al Serchio. In questi due paesi la scossa, valutata di V grado, fu sentita da tutta la popolazione, con forte tremolio di oggetti e con risveglio delle persone ancora addormentate.

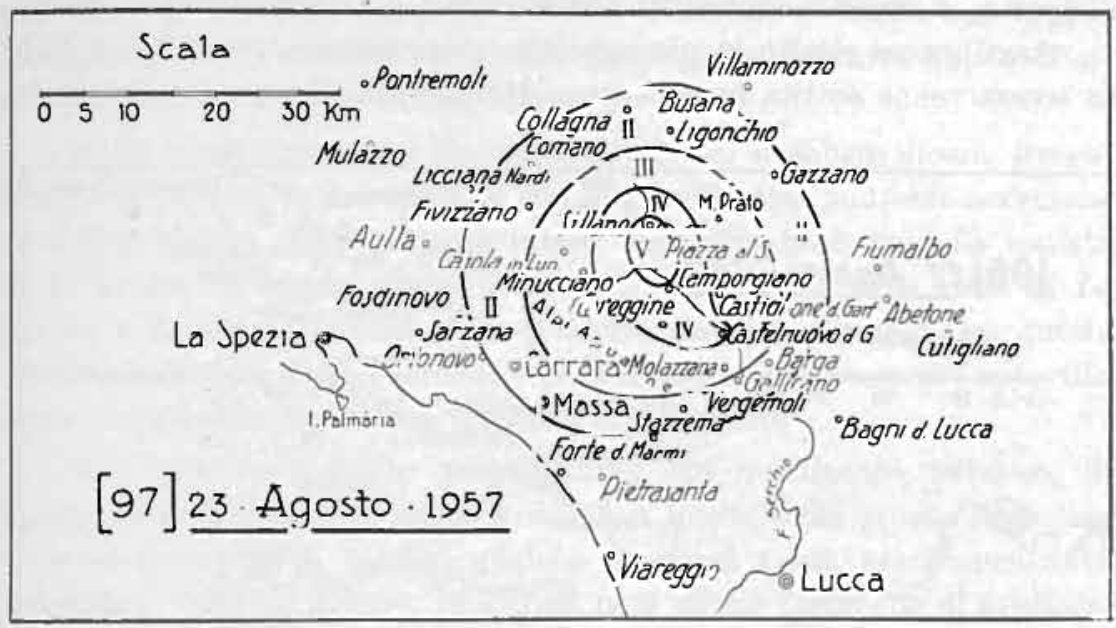

Fig. 60

Con intensità mediocre fu avvertita nei comuni di Camporgiano, Carèggine e Castelnuovo di Garfagnana; leggiera, in quelli di Casola in Lunigiana, Molazzana e Castiglione di Garfagnana; leggerissima, nelle città di Massa e Carrara ed in varie località delle province di MassaCarrara e di Reggio Emilia.

In figura 60 si hanno le isosiste del movimento tellurico.

Non si ebbe notizia di alcuna replica.

98. 27 agosto

Il 27 agosto 1957 ebbe luogo nell'Appennino Modenese una notevole scossa di terremoto, la quale, per la sua vasta propagazione microsismica, potè essere registrata in tutti gli Osservatorî italiani ed anche in molti Osservatori esteri. L'ora origine, la magnitudo e le coordinate epicentrali del sisma vennero calcolate dall'I.N.G. in base alle registrazioni ottenute negli Osservatorî italiani e si ebbero per esse rispettivamente i valori seguenti:

$$
\mathrm{H}=12^{\mathrm{h}} 54^{\mathrm{m}} 40^{\mathrm{s}} ; \quad \mathrm{I}=4,8 ; \quad \varphi=44^{\circ} 18^{\prime} \mathrm{N}, \quad \lambda=10^{\circ} 57^{\prime} \mathrm{E} .
$$


L'epicentro fu pertanto in provincia di Modena, sei chilometri circa a sud ovest di Zocca. In questa cittadina e nella zona circostante, la scossa, durata 3-4 secondi, ebbe carattere sussultorio e raggiunse un'intensità di VI grado. Fu sentita da tutti con spavento, sia nelle case che all'aperto, e causò fenditure in alcuni edifici di costruzione difettosa.

Assai estesa risultò la propagazione macrosismica (vedi figura 61). La scossa venne sentita fortemente a Montese e in diverse località della

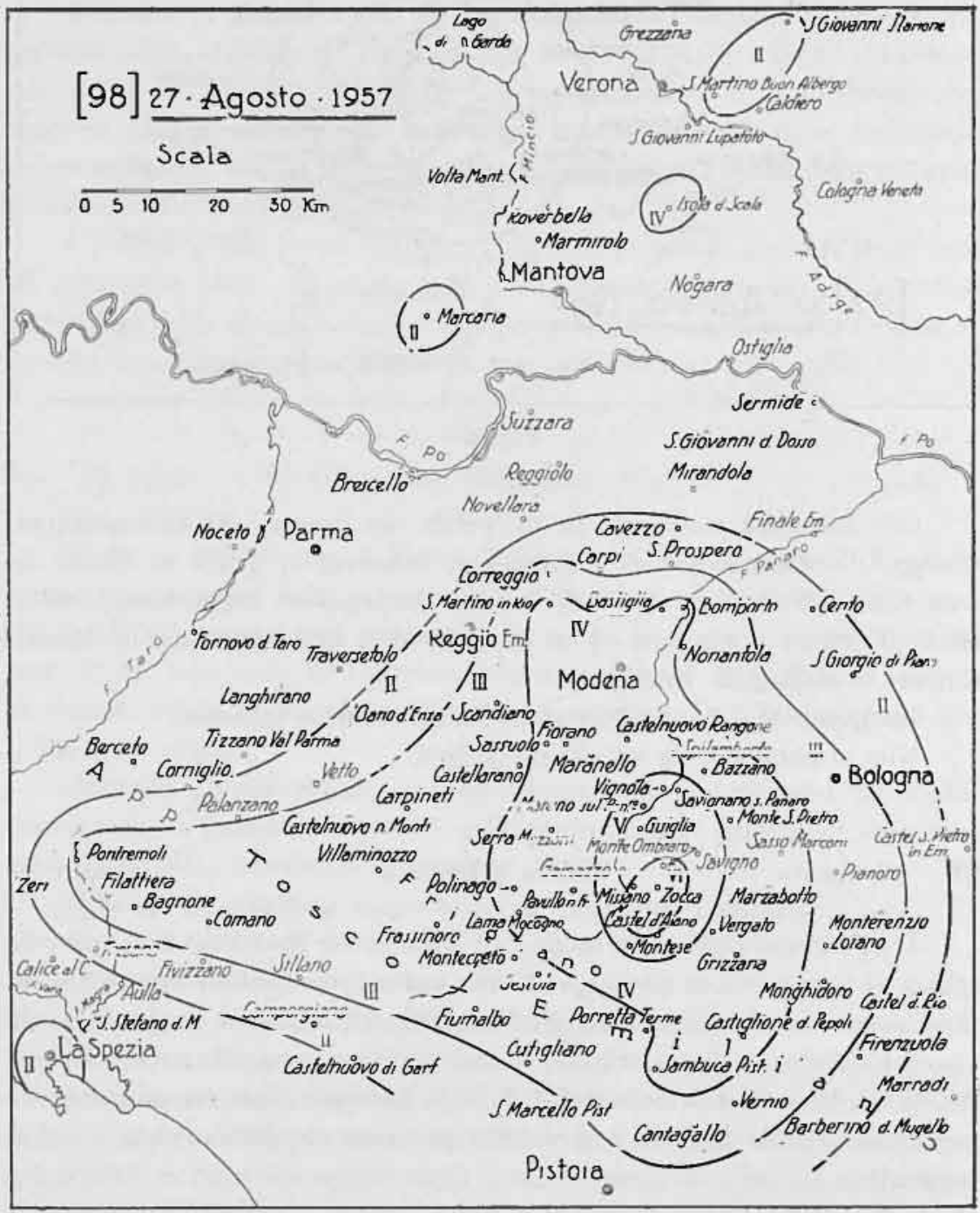

Fig. 11 
Valle del Panaro, come Vignola, Savignano sul Panaro e Marano sul Panaro; sensibilmente (IV grado) nella città di Modena e su tutta una ampia zona avente ai suoi limiti Bastiglia, Bomporto, Polinago e Sassuolo, della provincia di Modena, Sasso Marconi, e Castiglione de' Pepoli, della provincia di Bologna, Sambuca Pistoiese (Pistoia) e S. Martino in Rio (Reggio Emilia). Verso ovest giunse con leggera intensità fino a Pontremoli, nella Valle del Fiume Magra.

Nella propagazione del movimento tellurico si ebbero alcune irregolarità che possono essere spiegate con una profondità piuttosto accentuata dell'ipocentro. Esternamente infatti alla zona limitata della isosista di II grado, la scossa venne isolatamente avvertita nella città di $L a$ Spezia e in alcune localltà della Pianura Padana. Citiamo, fra queste ultime, Isola della Scala (Verona), ove la scossa fu distintamente avvertita come ondulatoria N-S e con intensità di IV grado.

Non si ebbero scosse premonitorie. Un movimento tellurico, di epicentro probabilmente assai prossimo a quello della scossa precedentemente descritta, si verificò qualehe mese più tardi, precisamente il 6 novembre verso le $01^{\mathrm{h}} 05^{\mathrm{m}}$. Si trattò però di un fenomeno di mediocre intensità e di propagazione assai limitata, avvertito di IV-V grado a Montese, di IV a Zocca e più leggermente nelle circostanti località.

\section{18 ottobre Satriano di Lucania (Potenza) Grado V}

Una scossa di terremoto abbastanza forte si verificò nell'Appennino Lucano a $07^{\mathrm{l}} 14^{\mathrm{m}}$ eirca del 18 ottobre 1957 , raggiungendo la sua maggiore intensità (V grado) a Satriano di Lucania in provincia di Potenza. In detta località la scossa, che fu preceduta e accompagnata da rombi, risultò ondulatoria ed ebbe una durata di 6-7 secondi.

Assai scarse sono le notizie pervenuteci su questo movimento tellurico. Sappiamo solo che esso venne avvertito con intensità di IV grado nei comuni di Brienza e di Balvano e che la sua propagazione risultò piuttosto limitata essendo passato del tutto inosservato in paesi situati intorno a Satriano di Lucania entro un raggio non superiore ad una ventina di chilometri.

La scossa fu registrata nell'Osservatorio di MIessina e, molto debolmente, anche in quelli di Reggio, di Taranto e di Roma.

Una lleve repllca si ebbe, nella stessa giornata, alle $14^{\mathrm{h}}$ circa. 
100. 26 ottobre Ramiseto (Reggio Emilia) Grado V-VI

Poco dopo la mezzanotte del 25 ottobre 1957, una scossa di terremoto, immediatamente seguita da un intenso boato, si abbattè

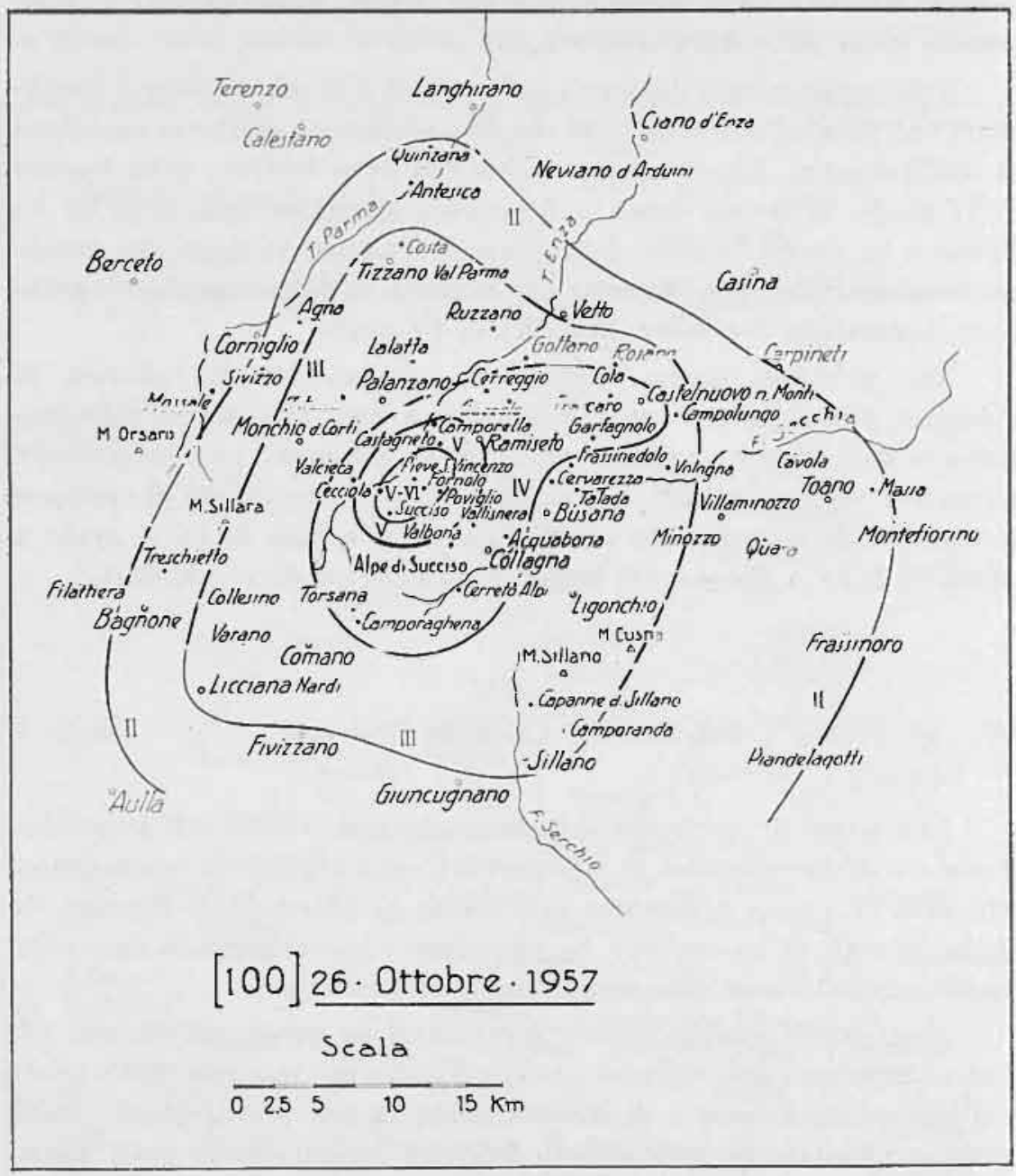

Fig. 62

all'improvviso su alcuni paesi dell'Appennino Reggiano compreso fra le alte valli del Fiume Enza e del Fiume Secchia. Il movimento tellurico ebbe una durata di 4-5 secondi e colpi specialmente le frazioni 
di Pieve S. Vincenzo, Fornolo, Poviglio, Cècciola e Succiso, tutti appartenenti al comune di Ramiseto (Reggio Emilia), causando in molte case la caduta di calcinacci, lievi danni nei tetti e fenditure di modesta entità nelle murature meno solide. Poichè però tall danni si verificarono in edifici vecchi e di costruzione molto difettosa, si può ritenere che la scossa, nelle predette località, non abbia superato l'intensità di V-VI grado. L'epicentro cadde molto probabilmente nella ristretta zona racchiudente i paesi sopra nominati e alle sue coordinate, data l'esiguità dell'area fortemente colpita, possono essere assegnati, con sufficiente approssimazione, i valori di $44^{\circ} 23^{\prime} \mathrm{N}$ e $10^{\circ} 12^{\prime} \mathrm{E}$.

La scossa non ebbe una propagazione macrosismica molto vasta. Essa venne sentita con intensità compresa fra il IV ed il V grado a Ramiseto (capoluogo) e nelle sue frazioni di Camporella e Castagneto; di IV grado fu avvertita in tutto il territorio comunale di Collagna, a Castelnuovo ne' Monti, in alcune sue frazioni (Costa de' Grassi, Garfagnolo, Frascaro) e in una parte del comune di Comano (frazioni di Torsana e Camporaghena); di leggera intensità risultò a Comano e nei comuni di Tizzano Val Parma, Monchio delle Corti, Licciana Nardi, Sillano, Ligonchio e Busana.

Dalla figura 62 si ha un'idea dell'estensione del movimento tellurico, il quale, oltre la provincia di Reggio Emilia, interessò macrosismicamente anche parti delle contigue province di Parma, Modena, Massa-Carrara e Luccic.

Sotto forma microsismica la scossa venne registrata nell'Osservatorio di Firenze con inizio alle $00^{\mathrm{h}} 02^{\mathrm{m}} 05^{\mathrm{s}}$ del 26 ottobre.

Nessuna scossa premonitoria, nè alcuna repllca, venne registrata 0 avvertita.

Numana (Aneona)
101. 11 novembre
Porto Recanati (Macerata) Grado VI

Il giorno 11 novembre 1957 , alle $22^{\mathrm{b}} 40^{\mathrm{m}}$ circa, si ebbe in provincia di Ancona un terremoto d'intensità molto forte il cui epicentro, a giudicare dall'andamento delle isosiste (v. fig. 63), fu molto probabilmente in mare, una decina di chilometri a sud-est del Monte Conero.

La zona megasismica, ove la scossa raggiunse un'intensità di VI grado con una durata di 7-8 secondi, si estese, sulla costa, da Numana a Porto Recanati e, neil'entroterra, fino a Castelfidardo. A Numana il movi- 
mento fu avvertito come sussultorio; a Porto Recanati e a Castelfidardo, prima come sussultorio e poi come ondulatorio. Contemporaneamente alla scossa in tutta la zona furono uditi rombi più o meno intensi.

La scossa fu sentita fortemente nella eittà di Ancona, a Camerano, a Potenza Picena e a Loreto; con mediocre intensità a Falconara Marit-

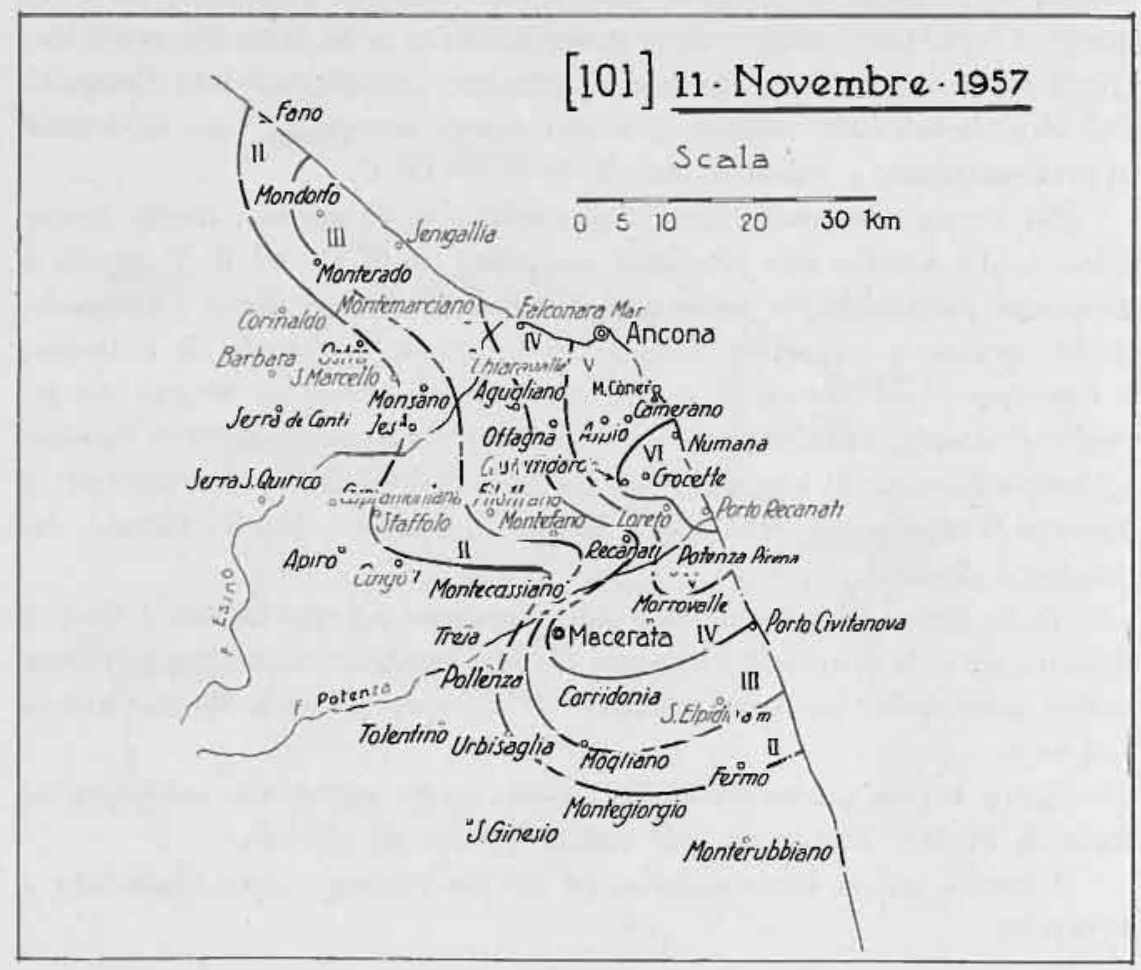

Fig. 63

tima, Offagna, Macerata e Morrovalle; leggermente a Mondolfo, Senigallia, Montemarciano, Chiaravalle, Agugliano, Filottrano, Mogliano e S. Elpidio a Mare. In tutte queste località la scossa fu avvertita come ondulatoria, eccetto a Loreto ove ebbe carattere prevalentemente sussultorio.

Come si rileva dalla figura 63, il movimento tellurico si propagò macrosismicamente lungo la costa in misura notevolmente maggiore che non verso l'entroterra. Eे questa una caratteristica comune a tutti i terremoti con epicentro nei pressi della zona costiera marchigiana. 
Il primo impulso delle onde sismiche, a Firenze ed a Roma, venne registrato rispettivamente alle $22^{\mathrm{h}} 40^{\mathrm{m}} 15^{\mathrm{s}}$ ed alle $22^{\mathrm{h}} 40^{\mathrm{m}} 16^{\mathrm{s}}$.

Seguirono tre repliche di minore intensità durante la prima metà del giorno 12. La prima, leggera, verso le $01^{\mathrm{h}} 40^{\mathrm{m}}$; la seconda, verificatasi alle $02^{\mathrm{h}} 44^{\mathrm{m}}$ circa, risultò di IV-V grado a Castelfidardo e a Porto Recanati, di IV a Numana, Loreto e Camerano, di III ad Ancona, Offagna, Chiaravalle e S. Elpidio a Mare; la terza avvenne alle $10^{\mathrm{h}} 40^{\mathrm{m}}$ circa e fu avvertita di IV-V grado a Castelfidardo e Porto Recanati, di IV a Camerano e Macerata, di III ad Offagna, Agugliano e S. Elpidio a Mare.

Un'altra scossa, avvertita ad Ancona con intensità di IV-V grado e più leggermente nelle località circostanti, si ebbe alle $03^{\mathrm{h}} 15^{\mathrm{m}}$ circa del giorno 14.

102. 6 dicembre

Un periodo sismico assai intenso e caratterizzato anche da una notevole frequenza di scosse si verificò nella zona dei Monti Volsini, a nord del Lago di Bolsena, dal 6 all'11 dicembre del 1957, colpendo violentemente numerosi centri dell'Alto Lazio e dell'estrema parte occidentale della provincia di Terni.

Nella faseia montuosa vuleanica che dal Monte Amiata, in Toscana, con andamento parallelo alla Catena Appenninica, si estende, attraverso i Monti Volsini, Cimini, Sabatini, Albani, fino alla Zona Flegrea in Campania, l'apparato dei Monti Volsini, la cui attività vulcanica si è spenta nel Quaternario, è uno dei maggiormente dotati di vivace sismicità. Attorno al Lago di Bolsena, formatosi in un complesso di eavità crateriche fusesi insieme, esistono infatti numerosi ed attivi focolari sismici i quali, di quando in quando, dànno origine a perturbazioni telluriche che, pur rimanendo generalmente circoscritte ad aree piuttosto ristrette, risultano talora di notevole intensità. Tali caratteristiche ebbe appunto il periodo sismico svoltosi nella prima metà del dicembre 1957 .

Esso ebbe inizio alle $5^{\mathrm{h}} 5^{\mathrm{m}}$ circa del 6 dicembre con una leggera scossa avvertita senza alcun allarme dalla popolazione della città di Orvieto e da quelle di numerosi paesi situati, come Castel Giorgio, Castel Viscardo, Acquapendente e Torre Alfina, fra le rive settentrionali del Lago di Bolsena e la valle del Fiume Paglia. Pochi minuti più tardi, alle $05^{\mathrm{h}} 55^{\mathrm{m}}$ circa, aecompagnata da forte rombo, sopravvenne la scossa principale del periodo sismico, la quale, nel paese di Castel Giorgio, 
raggiunse un'intensità quasi rovinosa, valutabile di VII-VIII grado della Scala Mercalli. Ingenti furono i danni causati dalla scossa in tale paese: lesioni generali e di notevole entità si ebbero in quasi tutti gli edifici e, in molti di essi, quelle prodotte alle strutture murarie portanti furono di tale gravità da renderli pericolanti ed inabitabili. La scossa fu fortissima

\section{[102] 6.Dicembre 1957}
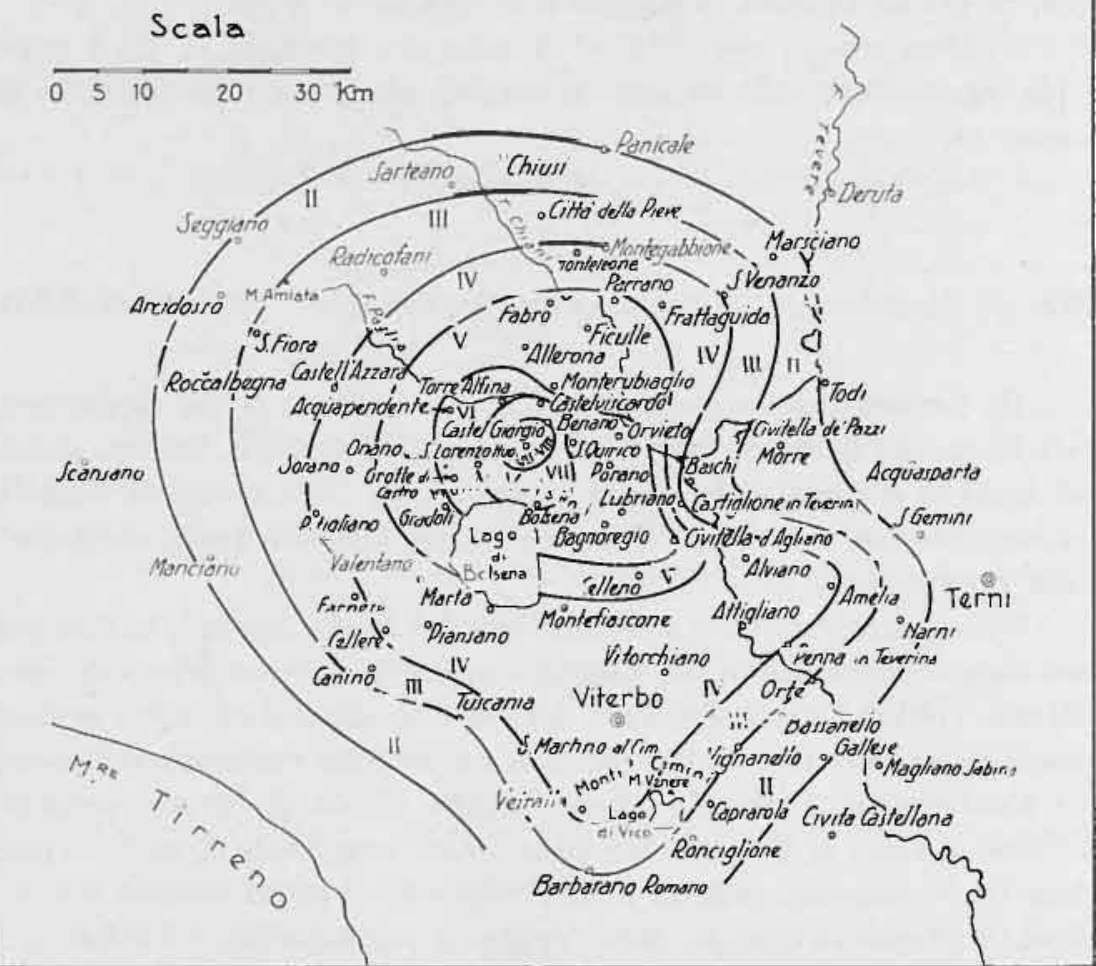

Fig. 64

(VII grado) a Castel Viscardo, a S. Lorenzo Nuovo, a Bolsena, a Viceno (fraz. di Castel Viscardo) e a Benano (frazione di Orvieto); in tutte queste località si verificarono crolli di comignoli e lesioni notevoli in numerosi edifici, sia pubblici che privati.

L'area isosismica in cui il movimento tellurico si manifestò con intensità molto forte (VI grado) si estese alla città di Orvieto; alle sue frazioni di Torre S. Severo, Sugano, Canonica, Rocca Ripesena; ai comuni 
di Grotte di Castro, Acquapendente, Porano, Lubriano, Bagnoregio e Civitella d'Agliano. In tali località, si ebbero fenditure, generalmente leggiere, in diversi fabbricati e, di una certa gravità, solo in qualche edificio di vechia ed irrazionale costruzione.

Tra i fenomeni più caratteristici concomitanti alla scossa, va segnalato, oltre al forte rombo udito in varie località, l'intorbidamento delle sorgenti nella zona di Castel Giorgio, Castel Viscardo e Sugano. Fu anche riferito che Ia sorgente del Tione, nei pressi di Sugano, interruppe per qualche tempo, immediatamente dopo la scossa, l'erogazione dell'acqua.

Eे interessante rilevare il rapidissimo decremento d'intensità verificatosi nel breve tratto, di appena due chilometri, che separa Castel Viscardo dalla sua frazione di Monterubiaglio. Il movimento sismico, che, come si è visto, investi Castel Viscardo con fortissima intensità, giunse infatti a Monterubiaglio con un'intensità non superiore al $\mathrm{V}$ grado.

Oltre che a Monterubiaglio, la scossa fu sentita di V grado nei comuni di Fabro, Parrano, Ficulle e Allerona, in provincia di Terni; di Onano, Gradoli e Celleno, in provincia di Viterbo. Di IV grado fu avvertita nella città di Viterbo ed in molti comuni della sua provincia, fra i quali Valentano, Marta, Cellere, Vitorchiano e Vetralla; nei comuni di Penna in Teverina, Amelia, Alviano, e S. Venanzo, della provincia di Terni; a Pitigliano e Castell'Azzara, in provincia di Grosseto.

Come appare dalla figura 64 , nelia quale sono tracciate le isosiste del movimento tellurico, l'estensione macrosismica, in relazione alla rilevante intensità nella zona epicentrale, fu alquanto limitata. Assai piccola dovette essere pertanto la profondità dell'ipocentro.

Dalle registrazioni ottenute negli Osservatori di Roma, Firenze e Bologna furono dedotti per le coordinate epicentrali i valori di $42^{\circ} 43^{\prime} \mathrm{N}$ e $12^{\circ} \mathrm{E}$, i quali individuano un punto situato tra Castel Giorgio e Castel Viscardo. L'ora della scossa all'epicentro fu $05^{\mathrm{h}} 54^{\mathrm{m}} 30^{\text {s }}$. Per la " magnitudo " del sisma fu calcolato il valore di 4,5 circa.

\subsection{Acquapendente - S. Lorenzo Nuovo (Viterbo)}

6 dicembre Castel Giorgio (Terni) Grado VI-VII

La violenta scossa di cui sopra succintamente si è detto venne seguita nella stessa giornata da ben 10 repliche succedutesi a brevi intervalli di tempo l'una dall'altra e che qui appresso elenchiamo indicando per 
ciascuna di esse Ia relativa intensità in gradi Mercalli raggiunta nella zona epicentrale:

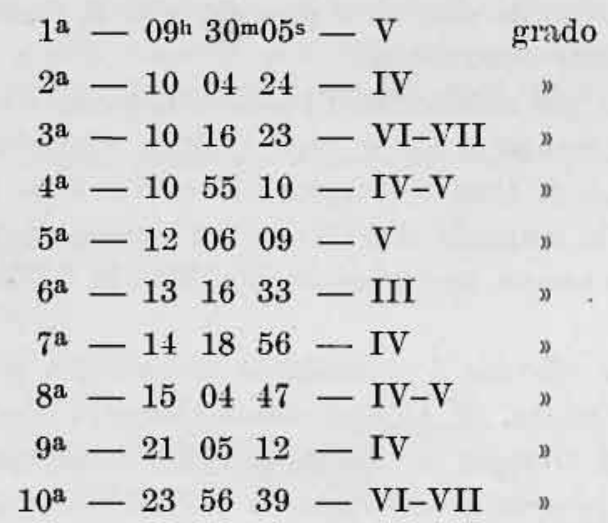

Tutte queste repliche furono registrate nell'Osservatorio sismico di Roma e le ore sopra indicate sono appunto quelle dedotte dalle registrazioni.

La notevole intensità della terza e della decima replica aggravò sensibilmente $\mathrm{i}$ danni prodotti dalla scossa principale. Le popolazioni di Castel Giorgio, Castel Viscardo e degli altri centri più colpiti abbandonarono le abitazioni in preda al più vivo sgomento, rifugiandosi nelle campagne in attendamenti sollecitamente apprestati dalle Aurorità civili e militari.

E possibile che le scosse sopra elencate non abbiamo avuto origine tutte da un identico centro sismico. Per la terza scossa ad esempio (quella delle $10^{\mathrm{h}} 16^{\mathrm{m}} 23^{\mathrm{s}}$ ), fu evidente uno spostamento verso ovest dell'epicentro. Essa risultò infatti di VI-VII grado nelle cittadine di Acquapendente e S. Lorenzo Nuovo, ove causò lesioni di un certo rilievo in numerose case, e d'intensità inferiore nelle vicine località, Castel Giorgio compresa. Nella zona sismica che si estende immediatamente a nord del Lago di Bolsena, numerosi e vicinissimi fra loro sono in realtà $i$ centri di scuotimento, sicchè non è improbabile che l'entrata in azione di uno di essi abbia determinato il risveglio di qualcuno degli altri.

La scossa fortissima delle $23^{\mathrm{h}} 56^{\mathrm{m}} 39^{\mathrm{s}}$ esplicò invece l'intensità sopra indicata di VI-VII grado ancora nel paese di Castel Giorgio e fu sentita meno fortemente nei paesi circostanti. La sua propagazione fu un po' più limitata di quella relativa alla scossa delle $00^{\mathrm{h}} 55^{\mathrm{m}}$. 
Il giorno 7 dicembre passò quasi completamente calmo nella zona del Lago Volsinio. Le scosse sismiche ricominciarono nelle prime ore del giorno 8 . Se ne verificarono cinque, leggiere, fra la mezzanotte del giorno 7 e le ore 6 del giorno 8; poi un'altra fortissima alle $17^{\mathrm{h}} 35^{\mathrm{m}} 53^{\mathrm{s}}$ pure del giorno 8. Anche questa raggiunse la sua maggiore intensità, valutata di VI-VII grado, nei pressi di Castel Giorgio ove si ebbero nuovi danni e sensibili aggravamenti nelle lesioni causate dalle scosse precedenti.

Dopo quest'ultima scossa, il periodo sismico andò nettamente diminuendo d'intensità: il giorno 9 si ebbero infatti tre scosse assai lievi avvertite quasi soltanto nel territorio di Castel Giorgio; cinque, il giorno 10 , anch'esse leggiere, eccetto la seconda, avvenuta alle $04^{\mathrm{h}} 57^{\mathrm{m}}$ cirea, che fu avvertita anche ad Orvieto e in numerosi comuni vicini a Castel Giorgio; quattro, lulle leggerissime, il giorno 11. Dopo l'ultima di queste, avvenuta alle $16^{\mathrm{h}} 06^{\mathrm{m}}$, i movimenti tellurici cessarono del tutto.

Qualche giorno dopo, e precisamente la mattina del 14 dicembre, Rocea Ripesena, piccola frazione distante circa 3 chilometri da Orvieto e costituita da un gruppo di case erette ai piedi di una rupe tufacea simile a quella orvietana ma di dimensioni di gran lunga più modeste, corse grave pericolo per il crollo di un enorme masso staccatosi dalla rupe ed infrantosi al suolo a brevissima distanza dall'abitato. Per quanto la rupe, profondamente incisa da solchi verticali ed indebolita da numerose gallerie scavate per l'estrazione di materiale, sia sottoposta ad una demolizione piuttosto rapida per opera degll agenti atmosferici, è da ritenere che gli scuotimenti sismici dei giorni precedenti abbiano agito su di essa accelerando il distacco e Ia frana del grossissimo masso.

Con il notevole periodo sismico dei Monti Volsini ebbe termine la serie dei più importanti movimenti tellurici verificatisi in Italia nel quinquennio 1953-1957. 


\section{A P P E N D I C E}

AI fine di rendere più facilmente consultabile la documentazione precedente, facciamo seguire l'elenco alfabetico delle località nelle quali, durante il periodo 1953-1957, furono avvertiti movimenti sismici d'intensità pari al $\mathrm{V}$ e superiore aI $\mathrm{V}$ della Scala Mercalli, indicando, a fianco di ciascuna località, i numeri dei paragrafi che ad essa si riferiscono.

\begin{tabular}{|c|c|c|}
\hline Località & Provincia & Paragrafi \\
\hline Acireale & Catania & 19 \\
\hline Acquapendente & Viterbo & 102 \\
\hline Airola & Benevento & 16 \\
\hline Alfero & Forli & 59,83 \\
\hline Alfonsine & Ravenna & 54 \\
\hline Allerona & Terni & $102,103,104$ \\
\hline Amandola & Ascoli Piceno & 71 \\
\hline Amatrice & Rieti & 81 \\
\hline Ampezzo & Belluno & 21 \\
\hline Ancona & - & 101 \\
\hline Andreis & Udine & $21,41,69$ \\
\hline Antrodoco & Rieti & 82 \\
\hline Arcidosso & Grosseto & 22 \\
\hline Argenta & Ferrara & 54 \\
\hline Argentera & Cuneo & 36 \\
\hline Arpaia & Benevento & 16 \\
\hline Arta & Udine & $5,69,75$ \\
\hline Asciano & Siena & 10 \\
\hline Ascoli Piceno & - & 28 \\
\hline Atella & Potenza & 86 \\
\hline Auronzo & Udine & 21 \\
\hline Avezzano & L'A quila & 11 \\
\hline Bagno di Romagna & Forli & $59,60,83,84$ \\
\hline Bagnoregio & Viterbo & 102 \\
\hline Balze & Forli & 59 \\
\hline Barete & L'Aquila & 73 \\
\hline Battaglia Terme & Padova & 54 \\
\hline Bellino & Cuneo & 36 \\
\hline Bernalda & Matera & 48 \\
\hline Bibbiena & Arezzo & 83 \\
\hline Bolano & La Spezia & 34 \\
\hline Bolognola & Macerata & 71 \\
\hline
\end{tabular}




\begin{tabular}{|c|c|c|}
\hline Località & Provincia & Paragrafi \\
\hline Bolsena & Viterbo & 102 \\
\hline Borgocoilefegato & Rieti & 82 \\
\hline Borgo S. Lorenzo & Firenze & 60 \\
\hline Bovolenta & Padova & 54 \\
\hline Brossasco & Cuneo & 36,37 \\
\hline Busca & Cuneo & 37 \\
\hline Gagli & Pesaro & 25 \\
\hline Cagnano Varano & Foggia & 30,33 \\
\hline Calciano & Matera & 48 \\
\hline Caldarola & Macerata & 71 \\
\hline Camaiore & Lueca & 47 \\
\hline Camerano & Ancona & 101 \\
\hline Campeggio & Bologna & 59 \\
\hline Campli & Teramo & 28 \\
\hline Camporeale & Trapani & 27 \\
\hline Cancellara & Potenza & 8,24 \\
\hline Canosa di Puglia & Bari & 6,67 \\
\hline Cantiano & Pesaro & 25 \\
\hline Capitello & Salerno & 38 \\
\hline Caramanico & Pescara & 68 \\
\hline Carpino & Foggia & 30,33 \\
\hline Carpinone & Campobasso & 70 \\
\hline Carrara & - & 47 \\
\hline Cascia & Perugia & 80,81 \\
\hline Castagno & Firenze & 60 \\
\hline Casteldelfino & Cuneo & 36,37 \\
\hline Castelfidardo & Ancona & 101 \\
\hline Castel Giorgio & Terni & $102,103,104$ \\
\hline Castelpetroso & Campobasso & 70 \\
\hline Castel San Niccolò & Arezzo & 83 \\
\hline Castel Viscardo & Terni & 102 \\
\hline Castrocaro & Forli & 60 \\
\hline Cavour & Torino & $36,37,61$ \\
\hline Celleno & Viterbo & 102 \\
\hline Cerignola & Foggia & 67 \\
\hline Cervasca & Cuneo & 36 \\
\hline Cervinara & Avellino & 16 \\
\hline Cesena & Forli & 15 \\
\hline Chiaramonte Gulfi & Ragusa & 78 \\
\hline Chiusdino & Siena & 14 \\
\hline Cittareale & Rieti & 64 \\
\hline Civezza & Imperia & 58 \\
\hline
\end{tabular}




\begin{tabular}{|c|c|c|}
\hline Località & Provincia & Paragrafi \\
\hline Civitella d'Agliano & Viterbo & 102 \\
\hline Civitella di Romagna & Forli & 60 \\
\hline Cleto & Cosenza & 1 \\
\hline Collestotte & Terni & 18 \\
\hline Colletorto & Campobasso & 13 \\
\hline Colloredo & Udine & 26 \\
\hline Colonna & Roma & 43 \\
\hline Comeglians & Udine & 69 \\
\hline Concerviano & Rieti & 82 \\
\hline Correggio & Reggio Emilia & 7 \\
\hline Cosenza & - & 51 \\
\hline Dovadala & Forlì & 20 \\
\hline Envie & Cuneo & 61 \\
\hline Fabro & Terni & 102 \\
\hline Ferentillo & Terni & 2 \\
\hline Ferrandina & Matera & 48 \\
\hline Fiamignano & Rieti & 82 \\
\hline Ficulle & Terni & 102 \\
\hline Filogaso & Catanzaro & 1 \\
\hline Firenzuola & Firenze & 59 \\
\hline Foggia & - & 67 \\
\hline Force & Ascoli Piceno & 89,94 \\
\hline Forlimpopoli & Forli & 15 \\
\hline Forni Avoltri & Udine & 69 \\
\hline Forno di Zoldo & Belluno & 21 \\
\hline Frosolone & Campobasso & 70 \\
\hline Galeata & Forli & $15,20,60,62,83$ \\
\hline Gatteo & Forlì & 15 \\
\hline Gemona del Friuli & Udine & 26 \\
\hline Giarratana & Ragusa & 78 \\
\hline Ginosa & Taranto & 48 \\
\hline Gradoli & Viterbo & 102 \\
\hline Grassano & Matera & 48 \\
\hline Grisì & Palermo & 27,49 \\
\hline Grizzana & Bologna & 59 \\
\hline Grotte di Castro & Viterbo & 102 \\
\hline Grottole & Matera & 48 \\
\hline Irsina & Matera & 48 \\
\hline
\end{tabular}




\begin{tabular}{|c|c|c|}
\hline Località & Provincia & Paragrafi \\
\hline Lagonegro & Potenza & 38 \\
\hline Lagopesole & Potenza & 86 \\
\hline La Spezia & - & 34 \\
\hline Lauria & Potenza & 86 \\
\hline Leni & Messina & 29 \\
\hline Leonessa & Rieti & 2 \\
\hline Ligosullo & Udine & 69 \\
\hline Lingua & Messina & 29 \\
\hline Loiano & Bologna & 59 \\
\hline Longiano & Forlì & 15 \\
\hline Lorenzago & Belluno & 21 \\
\hline Loreto & Ancona & 101 \\
\hline Lucera & Foggia & 67 \\
\hline Lubriano & Viterbo & 102 \\
\hline Maccliagodena & Campobasso & 70,72 \\
\hline Macra & Cuneo & 36 \\
\hline Malfa & Messina & 29 \\
\hline Mammola & Reggio Calabria & 92,93 \\
\hline Manfredonia & Foggia & 30,33 \\
\hline Maniago & Udine & 40 \\
\hline Marano sul Panaro & Modena & 98 \\
\hline Marcetelli & Rieti & 82 \\
\hline Maropati & Reggio Calabria & 1 \\
\hline Marmora & Cuneo & 36,37 \\
\hline Marradi & Firenze & 62 \\
\hline Marruci & L'Aquila & 73 \\
\hline Marzano Appio & Caserta & 45 \\
\hline Matera & - & 48 \\
\hline Medesano & Parma & 32 \\
\hline MeIdola & Forli & 15 \\
\hline Mercato Saraceno & Forli & 15,83 \\
\hline Messina & - & 1 \\
\hline Miglionico & Matera & 48 \\
\hline Modica & Ragusa & 78 \\
\hline Modigliana & Forli & 15 \\
\hline Moggio Udinese & Udine & 69 \\
\hline Monghidoro & Bologna & 59 \\
\hline Monteleone di Spoleto & Perugia & $2,80,81$ \\
\hline Montereggio & Piacenza & 23 \\
\hline Montese & Modena & 98 \\
\hline Monte Sant'Angelo & Foggia & $30,31,33,35,39,53,63,66,67$ \\
\hline Montescaglioso & Matera & 48 \\
\hline
\end{tabular}




\begin{tabular}{|c|c|c|}
\hline Località & Provincia & Paragrafi \\
\hline Naso & Messina & 87 \\
\hline Nicastro & Catanzaro & 1 \\
\hline Nicotera & Catanzaro & 1 \\
\hline Noceto & Parma & 32 \\
\hline Norcia & Perugia & 80 \\
\hline Numana & Ancona & 101 \\
\hline Onano & Viterbo & 102 \\
\hline Oncino & Cuneo & 61 \\
\hline Orvieto & Terni & 102 \\
\hline Ostana & Cuneo & 61 \\
\hline Palermo & - & 90 \\
\hline Paluzza & Udine & 69.75 \\
\hline Parrano & Terni & 102 \\
\hline Paularo & Udine & 69 \\
\hline Pedara & Catania & $29_{1}$ \\
\hline Perarolo & Belluno & 21 \\
\hline Peschici & Foggia & 67 \\
\hline Pescorocchiano & Rieti & 82 \\
\hline Petrella Salto & Rieti & 82 \\
\hline Piazza al Serchio & Lucea & 97 \\
\hline Pietramala & Firenze & 59 \\
\hline Pieve Santo Stefano & Arezzo & 85 \\
\hline Pinerolo & Torino & 36,37 \\
\hline Piscina & Torino & 37 \\
\hline Pisticei & Matera & 48 \\
\hline Pizzoli & L'Aquila & 73 \\
\hline Poggio Imperiale & Foggia & 67 \\
\hline Policastro & Selerno & 38 \\
\hline Pollara & Messina & 29 \\
\hline Pomarico & Matera & 48 \\
\hline Pontechianale & Cuneo & $36,37^{\circ}$ \\
\hline Porano & Terni & 102 \\
\hline Porto Recanati & Macerata & 101 \\
\hline Potenza & - & 24 \\
\hline Potenza Picena & Macerata & 101 \\
\hline Prato & Firenze & 44 \\
\hline Pratovecchio & Arezzo & 60 \\
\hline Prazzo & Cuneo & 36,37 \\
\hline Predappio & Forli & 15,83 \\
\hline Premileuore & Forli & 60,83 \\
\hline
\end{tabular}




\begin{tabular}{|c|c|c|}
\hline Località & Provincia & Paragrafi \\
\hline Radicondoli & Siena & 14 \\
\hline Ragusa & - & 78 \\
\hline Ramiseto & Reggio Emilia & 100 \\
\hline Raveo & Udine & 21,69 \\
\hline Resia & Udine & 69 \\
\hline Ricadi & Catanzaro & 1 \\
\hline Rignano Garganico & Foggia & 67 \\
\hline Rigolato & Udine & 69 \\
\hline Rinella & Messina & 29 \\
\hline Rionero nel Vulture & Potenza & 24 \\
\hline Roceabruna & Cuneo & 36 \\
\hline $\begin{array}{l}\text { Rocca San Casciano } \\
\text { Roma }\end{array}$ & Forli & $\begin{array}{c}15,20,60, \underset{2}{6}, 83,84 \\
2_{1}\end{array}$ \\
\hline Rovereto & Trento & 55 \\
\hline Salandra & Matera & 48 \\
\hline Salina & Messina & 29 \\
\hline Sambuca di Sicilia & Agrigento & 88 \\
\hline San Benedetto Val di Sambro & Bologna & 59 \\
\hline San Cosmo Albanese & Cosenza & 42 \\
\hline San Daniele & Udine & 26 \\
\hline San Demetrio Corone & Cosenza & 40,42 \\
\hline San Germano Chisone & Torino & 36,37 \\
\hline San Giorgio Albanese & Cosenza & 42 \\
\hline San Giovanni Rotondo & Foggia & $4,30,33,35,65,67,76,96$ \\
\hline San Godenzo & Firenze & 60 \\
\hline San Lorenzo Nuovo & Viterbo & 102 \\
\hline San Marco in Lamis & Foggia & $30,33,65,67$ \\
\hline San Martino di Finita & Cosenza & 50 \\
\hline Sannicandro Garganico & Foggia & 30 \\
\hline San Piero in Bagno & Forli & $59,60,62,83$ \\
\hline San Pietro Val Lemina & Torino & 61 \\
\hline Sanremo & Imperia & 79 \\
\hline San Severo & Foggia & 67 \\
\hline Santa Margherita Belice & Agrigento & 88 \\
\hline Santa Marina Salina & Messina & 29 \\
\hline Sant'Anatolia di Nareo & Perugia & 95 \\
\hline Sant'Angelo in Grotte & Campobasso & 70,72 \\
\hline Santa Sofia & Forli & $15,60,62,83,84,91$ \\
\hline Sant'Elena Sannita & Campobasso & 70 \\
\hline Sant'Ilario & Potenza & 86 \\
\hline Sant'Onofrio & Catanzaro & 1 \\
\hline Santo Stefano di Cadore & Belluno & 69 \\
\hline
\end{tabular}




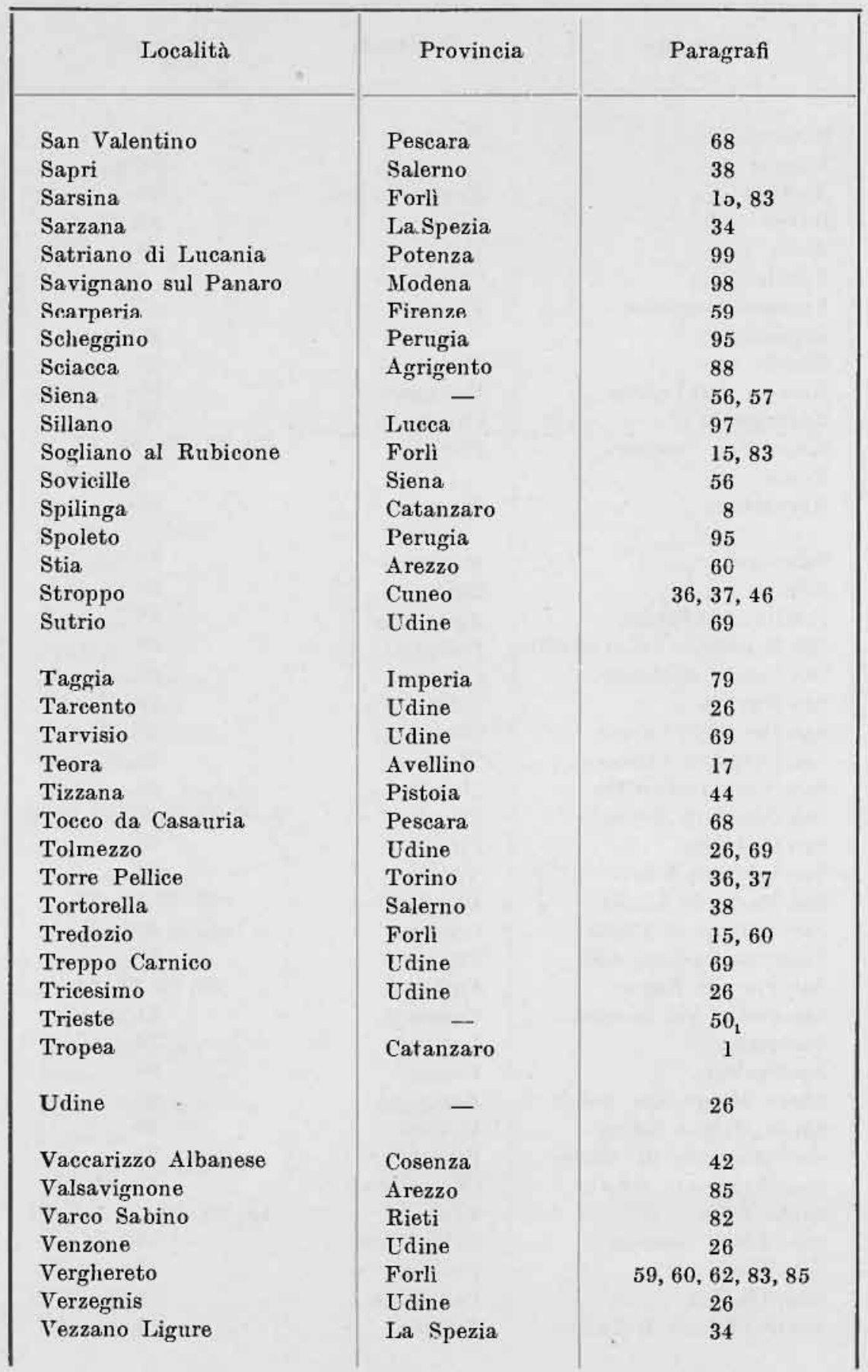




\begin{tabular}{|l|l|c|}
\hline \multicolumn{1}{|c|}{ Località } & Provincia & Paragrafi \\
\cline { 2 - 3 } Vibonati & & \\
Vibo Valentia & Salerno & 38 \\
Vieste & Catanzaro & 1 \\
Vignola & Foggia & 67 \\
Villafranca Piemonte & Modena & 98 \\
Vito d'Asio & Torino & 37 \\
Vittorio Veneto & Udine & 26 \\
Vizzini & Treviso & 21 \\
& Catania & 3 \\
Zafferana Etnea & & 52,77 \\
Zocca & Catania & 98 \\
Zungri & Modena & 1 \\
\hline
\end{tabular}

\section{RIASSUNTO}

Il presente lavoro è uno studio macrosismico dei terremoti avvenuti in Italia dal 1953 al 1957 e che nella zona epicentrale hanno raggiunto intensità pari e superiori al $V$ grado 'della Scala Mercalli. L'Autore si è servito delle notizie macrosismiche pervenute all'Ufficio Centrale di Meteorologia ed Ecologia Agraria e di quelle da lui stesso raccolte direttamente, in alcuni casi anche mediante sopraluoghi, dalle localita dove $i$ movimenti sismici si sono verificati.

La descrizione macrosismica di ciascun terremoto, eccetto in quei casi in cui le notizie a disposizione erano insufficienti, è stata completata con il disegno delle relative isosiste. Accompagna il testo una carta generale degli epicentri che dà una visione sintetica dell'attività sismica in Italia durante il periodo esaminato.

Le ore indicate nel testo sono espresse nel "Tempo medio dell'Europa centrale ".

\section{ABSTRACT}

The present work is a macroseismic study of earthquakes which took place in Italy from 1953 to $195 \%$ and which, in the epicentral area, have reached intensity equal or superior to $V$ degree on Mercalli Scale. In its compilation, the Author has utilized macroseismic data obtained from the 
"Ufficio Centrale di Meteorologia e di Ecologia Agraria" and the data collected by himself personally and sometimes during a study on the spot at places where seismic movements took place.

The macroseismic description of each earthquake, except in cases when the available data were not adequate, has been completed with a drawing of pertaining isoseistes. The report is supplemented with a general map of epicentres which gives a succint vision of seismic activity recorded in Italy during the period studied.

The hours indicated in the report are given in "Mean CentralEuropean Time".

\section{B I B L I O G R A F I A}

( $\left.{ }^{1}\right)$ M. Baratta, I terremoti d'Italia, 333, Fr. Bocca, Torino, 1901.

$\left({ }^{2}\right)$ D. Di Finippo e L. Marcelli, Uno studio sul terremoto di Cefalonia del 12 agosto 195.3, con particolare riguardo alla natura fisica della scossa all'ipocentro, "Annali di Geofisica", VII, 547, (1954).

( ${ }^{3}$ ) A. Cavasino, I Terremoti d'Italia nel trentacinquennio 1899-1933, 23, Ist. Poligr. dello Stato, 1935.

(4) S. Cocuzza Suvestri, Sul periodo sismico di Pedara (Etna) manifestatosi nei primi mesi del 1955, "Annali di Geofisica ", IX, 557, (1956).

$\left({ }^{5}\right)$ E. Peterscinmt, Quelques données nouvelles sur les séismes profonds de la Mer Tyrrhénienne, "Annali di Geofisica n, IX, 305, (1956).

$\left({ }^{6}\right)$ A. GrRlanda, Sul terremoto profondo delle Isole Eolie del 23 novembre 1954, "Annali di Geofisica ", VIII, 437 (1955).

( ${ }^{2}$ P. Calor, Attività sismica in Italia nel decennio 1930.1939, 43, Felice Le Monnier, Firenze. 


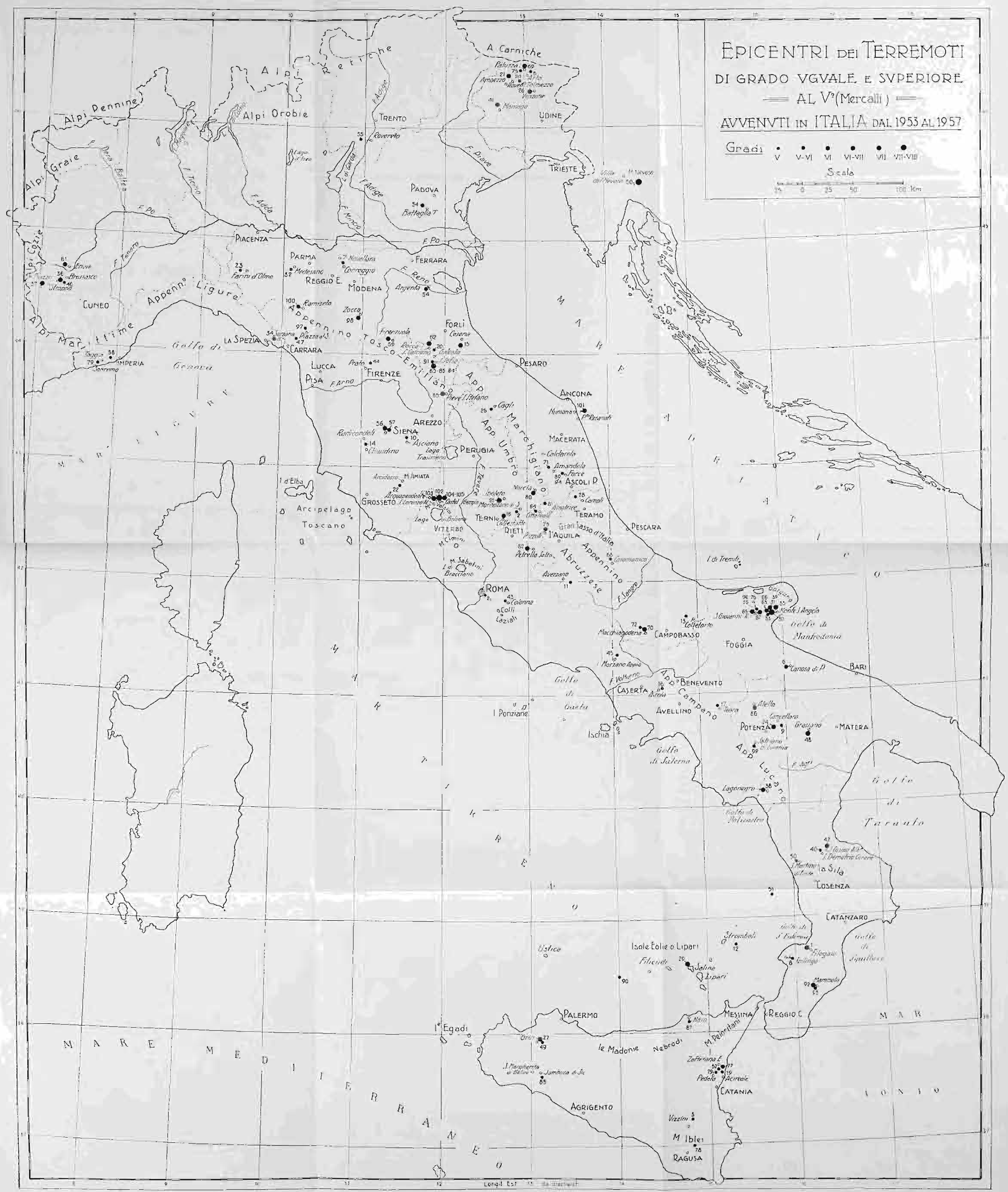

